\title{
A Search for Doubly-Charged Higgs Bosons at the Tevatron
}

\author{
By \\ SASHA BAROIANT \\ B.S. (University of California, Davis) 1999 \\ DISSERTATION
}

Submitted in partial satisfaction of the requirements for the degree of DOCTOR OF PHILOSOPHY

in

Physics

in the

OFFICE OF GRADUATE STUDIES

of the

UNIVERSITY OF CALIFORNIA

DAVIS

Approved:

Committee in Charge

2006 
Sasha Baroiant

December 2006

Physics

\title{
A Search for Doubly-Charged Higgs Bosons at the Tevatron
}

\begin{abstract}
We search for the pair production of doubly charged Higgs particles followed by the lepton-flavor violating decay of each Higgs into electron-and-tau and muonand-tau pairs using $350 \mathrm{pb}^{-1}$ of data collected by the CDF II experiment at the Fermilab Tevatron. Separate searches investigate cases where three or four finalstate leptons are detected, and the limits for each exclusive decay mode reflect the combined results of both searches. Assuming the $H_{L}^{ \pm \pm}$decays exclusively into likesign electron-and-tau pairs, we set a lower limit on its mass of $114 \mathrm{GeV} / c^{2}$ at the $95 \%$ confidence level. In the case of exclusive muon-and-tau decays, we set a lower mass limit of $112 \mathrm{GeV} / c^{2}$ also at the $95 \%$ confidence level.
\end{abstract}


Tiger, tiger burning bright

In the forrest of the night,

What immortal hand or eye

Could Frame thy fearful symmetry?

-William Blake 


\section{Acknowledgements}

This thesis is dedicated to my parents, Ania Balaian and Henry Baroiant.

I would like to thank my advisor, Professor Richard Lander, for introducing me to high energy physics. I was pretty much hooked after he taught me Gauge Theory, and my interest in experimental physics developed because he gave me the opportunity to work on the CDFII upgrade. As a graduate student, I very much appreciated his continual encouragement and his contagious excitement about physics.

The UCD HEP group has been instrumental in my development as a physicist. The concepts learned in Professor Dave Pellett's electronics classes helped me to understand and debug ISL ladders and DAQ equipment. Professor Max Chertok's experimental methods class greatly facilitated data analysis at CDF, and I thank him also for introducing me to tau physics. I always looked forward to Professor Phil Yager's classes - his lectures often came together in a way that gave me goosebumps. I very much enjoyed many discussions with Dr. John Smith regarding physics, and beyond. Along with Professors Conway, Erbacher, and Tripathi, these people make the 3rd floor of the Physics Building an exciting and nurturing environment for UCD graduate students.

I am very happy to have worked closely with Professor Alexei Safonov of Texas A\&M University. His insight and advice were indispensable to this work, and I feel fortunate to have assimilated even a small fraction of his methods. The members of the CDF Exotics group, especially Chris Hays, Beate Heinemann, Teruki Kamon, Amit Lath, and Stephan Lammel, have made many helpful suggestions regarding my analysis. All of us at CDF are much obliged to Dr. Aron Soha for his important contribution as Operations Manager. I'm also indebted to him for his hospitality at Chez Soha.

I am very thankful for the hospitality and friendship of my fellow student Stanley 
Forrester, and his family. The friendship of Dr. Juan Pablo Fernandez and his family was one of my most significant discoveries at FNAL. Along with Giobatta Lanfranco, who would not hesitate to ride up and down "garbage mountain" with me, they have made my stays at the lab very enjoyable, and have kept me from getting overly homesick. I'm especially thankful for the friendship of Marc Bavois, who kindly distills and distributes beauty in all forms to a busy, distracted, graduate student.

I'm most grateful to the Balaian and Baroiant families for their love, support, and encouragement in all regards. I can't thank my parents enough, who have managed to provide me with such security, comfort, and opportunities. I'm especially blessed to have such wonderful siblings, and they have contributed to my happiness throughout. I am continually amazed by, and thankful for, my wife, Megan - a constant source of joy, beauty, and inspiration. 


\section{Contents}

1 Theoretical Overview 1

1.1 Introduction . . . . . . . . . . . . . . . . . . 1

1.2 An Example: Quantum Electrodynamics as a Gauge Theory . . . . 3

1.3 Standard Model . . . . . . . . . . . . . . . . . . . 6

1.3.1 Historical Development . . . . . . . . . . . . 6

1.3.2 Matter Fields . . . . . . . . . . . . . . . . . 8

1.3.3 Gauge Bosons . . . . . . . . . . . . . . . . . . . 9

1.3.4 Electroweak Unification . . . . . . . . . . . . . . . . 9

1.3.5 The Unification of the Strong and Electroweak Interactions . 10

1.3.6 The Higgs Mechanism . . . . . . . . . . . . . . . . . . . 10

1.3.7 Remarks on the SM . . . . . . . . . . . . . . 13

1.4 The Left-Right Symmetric Model . . . . . . . . . . . . . . . . . . . . 17

1.5 Higgs Triplet Model . . . . . . . . . . . . . . . . . . . . . . 18

2 Experimental Apparatus $\quad 20$

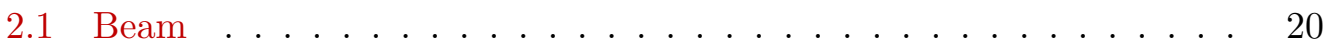

2.2 Particle Accelerators . . . . . . . . . . . . . . . . . . 21

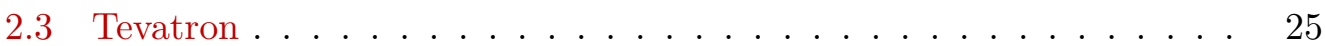

2.3 .1 Protons . . . . . . . . . . . . . . . . 25

2.3.2 Antiprotons . . . . . . . . . . . . . . . . 28

2.3.3 Tevatron Performance . . . . . . . . . . . . . . . 30 
2.4 CDF Detector . . . . . . . . . . . . . . . . . . 31

2.4.1 The Silicon Tracking Detectors . . . . . . . . . . . . . 33

2.4.2 The Central Outer Tracker . . . . . . . . . . . . . 38

2.4.3 The Time Of Flight Detector . . . . . . . . . . . . . 41

2.4 .4 The Calorimeters . . . . . . . . . . . . . . . . . . 43

2.4.5 The Muon System . . . . . . . . . . . . . . . . 48

2.4.6 The Luminosity Monitor . . . . . . . . . . . . . . . . . . 51

2.4 .7 Triggers and DAQ . . . . . . . . . . . . . 52

2.5 Software Tools . . . . . . . . . . . . . . . 56

$\begin{array}{lll}3 & \text { Search Signature } & 59\end{array}$

$3.1 \quad H^{ \pm \pm}$Production . . . . . . . . . . . . . . . . . . . 59

$3.2 H^{ \pm \pm}$Decay . . . . . . . . . . . . . . . . . 61

3.3 Search Signature . . . . . . . . . . . . . . . . 62

$\begin{array}{lll}4 & \text { Event Selection } & 67\end{array}$

4.1 Lepton Reconstruction . . . . . . . . . . . . . . . . 67

4.1 .1 Reconstruction of Electrons . . . . . . . . . . . . . 68

4.1 .2 Reconstruction of Muons . . . . . . . . . . . . . 70

4.1.3 Reconstruction of Tau Leptons . . . . . . . . . . . . . . . 72

4.2 Lepton Identification . . . . . . . . . . . . . . . . . . . 80

4.2 .1 Electron Identification . . . . . . . . . . . . . . . . 82

4.2 .2 Muon Identification . . . . . . . . . . . . . . . 87

4.2.3 Hadronic Tau Identification . . . . . . . . . . . . . . . . . 90

4.2.4 Additional Lepton Types . . . . . . . . . . . . . . . . . . 94

4.3 Missing Transverse Energy and Related Corrections _ . . . . . . . . 97

4.4 Datasets . . . . . . . . . . . . . . . . . . . . 98

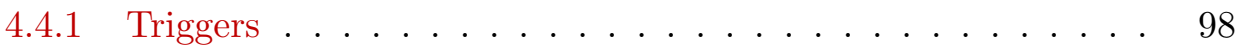

4.4 .2 Good Run Lists and Datasets . . . . . . . . . . . . . . 103

4.4.3 Three and Four Lepton Samples . . . . . . . . . . . . 106 
4.5 SM Backgrounds after Lepton ID . . . . . . . . . . . . . . 106

4.6 Event-Level Selections . . . . . . . . . . . . . . . . . . . . 110

4.6.1 Event Selections for Three-Lepton Events . . . . . . . . . . . 111

4.6.2 Event-Level Selections for Four-Lepton Events . . . . . . . 115

$\begin{array}{llr}5 & \text { Background Determination } & 118\end{array}$

5.1 Monte Carlo Samples . . . . . . . . . . . . . . . . . . . . . . . . . . 118

5.2 Blind Analysis . . . . . . . . . . . . . . . . . . . . . . 119

5.3 Backgrounds and Control Regions for the er Search . . . . . . . . 120

5.3.1 QCD and Photon+jets Backgrounds .......... 120

5.3.2 W+jets Background and Control Region . . . . . . . . . . 125

5.3.3 Z $\rightarrow$ ee Background and Control Region . . . . . . . . . . . 127

5.3.4 Diboson and $t \bar{t}$ Backgrounds . . . . . . . . . . . . . . . 129

5.3.5 Additional Control Regions . . . . . . . . . . . . . . . . . 130

5.3 .6 Low $H_{T} \ldots \ldots \ldots \ldots \ldots \ldots \ldots$

5.3.7 Control Regions for the 4-Track Region . . . . . . . . . . . 131

5.4 Backgrounds and Control Regions for the $\mu \tau$ Search . . . . . . . . . 132

5.4 .1 Dilepton Control Region . . . . . . . . . . . . . . . . 133

5.4 .2 Loose Selections . . . . . . . . . . . . . . . . . 135

5.4 .3 Low $H_{T} \ldots \ldots \ldots \ldots \ldots \ldots$

5.4 .4 Reverse Tau TIso $\left(\right.$ high $\left.H_{T}\right) \quad \ldots \ldots \ldots$. . . . . . 137

5.4.5 Mismeasured Track $p_{T}$ Study with $Z \rightarrow$ ee Data . . . . . 138

5.5 Systematic Errors on Background Acceptance . . . . . . . . . . . . 139

6 Signal Acceptance 141

6.1 Expected Signal . . . . . . . . . . . . . . . . . . . . . 141

6.2 Systematic Uncertainties on Signal Acceptance . . . . . . . . . . . 142

6.2 .1 Lepton Identification . . . . . . . . . . . . . . . . . . . . 143

6.2 .2 Track Isolation . . . . . . . . . . . . . . . . . . . . 143

$6.2 .3 \pi^{0}$ Isolation . . . . . . . . . . . . . . . . . . . . . . . . . 144 
6.2.4 Calorimeter Energy Scale . . . . . . . . . . . . . . . 145

6.2.5 Parton Distribution Functions . . . . . . . . . . . . . . 145

6.2 .6 Initial State Radiation . . . . . . . . . . . . . . . . 146

6.2 .7 Trigger Efficiency . . . . . . . . . . . . . . . 147

6.3 Systematic Uncertainties on Signal Production . . . . . . . . . . 147

6.3.1 Uncertainty on Theoretical Cross Section. . . . . . . . . . 147

6.3 .2 Luminosity . . . . . . . . . . . . . . . . . . . . 147

6.3.3 Parton Distribution Functions . . . . . . . . . . . . 147

7 Limit Setting Procedure $\quad 149$

7.1 Expected and Observed Cross section Limits . . . . . . . . . . . . 152

7.2 Summary . . . . . . . . . . . . . . . . . . 152

$\begin{array}{lr}\text { A The Goldstone Theorem } & 154\end{array}$

B Massive Neutrinos 156

$\begin{array}{ll}\text { C Seesaw Mechanism } & 158\end{array}$

$\begin{array}{lr}\text { D Tau Energy Correction } & 160\end{array}$

$\begin{array}{ll}\text { E Conversion Tagging Efficiency } & 164\end{array}$

F Interesting Event in Low $M_{L S}$ Region $\quad 166$

$\begin{array}{lr}\text { Bibliography } & \mathbf{1 7 0}\end{array}$ 


\section{Chapter 1}

\section{Theoretical Overview}

The Standard Model (SM) of particles and fields has shown impressive predictive power and remarkable agreement with precise experimental measurements. Despite this, it is widely hoped that the SM will be replaced by, or incorporated into, a unified explanation of all phenomena. Recently, direct evidence for physics contrary to the $\mathrm{SM}^{1}$ has come from neutrino experiments: the neutrinos, assumed to be identically massless in the SM, are now proven to be massive. Some extensions of the SM have been predicting massive neutrinos for decades. Most of these theories contain complex scalar triplet fields that impart masses to the neutrinos. This thesis describes a search for doubly charged Higgs bosons $\left(H^{ \pm \pm}\right)$, which are prominent members of these scalar triplets. In this section we highlight some aspects of the experimental and theoretical foundation of the SM and briefly discuss two models that predict massive neutrinos and doubly charged Higgs bosons.

\subsection{Introduction}

The goal of particle physics is to describe the fundamental constituents of matter and their interactions. Physicists aim to develop a coherent mathematical formalism that explains all known phenomena. The desire for a unified theory is well-motivated by

\footnotetext{
${ }^{1}$ That is not to say beyond the scope of the SM, the gravitational interaction is an obvious example of physics beyond the scope of the SM.
} 
historical successes. For example, one of the most celebrated unifications in physics is embodied in Maxwell's equations. Two forces are shown to be related - a changing electric field can produce an electric field, and vice versa. There is almost perfect symmetry in the equations that describe the electric and magnetic fields ${ }^{2}$. The decoupling of Maxwell's first order partial differential equations led to wave equations for the electromagnetic field and corresponding solutions that had a propagation velocity and polarization properties consistent with experimental data regarding light. Maxwell wrote "We can scarcely avoid the inference that light consists in the transverse undulations of the same medium which is the cause of electric and magnetic phenomena." [1]. The unified description of electromagnetism led to new understanding of nature of light, but perhaps more importantly, Maxwell's description cast into doubt the validity of Galilean relativity and led to the development of special relativity ${ }^{3}$, which itself is a unification of three dimensional space and time. The Lorentz transformations associated with special relativity have the property that they preserve the generalized distance $d x^{2}$ in Minkowski space:

$$
d x^{2} \equiv \sum_{i=1}^{3}\left(x_{i}^{2}\right)-c^{2} t^{2}=\sum_{i=1}^{3}\left(x_{i}^{\prime 2}\right)-c^{2} t^{\prime 2}=d x^{\prime 2}
$$

To comply with special relativity, a theory's observables must be invariant under the Lorentz transformations. Such theories are said to be Lorentz invariant or to possess Lorentz symmetry.

As a guiding principal, symmetry has proved to be of paramount importance to the advancement of physics. Noether's theorem[2] relates symmetries of the Lagrangian to conserved quantities. Conservation of linear momentum holds for any theory that is invariant under spacial translations, while invariance under temporal translations result in the conservation of energy. If a Lagrangian possesses rotational symmetry, then the resulting dynamics will conserve angular momentum. A

\footnotetext{
${ }^{2}$ Although the apparent lack of a magnetic charge can be as glaring as the symmetry itself!

${ }^{3}$ Special relativity, in turn, enabled the interpretation of magnetism as a relativistic effect of electrodynamics!
} 
second class of transformations can be carried out in an "internal" space. These are called "phase" or "gauge" transformations. An observable of the form $|\phi|^{2}=\phi^{*} \phi$, is manifestly invariant under a transformation $\phi \rightarrow e^{i \alpha Q} \phi$, where for convenience we factor $\alpha$ out of $Q$ so that later, any spacial dependence of the transformations can be absorbed in $\alpha(x)$. The conserved quantity in such cases is a "generalized" charge, Q, which is to be interpreted as some intrinsic trait of a particle or field such as electric charge - that does not depend on its space-time coordinates ${ }^{4}$. The unitary transformations $e^{i \alpha Q}$ can be thought of as generalized rotations in internal spaces. The operators $Q$ are generators of transformations for Lie groups. The example above corresponded to the $U(1)$ group. So far, we have only discussed global transformations: the transformation is the same regardless of space-time location. One can also define local gauge transformations, where the change $\delta \phi$ of the field depends on the space-time coordinates: $\phi \rightarrow \phi=e^{i \alpha(x) Q} \phi$. The principle of $l o-$ cal gauge invariance has proven to be indispensable to the description of physical interactions at the most fundamental level. We offer one such example below.

\subsection{An Example: Quantum Electrodynamics as a Gauge Theory}

Consider the Dirac Lagrangian ${ }^{5}$, which is consistent both with special relativity and quantum mechanics, and describes a free, spin $1 / 2$ particle of mass $\mathrm{m}$.

$$
\mathcal{L}_{0}=\bar{\psi}(x)(i \not \partial-m) \psi(x)
$$

Requiring (1.2) to be invariant under the global $U(1)$ transformation

$$
\psi(x) \rightarrow \psi^{\prime}(x)=e^{-i q \alpha} \psi(x)
$$

\footnotetext{
${ }^{4}$ The invariance of particle's electric charge with respect to Lorentz transformations can be demonstrated to $10^{-10}$ accuracy just by measuring the electrical neutrality of bulk matter at different temperatures[3]!

${ }^{5}$ This discussion assumes that the reader has some familiarity in the Dirac equation and related mathematical structures. References can be found in the usual textbooks[4, 5, 6, 7].
} 
leads to a conserved current and the interpretation of $q$ as the electric charge associated with the field $\psi$. Let's see what happens when one requires (1.2) to be invariant under a local phase transformation:

$$
\psi(x) \rightarrow \psi^{\prime}(x)=e^{-i q \alpha(x)} \psi(x)
$$

The Lagrangian is not invariant to the local transformation because the derivatives of the fields do not transform as the fields do:

$$
\mathcal{L}_{\prime}=\bar{\psi}(x)\left(i \gamma^{\mu} \partial_{\mu}-m\right) \psi(x) \rightarrow \mathcal{L}_{0}^{\prime}=\bar{\psi}^{\prime}(x)\left(i \gamma^{\mu}\left(\partial_{\mu}-i q \partial_{\mu} \alpha(x)\right)-m\right) \psi^{\prime}(x) .
$$

The offending term can be canceled if we replace the derivative $\partial_{\mu} \psi$ with what is called the covariant derivative $D_{\mu} \psi$ :

$$
D_{\mu} \psi \equiv\left(\partial_{\mu}+i q A_{\mu}(x)\right) \psi
$$

The transformed "gauge" field $A_{\mu}^{\prime}(x)$ must cancel the term $i q \partial_{\mu} \alpha(x)$, so we need $A_{\mu}(x)$ to transform as:

$$
A_{\mu}(x) \rightarrow A_{\mu}^{\prime}(x)=A_{\mu}(x)+\partial_{\mu} \alpha(x)
$$

After replacing $\partial_{\mu}$ with $D_{\mu}$, the Lagrangian is invariant under the local $U(1)$ transformation:

$$
\begin{array}{r}
\mathcal{L}=\bar{\psi}(x)\left(i \gamma^{\mu}\left(\partial_{\mu}+i q A_{\mu}(x)\right)-m\right) \psi(x) \\
=\bar{\psi}(x)\left(i \partial_{\mu}-m\right) \psi(x)+q \bar{\psi}(x) \gamma^{\mu} A_{\mu}(x) \psi(x) \equiv \mathcal{L}_{0}+\mathcal{L}_{I}
\end{array}
$$

The Lagrangian now has a new term $\mathcal{L}_{\mathcal{I}}$ that couples the $A_{\mu}(x)$ to the fermion fields with an interaction strength proportional to the electric charge $q$. We can interpret the gauge field $A_{\mu}(x)$ as the photon. Covariance of $\partial_{\mu} \psi$ under a local $U(1)$ transformation requires the photon field! Note that a photon mass term $A_{\mu} A^{\mu}$ 
would again destroy the Lagrangian's covariance, but a kinetic term can and should be added, since we interpret $A_{\mu}(x)$ as a genuine field in its own right. The simplest ${ }^{6}$ gauge-invariant term is of the form:

$$
\left(\partial_{\mu} A_{\nu}-\partial_{\nu} A_{\mu}\right)\left(\partial^{\mu} A^{\nu}-\partial^{\nu} A^{\mu}\right) \equiv F_{\mu \nu} F^{\mu \nu}
$$

which we recognize to be the field strength tensor from electrodynamics. Thus we can write our covariant Lagrangian.

$$
\mathcal{L}=\bar{\psi} i \gamma^{\mu}\left(\partial_{\mu}+i q A_{\mu}\right) \psi-m \bar{\psi} \psi-\frac{1}{4} F_{\mu \nu} F^{\mu \nu}
$$

The above Lagrangian describes quantum electrodynamics - one of the most successful physical theories to date.

It is remarkable that the simple requirement of local $U(1)$ gauge invariance for the Dirac Lagrangian results in the necessary creation of the photon field. The local gauge symmetry requirement led to the unification of quantum mechanics and electromagnetism. Although QED was initially very well received, if only for its sheer elegance, it was soon discovered that the theory included many divergent integrals. One way to deal with these infinities was to use a scheme called "renormalization". The divergent integrals were absorbed into the masses and coupling constants of the theory, which were scaled from the infinite to the finite ${ }^{7}$. The renormalization was possible only because QED is an unbroken gauge symmetry. It is easy to see why the local gauge invariance principal is attractive to the model builder.

\footnotetext{
${ }^{6}$ One must, in general, add all possible gauge invariant terms, unless there's a good reason not to. In this case, the term $\epsilon^{\mu \nu \rho \sigma} F_{\mu \nu} F^{\rho \sigma}$ is omitted because it contributes only as a surface term to the action, and is of no consequence to the equations of motion[6]. The term $\bar{\psi} \sigma_{\mu \nu} \psi F^{\mu \nu}$ is also gauge invariant, but is omitted because it would generate divergent integrals which cannot be made finite by any known scheme.

${ }^{7}$ This may sound silly, but consider the effect of charge screening in dielectric media, for example, or, the minimization of the action to get the Euler-Lagrange equations of motion: any constant term in the action is inconsequential, even if it is infinite.
} 


\begin{tabular}{cccc}
\hline Force & Mediator & Coupling Constant & Range \\
\hline EM & $\gamma$ & $1 / 137$ & $\infty$ \\
Strong & $\mathrm{g}$ & 1 & $10^{-15} \mathrm{~m}$ \\
Weak & $W^{ \pm}, Z$ & $10^{-6}$ & $10^{-18} \mathrm{~m}$ \\
Gravity & graviton & $10^{-40}$ & $\infty$ \\
\hline
\end{tabular}

Table 1.1: The four known forces and their mediators.

\subsection{Standard Model}

Our current understanding is that four fundamental forces, or interactions, are responsible for all phenomena. Gravity and electromagnetism are the forces which we constantly encounter in our daily lives. The other two forces less commonly experienced or perceived, partially because their effective ranges are shorter than $10^{-13}$ meters. The strong force binds together the nucleons in an atom's nucleus, and the weak force is responsible for neutron decay. The four interactions and their mediators are summarized in Table1.1.

The Standard Model describes the strong, weak, and electromagnetic interactions in the framework of a gauge theory. The model is described fully in $[5,7,6,8]$. We give only a brief overview here, and focus mainly on the electroweak sector, as this thesis describes an experimental search for evidence of electroweak sectors beyond what is predicted by the SM. We start with a brief historical overview of developments in particle physics in order to motivate the structure of the SM.

\subsubsection{Historical Development}

By the mid 1930's, quantum mechanics was on firm footing and QED was in development. Dirac had already discovered the relativistic wave equation for electrons and its negative-energy solutions had led him to predict anti-particles, which were discovered in 1932. In the same year, Chadwick discovered the neutron, and it was quickly accepted that protons and neutrons comprise atomic nuclei. Yukawa hypothesized that interactions between nucleons are mediated by three bosons, and he related the effective range of the interactions to the mediators' masses, which he 
estimated to be $\mathcal{O}\left(100 \mathrm{MeV} / c^{2}\right)^{8}$. The $\beta$ decay of nuclei was still somewhat of a mystery: the momentum spectrum of the emitted electrons was continuous whereas it should have been sharply peaked to reflect the definite energy difference between the neutron and proton $(n$ and $p$ ). In 1934, Pauli suggested that $\beta$ decay was the reaction $n \rightarrow p+e^{-}+\bar{\nu}_{e}$. The lack of direct evidence for the particle suggested that it rarely interacted with common matter. That same year, Fermi had an effective theory for the "weak" interaction:

$$
\mathcal{L}_{\text {weak }}=\frac{G_{F}}{\sqrt{2}}\left(\bar{\psi}_{p} \gamma_{\mu} \psi_{n}\right)\left(\bar{\psi}_{e} \gamma^{\mu} \psi_{\nu}\right)
$$

This "4-point" interaction worked well at low energies, but suffered from unitarity breakdown at high energies.

Stevenson and Street discovered the muon by using a cloud chamber to investigate cosmic rays[9], and at first it was thought to belong to the family of mediators proposed by Yukawa. Soon it was clear that the muon did not interact strongly enough to be associated with the "strong" interactions between nucleons, and so the muon was classified as a "second generation" lepton", since it was identical to the electron, but 200 times heavier. The list of weak interactions grew to include muon-decay $\mu^{-} \rightarrow e^{-}+\bar{\nu}_{e}+\nu_{\mu}$ and muon capture: $\mu^{-}+p \rightarrow \nu_{\mu}+n$. Lack of direct experimental evidence for the neutrinos was not so troubling as indirect evidence abounded. However, weak decays of some newly discovered particles stared to show mysterious behavior. Two particles, the $\theta^{+}$and $\tau^{+}$, had the exact same characteristics, except that they decayed into states of opposite parity. Their relatively long lifetimes indicated that these particles underwent weak decays. One had to assume that either there are two particles that are identical in every respect except for their decays, or that two different decay modes of the same particle were observed. The latter view implied that the weak interaction violated parity. Yang

\footnotetext{
${ }^{8} \mathrm{~A}$ massive mediator would result in the potential $\Phi(x)=q \frac{e^{-\mu r}}{r}[3]$ with $\mu=m c / \hbar$.

${ }^{9}$ The word lepton derives from Greek "leptos" meaning "slender" or "delicate". Leptons do not participate in the strong interaction. Particles that partake in the strong interaction are called "hadrons", from Greek "hadros" meaning "burly".
} 
and Lee[10] suggested that this might be the case, and shortly thereafter $\mathrm{Wu}[11]$ demonstrated experimentally that the weak interaction violated parity - it only involved the left-handed ${ }^{10}$ components of the Dirac fields. Also, direct experimental evidence[12] showed that all weakly interacting neutrinos were left-handed ${ }^{11}$.

Yukawa's theory of the meson-mediated strong force enjoyed a resurgence after the discovery $\pi^{ \pm}$and $\pi^{0}$ mesons. In 1957, Schwinger [13], Lee, and Yang[14] developed the idea of "intermediate vector bosons" (IVB) that mediated the weak interaction. Leille Lopes, in analogy to the Yukawa theory, guessed that there may be a neutral IVB along with the two charged ones that, and that their masses were $\mathcal{O}\left(50 \mathrm{GeV} / c^{2}\right)[15]$. The $S U(2)$ group was recognized as a fundamental symmetry for the weak interaction, and Glashow used an $S U(2) \otimes U(1)$ framework to explain both the weak and electromagnetic forces[16]. The $S U(2)$ group has three generators and so there were three associated massless gauge fields. One could add mass terms by hand, but this would break the gauge symmetry and render the theory non-renormalizable. The solution to this problem would come in the form of "spontaneous symmetry breaking". We proceed to describe some aspects of the (massless) SM before discussing spontaneous symmetry breaking.

\subsubsection{Matter Fields}

In the SM, matter is composed of three generations of quarks and leptons and their antiparticles. All other matter is composed of these fundamental particles. The protons and neutrons within atomic nuclei, for example, are composed of three quarks each, and the electron is a first-generation lepton. Due to the experimental fact that the $W^{ \pm}$only couple to fermions of left helicity, the left- and right-handed components of the fermion fields have different representations in the SM. Also, the neutrinos are assumed to be identically massless and left-handed.

\footnotetext{
${ }^{10}$ The left-handed and right-handed components of Dirac fields are given by $\psi_{L / R}=\frac{1}{2}\left(1 \pm \gamma_{5}\right) \psi$ More information the Dirac formalism and spin can be found in the usual texts[4, 5, 6].

${ }^{11}$ Neutrinos are discussed in a little more detail in Appendix B.
} 


$$
\begin{aligned}
L_{1}=\left(\begin{array}{c}
\nu_{e} \\
e
\end{array}\right)_{L}, \bar{e}_{R} ; Q_{1}=\left(\begin{array}{l}
u \\
d
\end{array}\right)_{L}, u_{R} d_{R} \\
L_{2}=\left(\begin{array}{c}
\nu_{\mu} \\
\mu
\end{array}\right)_{L}, \bar{\mu}_{R} ; Q_{2}=\left(\begin{array}{c}
c \\
s
\end{array}\right)_{L}, c_{R} s_{R} \\
L_{3}=\left(\begin{array}{c}
\nu_{\tau} \\
\tau
\end{array}\right)_{L}, \bar{\tau}_{R} ; Q_{3}=\left(\begin{array}{c}
t \\
b
\end{array}\right)_{L}, t_{R} b_{R}
\end{aligned}
$$

\subsubsection{Gauge Bosons}

As we saw in the QED example, gauge bosons result from the process of "patching" the derivatives to make them covariant under gauge transformations. The symmetry transformations that describe the strong and weak forces are more complicated than the simple $U(1)$ phase shift of QED. For example, the strong interaction is described by an $S U(3)$ gauge theory. There are eight generators of transformations for the $S U(3)$ group, and the covariant derivative requires eight fields, which correspond to the eight "gluons" that mediate the strong force. The electromagnetic and weak interactions are described by and $S U(2) \otimes U(1)$ framework, where the $\otimes$ represents a direct product. The gauge bosons corresponding to the group generators are the massive $W^{+}, W^{-}, Z$, and the massless photon.

\subsubsection{Electroweak Unification}

The electromagnetic and weak forces are described in an $S U(2)_{L} \otimes U(1)$ framework. where the $\otimes$ represents a direct product - the generators of the $S U(2)_{L}$ and $U(1)$ transformations must commute so that they can be simultaneous symmetries of the Lagrangian. The subscript $L$ on $S U(2)_{L}$ indicates that only left-handed fermions partake in the weak interaction. The generators of the $S U(2)$ transformations are $T_{i} \equiv \frac{1}{2} \tau_{i}$, where $\tau_{i}$ are the Pauli spin matrices. There are three conserved quantities associated with the generators $T_{i}$, and they are known as the three components 
of "Weak Isospin". The electric charge operator $Q$ of $U(1)_{E M}$ does not commute with the generators of the $S U(2)$ transformations. A quantity called the "Weak Hypercharge", defined by

$$
Y=2\left(Q-T_{3}\right)
$$

commutes with $T_{i}$ and demonstrates the connection of the electromagnetic and weak interactions in the $S U(2) \otimes U(1)$ framework.

\subsubsection{The Unification of the Strong and Electroweak Interactions}

The Standard Model describes the three interactions in an $S U(3) \otimes S U(2) \otimes U(1)$ framework. The unification of the strong and electroweak interaction involves a direct product. The generators of the $S U(3)$ transformations associated with the strong force commute with the electroweak generators, so there is no relationship analogous to $(1.11)^{12}$.

\subsubsection{The Higgs Mechanism}

The Higgs Mechanism involves an idea called "Spontaneous Symmetry Breaking". The theory is assumed to have some symmetry that is not observed in the vacuum. For example, consider a scalar field $\phi$ (called the Higgs field in the $\mathrm{SM}^{13}$ ) in a potential $V(\phi)=\frac{1}{2} \mu^{2} \phi^{2}+\frac{1}{4} \lambda \phi^{4}$. The potential's behavior depends on the signs of $\mu^{2}$ and $\lambda$. First let's consider the case $\lambda>0, \mu>0$, as shown in Figure 1.1. The potential has one global minimum. Small fluctuations about the vacuum are of $2^{\text {nd }}$ order in $\phi$, and correspond to a mass of $\mu^{2}$ for the scalar field $\phi$. If, on the other hand, we take $\mu^{2}<0$, as in Figure 1.2, and expand around $\phi=0$, we get a particle of negative mass. However, $\phi=0$ is not the vacuum - the potential has two minima at $\phi^{ \pm}= \pm \sqrt{-\mu^{2} / \lambda}$. We must pick either $\phi^{+}$or $\phi^{-}$as our vacuum, thereby breaking the symmetry. The vacuum takes on an expectation value of $v=\sqrt{-\mu^{2} / \lambda}$.

\footnotetext{
${ }^{12}$ However, it is not fair to say that the strong and electroweak interactions are unified in a trivial way. Both interactions are necessary to cancel triangle anomalies.[7]

${ }^{13}$ Except when referred to by Peter Higgs, who still calls it "the scalar field".
} 
We can define a new field $\tilde{\phi} \equiv \phi-v$ so that $<\tilde{\phi}>=0$ and can again consider small oscillations around the vacuum state. In terms of the new field $\tilde{\phi}$, the Lagrangian is

$$
\mathcal{L}=\frac{1}{2} \partial_{\mu} \tilde{\phi} \partial^{\mu} \tilde{\phi}-\frac{1}{2}\left(\sqrt{-2 \mu^{2}}\right)^{2} \tilde{\phi}^{2}-\lambda v \tilde{\phi}^{3}-\frac{1}{4} \lambda \tilde{\phi}^{4}
$$

which is no longer symmetric due to the $\tilde{\phi}^{3}$ term. However, we do see that the field $\tilde{\phi}$ has a positive mass of $\sqrt{-2 \mu^{2}}$. In the SM implementation of spontaneous symmetry breaking, the analog to this scalar is called the Higgs boson.

Consider next a complex scalar field $\phi=\phi_{1}+i \phi_{2}$ and the potential in Figure 1.3. The potential has infinite degenerate minima that lie in a circle. We are free to choose as the vacuum any point that lies on that circle, but we break the rotational invariance by doing so. Let the vacuum to be a particular point $\left(v_{1}, v_{2}\right)=(v, 0)$, and use the field $\tilde{\phi}=\left(\tilde{\phi}_{1}, \tilde{\phi}_{2}\right)=\left(\phi_{1}-v+i \phi_{2}\right)$ to represent fluctuations about the vacuum. We see that the field $\tilde{\phi}_{1}$, the component in the radial direction, has acquired mass, while $\tilde{\phi}_{2}$ remains massless - it's a Nambu-Goldstone Boson. The Goldstone theorem ${ }^{14}$ states that, after symmetry breaking, there will be a massless boson for each broken generator of the original symmetry. The field $\tilde{\phi}$ can be approximated to first order in small oscillations as

$$
\tilde{\phi}=\left(\tilde{\phi}_{1}, \tilde{\phi}_{2}\right)\left(\phi_{1}-v+i \phi_{2}\right) \simeq e^{i \frac{\phi_{2}}{v}}\left(\phi_{1}-v\right)=\phi_{1}-v+i \phi_{2}+\mathcal{O}\left(\phi^{2}\right) .
$$

With this parameterization, it is easy to "gauge away" the Goldstone Boson $\tilde{\phi}_{2}$, by the gauge transformation $U=e^{-i \frac{\tilde{\phi}_{2}}{v}}$ - after all, the Lagrangian is invariant under such transformations. We are left with $\phi=\tilde{\phi}_{1}+v$.

A more interesting case occurs when the symmetries of the Lagrangian are local. We have seen that massless gauge fields $A_{\mu}$ are needed to maintain invariance of the Lagrangian. The substitution for $\phi: \phi=\tilde{\phi}_{1}+v$ results in the following kinetic

\footnotetext{
${ }^{14} \mathrm{~A}$ short proof and even shorter commentary is provided the Appendix. A.
} 
term associated with the scalar field:

$$
D_{\mu} \phi D^{\mu} \phi \rightarrow\left(\partial_{\mu}+i q A_{\mu}\right)\left(\tilde{\phi}_{1}+v\right)\left(\partial^{\mu}+i q A^{\mu}\right)\left(\tilde{\phi}_{1}+v\right)
$$

One can see from terms like $v^{2} A_{\mu} A^{\mu}$ that the gauge fields have acquired mass! Likewise, couplings between the scalar and fermion fields result in the fermion masses. Although the gauge symmetry is broken in the ground state, it has been proven by 't Hooft and Veltman that it is still possible to renormalize gauge theories with spontaneously broken symmetries[17]. This means that we now have a renormalizable gauge theory with massive gauge bosons!

Note that the degrees of freedom (DOF) associated with the massless NambuGoldstone Bosons are transferred to the now-massive gauge bosons. Before symmetry breaking, we have a complex scalar field $\phi=\left(\phi_{1}+i \phi_{2}\right)$ with two degrees of freedom, and a massless gauge field $A_{\mu}$ also with two degrees of freedom. After symmetry breaking, we have a real scalar $\tilde{\phi}=\left(\phi_{1}+v\right)$, with one degree of freedom, and a now massive $A_{\mu}$ with three degrees of freedom. The degree of freedom associated with $\tilde{\phi}_{2}$, which has been gauged away, is now the longitudinal polarization of the massive field $A_{\mu}$. The general case is summarized in Table 1.2. For the Standard Model, we need to have three degrees of freedom transferred to the gauge sector. The simplest way to do this is to start out with a scalar field with four degrees of freedom, such as a complex doublet, and end up with one massive scalar, three massive gauge bosons $\left(W^{+}, W^{-}, Z\right)$, and a massless $\gamma$. The photon's masslessness reflects the unbroken $U(1)_{E M}$ symmetry. The symmetry breaking scheme is summarized as $S U(2)_{L} \otimes U(1)_{Y} \rightarrow U(1)_{E M}$. The Higgs mechanism relates the masses of the $\mathrm{W}$ and $\mathrm{Z}$ bosons, and this relationship, expressed in terms of the $\rho$ parameter

$$
\rho=\frac{m_{W}^{2}}{m_{Z}^{2} \cos ^{2} \theta_{w}} \equiv 1 \text { (at tree level) }
$$

where $\cos \theta_{w}$ is the weak mixing angle, and can be determined from the mass of the 


\begin{tabular}{|c|c|}
\hline DOF Before Symmetry Breaking & DOF After Symmetry Breaking \\
\hline$\phi$ (massless scalar): $N_{\phi}$ & $\dot{\phi}$ (massive scalar): $N_{\phi}-\left(N_{I}-N_{F}\right)$ \\
$A_{\mu}^{i}$ (massless vector fields): $2 \times N_{I}$ & $A_{\mu}^{i}$ (massive vector fields): $3 \times\left(N_{I}-N_{F}\right)$ \\
& $A_{\mu}^{i}$ (massless vector fields): $2 \times N_{F}$ \\
\hline
\end{tabular}

Table 1.2: Counting the degrees of freedom before and after symmetry breaking. $N_{\phi}$ is the dimensionality of the original scalar field. $N_{I}$ is the number of initial (unbroken) symmetries, and $N_{F}$ is the number of symmetries that remain after the symmetry breaking. The sum for both columns is $N_{\phi}+2 \times N_{I}$.

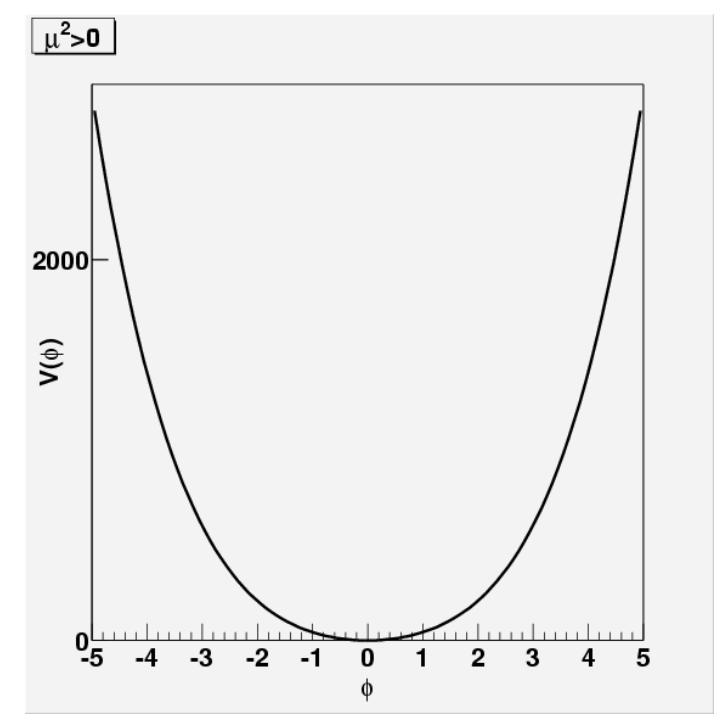

Figure 1.1: Potential $V(\phi)=\frac{1}{2} \mu^{2} \phi^{2}+\frac{1}{4} \lambda \phi^{4}$ with $\mu^{2}>0, \lambda>0$

Z-boson, the Fermi coupling constant, and the fine structure constant ${ }^{15}$. Agreement between data and the SM prediction of the $\rho$ parameter is quite good[18], and guides the phenomenology of theories that contain extended Higgs sectors. The Higgs boson, is the only member of the SM that has yet to be directly observed.

\subsubsection{Remarks on the SM}

The unification of the strong, electromagnetic and weak interactions is an obvious success of the SM - such scope, economy, and elegance is rare among idealogical frameworks that actually abide by experimental constraints. The theory's predic-

\footnotetext{
${ }^{15}$ These are just one choice for the set of three independent parameters needed to describe the electroweak sector.
} 


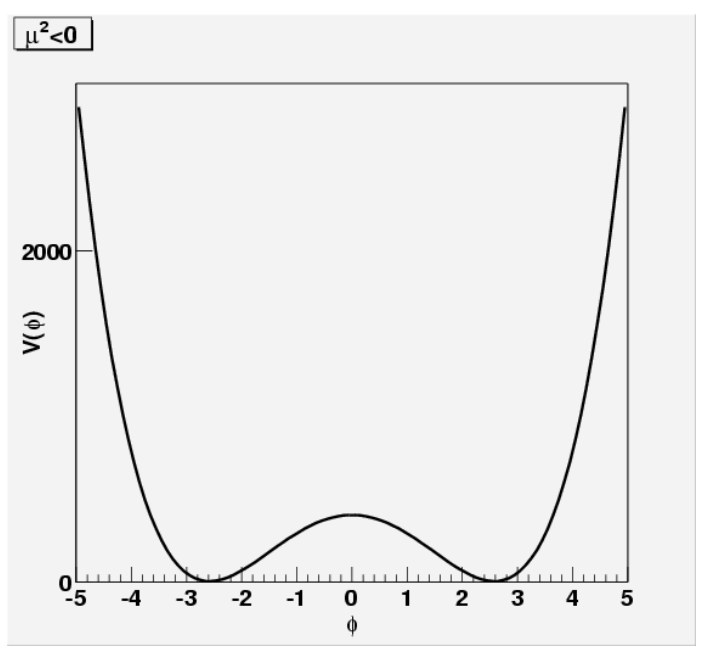

Figure 1.2: Potential $V(\phi)=\frac{1}{2} \mu^{2} \phi^{2}+\frac{1}{4} \lambda \phi^{4}$ with $\mu^{2}<0, \lambda>0$

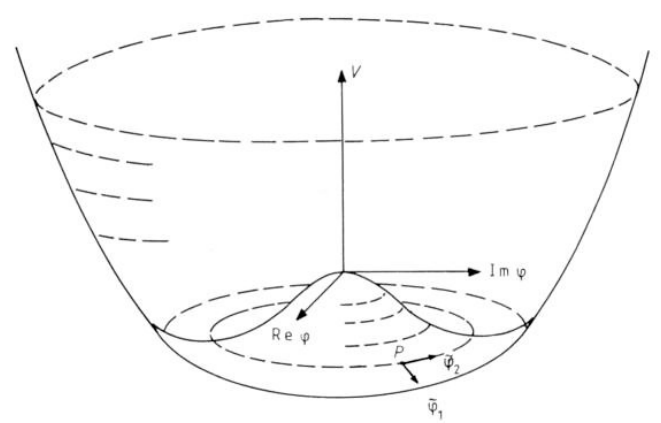

Figure 1.3: The 3-D generalization of the potential given in figure 1.2.

tive power is also exemplary. The $W$ and $Z$ bosons, gluons, the top and charm quarks, and the tau neutrino were all predicted to exist by the SM before they were discovered by experimentalists. Furthermore, the particles possessed all the properties that the theory predicted ${ }^{16}$ The SM has been found to be consistent with many precision tests in addition to the $\rho$ parameter. For example, there is the "invisible" decay width of the $Z$, which can be related to the number of (weakly interacting) neutrino generations with masses $m_{\nu}<m_{Z} / 2$. Experiments at LEP and SLAC have measured that there are $2.994 \pm 0.011$ generations of such neutrinos. QCD predictions on the total inelastic cross sections of colliding beams are also in very good

\footnotetext{
${ }^{16}$ With the exception of the neutrino, as discussed later.
} 
agreement with data. The agreement between experiment and QED calculations regarding the anomalous magnetic moment of electrons is staggering - the results are identical to 10 significant digits[19]!

Despite the SM's successes, it is widely hoped that the Standard Model will be incorporated into, or replaced by, a grand unification theory (GUT). The very success of the SM makes one wonder about the possibility of having a theory with just one coupling constant, that describes all four interactions and their relationship from the high energy scale associated with the beginning of the universe ${ }^{17}$ down to the energies that we probe in experiments. The SM does not have anything specific to say about phenomena at the GUT scale, and it does not incorporate the gravitational interaction. Furthermore, the model has 19 free parameters, which is considered to be way too many! There is also hierarchy problem, which has to do with corrections to the Higgs mass from all fields - if there are any fields at the GUT-scale, then the higher order corrections to the Higgs mass would be of such large magnitudes that it would take an unnatural level of cancellation between the correction terms to keep the Higgs mass low enough such that the model respects unitarity.

During the 1950's and 1960's, experiments discovered many new particles and new phenomena like parity- and CP-violation. In some sense, the theorists were playing "catch-up". The Standard Model kept the experimentalists busy with decades of discoveries and precision measurements, while theorists had time to address the issues discussed above. So, when neutrino experiments started to confirm long running suspicions ${ }^{18}$ that neutrinos oscillated between states of different flavors, and were therefore massive, there were already several frameworks that predicted massive neutrinos. The Standard Model can be made to accommodate massive neutrinos - the addition of a right-handed neutrino field results in Dirac masses just as with

\footnotetext{
${ }^{17}$ The relevant energy scales are the GUT scale, $\left(10^{16} \mathrm{GeV}\right)$, where the strengths of the three forces become similar, and the Planck scale $\left(10^{19} \mathrm{GeV}\right)$, where the gravitational coupling has similar strength to the other couplings.

${ }^{18}$ The "solar neutrino deficit" was first observed in 1964.
} 
the other fermions, but, given the aesthetics ${ }^{19}$ discussed above, the "patch" will at be regarded as "kludgy". There are extensions of the Standard Model that can explain the neutrino masses much more naturally. We briefly discuss two such theories below.

\footnotetext{
${ }^{19}$ It's not just aesthetics, it's good intuition based on the historical advancement of knowledge.
} 


\subsection{The Left-Right Symmetric Model}

In the Left-Right Symmetric Model (LRSM), the Lagrangian possesses the symmetry $S U(3)_{\text {color }} \otimes S U(2)_{L} \otimes S U(2)_{R} \otimes U(1)_{Y}$. The new symmetry, $S U(2)_{R}$, is identical to the familiar $S U(2)_{L}$, except that it only transforms right-handed components of fermions. Above the $S U(2)_{R}$ breaking scale (which is necessarily higher then the electroweak symmetry-breaking scale), there is no parity violation. The model predicts gauge bosons that couple to right handed leptons, and, right-handed neutrinos $\nu_{R}$ - thus there can be a Dirac mass term for the neutrinos. In fact, the mass of the left-handed neutrinos are related to the masses of the new right-handed particles $^{20}$ by the "see-saw mechanism" ${ }^{21}$, so that the lightness of the left-handed neutrino is related to the suppression of the right-handed weak current (i.e. the large masses of $W_{R}$ ). The seesaw mechanism, along with our present understanding of the $\tau$ and $\nu_{\tau}$ masses, would put the masses of the $S U(2)_{R}$ particles at the GUT scale. Fittingly, the LRSM is readily incorporated into larger GUT theories, such as $S O(10)$ or $S U(7)$. The LRSM has an enhanced Higgs sector: in addition to the complex scalar doublet, there are two complex scalar triplets. There are 16 degrees of freedom between the scalar doublet and triplets before symmetry breaking, as opposed to four for the complex doublet of the SM. After breaking of $S U(2)_{R}$, three degrees of freedom become longitudinal DOF for the massive gauge bosons $W_{R}, Z_{R}$, likewise, three DOF go to the standard electroweak sector. That leaves 10 DOF left over to the Higgs sector. The scalars are:

$$
H_{L}^{++}, H_{L}^{--}, H_{R}^{++}, H_{R}^{--}, H_{L}^{+}, H_{L}^{-}, H_{R}^{+}, H_{R}^{-}, H^{0}, h^{0}
$$

At the Tevatron, the doubly charged Higgs, $H^{ \pm \pm}$, would be pair-produced, and would likely decay into four high-energy leptons. Experimental observation of the

\footnotetext{
${ }^{20}$ One can assume that the new gauge bosons and neutrinos are of the same mass scale. Also, since neutrinos have mass, they can't be exactly right- or left- handed, but only mostly so. For more information see Appendix C.

${ }^{21}$ The seesaw mechanism is discussed in Appendix C
} 
$H^{ \pm \pm}$would be a spectacular confirmation of the LRSM.

One of the most stringent experimental limits on many theories with enhanced Higgs sectors comes from the $\rho$ parameter. Additional Higgs triplets, for example, can change the $W$ mass while leaving the $Z$ mass untouched, and make $\rho$ deviate from unity. The LRSM can stay safely within experimental bounds by adjusting ${ }^{22}$ the vacuum expectation values (VEVs) of the Higgs fields. This tends to suppress the coupling of the left handed Higgs triplet to the $W_{L}^{ \pm}$bosons. This is good for experimentalists because it results in large branching ratios of $H^{ \pm \pm}$to leptons ${ }^{23}$, making for clean experimental signatures.

\subsection{Higgs Triplet Model}

The Higgs Triplet Model (HTM) extends the SM Higgs sector with the addition of a complex Higgs triplet. It does not assume spontaneous parity nonconservation as with the LRSM. The neutrinos become Majorana particles (their own antiparticles), so that all neutrinos can still be left-handed and all antineutrinos can remain right-handed. The Majorana nature of the neutrinos, however, implies lepton flavor violation $\Delta l= \pm 2$. This isn't necessarily bad - lepton flavor conservation does not follow from an application of Noether's theorem - it is simply an assumption based on our experience with more common occurrences.

The triplet of the HTM is similar to the left- and right-handed triplets in the LRSM:

$$
\Delta=\left(\begin{array}{cc}
\Delta^{+} / \sqrt{2} & \Delta^{++} \\
\Delta^{0} & -\Delta^{+} / \sqrt{2}
\end{array}\right)
$$

shown above in a $2 \times 2$ representation ${ }^{24}$. The HTM can be consistent with $\rho=1$ by having a small $\mathcal{O}(10 \mathrm{keV}) \mathrm{VEV}, v_{\Delta}$, for the neutral member of the triplet $\Delta^{0}$. The

\footnotetext{
${ }^{22}$ But not tuning - the theory can have its members' masses adjusted by many hundreds of $\mathrm{GeV} / c^{2}$ while remaining consistent with $\rho-1$ measurements.

${ }^{23}$ There's no way for the $H^{ \pm \pm}$to couple to two quarks and conserve electric charge.

${ }^{24}$ There are three independent complex fields here, $\Delta^{0}, \Delta^{+}$, and $\Delta^{++}$.
} 
(Majorana) mass matrix for the neutrinos has entries $m_{i j}=\sqrt{2} h_{i j} v_{\Delta}$, where $h_{i j}$ are arbitrary Yukawa couplings. Note that the necessary smallness of $v_{\Delta}$ implies small neutrino masses. The Higgs sector of the HTM has 10 DOF. As usual, three are transferred to the electroweak gauge sector, leaving us with seven Higgs bosons:

$$
H^{++}, H^{--}, H^{+}, H^{-}, h^{0}, H^{0}, A^{0}
$$

As with the LRSM, the most sensational new members are the doubly-charged $H^{ \pm \pm}$, which are likely to decay mainly in to like-sign dileptons of unrestricted flavors. 


\section{Chapter 2}

\section{Experimental Apparatus}

\section{$2.1 \quad$ Beam}

There are two sources of very high energy particles for high energy physicists to study: cosmic rays and particle beams. Cosmic rays can have energies far beyond what can be created in the lab. It is very challenging, however, to scrutinize the atmospheric interactions of cosmic rays from afar. Nevertheless one can reconstruct the flux and momenta of the incoming particles and relate the information to happenings far off in the cosmos. Cosmic rays of very high energies are extremely rare and thus, related experiments suffer from low event rates. Alternatively, high energy physicists create and collide beams of particles in the laboratory, and analyze collisions' outcomes in detail with the aid of computers and other sophisticated instrumentation. This approach has yielded historic successes - the discoveries and precision measurements of many new and rare processes. In discovery-driven collider experiments, two paramount beam parameters are the available center of mass $(\mathrm{CM})$ beam energy $\sqrt{s}^{[1]}$ and the flux of particles through the interaction region of the two beams, known as the luminosity, $L$. The event rate $R$ for a given process is given by $R=L \sigma_{\text {interaction }}$, where $\sigma_{\text {interaction }}$ is the "cross section" for a particular

\footnotetext{
${ }^{1}$ Here $s$ is the Mandelstam variable $s \equiv\left(p_{1}^{\mu}+p_{2}^{\mu}\right)^{2}$ where $p_{1}^{\mu}$ and $p_{2}^{\mu}$ are the 4 -vectors of the incoming beam particles.
} 
process to occur. The luminosity is given by the formula

$$
L=f \frac{n_{1} n_{2}}{4 \pi \sigma_{x} \sigma_{y}}
$$

where $\sigma_{x}$ and $\sigma_{y}$ are the beam profiles (the widths of Gaussian distributions) in the transverse directions and $n_{1}$ and $n_{2}$ are the numbers of particles in the colliding "bunches" of beam, and $f$ is the frequency of collisions. To achieve high luminosity, it is necessary to minimize $\sigma_{x}$ and $\sigma_{y}$ by "cooling" (discussed later) the beam and focusing the beam with magnets, while maximizing $f, n_{1}$, and $n_{2}$.

\section{$2.2 \quad$ Particle Accelerators}

Particle accelerators use electric fields to accelerate charged particles. The earliest ones, such as the Cockroft-Walton[20] machine, employ electrostatic fields. A charged particle traversing an electric field gains momentum according to the equation

$$
\frac{\partial \vec{p}}{\partial t}=q \vec{E}
$$

This method produced the first artificial nuclear reaction $\left(L i^{7}+p \rightarrow H e^{4}+H e^{4}\right)$ in 1932fnal, and is now in ubiquitous use accelerating electrons in cathode ray tubes. The energies attained by such a scheme are limited by dielectric breakdown in the acceleration chamber. One solution to this problem is to use several accelerators in series. Linear accelerators (linacs) utilize an array of acceleration units arranged in a linear fashion. A particle traveling through the linac gains a kinetic energy of $E_{0}$ at each unit, and emerges with energy $E_{\text {Linac }}=N \times E_{0}$, where $N$ is the number of acceleration units in the linac. Another class of accelerators confines the particles in a closed, roughly circular path that includes one or more acceleration stations. Particles in stable orbits can stay in the loop for many circuits and gain energy at each cycle. Magnetic fields are used to bend the particles around the loop. These magnetic fields must be increased as the particle beam gains energy. The particles, 
electric fields, and bending magnetic fields must all be "in synch" for the machine to work properly, thus the name "synchrotron" applies to this class of accelerators. One by-product of the centripetal acceleration of the particles is the emittance of "synchrotron radiation". The power radiated is given by the Larmor formula

$$
P=\frac{1}{6 \pi \epsilon_{0}} \frac{e^{2} a^{2}}{c^{3}} \gamma^{4}
$$

where $\gamma$ is the relativistic factor, $e$ is the electric charge of the beam particle, $a$ is the acceleration of the particle, $c$ is the speed of light in a vacuum, and $\epsilon_{0}$ is the permittivity of free space. This radiation can be a good source of bright UV and x-ray photons, but is problematic for the particle physicist: the radiation increases dramatically with beam energy for a given radius. At some point it becomes impractical to raise beam energies further, because most of the added energy is radiated away. For example, the amount of energy needed to compensate the $100 \mathrm{GeV}$ beam at the LEP collider (with a $27 \mathrm{~km}$ circumference ring) is about $2 \mathrm{GeV}$ per turn. If the beam were to be doubled in energy at the same radius, then $35 \mathrm{GeV}$ of energy would have to be restored per turn. The energy scale attainable by synchrotrons for electron beams is therefore limited by synchrotron radiation for any practicable radius. For proton beams, however, synchrotron radiation is not significant, as there is in implicit $\frac{1}{(\text { mass })^{4}}$ term in (2.3). For comparison, the $1 \mathrm{TeV}$ protons in the Tevatron, which has a radius of $1 \mathrm{~km}$, radiate only $7.8 \mathrm{eV}$ per turn. The high magnetic fields required to confine beams to the synchrotron's path are also a limiting factor. The magnetic field from iron electromagnets is limited, so superconducting magnets must be used to sustain the required fields (4.5 Tesla at the Tevatron at temperatures of $4.6 \mathrm{~K}$, and almost twice that at the LHC at temperatures of $1.9 \mathrm{~K})$. Current superconducting magnets cannot reliably sustain fields above 10 Tesla. Two possible solutions are improvements in superconductor technology and larger (so more expensive) synchrotron rings.

The basic tool for accelerating particles in these accelerators is a resonant fre- 
quency (RF) cavity. A resonant cavity has surfaces that reflect an electromagnetic wave. The shape and size of the cavity can be tuned to obtain standing waves of desired frequencies (typically in the radio range) and configurations. Figure 2.1 shows a cross section of an RF cavity. To obtain a net accelerating field, the particles must only experience positive electric forces in the direction of motion. One solution is to shield the particles from the negatively aligned fields. This is done with "drift tubes" that insulate the particles from the opposing fields. A particle of the proper phase passes through gaps in the drift tubes (called accelerating gaps) only when the field is in the desired direction. Successive drift tubes and acceleration gaps must be made longer since the particle covers more and more distance in a given RF cycle as it accelerates. This scheme is depicted in Figure 2.2.

Another method is to have "side coupled" RF cavities that are out of phase by such an amount that a particle always sees positive fields as it traverses many accelerating cells. Figure 2.3 depicts a particle traversing a pair of side-coupled $\mathrm{RF}$ cavities. If some particles are sufficiently in phase with the RF cavities in an accelerator, then the forces they experience tend to keep them in phase with the RF. Particles that are ahead in time tend to experience a slightly smaller accelerating force than particles that are behind, and vice versa. Figure 2.4 illustrates this process. A group of particles that have a stable orbit because they are sufficiently in phase with the RF accelerating field is called a "bunch". A proton bunch at the Tevatron typically has about 230 billion protons, while an antiproton bunch has about one fourth that number. Each bunch has a momentum spread and a time spread. A 2-D phase space diagram can be made by plotting $\delta E / E$ vs $\delta t / t$ or simply the phase angle $\phi$. The area in phase space that the stable particles occupy is called a "bucket", although a bucket can also refer to the same area in phase space - the effective wavefront of the accelerating field - whether there is beam there or not. The momentum spread and time spread can be manipulated by varying the RF voltages ${ }^{2}$. The "density" in the phase space is incompressible for adiabatic

\footnotetext{
${ }^{2}$ Here we refer to the momentum along the beam direction. There are ways to manipulate the
} 


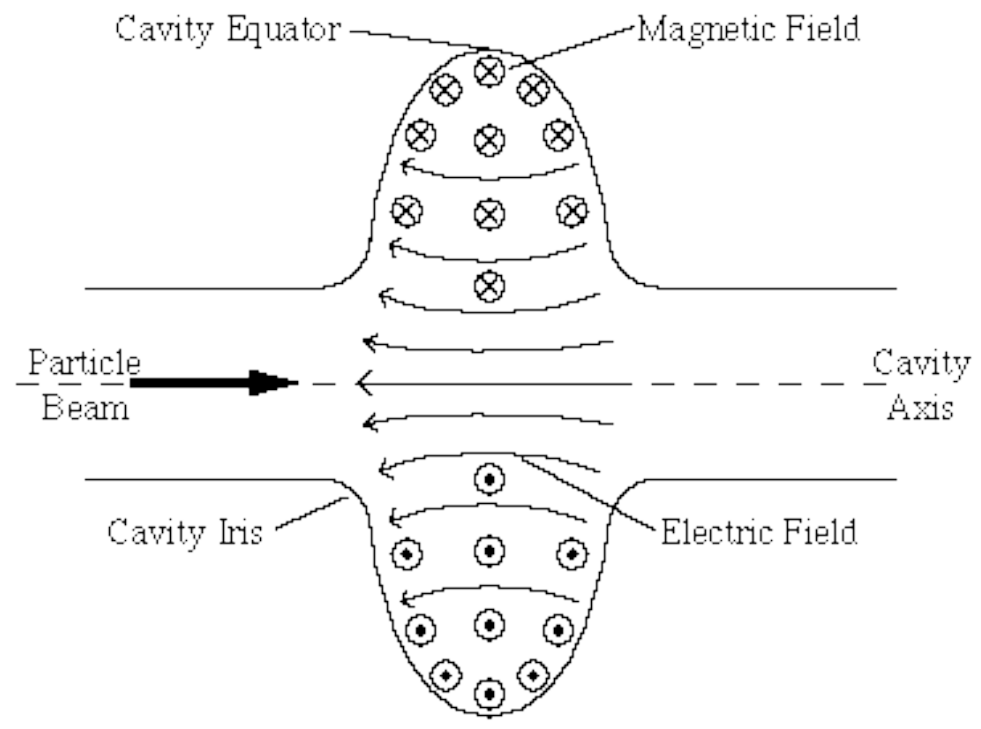

Figure 2.1: A cross section of an RF cavity ${ }^{4}$. The beam experiences an electric field that oscillates between parallel and antiparallel to its direction. If the particle can be shielded from the antiparallel fields, then it can experience a net accelerating force.

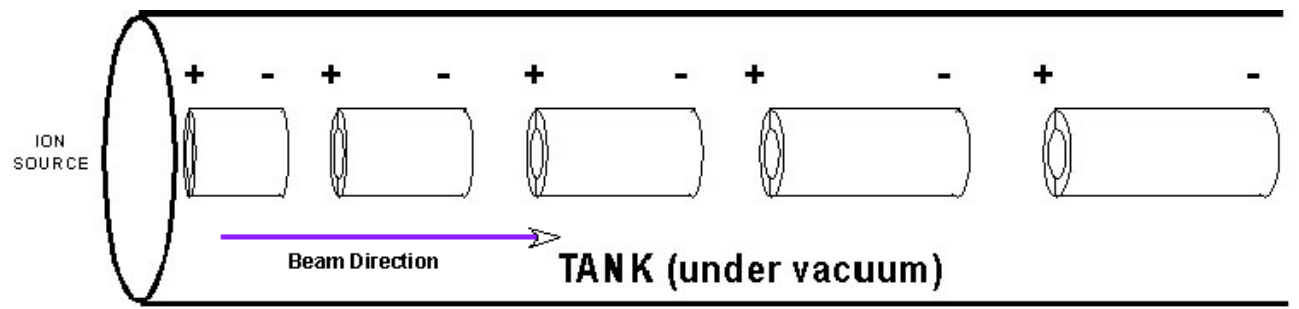

Figure 2.2: A cross section of a long RF cavity with several drift tubes inside. A particle that is in phase will experience electric fields only when it is in the accelerating gaps.

processes, according to Liouville's theorem, thus a narrowing of the time-spread is done at the cost of widening the momentum-spread.

particles' momenta in the direction transverse to the beam as well. Some of these methods are described later in this section.

${ }^{4}$ All figures of accelerator components were taken from [20] 

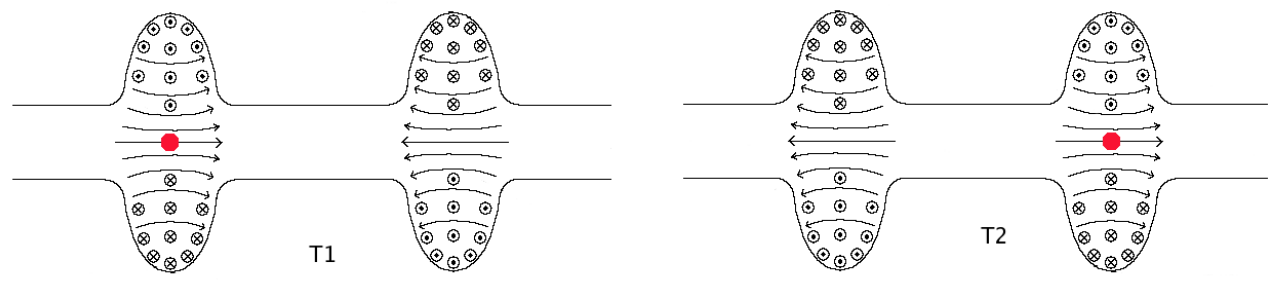

Figure 2.3: A schematic representation of side-coupled RF cavities. These cavities are phase-shifted with one another so that a particle of the proper phase (depicted by the solid circle) will always experience positive electric fields in the direction of motion.

\section{$2.3 \quad$ Tevatron}

The Tevatron complex[20] is an series of accelerators capable of accelerating protons and antiprotons to energies of $980 \mathrm{GeV}$ and colliding them at a center-of-mass (CM) energy of $\sqrt{s}=1.96 \mathrm{TeV}$, hence the name, and is currently the highest energy particle collider in the world.

The Tevatron itself is a $1-\mathrm{km}$ radius synchrotron. The synchrotron magnets are made of a superconducting niobium/titanium alloy, which are maintained at $4.6 \mathrm{~K}$ with liquid Helium during operations. The superconducting material can sustain the 4.6 Tesla magnetic fields required to keep the beam in a circular path. Ohmic materials would suffer from high power consumption and resistive heating and would not be able to safely maintain fields of adequate strength. Before entering the Tevatron, protons and antiprotons must go through various stages of acceleration. A schematic diagram of the accelerator complex is given in Figure 2.5.

\subsubsection{Protons}

The first stage of acceleration for the protons occurs in the Cockroft Walton electrostatic accelerator. Protons start as hydrogen gas $\left(H_{2}\right)$, and are stored at a potential of $-750 \mathrm{kV}$. The gas is ionized to $H^{-}$by picking up extra electrons, and acceler- 

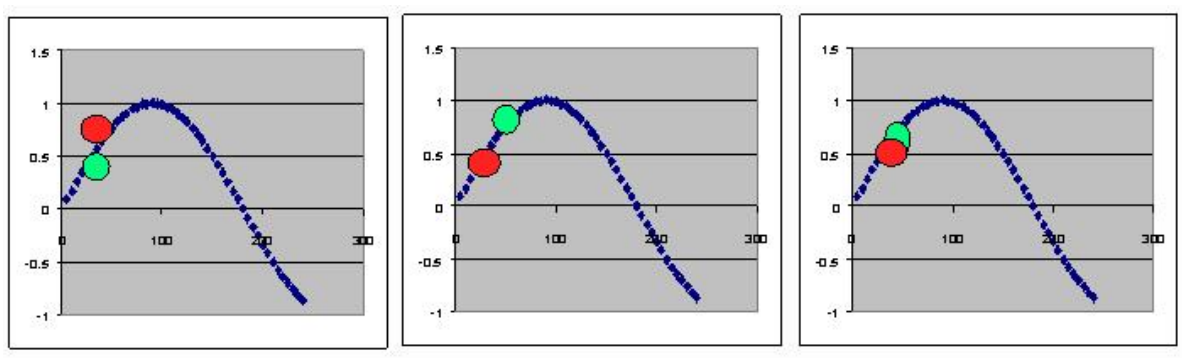

(a)

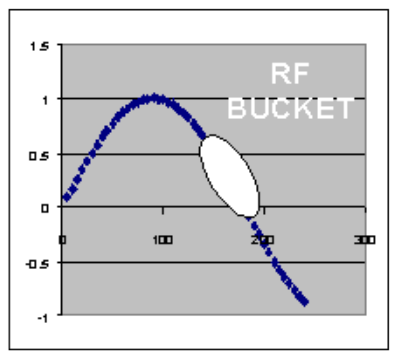

(b)

Figure 2.4: (a) The leftmost figure shows two particles arriving at the same time(horizontal axis) to an RF station in a linac, where they experience the same accelerating force - as represented by the amplitude of the standing wave. Assume the dark-colored particle is initially more energetic than the light-colored one. At the next RF station, the dark-colored particle arrives sooner, thus experiences a smaller force than the light-colored one (each plot shows two particles at the same location -the RF cavity - but at different times and corresponding RF amplitudes. Time increases to the right.), thus the light-colored particle can "catch up". In this way, particles that are nearly synchronous with the RF field can maintain a stable orbit. (b) A stable bunch of particles in a circular synchrotron. Notice the phase of the particles is different than before - they are now "ahead" of the RF wave.

ates toward and a passing through a small hole in a wall that is grounded, thereby obtaining a kinetic energy of $750 \mathrm{KeV}$. The $H^{-}$ions are then passed to a linear accelerator (linac)[20] in $40 \mu s$ segments. There the beam segments are accelerated to $116 \mathrm{MeV}$ in a series of drift tube RF cavities operating at $201 \mathrm{MHz}$, and then further accelerated to $400 \mathrm{MeV}$ by a series of side-coupled RF cavities operating at $805 \mathrm{MHz}$. After the linac phase the beam is fed in $20 \mu s$-long segments to a $75 \mathrm{~m}$ radius synchrotron, called the Booster[20], that accelerates them to $8 \mathrm{GeV}$. The $\mathrm{H}^{-}$ 


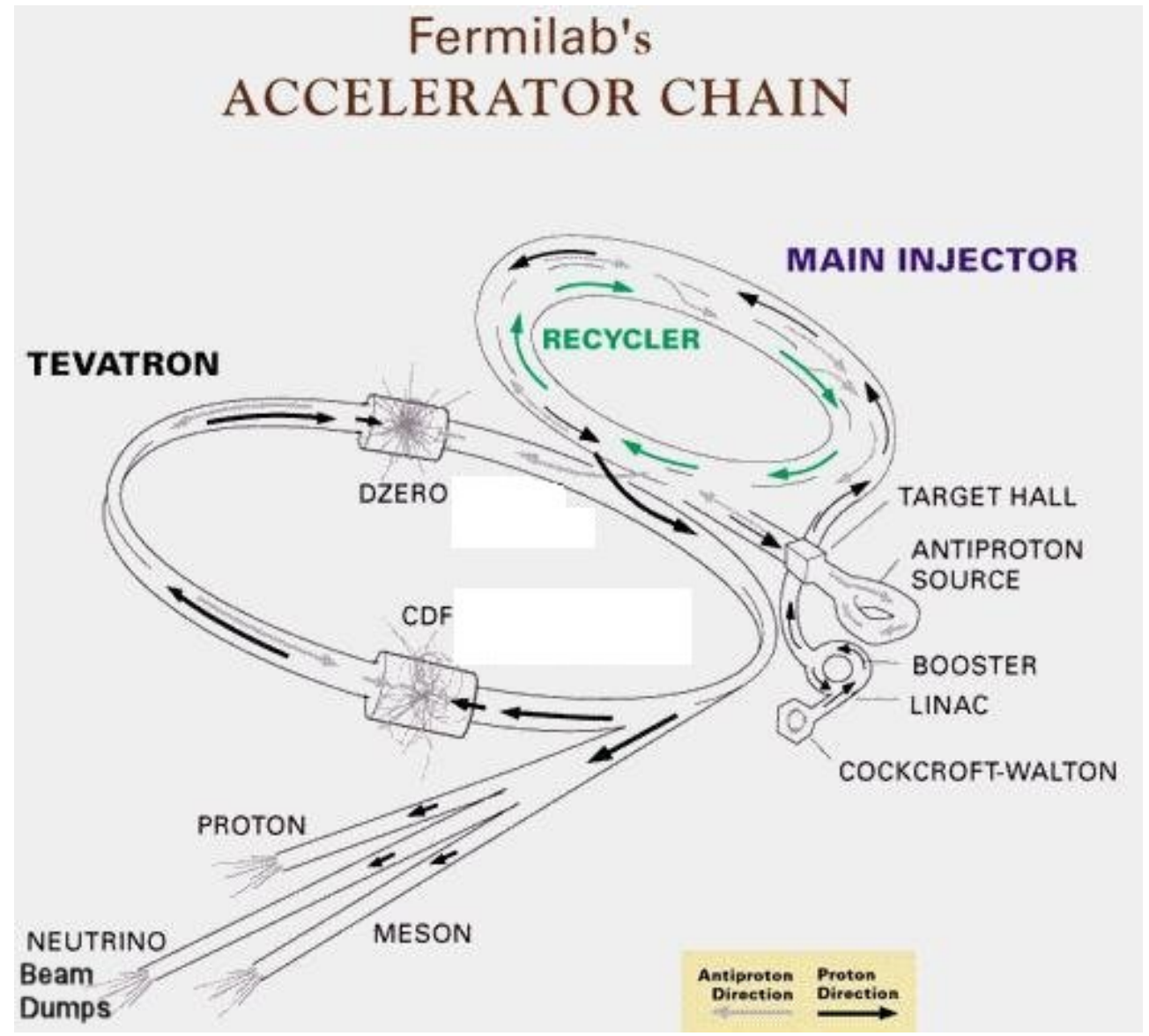

Figure 2.5: A schematic representation of the Tevatron Complex.

must be converted to protons upon entering the Booster. The incoming beam from the linac is composed of relatively long pulses of $\mathrm{H}^{-}$which wrap around the booster several times. The $H^{-}$beam approaches the Booster in a nearly tangential path. The negatively charged ions bend in the opposite direction as the protons do in the Booster's magnetic field and pass through eight carbon foils which strip off the electrons, leaving protons. At this point the beam is merged with the preexisting beam in the Booster, and all the protons follow the same path. In the Booster the beam is divided into bunches that are in correct phase with the $84 \mathrm{RF}$ buckets.

After this stage, protons are transferred to the main injector[20] - a larger synchrotron that accelerates the beam to energies of $120-150 \mathrm{GeV}$. The $120 \mathrm{GeV}$ beam is used for fixed target experiments and also for antiproton production, while the 150 
$\mathrm{GeV}$ beam is injected into the Tevatron collider, where the proton and antiproton beams are accelerated to $980 \mathrm{GeV}$. Collisions are induced at two points on the ring where the CDF and D0 experiments have set up their detectors.

\subsubsection{Antiprotons}

Antiprotons are created by bombarding a nickel target with a high intensity 120 $\mathrm{GeV}$ proton beam. The proton beam is diverted from the main injector and focused by quadropole magnets onto the target. Just before collision, the RF fields are lowered, so that the bunched beam diffuses to a longer bunch length. The RF fields are then raised and the beam becomes bunched again. For a short period of time, the bunch length is shorter than before the RF manipulations began, but eventually the bunch length reaches the previous "equilibrium" length - the goal is to have the shortest bunch length at the exact time that the beam collides with the nickel target, so that the time-spread for the resulting secondary particles is smallest. This increases the efficiency of antiproton collection. The aforementioned process is called a "bunch rotation" - although it looks like "bunch compression" is a more appropriate name, the term rotation applies because the bunch gets rotated in the previously described phase space, decreasing the time spread of the bunch but increasing the momentum spread. After the collision, a spray of secondary particles results, traveling mostly at small angles with respect to the incident proton beam. A lithium magnetic lens is used to focus the resulting spray, and magnets are used to collect and divert negatively charged particles that have momenta near $8 \mathrm{GeV} / c$, while the rest (ignominiously) crash into the beam dump.

The $8 \mathrm{GeV} / c$ antiprotons are fed into a machine called the Debuncher[20], which is a triangular synchrotron with rounded edges depicted in Figure 2.6. In the Debuncher, the antiprotons are sufficiently energetic such that their speed does not change much with small changes in energy. However, particles of higher momenta bend less in the magnetic fields, and take a longer path around the accelerator and arrive at the RF station a little later on the next lap. As a result, the higher-energy 


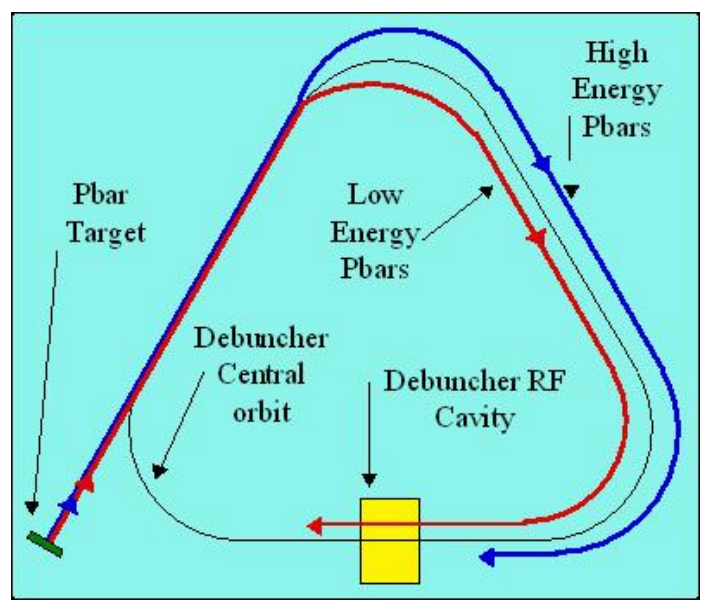

Figure 2.6: A schematic representation of the Debuncher.

particles experience a lower RF field than the rest of the particles. Lower energy particles, on the other hand, take a shorter lap around the debuncher and arrive earlier to the RF station, and thus experience a slightly higher RF field. The effect is a net force that shepherds the antiprotons to $8 \mathrm{GeV}$. As the momentum-spread shrinks, the time-spread increases (Lioville's theorem) and this the beam is "debunched". The beam is also cooled in the Debuncher. Cooling refers to reducing the transverse momentum spread of the particles in the beam. The method used in the Debuncher is called stochastic cooling. The beam is monitored by extremely sensitive pickups, which are cooled to near $4.6 \mathrm{~K}$ to reduce thermal noise. A signal is picked up that corresponds to the beam's momentum in a transverse direction. This signal is amplified and applied to the beam at a point downstream at the precise time to counteract the beam in that direction. This scheme is shown in Figure 2.7.

The antiprotons are transferred from the Debuncher to the Accumulator[20], which is another triangular shaped synchrotron. In the Accumulator, the beam is decelerated to $7.85 \mathrm{GeV}$, and undergoes stochastic cooling in both the transverse and the horizontal directions. The beam accumulates there until it is rebunched into 4 bunches by slowly increasing the RF fields, and transferred into the Main Injector or the Recycler. Once the antiprotons are in the Main Injector, they follow 


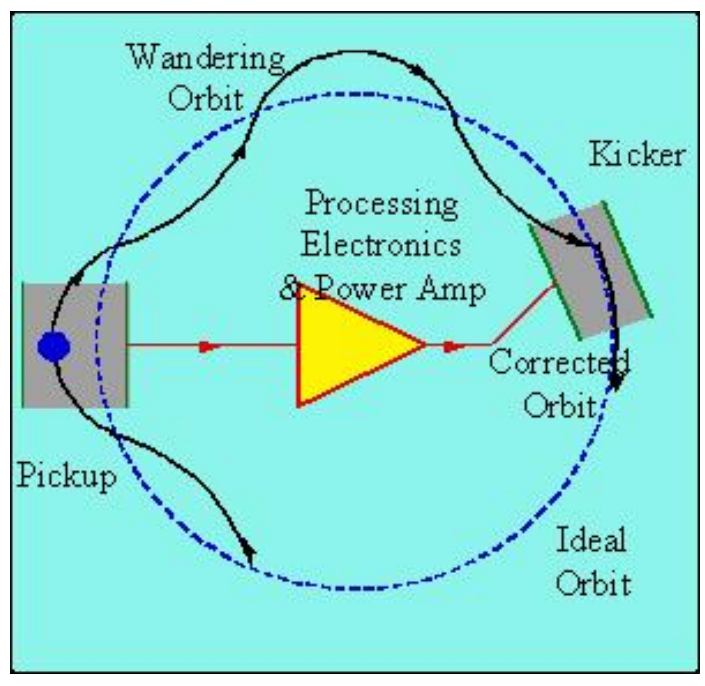

Figure 2.7: A diagram depicting the stochastic cooling process.

the same path (in the opposite rotational sense) to collisions as described for the protons. The process of producing and storing antiprotons is called "stacking". The stacking rate decreases as the Accumulator becomes full. To avoid this, the beam in the Accumulator is occasionally transferred to another storage ring called the Recycler. The Recycler is a synchrotron housed in the same tunnel as the Main Injector. Since the Recycler uses permanent magnets, it is a relatively safe and economical way to store antiprotons and to keep the stacking rate high. The beam can also be cooled here in a process called Electron Cooling - an electron beam with very small transverse momentum-spread runs parallel to the antiproton beam, and heat (the random transverse momenta of the beam particles) is exchanged from the antiprotons to the electrons. The cooled antiprotons are injected to the Main Injector, where they are accelerated to $150 \mathrm{GeV}$ and in turn injected into the Tevatron.

\subsubsection{Tevatron Performance}

The Tevatron collides 36 proton and 36 antiproton bunches at a CM energy of 1.96 TeV with 396 ns beam crossings. This can be compared with the first run of the 
Collider Run II Integrated Luminosity

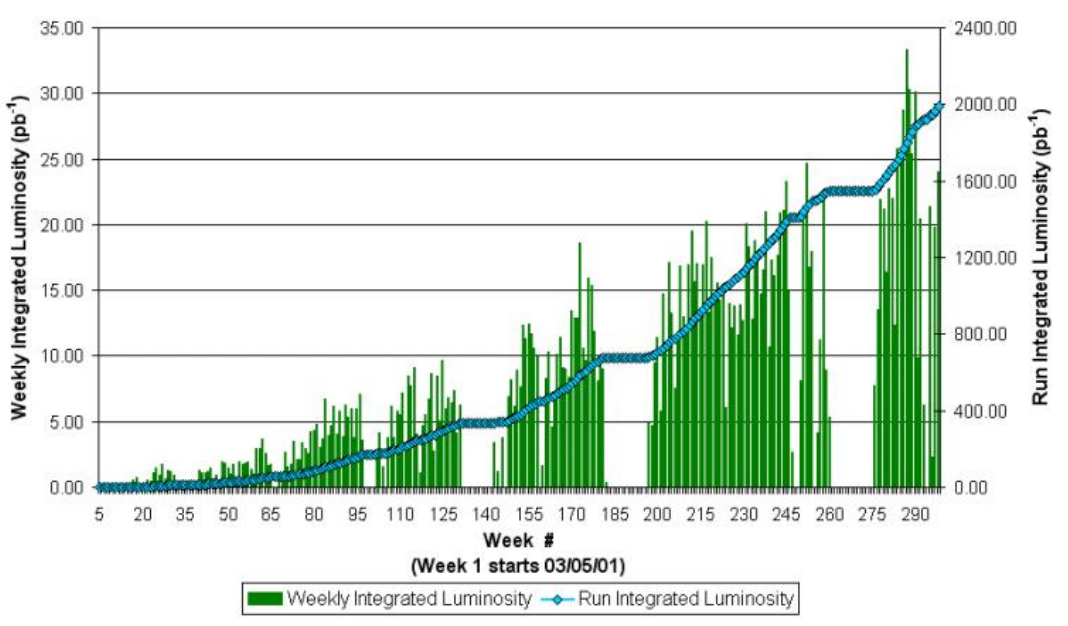

Figure 2.8: The Tevatron delivered almost $2 \mathrm{fb}^{-1}$ per experiment, and has recently achieved $30 \mathrm{pb}^{-1}$ per week[21].

Tevatron, where 6 proton and 6 antiproton bunches were collided at $1.8 \mathrm{TeV} \mathrm{CM}$ energy and 3500 ns intervals. The total luminosity recorded by CDF for Run I was $106 \mathrm{pb}^{-1}$. Presently, CDF is recording data at a rate of $\sim 106 \mathrm{pb}^{-1}$ per month. In September 2006, the Tevatron set a new world record for hadron colliders with an initial luminosity of $2.28 \times 10^{32} \mathrm{~cm}^{-2} \mathrm{~s}^{-1}$. Notably, the entire antiproton beam was transferred from the Recycler to the Main Injector. The use of the Recycler has helped increase the stacking rate of antiprotons, which in turn increases the luminosity. The delivered integrated luminosities by the Tevatron are plotted in Figure 2.8.

\subsection{CDF Detector}

The Collider Detector at Fermilab (CDF), shown in Figure 2.9 is a conglomerate of detectors designed to characterize myriad outcomes of $p \bar{p}$ collisions at high energies and interaction rates. The detector consists of a solenoidal magnetic spectrometer surrounded by calorimetry systems and outer muon chambers. The radial devel- 
opment of the detector lends itself to particle identification as described in Figure 2.10. The CDF detector has, but for minor exceptions, forward-backward symmetry about the nominal interaction point and azimuthal symmetry about the beam line. The official CDF coordinate system is right-handed with the $z$-direction taken as the direction of the incoming protons and the $y$-direction pointing vertically upwards. The azimuthal angle $\phi$ starts at 0 along the positive $x$-direction, and increases towards positive $y$. The polar angle $\theta$ is zero along the positive $z$-axis, and increases along the negative $z$-axis. A substitution is made for the variable $\theta$, and we prefer to use the relativistically invariant pseudorapidity $(\eta)$ defined as

$$
\eta=-\ln [\tan (\theta / 2)]
$$

Some advantages of using $\eta$ over $\theta$ are that particle production at hadronic colliders are roughly constant in $\eta$, and that Lorentz invariance implies the angle $\delta \eta$ between two particles is the same for all frames that vary only in $z$-component of velocity. This is especially useful because the CM frame at hadron colliders has some unknown $z$-velocity. Table 2.1 lists the correspondence between $\theta$ (in degrees) and eta. At hadron colliders, most of the interactions result in particles that are only slightly deflected from the beam direction. This means that in most beam crossings there are several particle with energies at a significant fraction of $980 \mathrm{GeV}$ in the far "forward/backward" ${ }^{5}(|\eta|>3.6)$ region. Much of the interesting physics involves substantial momentum transfer and results in secondary particles in the region $|\eta|<$ 2 , and it is in this region where CDF is most sensitive. CDF has undergone a series of upgrades to ready it for the high occupancy and rates associated with the high luminosity running of the Tevatron.

\footnotetext{
${ }^{5}$ The region $|\eta|<1$ is known as "central". Regions with $\eta>1$ are known as "forward", and $\eta<-1$ are referred to as "backward".
} 


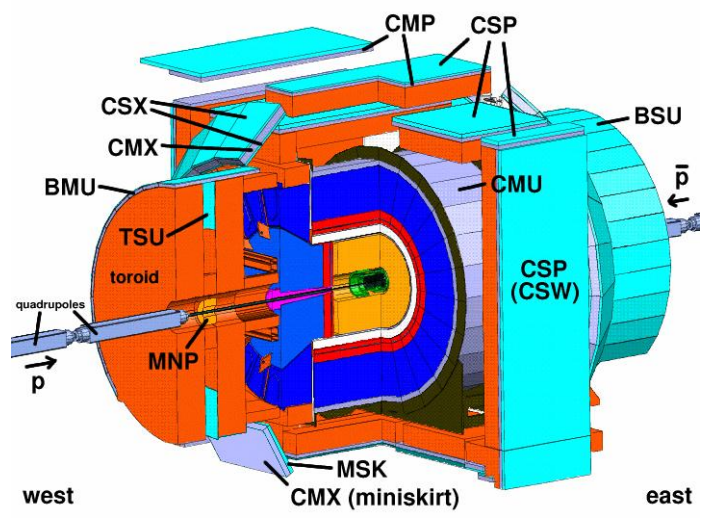

(a)

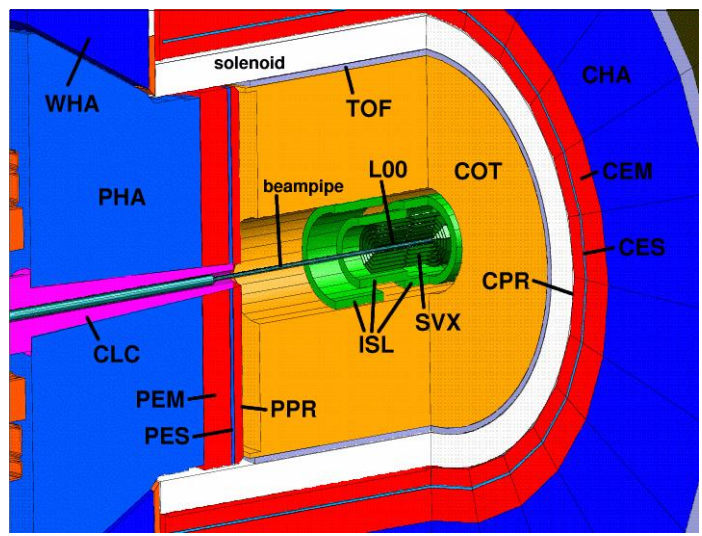

(b)

Figure 2.9: (a) An external view of the CDF with only the muon systems labeled. (b) An internal cutaway of the CDF. Note that the forward detectors (PHA, CLC, PES) are cut and peeled away $90^{\circ}$ from their rightful location. The acronyms will be explained below.

\begin{tabular}{|c|c|c|c|c|c|c|c|}
\hline$\theta$ & 0 & 10 & 20 & 30 & 45 & 60 & 90 \\
\hline$\eta$ & $\infty$ & 2.44 & 1.74 & 1.31 & 0.88 & 0.55 & 0.0 \\
\hline
\end{tabular}

Table 2.1: Theta (in degrees) and corresponding values of $\eta$.

\subsubsection{The Silicon Tracking Detectors}

\section{Semiconductor Detectors}

A silicon microstrip detector is basically an array of reverse-biased diodes cou- 


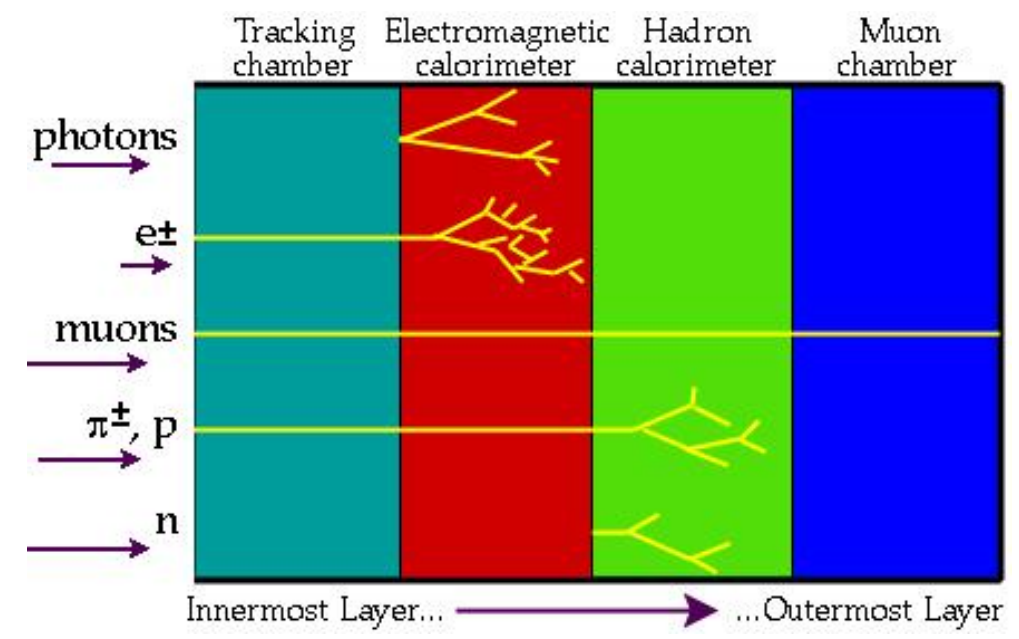

Figure 2.10: Particles are identified according to how they behave as they traverse successive detector components. All charged particles will leave a track in the tracking chamber (teal) - their charges and momenta can be measured from the tracks. Electrons and photons will deposit practically all of their energies in the electromagnetic calorimeter (red). Hadrons will leave energy in the electromagnetic calorimeter, but will deposit most of their energy in the hadronic calorimeters (green). Most of the time, the only particles that will make it past all the aforementioned detectors are muons. These leave tracks in the muon drift chambers (blue). Neutrinos, of course, leave no trace in any detector component, and show up as a net imbalance in the transverse energy deposition.

pled to charge integrators and other readout electronics. Typically, the "bulk" of the sensor is doped to one type ( $\mathrm{p}$ or $\mathrm{n})$, while the readout strips are doped the opposite way. For silicon detectors, the impurities are more concentrated at the far ends of the diode, while most of the bulk is nearly intrinsic (undoped)- making for a p-i-n "junction" between the strips and the bulk. If a reverse-bias voltage is applied, the depletion zone widens and covers the entire "i" region. Ionization by charged particles creates electron-hole pairs in the depletion zone. Before they can recombine, electrons drift to the anode and the holes to the cathode. The time scale for this process is small compared to that of the power supplies, thus the current causes a momentary drop in bias voltage which can be picked up by capacitively coupled amplifiers. The integrated voltage spike is proportional to the energy lost by the ionizing particle. The semiconductor detector's performance depends on many 
design and environmental conditions. Most charged particles will create enough electron/hole pairs to leave a signal, however, there is also noise due to thermal leakage currents through the p-n junction, and also environmantal pickup. The signal to (thermal)noise ratio $(\mathrm{S} / \mathrm{N})$ is usually pretty high (i.e. 10:1), but may degrade with increasing temperature and also with radiation damage to the sensors. The sensors themselves have some capacitance (a few $\mathrm{pF}$ ), and are thus sensitive to command signals coming through nearby cables. To lower the potential for such effects, care must be taken to properly ground the sensors, and the nearby electronics must be made as "quiet" as possible (i.e. by using Gray code, differential signals, and shielded or twisted-pair cables, and fiber optic readout when possible.).

The basic unit of the silicon detector at CDF is the "ladder". The name comes from the shape of the detector element, shown in Figure 2.11. Each ladder is composed of six silicon sensors with microstrips on each side ${ }^{6}$. One side has strips arranged in parallel to the beam direction and can detect the $\phi$ coordinate of charged particles that pass through the strips - this side is referred to as the axial or $r$ - $\phi$ side. The opposite side has strips with either a $1.2^{\circ}$ or $90^{\circ}$ angle with respect to the axial strips. The offset enables reconstruction of the $z$-coordinate of the charged particles that traverse the strips - this side is referred to as the $r-z$ or stereo side. The sensors are wirebonded to each other and also at each end to a "hybrid" unit which contains the SVX chips that collect, buffer, and digitize the charge collected on the sensors. The ladders have, depending on positioning within the silicon detector, between 8 and 28 chips on them, each reading out 128 channels.

The SVX3 chip shares the characteristics of two separately dedicated chips. The analog front end of the chip can integrate and write charge to a capacitor "pipeline" whilst the back end digitizes signals and sends the output to the rest of the data acquisition (DAQ) system. In this fashion the chip operates continuously without incurring any deadtime associated with signal conversion or readout. The analog front end has 128 channels, each with an amplifier, an integrator and a 46 cell

\footnotetext{
${ }^{6}$ With the exception of the innermost layer, which is single-sided.
} 
capacitor storage pipeline, along with some logic that handles pipeline control (e.g., writing/overwriting capacitor cells). The silicon strips are AC-coupled to front-end preamplifiers, and the charge is integrated with a 10-90\% rise time of $60 \mathrm{~ns}$. The difference in charge on the integrator before and after an event is transferred to cells in a 42-cell pipeline (4 cells are used as buffers for data awaiting readout to the back end), and the total charge in the integrator itself is reset at Tevatron "abort gaps" where there are a few successive buckets without beam. Pipeline cells are overwritten unless a trigger decision ${ }^{7}$ causes them to be tagged for digitization and readout. The digital back end of the chip contains an array of 128 comparators, a voltage ramp, and a counter used in digitization.

One important feature of the chip is called "Dynamic Pedestal Subtraction". In this mode, the counters of the analog-to-digital converters (ADC's) do not start until the comparators corresponding to the first 30 channels fire. This effectively rescales the average level of ADC counts for the channels (the "pedestal") on a given chip to zero, and any excess corresponds to thermal noise or actual signal. Any noise source common to all channels on a chip effectively gets rescaled to zero. This is important because it eliminates occupancy due to "pickup" of potentially strong control signals elsewhere in the detector, and makes threshold decisions easier during readout. The average minimally ionizing particle (MIP) deposits some 22,000 electrons in the silicon. This corresponds, at the typical 700 electrons per ADC count, to a signal of 31 ADC counts. This is to be compared with 3 ADC counts as the typical thermal noise, and the 256 count dynamic range of the ADC.

There are over $700 \mathrm{k}$ channels in the silicon detectors. At certain stages in DAQ, the readout of these channels is serialized. To avoid congestion at higher levels of DAQ, it is necessary to read out as few channels as necessary. The SVX3 chip is able to run in two "sparse" modes. In one mode, only channels with signals above an adjustable threshold are read out, and in another mode the adjacent channels are

\footnotetext{
${ }^{7}$ The trigger system is described later. Dedicated trigger hardware located in crates near the detector decide whether to keep or reject and event, and the decision is forwarded to all subdetectors.
} 
also read out. The latter mode facilitates "clustering" of signals to achieve spacial resolution even finer than the $\sim 60 \mu \mathrm{m}$ pitch (the distance between two adjacent strips) of the detector. After signals are digitized, chips from several ladders send their data to a portcard. Portcards relay commands from the main Silicon controller, and power from external supplies, to the ladders. Portcards also convert the readout information from the individual ladders to a serialized optical signal, and send it out of the collision hall to higher stages of DAQ.

\section{The SVX Detector Family}

The innermost tracking chambers in CDF are silicon microstrip detectors. There are three distinct silicon detector systems comprising 7 or 8 concentric cylindrical layers of silicon sensors covering regions up to $|\eta|=1.9$. At the smallest radius, mounted on the beampipe, is a single-sided silicon strip detector called Layer 00 (L00). The L00, because of its small radius, can improve impact parameter resolution and secondary vertex reconstruction. Some special design features are required for successful implementation of silicon detectors at such small radii. Firstly, the sensors for L00 are able to hold bias voltages of 700-1000 V, or roughly 10 times the amount used on the rest of the silicon detectors. This ensures that the sensors can be reverse biased and depleted even after degradation occurs from the high radiation doses associated with the small radii. Secondly, the readout electronics for the L00 are placed at larger $z$ and larger radii than the sensors, making for less material in the active tracking volume, and also lowering the radiation dose to the readout electronics.

Between 2.44 and $10.6 \mathrm{~cm}$ in radius the SVX II detector (replacing the original Silicon Vertex Detector (SVX) of Run I) has five layers of double-sided silicon arranged in 12 wedges of $15^{\circ}$. The 12 wedges make up a "barrel" - so called due to its cylindrical shape, and there are three barrels in the SVX, each one covering $29 \mathrm{~cm}$ in the $z$-direction. The combined detector is placed symmetrically about the nominal interaction point. The SVX detectors are double-sided silicon, with Layers 
0,2 , and 4 having $90^{\circ}$ stereo strips, while Layers 1 and 3 have $1.2^{\circ}$ "small angle stereo" strips. The readout pitch for the $r$ - $\phi$ and small angle stereo strips is $60 \mu \mathrm{m}$, while the $90^{\circ}$ strips have a $141 \mu \mathrm{m}$ pitch.

At larger radii than the SVX, there is a detector called the intermediate silicon layer (ISL). The ISL is also structured in three barrels of twelve wedges each. The central barrel has one layer of silicon at a radius of $22 \mathrm{~cm}$, while the barrels at large $|z|$ have two layers at 20 and $28 \mathrm{~cm}$. The ISL detector measures $175 \mathrm{~cm}$ from end to end, and covers the pseudorapidity region $|\eta|<1.9$. An $\mathrm{r}-z$ view of the detector is shown in Figure 2.13. The ISL can help link tracks that are reconstructed in the SVX to tracks in the drift chamber. Since the drift chamber covers only the central region, the two layers of ISL provide important spacepoint measurements at large radii for forward/backward tracks. Tracking algorithms that use ISL information as well as SVX information typically have signal to noise ratios that are three times higher than those that use SVX info alone. The impact parameter and $p_{T}$ resolution also improve when ISL information is used. The combined ISL+SVX tracking has $p_{T}$

resolution $\delta p_{T} / p_{T}^{2} \leq 0.004(\mathrm{GeV} / c)^{-1}$, impact parameter resolution $\delta d_{0}=15 \mu \mathrm{m}$, and a $\phi_{0}$ resolution of 0.3 mrad.

The silicon detectors allow reconstruction of secondary vertices associated with decays of $B$ mesons. The resolution for displaced vertices is about $30 \mu m$ in the radial direction and $60 \mu m$ in the $z$-direction. This will facilitate a wide spectrum of physics ranging from Bottom and Top quark measurements and even Higgs searches. A cross section of the SVXII tracking system is shown in Figure 2.12.

\subsubsection{The Central Outer Tracker}

The Central Outer Tracker (COT) is a large drift chamber used to track charged particles in the region $|\eta|<1$ and between radii of 40 and $137 \mathrm{~cm}$. It was designed to reconstruct tracks at the high luminosities and interaction rates associated with the second running of the Tevatron (Run II). The maximum drift times for the COT can be as little as 100 ns using a 50:35:15 Ar-Et- $\mathrm{CF}_{4}$ gas mixture, and occupancy 


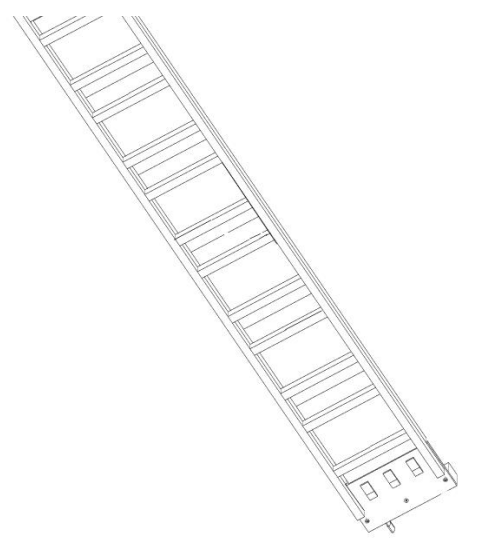

Figure 2.11: A depiction of an ISL Ladder. The support structure resembles a ladder, thus the name. Three silicon sensors are fixed to the support structure. The microstrips are wirebonded to each other, and at each end they are bonded to a "hybrid" containing DAQ hardware.

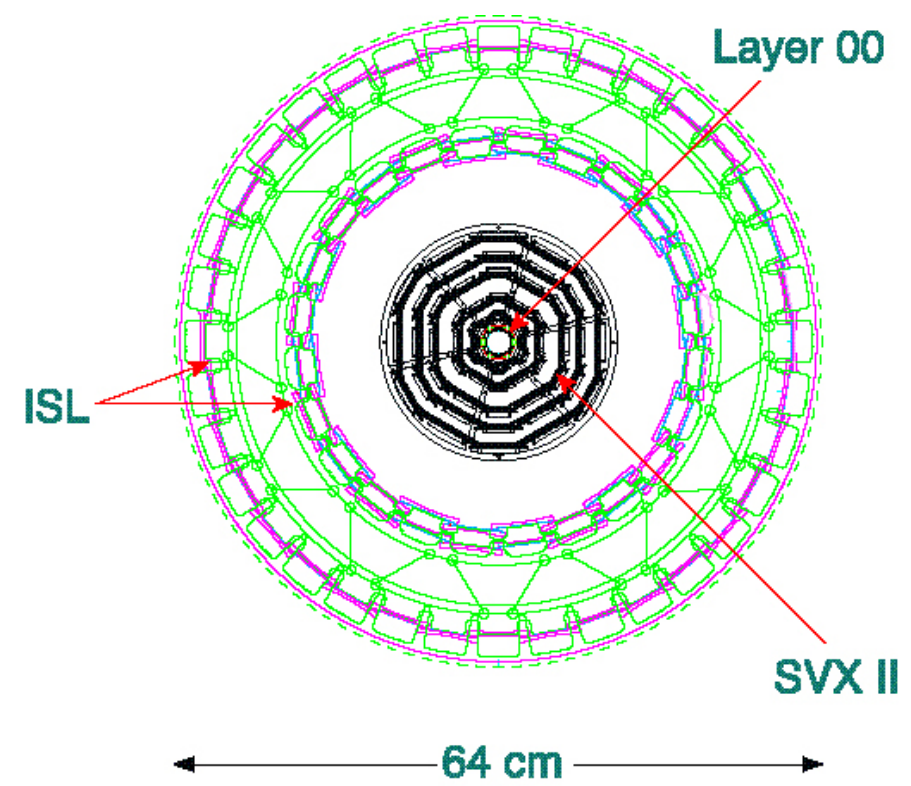

Figure 2.12: A cross section of the SVXII detector.

is not an issue with the $396 \mathrm{~ns}$ bunch spacing at the Tevatron. The COT consists of 8 superlayers (SL's) composed of a variable number of self-contained cells ${ }^{8}$ that

\footnotetext{
${ }^{8}$ The number increases from 168 for SL1 to 480 at SL8 to maintain the same cell (and wire) density at increasing radii.
} 


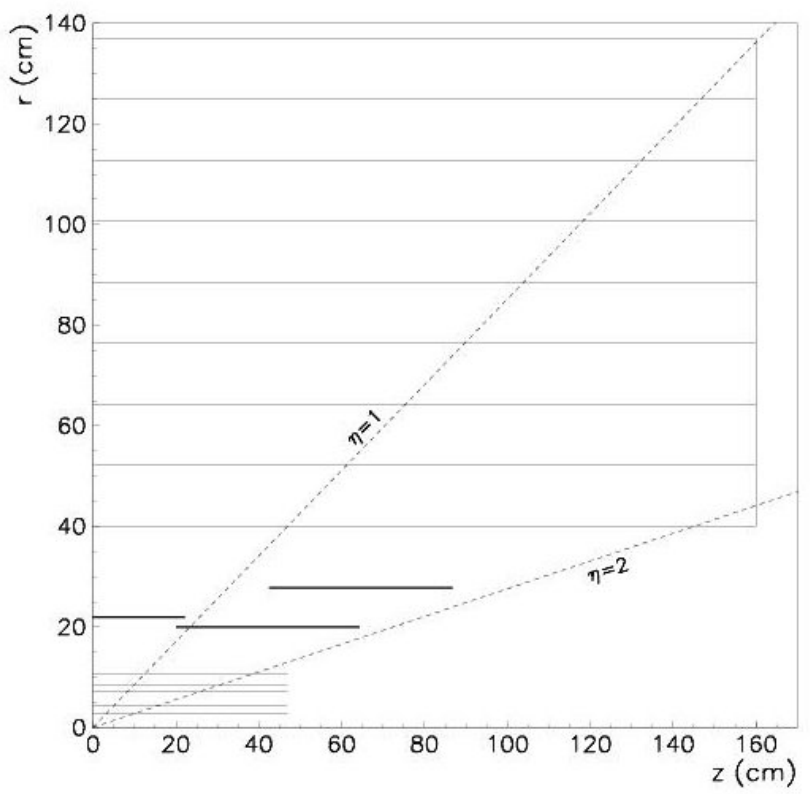

Figure 2.13: An $r-z$ cross section of the ISL and SVXII detectors. Tracks in the region $|\eta|<1.9$ will through pass 7 or 8 layers of silicon.

are at $35^{\circ}$ angles with respect to radial lines from the $z$-axis ${ }^{9}$. Each cell contains 12 positively biased sense wires, and 13 positively biased potential wires ${ }^{10}$ made of 1.6 mil gold-plated tungsten that run $310 \mathrm{~cm}$ in the $z$-direction across the COT. The edges at the inner and outer radii of the cells are insulating mylar strips that also include two field shaping wires. The two azimuthal edges have field panels with gold-plated mylar cathodes. The radial and azimuthal panels encase the cell both physically and electrostatically. Figure 2.14 shows a cell from SL2, while Figure 2.15 shows the equipotential lines in a typical cell. Even numbered superlayers have wires that are parallel to the $z$-axis, and give $r$ - $\phi$ tracking resolution, while the

\footnotetext{
${ }^{9}$ The "Lorentz Angle" is the angle at which a charged particle will drift with respect to the electric field in the magnetic spectrometer, and depends on the strengths of the electric and magnetic fields. If we consider that a particle moves at a fairly constant velocity (until it gets within a few radii of the sense wire) then the Lorentz Force Law reads $F=q(E \hat{x}+v B \hat{y})$ where $\hat{x}$ and $\hat{y}$ are directions of the electric and magnetic forces respectively, and $v$ is the drift velocity of the electrons liberated by the charged particle that is being tracked.

${ }^{10}$ The sense wires have a surface field of $180 \mathrm{kV} / \mathrm{cm}$, while the potential wires have a surface field of $118 \mathrm{kV} / \mathrm{cm}$. Although the potential wires are positively biased, they collect only a negligible amount of electrons because, the gain changes by a factor of two for every $6 \mathrm{kV} / \mathrm{cm}$ change in surface field.
} 
odd-numbered "stereo" layers are angles at $\pm 3^{\circ}$ and enable measurements of the $r-z$ trajectories of the tracks. Because of the large number of stereo wires, the COT has good $r-z$ resolution and performs well in high luminosity environments where several interactions may occur in one bunch crossing. Track reconstruction algorithms that rely solely on the COT are able to reconstruct tracks with transverse momenta $p_{T}>400 \mathrm{MeV}$ with a $p_{T}$ resolution of $\delta p_{T} / p_{T}^{2} \leq 0.002(\mathrm{GeV} / c)^{-1}$, an impact parameter resolution of $600 \mu \mathrm{m}$, and a vertex $z$ resolution of $5 \mathrm{~mm}$. The COT is also used to measure $d E / d x$ for charged particles. The amount of charge collected by a wire is related to the time that it takes to collect the charge $(\Delta t \approx \log (Q))$. The $d E / d x$ is obtained by measuring the time for which the signal on the sense wire is over threshold. All 96 layers of wires in the COT have $d E / d x$ measurement implemented, compared with just 54 layers in the drift chamber used in Run I. Overall $d E / d x$ measurement resolution is better than $10 \%$. A mass limit of 134 $\mathrm{GeV} / c^{2}$ on a quasi-stable $\mathrm{H}^{++}$has been achieved by $d E / d x$ measurements using the COT as well as the calorimeters[22].

\subsubsection{The Time Of Flight Detector}

Housed between the COT and the solenoid, the Time of Flight (TOF) detector is a cylindrical array of 216 scintillators, each measuring $4 \mathrm{~cm} \mathrm{x} 4 \mathrm{~cm} \times 280 \mathrm{~cm}$. At a radius of $1.4 \mathrm{~m}$, it takes a particle traveling at the speed of light about $5 \mathrm{~ns}$ to reach the TOF. The light from the scintillators is collected and amplified by PMT's, digitized, and merged with the event data. The resolution for the time of flight is about 100 ps. This helps discriminate between $\pi^{ \pm}$'s and $K^{ \pm}$'s at low $p_{T}$, and can also be used, along with $d E / d x$ measurements, to search for stable heavy charged particles. The TOF is also used to identify and remove of cosmic ray muons. High $p_{T}$ muons resulting from beam collisions start at the interaction point and travel similar distances at similar speeds before leaving a signal in the TOF. The difference in the TOF hits of the two muons is typically less than 2 ns. On the other hand, a cosmic ray with a small impact parameter must travel at least 2.8 meters between 


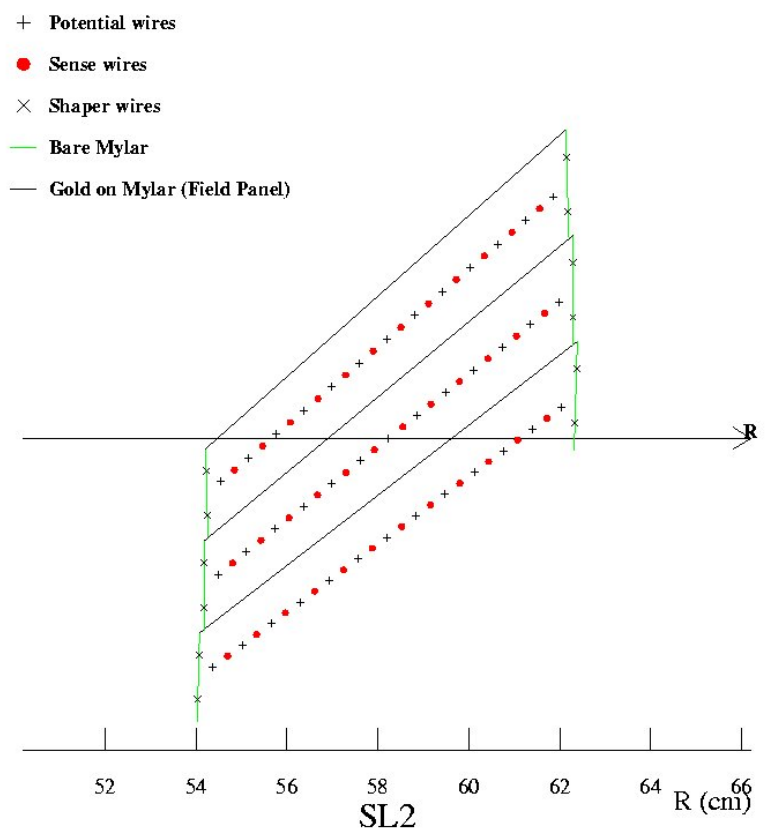

Figure 2.14: Cell layout for SL2. The radius (in CDF coordinates) increases from left to right on the page, and is plotted in $\mathrm{cm}$ units.

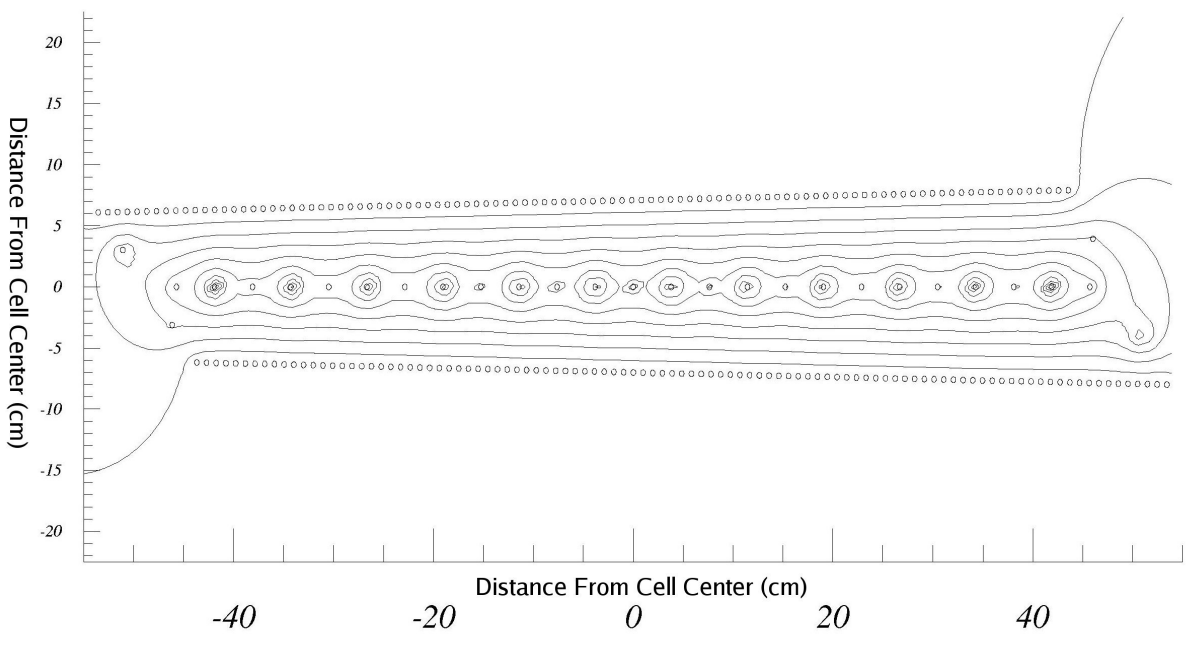

Figure 2.15: Equipotential lines for a typical COT cell.

its first and second interaction with the TOF, thus the two TOF hits are typically at least $10 \mathrm{~ns}$ apart. We use a simple cut on the time-difference of the TOF hits for two muons in an event to remove most cosmic ray muons while retaining nearly 
$100 \%$ of the muons resulting from $p \bar{p}$ collisions[23].

\subsubsection{The Calorimeters}

The calorimeters are designed to measure the energies deposited by particles as they pass through, and also to fully or partially absorb certain classes of particles. We briefly discuss some important modes of energy-loss in material by particles in the $\mathrm{GeV}$ energy range before describing the CDF calorimeters. High energy $(\geq$ $10 \mathrm{GeV}$ ) photons primarily lose energy through pair production in the electric fields of nuclei in the absorbing material. Electrons in the $\mathrm{GeV}$ range lose energy primarily through the radiation of photons, called Bremsstrahlung (breaking radiation). As high energy photons or electrons pass through the calorimeter, they lose energy through a cycle of Bremsstrahlung and pair production. For example, a photon produces an electron-positron pair, each radiating photons, which in turn produce pairs, and so on. The number of particles grows exponentially, and the particles created become less and less energetic at each juncture (for example, each particle in an electron-positron pair has on average half the energy as the "mother" photon). Eventually, particles are not energetic enough to beget newer particles, and lose energy through ionization until they are absorbed by the material. The process described above is known as electromagnetic showering. It is useful to define a quantity called the radiation length, $X_{0}$ as the average distance a particle must travel in order for just $1 / e$ of its original energy ro remain.

$$
E(x)=E_{0} e^{-x / X_{0}}
$$

The electromagnetic shower grows to its maximal profile at 4-7 $X_{0}$, then shrinks as the fraction of particles in the shower that are energetic enough to produce pairs decreases. The energy of the incident electron is proportional to the number of particles at the maximal profile of the shower.

Energetic hadrons lose energy primarily through inelastic nuclear interactions. 
Secondary particles produced by nuclear interactions subsequently undergo inelastic collisions of their own. There is usually a fast component due to the electromagnetic showers of tertiary photons that result from decays of secondary $\pi^{0} \mathrm{~s}$, and a slower component due to the hadronic aspect. The cross section for such reactions is much smaller than those involved in electromagnetic showers. As a result, the nuclear interaction length, $\lambda_{I}$, analogous to $X_{0}$, is much longer, and hadronic calorimeters need to be much larger to contain the expansive "hadronic cascades" ${ }^{11}$. Leptons do not partake in the strong interaction, and so muons, as with electrons, lose energy by ionization or Bremsstrahlung in the calorimeters. The mean energy loss for Bremsstrahlung is proportional to the energy of the particle and inversely proportional to the square of its mass. Therefore, the radiative energy-loss of a muon is four orders of magnitude less than that of an equally energetic electron. Bremsstrahlung becomes a significant mode of energy-loss for muons only above energies of 100 $\mathrm{GeV}$. Muons produced at the Tevatron mainly lose energy by ionization and as a result deposit very little energy (a few $\mathrm{GeV}$ at most) in the detector. Neutrinos, of course, leave no trace in the detector at all, but can be inferred from an imbalance in the total transverse energy. Other sources of energy imbalances (jet mismeasurements, particles going through cracks, and even other neutrinos) can cause large uncertainties in the "missing transverse energy."

Sampling calorimeters are "sandwiches" of some absorber, such as lead or iron, and active layers of scintillator. Electromagnetic showers and hadronic cascades occur mostly in the absorber layers, and the charged component leaves a signal in the scintillators. The light is collected by PMT's, and the total energy, being proportional to the number of charged particles produced in the shower or cascade, is proportional to the PMT output.

CDF has nearly $4 \pi$ solid angle calorimeter coverage. The central electromagnetic and hadronic calorimeters cover the region $|\eta|<1.1$, while the plug electromagnetic and hadronic calorimeters cover the region $1.1<|\eta|<3.4$. The central calorime-

\footnotetext{
${ }^{11}$ For comparison, in lead $\lambda_{I}$ is $17 \mathrm{~cm}$ while $X_{0}$ is $6 \mathrm{~mm}$.
} 
ter is composed of 24 wedges, each covering $15^{\circ}$ in azimuth. Each wedge has 20 segments, called towers, that cover roughly 0.11 in $\eta$ and point to the nominal interaction region. The inner ${ }^{12}$ ends of the towers compose the central electromagnetic calorimeter (CEM) while the outer parts are the central hadronic calorimeter (CHA). As mentioned earlier, electrons and photons deposit almost all their energy in the CEM, and do not significantly penetrate the CHA. The CEM is a 31 layer lead-scintillator sandwich, corresponding to $19 X_{0}$. The energy resolution is $\sigma(E) \leq 0.135 \times \sqrt{E} \oplus 0.017 \times E$. The second term is the average uncertainty for individual tower calibrations, while most of the first term comes from the limited total thickness of lead used for the calorimeter. There is a slow loss of light yield in the scintillators, which causes very slow degradation of the detector's performance. As a result, the stochastic error has increased from $0.135 \times \sqrt{E}$ to $0.14 \times \sqrt{E}$ during the 21-year existence of the CEM.

The calorimeter was initially calibrated with testbeams of electrons and $\pi^{+}[24$, 25], but is continually calibrated with radioactive sources as well as xenon and LED light flashers connected to the PMT's. Furthermore, measurements of $\Upsilon \rightarrow e e$, $Z \rightarrow e e$ and $W \rightarrow e \nu_{e}$ events can reduce variations in the overall electromagnetic (EM) scale, and the mean of the energy to momentum distribution for pure electron samples can result in tower-to-tower and even intra-tower corrections to the energy response.

The CEM has an embedded multi-wire proportional chamber (MWPC) at $6 X_{0}$ the depth where typical electromagnetic showers have their maximal profile. For this reason it is called the Central Shower Max detector (CSM). The chamber consists of a layer of 2-mil gold-coated wires running along the $z$-axis, housed in a gas-filled chamber. The inner side of the chamber is segmented into cathode strips that are perpendicular to the wires, enabling 3-D spacepoint measurements on developing showers. The electric field inside the chamber is small and constant, except near the anode wires, where it takes on a $1 / r$ form. Electrons that result from ionization of

\footnotetext{
${ }^{12}$ With respect to the interaction point.
} 
the gas cause an avalanche in the high fields present within a few radii of the wire, while the positive ions drift slowly to the cathode and leave a signal on the strips. The wires and strips are $1.4 \mathrm{~cm}$ and $2 \mathrm{~cm}$ apart, respectively. Clustering of charge on multiple wires and strips enables finer spacial resolution through interpolation than the pitch of the wires or strips alone. The CSM is useful for matching tracks tightly to EM clusters, and also for rejecting backgrounds to electrons, such as overlapping $\pi^{ \pm} \pi^{0}$ combinations or high EM fraction $\pi^{ \pm}$'s. Another MWPC called the central preradiator $(\mathrm{CPR})$ is positioned just before the CEM. The CPR is useful for counting the number of MIPS in a given area. This helps to distinguish between photons, $\pi^{0}$ 's, $\pi^{ \pm}$'s and electrons. Finally, tungsten absorbers and scintillator tiles are placed in the "cracks" between CEM wedges to catch particles that are in these otherwise inactive regions of the calorimeter ${ }^{13}$.

The CHA is composed of alternating layers of iron and scintillator, and is $4.7 \lambda_{I}$ thick. The energy resolution of the CHA is $\sigma(E) \leq 0.5 \times \sqrt{E}$. The CHA has been calibrated with test beams[24], and is continually calibrated with radioactive and light sources. Furthermore, the calorimeter and jet reconstruction algorithms are calibrated using isolated tracks, dijet, photon-jet, and $Z$-jet balancing. Coverage in the pseudorapidity region $0.9<|\eta|<1.3$ is provided by the "End Wall Hadron" calorimeter (WHA). The WHA has similar construction and segmentaion as the CHA[26].

In the forward/backward region, the plug electromagnetic and hadronic calorimeters have replaced the gas calorimeters which were used in Run I. The segmentation of the plug EM and HAD calorimeters (PEM and PHA) is the same. In the region $|\eta|<2.1$, the towers cover $7.5^{\circ}$ in phi, while in the more forward/backward regions, $15^{\circ}$ towers are used. The pseudorapidity segmentation retains the $\delta \eta=0.11$ structure as long as possible, but the last few towers cover progressively more $\Delta \eta$. Figure 2.16 shows an $r-z$ cross section of the plug calorimeters, while Figure 2.17 shows the segmentation within a wedge. The PEM is a lead/scintillator sampling

\footnotetext{
${ }^{13}$ The cracks between wedges add up to almost $7 \%$ of the total phi coverage.
} 


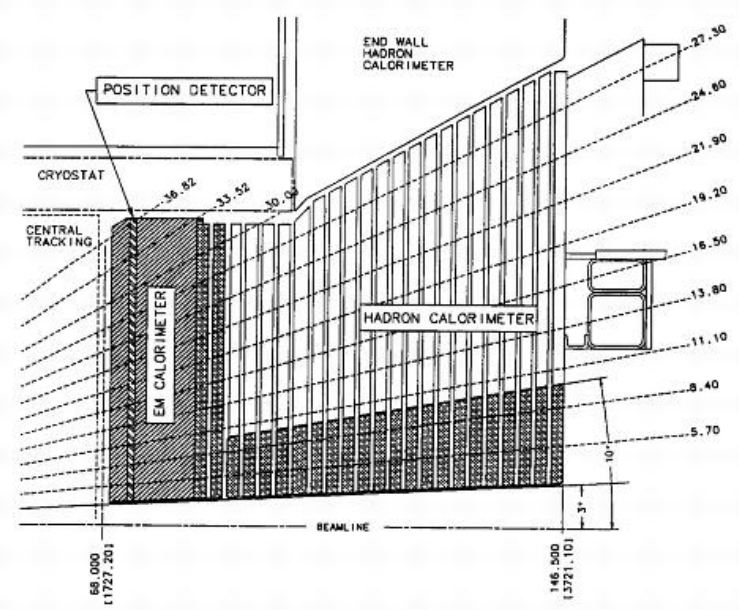

Figure 2.16: An $r-z$ cross section of the plug calorimeter.

calorimeter of $21 X_{0}$, and has a resolution $\sigma(E) \leq 0.16 \times \sqrt{E} \oplus 0.01 \times E$, where the second term is again caused by tower-to-tower variations in response. The first layer of scintillators can be read out separately from the rest of the PEM, and can be used as a preshower detector. The PEM also has a shower maximum detector called the plug shower max (PSM). Rather than using an MWPC, the PSM has two layers of $5 \mathrm{~cm}$ wide scintillating strips, arranged at angles of $\Delta \phi= \pm 22.5^{\circ}$ with respect to radial line associated with each wedge, as shown in Figure 2.18. The PSM enables 3D spacepoint measurement of developing showers with a $1 \mathrm{~cm}$ resolution. This helps in the reconstruction of forward/backward electron tracks with the spacepoint acting as an outer seed for tracking algorithms that otherwise have only information from silicon hits at their disposal. The PSM is calibrated using a ${ }^{137}$ Cs source, and strip-to-strip variations are within 10\%. The Plug Hadronic Calorimeter (PHA), is an iron/scintillator sandwich of $7 \lambda_{I}$, with a resolution of $\sigma(E) \leq 0.80 \times \sqrt{E} \oplus 0.05 \times E$. It is calibrated using wire-mounted radioactive sources $\left({ }^{137} \mathrm{Cs}\right)$, and also with physics data. As with the other calorimeters, the plug PMT's are calibrated with a light source weekly. 


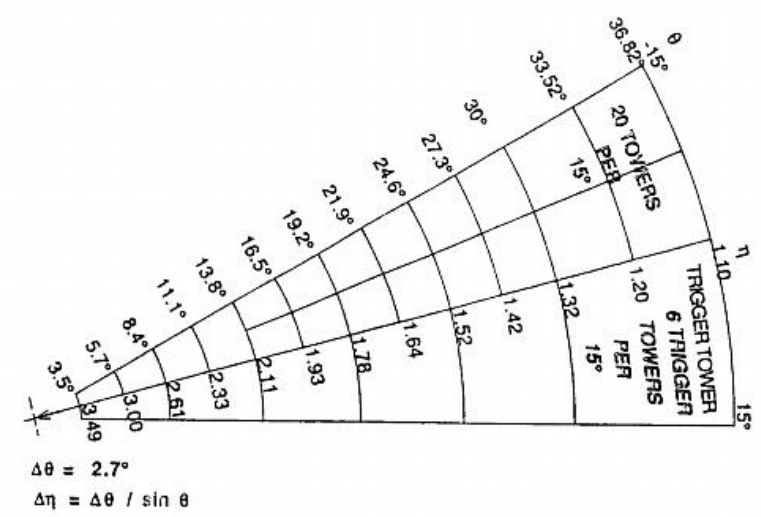

Figure 2.17: Two wedges of the plug are shown. At lower $|\eta|$, there are two towers per wedge. To save time at the trigger level, two to four calorimeter towers are read out as one trigger tower.

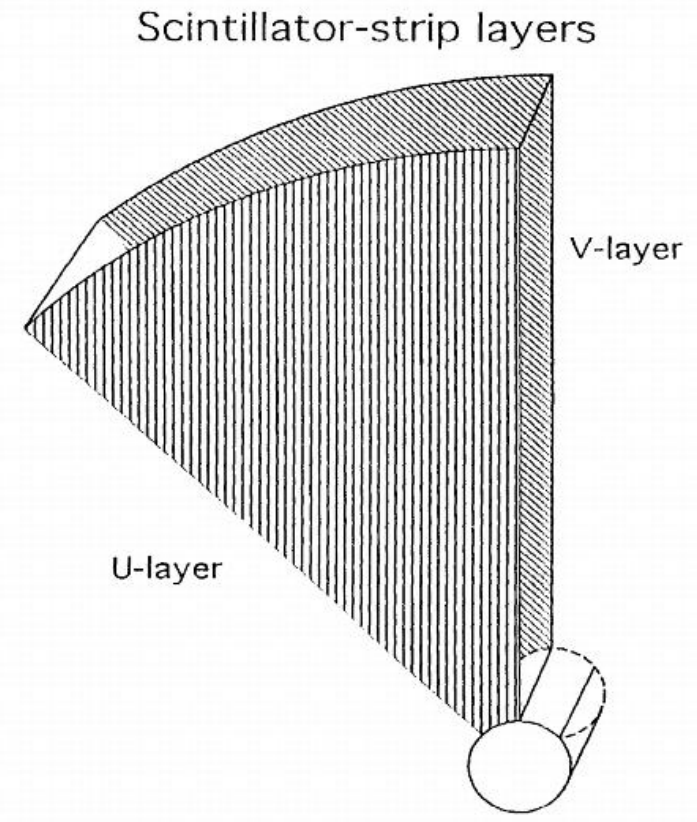

Figure 2.18: A $45^{\circ}$ sector of the PSM.

\subsubsection{The Muon System}

Muon detection at CDF has four components. The tracking system measures the muons' charge and momenta, the solenoid and calorimeters act as absorbers, outer 
wire chambers serve as muon detectors, and scintillators are used for triggering. The CDF muon system has four separate detectors, as summarized in Table 2.2. The muon detectors are all based on single-wire proportional chambers filled with a 50-50 Argon-Ethane gas, and have maximal drift times near $1 \mu \mathrm{s}$. Each drift tube, or cell, has one anode wire varying in length between 1.8 and 6 meters, depending on the detector. Despite the large cell sizes and the long drift times (with respect to the $396 \mathrm{~ns}$ beam crossing), the muon detectors enjoy a relatively low occupancy due to their placement at large radii and behind many $\lambda_{I}$ of absorbers. For all chambers except the CMX, charge division is used to obtain the Z-position of the muons. The signal is read out at both ends of the anode, and the charge asymmetry is related to the location of the avalanche with respect to the center of the wire: $Z / L=\left(Q_{2}-Q_{1}\right) /\left(Q_{1}+Q_{2}\right)$ where $2 \times L$ is the length of the wire, $Q_{1}$ and $Q_{2}$ are the charges collected at the ends, and $Z$ ranges from $-L$ to $L$. The drift cells in the CMX have small angles with respect to each other, and thus provide a full spacepoint measurement without the need for charge division.

The Central Muon Detector (CMU), located outside the CHA, consists of 144 modules of 16 rectangular drift cells. Each module has four radial layers of four cells running in the Z-direction. Alternating layers have a small offset in $\phi$ to provide different sets of drift times for two tracks that pass through symmetrically opposite locations with respect to the anode of a given cell. The modules themselves are arranged in a cylindrical barrels centered about the beam axis, with two $226 \mathrm{~cm}$ modules making up one $452 \mathrm{~cm}$ "stave" of the barrel.

The Central Muon Upgrade (CMP) covers the same region $|\eta|<0.6$ as the $\mathrm{CMU}$, but lies behind another $60 \mathrm{~cm}$ of steel absorber. It also has four layers of cells with a half-cell staggering in $\phi$. The lower occupancy of the CMP allows for $640 \mathrm{~cm}$ long drift tubes that span the entire length of the detector. The CMP itself is rectangular in shape, as shown in Figure 2.9. On the outer surface of the $\mathrm{CMP}^{14}$, there are tiles of scintillators (called the CSP) that are used for triggering

\footnotetext{
${ }^{14}$ With respect to the interaction point.
} 
on muons that pass through the CMU and CMP. These scintillators are necessary for triggering since the drift times are much longer than the beam crossing intervals. It should be noted the CDF trigger does, in fact use information from the drift tubes, but the scintillator hits act as a trigger for the full muon information to be read out ${ }^{15}$.

The Central Muon Extension, (CMX) provides coverage in the pseudorapidity region $0.6<|\eta|<1.0$, except for a $30^{\circ}$ gap in coverage at the top of the detector due to spacial constraints. The detector consists of two truncated cones about the beam line on either end of the central calorimeters, with the small radius ends pointing away from the interaction region, as shown in Figure 2.9. The cones are made of four logical layers of drift tubes, with two overlapping physical layers per logical layer. The physical layers have a small angle relative to each other such that there is total overlap at the small-radius end, and no overlap at the large-radius end. This arrangement is required to build a cone from rectangular cells. A benefit, however, is that the stereo angle enables 3-D spacial resolution without the need for charge division. The inner and outer sides of the cones are covered with layers of trapezoidal scintillators (called the CSX) that are used for triggering.

In the forward/backward region, the Barrel Muon Detectors (BMU) provide coverage up to $|\eta|<2.0$. The BMU consists of two barrels at the ends of the detector. The barrels are made of cells similar to those in the CMP. At the outer radius of the barrels, there are scintillating tiles similar to the ones on the CMP. Each barrel also has a pinwheel-like scintillator counters at the inner end. The counters, shown in Figure 2.19, are projective with respect to the interaction point. Triggering is only possible in the region $|\eta|<1.5$ due to high occupancy in the forward/backward regions ${ }^{16}$. It should be noted that $|\eta|<1.5$ corresponds to $|\theta|<25^{\circ}$ and covers $\sim 90 \%$ of the solid angle.

\footnotetext{
${ }^{15}$ The trigger system is described in more detail later in this chapter.

${ }^{16}$ The occupancy of the detectors is constant in eta, but as $\frac{\delta \eta}{\delta \theta}$ increases dramatically in the forward/backward region, the physical occupancy becomes too high for triggering.
} 


\begin{tabular}{|ccccc|}
\hline \hline & CMU & CMP/CSP & CMX/CSX & BMU \\
\hline Pseudo-rapidity coverage & $|\eta| \leq 0.6$ & $|\eta| \leq 0.6$ & $0.6 \leq|\eta| \leq 1.0$ & $1.0 \leq|\eta| \leq 1.5$ \\
Drift tube cross-section & $2.68 \times 6.35 \mathrm{~cm}$ & $2.5 \times 15 \mathrm{~cm}$ & $2.5 \times 15 \mathrm{~cm}$ & $2.5 \times 8.4 \mathrm{~cm}$ \\
Drift tube length & $226 \mathrm{~cm}$ & $640 \mathrm{~cm}$ & $180 \mathrm{~cm}$ & $363 \mathrm{~cm}$ \\
Max drift time & $800 \mathrm{~ns}$ & $1.4 \mu \mathrm{s}$ & $1.4 \mu \mathrm{s}$ & $800 \mathrm{~ns}$ \\
Total drift tubes & 2304 & 1076 & 2208 & 1728 \\
Scintillation counter thickness & & $2.5 \mathrm{~cm}$ & $1.5 \mathrm{~cm}$ & $2.5 \mathrm{~cm}$ \\
Scintillation counter width & & $30 \mathrm{~cm}$ & $30-40 \mathrm{~cm}$ & $17 \mathrm{~cm}$ \\
Scintillation counter length & & $320 \mathrm{~cm}$ & $180 \mathrm{~cm}$ & $180 \mathrm{~cm}$ \\
Total counters & & 269 & 324 & 864 \\
$\lambda_{I}$ of absorber before component & 5.5 & 7.8 & 6.2 & $6.2-20$ \\
Minimum detectable muon $p_{T}$ & $1.4 \mathrm{GeV} / \mathrm{c}$ & $2.2 \mathrm{GeV} / \mathrm{c}$ & $1.4 \mathrm{GeV} / \mathrm{c}$ & $1.4-2.0 \mathrm{GeV} / \mathrm{c}$ \\
Multiple scattering resolution & $12 \mathrm{~cm} / p$ & $15 \mathrm{~cm} / p$ & $13 \mathrm{~cm} / p$ & $13-25 \mathrm{~cm} / p$ \\
\hline \hline
\end{tabular}

Table 2.2: The CDFII muon system.

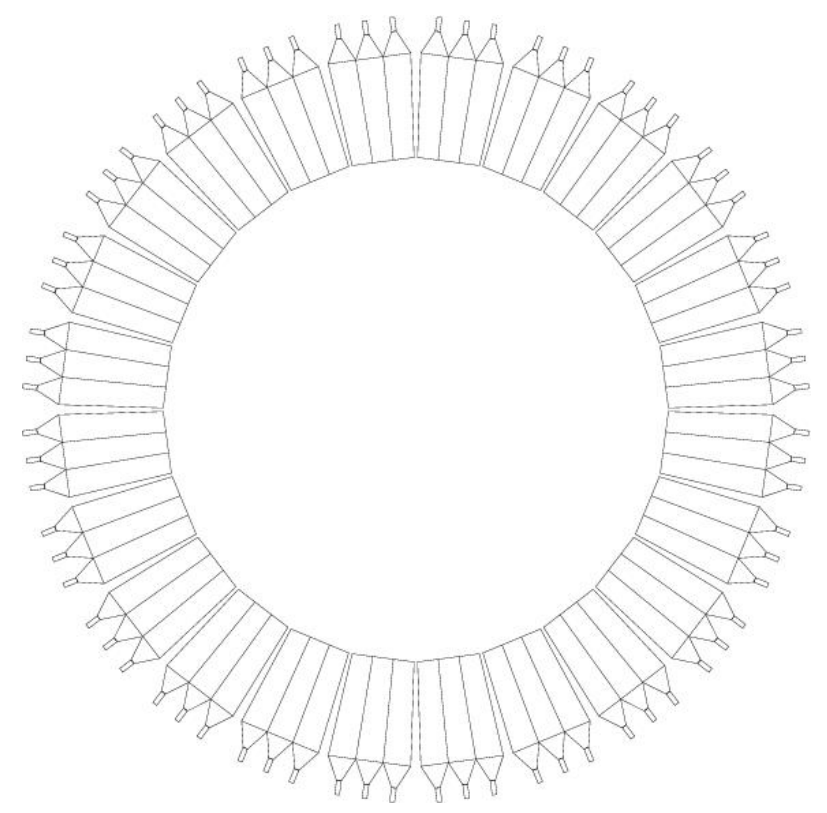

Figure 2.19: A pinwheel-shaped array of scintillators located on the inner edge of the BMU is used for triggering.

\subsubsection{The Luminosity Monitor}

The Cerenkov Luminosity Monitor (CLC) directly counts the number of inelastic $p \bar{p}$ collisions inside the CDF detector. The detector consists of two modules located in the extreme forward/backward regions $(3.7<|\eta|<4.7)$ of the endplug calorimeters. Each module consists of 48 conical Cherenkov counters pointing back to the interac- 
tion region and arragned around the beam-pipe in three layers of 16 . The cones are $180 \mathrm{~cm}$ long except for the innermost layer, where the cones are constrained to 110 $\mathrm{cm}$. At the far ends of the cones, there are conical light collectors, which reflect the light into photo multiplier tubes (PMT's) that have gains of $2 \times 10^{6}$. A layer of soft iron shields the PMT's from the residual magnetic field of the CDF spectrometer. The entire structure is enclosed in a thin aluminium cell, which is filled with isobutane, nominally at atmospheric pressure. The number of photoelectrons produced in the gas is proportional to the number of prompt particles coming from the beam. Secondary particles that arise from interactions of beam particles with material (i.e. the beam pipe) are less energetic, and also traverse the cones at large angles - their short paths through the cones result in much less Cerenkov light. In practice the thresholds at the data acquisition (DAQ) level are higher than the signals caused by secondary particles. The time resolution for the Cerenkov counters is $\sim 100$ ps. This translates to a distance resolution of about $3 \mathrm{~cm}$, which is small compared to the size of the luminous region. It is thus possible to count the number of $p \bar{p}$ interactions per beam crossing by clustering CLC hits in time. Information from the CLC is merged with the event-record offline, and is also relayed back in real time to the main Tevatron control room as a measure of collider performance.

\subsubsection{Triggers and DAQ}

The high luminosities and short beam crossing intervals present in Run-II of the Tevatron constitute a considerable DAQ challenge. The complexity of the detector requires large $(\sim 250 \mathrm{~KB})$ event sizes, and the $396 \mathrm{~ns}$ bunch spacing makes for $10^{14}$ beam crossings per year. For every 150,000 beam crossings, only one event can be written to tape. It is essential to have a high rejection rate, as indicated above, yet still retain all the kinds of events that are of interest to the various physics-goals of the collaborators. To this end, CDF has implemented a 3-stage trigger system with increasing levels of sophistication and with significant buffering of events implemented within the detector. The logical flow of information from 
various subdetectors during the triggering process is shown in Figure 2.20.

At Level 1, custom designed hardware can incorporate information from the calorimeters, muon scintillators, and COT. To save time, two to four calorimeter towers are ganged into trigger towers that have a granularity $\Delta \eta \times \Delta \phi$ of $\sim 0.2 \times$ $15^{\circ}$. The tracks in the COT are found by first finding high- $p_{T}$ track segments in each of the four axial superlayers of the COT and tagging them with a mean $\phi$-position. The segments are then linked together in a configuration consistent with a high- $p_{T}$ track emanating from the interaction point. This algorithm has an efficiency of $\sim 96 \%$, and has $p_{T}$ and $\phi_{0}$ resolutions that are adequate for $r$ $\phi$ extrapolation of tracks to calorimeter clusters or to the scintillator tiles of the muon triggers, thereby reducing trigger-level misidentification rates. There are 56 distinct L1 triggers currently implemented. They may involve various combinations of leptons, tracks, and energy depositions in the calorimeter. It is also possible to trigger on the transverse energy sum, or the missing transverse energy $\left(\mathbb{F}_{T}\right)$ in the event, which at this level is the opposite of the vector sum of the transverse energies in the trigger towers: $\mathbb{F}_{T}=\sum_{\text {towers }}{\overrightarrow{E_{T}}}^{17}$. The Level 1 hardware requires $\sim 4 \mu \mathrm{s}$ for full analysis. Each detector element has a 42-cell pipeline for storing events awaiting the Level 1 decision, which happens every beam crossing ${ }^{18}$. The output rate of Level 1 Accept signals (L1A), which is the input rate to Level 2, is about $35 \mathrm{KHz}$.

At Level 2 (L2), a combination of hardware and software analyzes the data in greater detail. The decision takes about $20 \mu s$, and there are four buffers available for events that await the final L2 decision. Although full L2 reconstruction takes 20 $\mu s$, the buffers enable the L2 decision-making process to be split into two stages of roughly equal time-span. The first stage reconstructs the same objects as in L1, but in greater detail. A clustering algorithm is available for the calorimeter, enabling jet reconstruction. The CSM information is available for better identification of

\footnotetext{
${ }^{17}$ The strict definition of $\vec{E}_{T}$ is $\vec{E}_{T}=-\sum_{i} E_{T i} \hat{n}_{i}, i=$ calorimeter tower number with $|\eta|<3.6$ where $\hat{n}_{i}$ is a unit vector perpendicular to the beam axis and pointing from the interaction point to the $\mathrm{i}^{\text {th }}$ calorimeter tower. We also define $\mathbb{E}_{T}=\left|\vec{E}_{T}\right|$. The $\vec{E}_{T}$ is also corrected for muons as they do not deposit all their energy in the calorimeters.

${ }^{18}$ Several events are being analyzed at any given time.
} 
electrons and photons. Information from the silicon detector is used to determine the tracks' impact parameters with similar efficiency and resolution as in offline analysis, enabling the CDF to trigger on hadronic decays of $\mathrm{B}$ mesons. The first stage culminates with the loading of the newly reconstructed objects into the L2 processors' memories. The second stage checks the data to determine which, if any, of the 131 currently implemented combinations of objects required by the various L2 triggers are satisfied. As the various trigger decisions are being made at this stage, the next event is being uploaded and reconstructed. The Level 2 accept rate can be as high as $600 \mathrm{~Hz}$, with total DAQ deadtime well below $10 \%$.

Events passing L2 enjoy full reconstruction in the Level 3 processor farms. Tracks, muon hits, and energy clusters are converted into high level objects such as taus, muons, vertices, etc. There are currently 185 separate combinations, or paths, of L1/L2/L3 triggers that are implemented at Level 3. Events passing L3 are written to tape at about $100 \mathrm{~Hz}$. About $5 \%$ of the events are sent to the CDF control room in real time, where they are analyzed by special software that continually monitors the response of the detector and DAQ system by comparing occupancies of all the detector channels, trigger rates, DAQ deadtime, and the relative frequency and characteristics of well-known physics processes, such as leptonic decays of $Z$ and $W$ bosons, and $\mathrm{J} / \psi$ mesons.

As mentioned above, the trigger system must meet the needs of the hundreds of collaborators who are interested in a wide range of physics. Some processes are very rare, while others are commonplace but still worth studying. Each trigger has an associated "cross section", which is simply the constant of proportionality between the trigger's accept rate and the luminosity. One way to reduce the overall cross section is to apply an acceptable "prescale" to a trigger. This means that the trigger keeps only a fraction of the events that pass its criteria. As an example, there is a "JET20" trigger used to pick up jets with $E_{T}>20 \mathrm{GeV}$ for jet $\rightarrow$ lepton misidentification studies. It has a combined L1 and L2 prescale of $\sim 1000$, so, of every 1000 events passing L1 and L2, only one goes on for consideration at L3. A 


\section{RUN II TRIGGER SYSTEM}

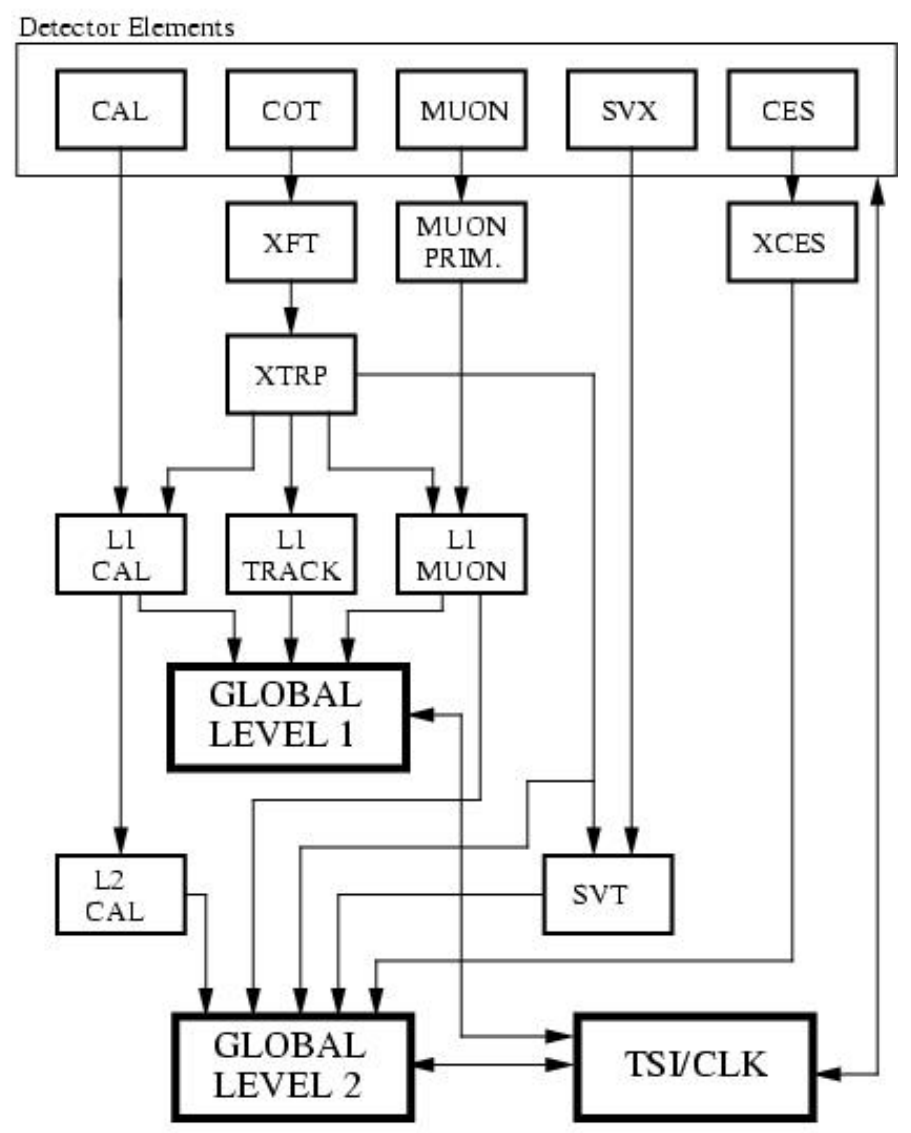

PJW 9/23,96

Figure 2.20: A block diagram of the RunII trigger system.

more extreme example, the "minbias" trigger has no critera whatsoever, but has a prescale of $10^{5}$ at $\mathrm{L} 1(\sim 25 \mathrm{~Hz})$, and is prescaled to $3 \mathrm{~Hz}$ at L2. The luminosity and event rates fall exponentially during a store. CDF uses dynamical prescaling to maximize the physics potential of its triggers. The prescales are initially stringent as the high event-rate threatens substantial $(\sim 8 \%)$ DAQ deadtime, but they are gradually loosened as the luminosity decreases. In this way, the rare and interesting processes that are not prescaled are collected with high efficiency throughout the run, while other more common processes do not suffer from continually high prescales. 


\subsection{Software Tools}

The advent of Monte Carlo (MC) event generators and detector simulations have greatly facilitated analysis in high energy physics. It is possible to generate myriad possible outcomes of high energy beam collisions and to run each such event through a simulation of the detector. The same reconstruction algorithms and analysis techniques can be used on both real and simulated data, and the results can be compared. Event generators break up a physics event into many stages. For example, the cross section $d \sigma / d \Omega$ is calculated for the "hard subprocess", or the inelastic, perturbative part of the event (i.e. $q \bar{q} \rightarrow H^{++} H^{--}$) by evaluating the matrix element - usually to first order. Many such events can be generated by computer, each one weighted according the available phase space for that particular outcome. All unstable particles in the simulation are decayed according to decay tables (i.e. $H^{++} \rightarrow \tau^{+} \tau^{+}$, with final outcomes $\tau_{e} \tau_{h} 23 \%$ of the time...). Initialstate and final-state radiation (ISR and FSR) are incorporated according to the properties of the beam and final-state particles. These calculations do not depend much on the nature of the given hard subprocess, but more so on the makeup of the beam (i.e. the parton distribution functions that characterize the quarks within the protons.), and the overall momentum transfer of the event. Finally, color confinement is imposed by a "fragmentation" scheme which usually involves color-charge singlets pulling appropriate quark-antiquark pairs out of the vacuum to have only color-neutral final-state hadrons. The PYTHIA generator which was used to simulate data samples for this analysis uses the Lund model [27] to simulate fragmentation. In this model, a string is thought to connect the two initially "free" quarks. The string constant ( in analogy with a spring constant) is about $1 \mathrm{GeV} / \mathrm{fm}$. As the quarks move away from each other, they stretch the string, thereby increasing its energy. As enough stored energy becomes available, new $q \bar{q}$ pairs are formed along the string, and the string breaks at a point within the new $q \bar{q}$ pair. This process keeps occurring until there is not enough energy left to generate a new $q \bar{q}$ pair. The result 
is a series of mesons. This scheme is depicted in Figure 2.21. Since the production of these mesons occurs along the string, and the string is localized (to the tune of 1 $\mathrm{GeV} / \mathrm{fm}$ ), the uncertainty principal dictates that the new mesons may have some momentum relative to the string. Due to this momentum, the resultant hadrons travel with some transverse momenta relative to the original quark. The result is a "jet" of hadrons that travel roughly in the direction of the original quark - most jets are confined to a 3 -D cone of $\sim 40^{\circ}$ about the original quark direction, though usually half the jet's energy is contained within some 15 degrees of the original quark's direction. A hadronic jet is depicted in Figure 2.21.

The GEANT[28]-based detector simulation used in CDF has become increasingly sophisticated and accurate due to feedback from continuous calibrations and validation. For example, the simulated profile of EM showers in the calorimeter, or in fact any other detector response to electrons is compared to those from pure samples of electrons obtained from photon conversions, $W, Z$, or $\Upsilon$ events in data. The amount and location of material in the active tracking volume is tuned by comparing maps of photon conversions in data and simulation. The underlying events (the physics not attributed to the hard subprocess) are studied with the aforementioned "minbias" trigger, and used to tune the settings and compare the outputs of various event generators. CDF uses a multi-run monte carlo scheme which adjusts the number of secondary interactions per event according to the instantaneous luminosities in a given dataset. This results in more reliable simulations of effects that depend on detector occupancy. 


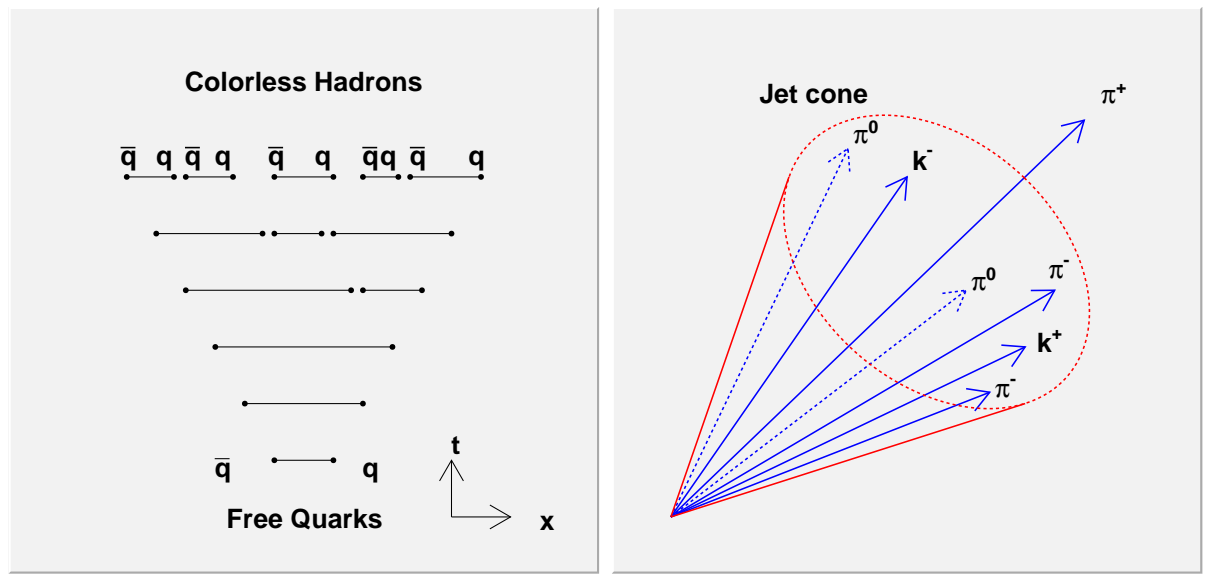

Figure 2.21: (a) A depiction of the Lund model of hadronization. (b) A "jet" of hadrons that results from an initially "bare" quark or gluon. 


\section{Chapter 3}

\section{Search Signature}

\section{1 $H^{ \pm \pm}$Production}

At the Tevatron, the only substantial production mode for the $H^{ \pm \pm}$is assumed to be pair-production through the channel $p \bar{p} \rightarrow H^{++} H^{--1}$. A Feynman diagram of the process is given in Figure 3.1. The leading order (LO) production cross section is given by[30]:

$$
\sigma_{L O}\left(q \bar{q} \rightarrow H^{++} H^{--}\right)=\frac{\pi \alpha^{2} \beta^{3}}{9 Q^{2}}\left[e_{q}^{2} e_{H}^{2}+\frac{\left[e_{q} e_{H} v_{q} v_{H}\left(1-\frac{M_{Z}^{2}}{Q^{2}}\right)+\left(v_{q}^{2}+a_{q}^{2}\right) v_{H}^{2}\right]}{\left(1-\frac{M_{Z}^{2}}{Q^{2}}\right)^{2}+\frac{M_{Z}^{2} \Gamma_{Z}^{2}}{Q^{4}}}\right]
$$

Where $v_{q}=\frac{2 I_{3 q}-4 e_{q} s_{w}^{2}}{2 s_{w} c_{w}}, a_{q}=\frac{2 I_{3 q}}{2 s_{w} c_{w}}, v_{H}=\frac{\left(2 I_{3 H}-4 e_{H} s_{w}^{2}\right)}{2 s_{w} c_{w}}, s_{w}, c_{w}$ are the sines and cosines of the Weinberg angle, $\beta$ is $v / c$ of the $H^{ \pm \pm}, Q^{2}=4 E_{H}^{2}=\sqrt{s}$, e is the eletric charge, and $I_{3 q}$ is the third component of the $H^{ \pm \pm}$'s weak isospin.

This cross section is largely model independent as (3.1) only depends on the electroweak quantum numbers and mass of the $H^{ \pm \pm}$. The cross section for the righthanded $H^{ \pm \pm}$is about half of that for the left-handed $H^{ \pm \pm}$due to the difference in the third component of the weak isospin $\left(I_{3 q}=1\right.$ for $H_{L}^{ \pm \pm}$and $I_{3 q}=0$ for $\left.H_{R}^{ \pm \pm}\right)$. In supersymmetric versions of left-right symmetric models, the production cross

\footnotetext{
${ }^{1}$ It is possible, in certain frameworks, to have a considerable cross section for the process $p \bar{p} \rightarrow$ $H^{++} H^{-}$. This would almost double the production cross section for the $H^{ \pm \pm}$. [29]
} 


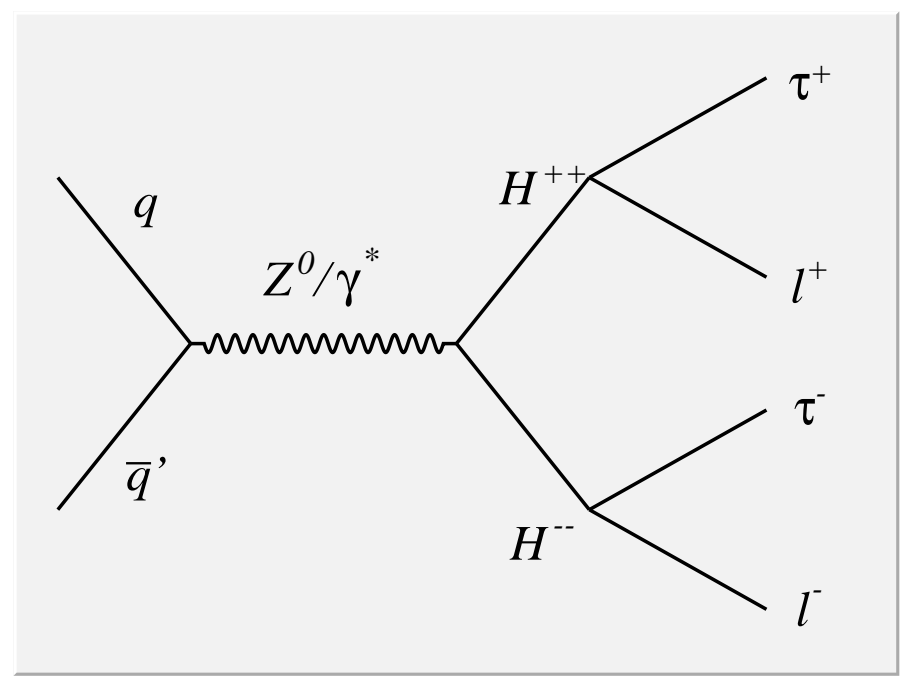

Figure 3.1: The main production mode for $H^{ \pm \pm}$at the Tevatron is pair production through the s-channel.

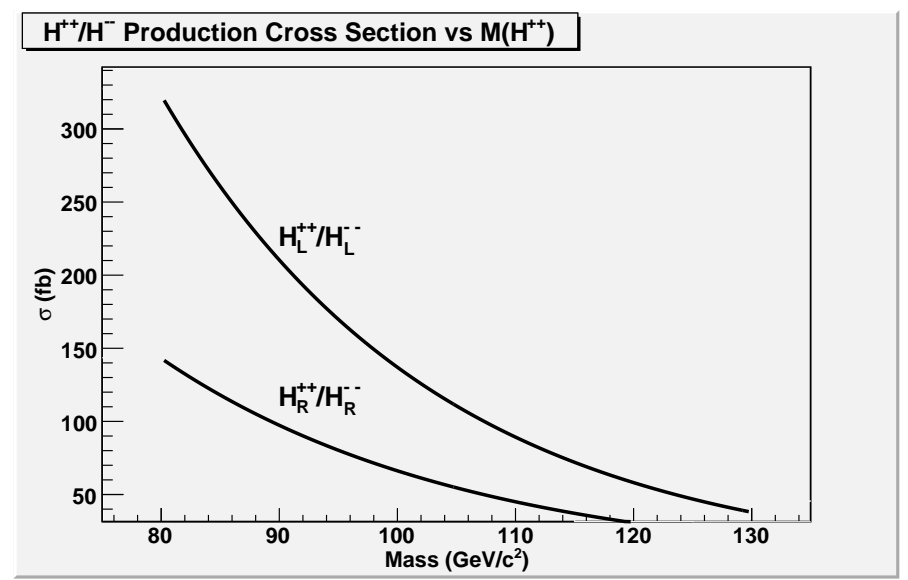

Figure 3.2: The production cross section vs mass for $H^{ \pm \pm}$at the Tevatron.

section for the $\tilde{\Delta}^{ \pm \pm}$- the fermionic superpartners to the $H^{ \pm \pm}$- are significantly enhanced[31]. The ratio of the Next to Leading Order (NLO) to LO cross sections, known as a 'k-factor', is also calculated in [30], and has a value of $\sim 1.3$ for the mass range of interest. The NLO cross sections vs mass for both left and right-handed $H^{ \pm \pm}$are shown in Figure 3.2. 


\section{$3.2 \quad H^{ \pm \pm}$Decay}

The $H^{ \pm \pm}$can couple to charged leptons, $W^{ \pm}$, or $H^{ \pm}$. At masses below 160 $\mathrm{GeV} / c^{2}$ the decay modes that involve $W^{ \pm}$or $H^{ \pm}$are phase-space suppressed[32]. The decay width of the $H^{ \pm \pm}$to any given combination of leptons is given by $\Gamma_{l l^{\prime}}=h_{l l^{\prime}}^{2} m\left(H^{ \pm \pm}\right) /(8 \pi)$, thus the branching ratios to various lepton flavors $(l$ and $l^{\prime}$ ) are determined by the relative strengths of the couplings $h_{l l^{\prime}}$. All combinations of like-sign dileptons $l l^{\prime}$ - even lepton flavor violating combinations are allowed. The branching ratios of $H^{ \pm \pm}$decays into the various leptonic channels are undetermined. In most theoretical frameworks, however, there is reason to expect relatively large branching ratios for decay-modes that involve tau leptons. For example, in the Higgs Triplet Model[29], which is an extension of the SM with a left-handed Higgs triplet, the Lagrangian that describes the interactions between the leptons and the Higgs sector is[33]:

$$
\mathrm{E}_{\text {lepton }}=h_{i j}\left[H^{0} \nu_{i} \nu_{j}+H^{ \pm} \frac{\nu_{i} l_{j}^{\mp}+l_{i}^{\mp} \nu_{j}}{\sqrt{2}}+H^{ \pm \pm} l_{i}^{\mp} l_{j}^{\mp}\right]+h . c .
$$

From (3.2) it is apparent that the same couplings $h_{i j}$ of $H^{ \pm \pm}$to the charged leptons are also proportional to the neutrinos masses, thus, the branching ratio of the $H^{ \pm \pm}$to the leptons will depend on the neutrino mass hierarchy. If one expects the neutrinos to have the same generational mass-hierarchy as the charged leptons and quarks, then the couplings $h_{\tau \tau}, h_{\mu \tau}$ and $h_{\mu \mu}$ will be large [34]. In supersymmetric versions of left-right models[31], the $H^{ \pm \pm}$couple mainly to two like-sign taus ${ }^{2}$ and in much of the SUSYLR parameter space the $\tilde{\Delta}^{ \pm \pm}$decay almost exclusively through the $\tilde{\tau} \tau$ channel because the $\tilde{\tau}$ is the lightest slepton. The $\tilde{\tau}$ will in turn decay through the channel $\tilde{\tau} \rightarrow \tau \tilde{\chi}_{1}^{0}$. In both cases, the signature is 4 taus and missing transverse energy, but with the $\tilde{\Delta}^{ \pm \pm}$the final-state taus are less energetic, and more of the energy is unseen. In left-right symmetric models, the neutrinos obtain mass through the 'seesaw mechanism' [35] and there is no direct mapping

\footnotetext{
${ }^{2}$ The other couplings are experimentally constrained.
} 


\begin{tabular}{|c|c|c|}
\hline \hline Coupling & Maximum Value & Process \\
\hline$h_{e e}$ & 0.3 & Bhabha scattering \\
$h_{\mu \mu}$ & 0.25 & $(g-2)_{\mu}$ \\
$h_{e e} h_{e \mu}$ & $3.2 \times 10^{-7}$ & $\mu \rightarrow 3 e$ \\
$h_{\mu \mu} h_{e \mu}$ & $2 \times 10^{-6}$ & $\mu \rightarrow e \gamma$ \\
$h_{\mu \mu} h_{e \mu}$ & $2 \times 10^{-6}$ & $\mu \rightarrow e \gamma$ \\
$h_{e e} h_{\tau e}$ & $4.3 \times 10^{-3}$ & $\tau \rightarrow e e e$ \\
$h_{\mu \mu} h_{\tau \mu}$ & $3.5 \times 10^{-3}$ & $\tau \rightarrow \mu \mu \mu$ \\
$h_{\mu e} h_{\tau \mu}$ & $5.5 \times 10^{-3}$ & $\tau \rightarrow e \mu \mu$ \\
$h_{\mu e} h_{\tau e}$ & $5.5 \times 10^{-3}$ & $\tau \rightarrow e e \mu$ \\
$h_{e \tau} h_{\tau \mu}$ & $5 \times 10^{-6}$ & $\tau \rightarrow e \gamma$ \\
$h_{\tau \tau}$ & cannot dominate all above couplings & Direct Search \\
\hline
\end{tabular}

Table 3.1: The limits[36] on $H^{ \pm \pm} \rightarrow l l^{\prime}$ couplings from processes that could be mediated by doubly charged particles. The limits depend on $H^{ \pm \pm}$mass and are stated for $m\left(H^{ \pm \pm}\right)=100 \mathrm{GeV} / c^{2}$

of $h_{l l^{\prime}}$ to the neutrino masses. In this case, the couplings are only experimentally restricted. The bounds on $h_{l l^{\prime}}$ are taken from [36] and summarized in Table 3.1. The limits on the couplings $h_{\mu \tau}$ and $h_{e \tau}$ are much less stringent then the other limits. For example, $\frac{h_{\mu e} h_{\tau e}}{h_{\mu e} h_{e e}}=\frac{h_{\tau e}}{h_{e e}} \approx 2 \times 10^{4}$. The ditau coupling $h_{\tau \tau}$ is least constrained by experiment ${ }^{3}$.

\subsection{Search Signature}

The LEP experiments $[37,38,39,40]$ have excluded both $H_{L}^{ \pm \pm}$and $H_{R}^{ \pm \pm}$up to masses of $\sim 100 \mathrm{GeV} / c^{2}$ in the context of the left-right symmetric model[35] and with the assumption of exclusive decays into any given channel. The first results from the Tevatron for $H^{ \pm \pm}$came from the D0 collaboration and excluded $H_{L}^{ \pm \pm}$to $118 \mathrm{GeV} / c^{2}$ assuming exclusive $\mu \mu$ decays of the $H_{L}^{ \pm \pm}$(i.e. $h_{\mu \mu} \neq 0$, all other $h_{l l^{\prime}}=$ 0) [41]. Searches from the CDF collaboration[42] have extended these mass limits to 136,133 , and $115 \mathrm{GeV} / c^{2}$ for $H_{L}^{ \pm \pm}$in the exclusive $e e, \mu \mu$, and $e \mu$ channels respectively. Also, the $H_{R}^{ \pm \pm}$has been excluded to $113 \mathrm{GeV} / c^{2}$ in the $\mu \mu$ channel.

\footnotetext{
${ }^{3}$ Results from LEP [37, 38, 39, 40] have ruled out $e \bar{e} \rightarrow H^{++} H^{--} \rightarrow \tau^{+} \tau^{+} \tau^{-} \tau^{-}$up to $M\left(H^{++}\right) \approx 100 \mathrm{GeV} / c^{2}$. This suggests that at masses near $100 \mathrm{GeV} / c^{2} h_{\tau \tau}$ by itself cannot dominate all the other couplings (e.g. $h_{\tau \tau} \ngtr \sum_{i j}^{i j \neq \tau \tau} h_{i j}$ )
} 
All the aforementioned searches assume that couplings are strong enough (e.g. $h_{l l^{\prime}} \geq$ $\left.10^{-5}\right)$ such that the $H^{ \pm \pm}$decay promptly $(c \tau \leq 10 \mu m)$. For the case that the couplings are very weak and $c \tau \geq 3 m \mathrm{CDF}$ has excluded the $H_{L}^{ \pm \pm}$and $H_{R}^{ \pm \pm}$to 134 $\mathrm{GeV} / c^{2}$ and $109 \mathrm{GeV} / c^{2}$ respectively[22].

As explained above, there is much motivation to search for the exclusive $\tau \tau$ decay mode. However, our simulations showed that the LEP limits could not be approached with our data sample size of $350 \mathrm{pb}^{-1}$. The $\tau \tau$ mode suffers from lower acceptance because the $p_{T}$ spectrum for electrons and muons that come from tau decays is relatively soft. At hadron colliders, the backgrounds for a tau-rich singal are much higher than those for comparable signals involving electrons or muons. Misidentification rates for taus that decay hadronically (hadronic taus, or $\tau_{h}$ ) are two orders of magnitude higher than those for muons or electrons ${ }^{4}$. The combination of lower signal acceptance and higher backgrounds made it impossible for us to reach the LEP limits. As of this writing, CDF has collected over $1.25 \mathrm{fb}^{-1}$. With the larger data sample, it is expected that a dedicated $\tau \tau$ analysis will substantially extend the LEP limits ${ }^{5}$.

The $e \tau$ and $\mu \tau$ decay modes are interesting because the couplings $h_{e \tau}$ and $h_{\mu \tau}$ are much less constrained than the ones that involve only muons or electrons. Furthermore, there is an additional factor of two in the branching ratio of $H^{ \pm \pm} \rightarrow l l^{\prime}: l \neq l^{\prime}$ due to combinatorics. As a side note, although we do not formally set limits in the context of the Higgs Triplet Model [29], it has been shown in [34] that the largest branching ratio would be for the decay $H^{ \pm \pm} \rightarrow \mu^{ \pm} \tau^{ \pm}$if the neutrinos have a normal (increasing with generation) mass hierarchy ${ }^{6}$.

The analysis described in this work extends the LEP mass limits for $H_{L}^{ \pm \pm}$in the exclusive $e \tau$ and $\mu \tau$ channels to 114 and $112 \mathrm{GeV} / c^{2}$ respectively. The lepton-flavor

\footnotetext{
${ }^{4}$ The next chapter explains this in detail.

${ }^{5}$ In the absence of discovery, of course.

${ }^{6}$ Our limits should be directly applicable to $H^{ \pm \pm}$pair production in the HTM. As of now, the standard Monte Carlo generators (i.e. PYTHIA, ISAJET) do not include the $p \bar{p} \rightarrow H^{++} H^{-}$process. It is possible to generate samples with the programs GRACE and GR@PPA, and this should be done in the context of future $H^{ \pm \pm}$searches at the Tevatron. The mass limits in this context should be even stronger than those set for the left-right symmetric model[29].
} 
violating $e \tau(\mu \tau)$ modes have two high $p_{T}$ electrons (muons) and a pair of taus per event. The $p_{T}$ and $|\eta|$ spectra for leptons resulting from decays of $110 \mathrm{GeV} / c^{2}$ $H^{ \pm \pm}$are shown in Figure 3.3. Previous $H^{ \pm \pm}$searches at the Tevatron used a likesign dilepton signature. Standard model processes rarely yield genuine like-sign dileptons - only leptonic decays of ZZ and ZW can yield like-sign leptons ${ }^{7}$. The relatively high jet $\rightarrow \tau_{h}$ misidentification rate increases backgrounds from $W+$ jets and $Z+$ jets processes by roughly two orders of magnitude and precludes a search in the like-sign diletpton channel, as demonstrated in Figure 3.4. In order to suppress jet-based backgrounds, we search for three or more leptons. Although the acceptance for the signal decreases with each additional lepton requirement, the backgrounds decrease dramatically. We split the signal into two separate categories - one has three leptons (we call this category the "3-track" region), and another has four(we call this category the " 4 -track" region $)^{8}$ leptons. Most of the backgrounds in the 'three lepton' region have two genuine leptons (e.g. from a leptonic decay of a $Z$, or leptonic decays of $t \bar{t}$ events) and a misidentified jet. The 'four lepton' region has very low backgrounds, mostly from all leptonic decays of $Z Z$ events. The combined signal acceptance for the three and four lepton channels is about half the average for the $e e, \mu \mu$, and $e \mu$ like-sign dilepton searches. The background levels end up being less than $50 \%$ of the average of the like-sign dilepton searches. This is a great improvement over the situation depicted in Figure 3.4, where our signal to noise ratio was lower than that of the like-sign dilepton analyses by about a factor of 100 .

\footnotetext{
${ }^{7}$ Semileptonic decays of B's in $t \bar{t}$ events, along with leptonic decays of W's can result in like-sign dileptons, however, one of the leptons will be a secondary lepton in a B-jet, and can thus be easily distinguished from prompt leptons (e.g. from electroweak processes.)

${ }^{8}$ Actually, we split up the signal into a three-lepton region, and a region with four or more leptons. It is possible to identify more than four leptons in an $H^{++} H^{--}$event. The fifth lepton will most likely be a hadronic jet (i.e. from ISR gluon radiation) misidentified as a lepton.
} 

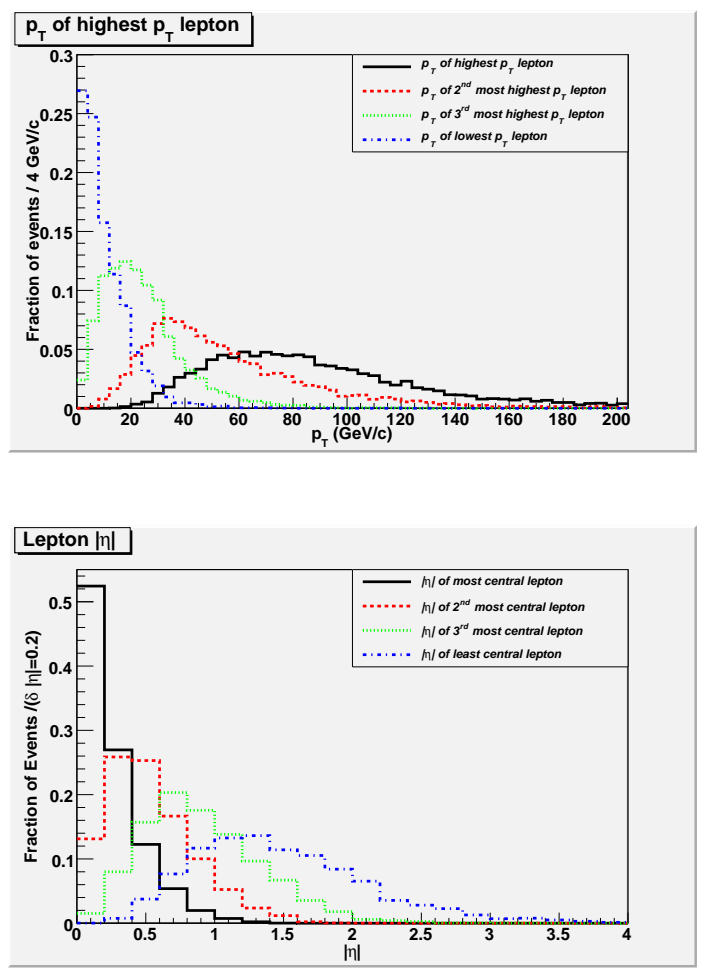

Figure 3.3: (a) The ordered $p_{T}$ distributions for the four leptons that result from the decays of the $\mathrm{H}^{++} \mathrm{H}^{--}$pair. (b) The ordered pseudotapidity distributions for the four leptons. 

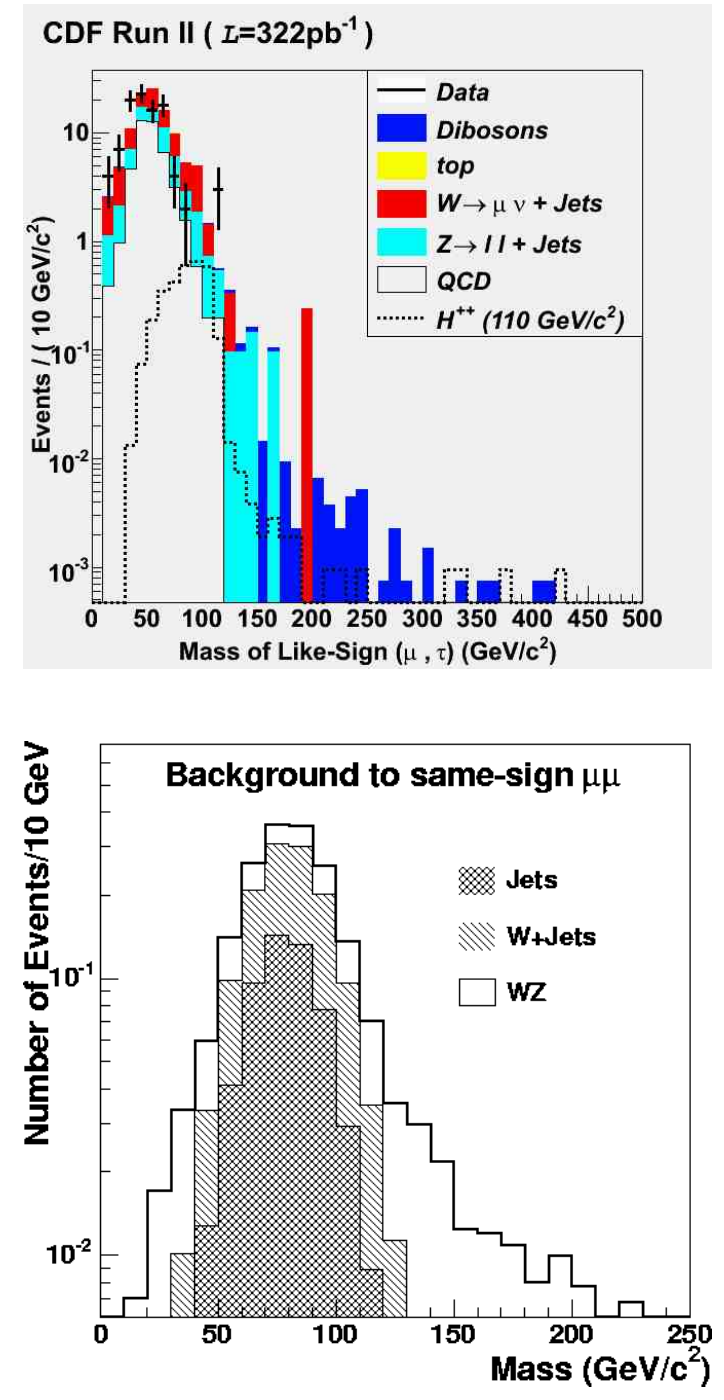

Figure 3.4: (a)The invariant mass of like-sign $\mu+\tau_{h}$ pair for signal, backgrounds, and data. (b) The invariant mass of the SM backgrounds alone for like sign $\mu+\mu$ backgrounds, taken from [42]. 


\section{Chapter 4}

\section{Event Selection}

We begin with a discussion of the reconstruction and identification of leptons and follow with descriptions of kinematical selections involving multiple leptons and the $\mathbb{F}_{T}$, designed to increase the relative content of $H^{ \pm \pm}$in our datasets.

\subsection{Lepton Reconstruction}

Leptons are key final-state particles in many interesting physics processes at hadronic colliders. Various new phenomena would likely be seen in multi-leptonic final states ${ }^{1}$. Leptons are also important because most particles produced at the Tevatron are hadrons produced in jets. It is, as discussed below, possible to distinguish muons and electrons from hadronic jets with such resolution that only one per 10,000 jets is misidentified as an electron or muon. Two primary factors contribute to this rate. There is roughly a factor of 0.01 arising from the fact that electrons and muons interact with the detector in fundamentally different ways than hadrons do. The other factor of 0.01 is due to an isolation requirement - leptons from decays of $W \mathrm{~s}$ or $Z \mathrm{~s}$, for example, have no nearby particles except for the rare cases $(\sim 10 \%)$ when a particle from the underlying event, or perhaps from a jet produced in associaton with the $W$ or $Z$, overlaps with the lepton. Hadronic jets, on the other hand, have

\footnotetext{
${ }^{1}$ Notably SUSY, esp. at high $\tan \beta$, and left-right symmetric models.
} 
a relatively large particle multiplicity. Typically at most one member of a jet will satisfy the lepton ID requirements. The rest of the nearby particles, however, will betray the lepton candidate as a component of a hadronic jet. Isolation requirements can be tuned to reject $\sim 99 \%$ of jets while retaining $\sim 90 \%$ of genuine leptons from electroweak processes. Taus decay before they interact with any component of the detector, and the decay-daughters are pions almost $2 / 3$ of the time. Most finalstate hadrons at CDF are also pions, so only isolation and kinematical selections can be used to distinguish taus from jets. Accordingly, the fake rates for hadronically decaying taus $\left(\tau_{h}\right)$ are roughly two orders of magnitude higher than those for electrons or muons. Many new physics processes, however, such as SUSY, LRSM, and HTM, potentially favor final states involving mixtures of $\tau_{h}$ and one or more of the lighter flavor leptons which may be decay daughters of other tau leptons in the event. Identifying taus, then, is a major part of this work.

\subsubsection{Reconstruction of Electrons}

As mentioned in section 2.4.4, electrons undergo cycles of Bremsstrahlung (brem) with the emitted photon producing $e^{+} e^{-}$pairs, and the resulting $e^{+}$and $e^{-}$also radiating and so on. The radiation length, $X_{0}$, is given by Equation 2.5 and is $6 \mathrm{~mm}$ in lead. The short $X_{0}$ means that electromagnetic showers have narrow profiles (3 or fewer calorimeter towers) and are fully contained in the electromagnetic calorimeters. Electrons are reconstructed both in the CEM $(|\eta|<1)$ and in the PEM $(1.1 \leq|\eta| \leq 2.0)$. In this analysis, we consider only electrons reconstructed in the pseudorapidity range $\left|\eta_{\text {detector }}\right|<1.3$, mainly because, due to the central nature of our signature, the additional acceptance from forward electrons is too small $(\sim$ $1.4 \%)$ to justify the additional backgrounds that come from the consideration of PEM electrons. We describe electron reconstruction in the CEM below. Electron reconstruction in the Plug region is almost identical to that of the CEM, except that, in the region $|\eta|>1.3$ the tracks are mainly reconstructed in the silicon detector rather than in the COT. 
Electron reconstruction[43] begins with the formation of a cluster in the CEM. First, towers are arranged in order of decreasing electromagnetic transverse energy, $E_{T}$, where $E_{T}=E_{\text {tower }} \times \sin \theta_{\text {tower }}$. The clustering algorithm then descends the list of towers, and considers each as the seed tower for a cluster. Three conditions must be met for a tower to be considered as a cluster seed:

- The tower has EM $E_{T}>2 \mathrm{GeV}$.

- The tower must have Had/EM ratio $E_{H a d} / E_{E M}<0.125$ or $E_{E M}>100 \mathrm{GeV}$.

- The tower has not already been included in some other EM cluster.

Once a seed is found, the two towers that are adjacent in $\eta$ to the seed tower are added to the cluster if their energy is above $100 \mathrm{MeV}$. Note that at this stage both the EM and hadronic energies are used to decide whether a tower is added to the cluster - a bias towards low Had/Em ratios would result otherwise.

After clustering, all tracks that extrapolate to within $28 \mathrm{~cm}$ of the seed tower's center in the $r-\phi$ direction and within $38 \mathrm{~cm}$ in the $r-z$ direction, both at the CSM radius of $184 \mathrm{~cm}$, are associated with the electron object. Finally, strip and wire clusters found in the CSM within the towers of the EM cluster are added to the electron object. The CSM, located at $6 X_{0}$ within the CEM, has wires that run along the $z$-direction and cathode strips that run in the azimuthal direction. The distance between adjacent wires is $1.4 \mathrm{~cm}$, and the interstrip distance is $2.0 \mathrm{~cm}$. The default size of CSM clusters corresponding to electrons is 11 strips $\mathrm{x} 11$ wires, and the centroid of the cluster can be found to a precision of less than $1 \mathrm{~cm}$ by weighting the positions of individual wires(strips) within a cluster by the charge collected on that wire(strip).

The collection of CEM towers, CSM clusters, and tracks comprise a reconstructed electron candidate. The energy of the electron is taken from the EM cluster, and the Energy-momentum 4 -vector is $(E \sin \theta \cos \phi, E \sin \theta \sin \phi, E \cos \theta, E)$, where $\theta$ and $\phi$ are taken from the direction of the highest $p_{T}$ track associated with the electron object (called the "seed track"). Corrections are applied to the electron's energy to 
account for variation in response at different locations within a calorimeter tower, tower-to-tower relative gain variations, and the global energy scale of the CEM. These corrections are usually at the $1 \%$ level.

\subsubsection{Reconstruction of Muons}

Muons have the same charge, spin, and weak isospin as electrons, but have a mass of $105.6 \mathrm{MeV}$, or 207 times that of the electron. The rate of energy loss through Bremsstrahlung is inversely proportional to $E / m^{2}$, where $E$ is the particle's energy, and $m$ is its mass, so Bremsstrahlung is a negligible source of energy loss for muons at CDF. Also, because leptons do not carry a color charge, muons do not cause hadronic cascades. As a result, muons are minimally ionizing, and are the only charged particles that are able to penetrate the entire CDF detector ${ }^{2}$.

Muon reconstruction begins with the formation of a muon "stub". A stub is a collection of hits in the muon drift chambers that are consistent with a signal that would be left by a charged particle. The muon detectors have four physical (in the case of the CMX, four logical) layers of long, rectangular drift tubes. Alternating layers are offset by half the width of a drift tube, so that layers 1 and 3 (in order of increasing radius) are aligned together, and layers 0 and 2 are aligned together but offset from layers 1 and 3 . Individual hits in the drift tubes are cataloged by a stubfinding algorithm. The $r-\phi$ coordinate of a hit is related to the physical location of the drift tube in the experiment, while the $r-z$ coordinate is obtained by charge division - with the exception of the CMX, where both $r-\phi$ and $r$ - $Z$ coordinates are related to the drift-tube location. All possible line segments are made using hits in layers 1 and 3 . After the line segments are constructed, layers 0 and 2 are searched for hits that are consistent with the previously formed line segments. If both layers 0 and 2 have hits that are consistent with the line segment made from layers 1 and 3 , then a 4-hit stub candidate is made. If there is only one hit in layers 0 or 2

\footnotetext{
${ }^{2}$ Occasionally, a hadron will make it past the $\sim 7 \lambda_{I}$ of absorbers in CDF. Such hadrons are called "punch-through" hadrons. They are sufficiently rare that the hadron $\rightarrow$ muon fake rate is $10^{-4}$.
} 
consistent with the line segment, a 3-hit stub candidate is made - 2-hit stubs are not considered. The procedure is repeated, this time using layers 0 and 2 as the seeds for the line-segments. After the initial stub-candidates are formed, a drift model[44] is used to more accurately relate the drift time of a hit to the trajectory of the charged particle that passed through the tube. Two least-squares linear fits are made with hits in the stub - one for the $r$ - $\phi$ direction, and the other for the $r$ - $z$ direction. The stub finding in the CMX[45] is similar to the procedure described above, however there are variations because of the different geometry of the detector.

After stubs are found, tracks are extrapolated to the radii of the muon chambers. Any track that comes within $|\Delta x|<30 \mathrm{~cm}$ of a CMU or CMP stub (usually, by $\Delta x$ we mean $\Delta(r-\phi)$ or, at a constant radius, $r \Delta \phi)$, and has a $z$-coordinate consistent with the $z$-span of the detector is associated with the muon object. For the CMX, tracks with $|\Delta x|<50 \mathrm{~cm}$ and with $z$-coordinates that are within the CMX are associated with the muon object. In practice, only one track will typically extrapolate to match the stubs of genuine muons. The highest $p_{T}$ track that extrapolates to the stubs is called the "seed track". Finally, the seed track is extrapolated to the inner and outer radii of the EM and Hadronic calorimeters. The towers that the track passes through are associated with the muon candidate. It should be noted that "stubless" muons are reconstructed in this way: high $p_{T}$ tracks that do not extrapolate to any stubs are still extrapolated through the calorimeters. Any track with $p_{T}>10 \mathrm{GeV} / c$ is a stubless muon candidate. Usually, at the analysis level, tracks with $p_{T}>20 \mathrm{GeV} / c$ and associated calorimeter cluster $E_{T}$ s below $6 \mathrm{GeV}$ are considered as stubless muons. Tracks that pass through fiducial regions of muon detectors but fail to leave stubs are removed from consideration as stubless muons ${ }^{3}$.

The charge of the muon is taken from the curvature of the highest $p_{T}$ track associated with the muon (in most cases, there is usually just one track). The energy-momentum is also taken from the track, with the assumption that the mass

\footnotetext{
${ }^{3}$ This last convention is more popular among analyses that must avoid fake-rates higher than $10^{-4}$. In this work we put no such stipulations on isolated tracks, and rely more heavily on eventlevel kinematical selections to reduce backgrounds.
} 


\begin{tabular}{|cc|}
\hline \hline Decay Mode & Branching Percentage \\
\hline 1-Prong Modes : & $\mathbf{4 5 . 6 4 \%}$ \\
$\pi^{-} \pi^{0} \nu_{\tau}$ & $25.41 \%$ \\
$\pi^{-} \nu_{\tau}$ & $11.06 \%$ \\
$\pi^{-} 2 \pi^{0} \nu_{\tau}$ & $9.17 \%$ \\
$\pi^{-} 3 \pi^{0} \nu_{\tau}$ & $1.08 \%$ \\
\hline $\mathbf{3 - P r o n g}$ Modes $\mathbf{: ~} \mathbf{1 4 . 4 3 \%}$ \\
$\pi^{-} \pi^{+} \pi^{-} \nu_{\tau}$ & $9.11 \%$ \\
$\pi^{-} \pi^{+} \pi^{-} \pi^{0} \nu_{\tau}$ & $4.24 \%$ \\
\hline$e^{-} \overline{\nu_{e}} \nu_{\tau}$ & $17.84 \%$ \\
$\mu^{-} \overline{\nu_{\mu}} \nu_{\tau}$ & $17.36 \%$ \\
\hline
\end{tabular}

Table 4.1: The main decay modes of the tau lepton.

of the particle is $105.6 \mathrm{MeV} / \mathrm{c}^{2}$.

\subsubsection{Reconstruction of Tau Leptons}

\section{Decays of Tau Leptons}

The Tau lepton shares the same spin, electric charge, and weak isospin as the electrons and muons, but has a mass of $1777 \mathrm{MeV}$, and a mean lifetime of $\sim 3 \times 10^{-12}$ s. The tau decays predominantly to one charged and $\geq 0$ neutral pions and a tau neutrino. The next largest decay mode is to three charged and $\geq 0$ pions and a tau neutrino. Together, these two decay modes modes. called "1-Prong" and "3-Prong" respectively, make up over $60 \%$ of the tau's decay width. Decays to electrons $\left(\tau_{e}\right)$ and muons $\left(\tau_{\mu}\right)$ (and neutrinos) make up most of the remaining decay width. The main decay-modes of the tau are shown in Table 4.1. If there are two taus in an event the probabilities for various combinations of decay modes are shown in Table 4.2. It is clear that one must include the hadronic decay modes of taus $\left(\tau_{h}\right)$ in order to maximize acceptance for signatures involving taus. One must first understand the $\tau_{h}$ signature and the relevant backgrounds.

\section{Tau Reconstruction}

Taus that decay hadronically typically consist of one or three charged pions and zero to three neutral pions. The main backgrounds to hadronic taus are "jets" that 


\begin{tabular}{|cc|}
\hline \hline Decay Mode & Branching Percentage \\
\hline$\tau_{h} \tau_{h}$ & $42.1 \%$ \\
$\tau_{e} \tau_{h}$ & $23.1 \%$ \\
$\tau_{\mu} \tau_{h}$ & $22.5 \%$ \\
$\tau_{\mu} \tau_{e}$ & $6.2 \%$ \\
$\tau_{e} \tau_{e}$ & $3.2 \%$ \\
$\tau_{\mu} \tau_{\mu}$ & $3.0 \%$ \\
\hline
\end{tabular}

Table 4.2: The decay probabilities for a pair of tau leptons.

result from the hadronization of free quarks or gluons as discussed before. The difference in particle multiplicity and kinematics between generic hadronic jets and taus is depicted in Figure 4.1. One major difference between jets and taus is that the particles in a tau jet are much more localized than the particles in a hadronic jet. Studies at CDF have shown that hadronic jets typically deposit only $50 \%$ of their energies in a cone of $\Delta R \equiv \sqrt{\Delta \eta^{2}+\Delta \phi^{2}} \leq .175[46]^{4}$. In contrast, hadronic taus deposit nearly $100 \%$ of their energies in cones of the same or even smaller sizes. Figure 4.2 shows that $\sim 95 \%$ of hadronic taus have calorimeter clusters that consist of 6 or less towers, while $\sim 95 \%$ of hadronic jets have calorimeter clusters of 7 or more towers. Also, taus typically have one or three tracks with $p_{T}>1 \mathrm{GeV} / c$, and a net charge of \pm 1 . This is quite distinct from the track multiplicities of hadronic jets, as shown in Figure 4.3. With these distinctions in mind, we describe first the reconstruction and then the selection of tau candidates.

Tau reconstruction begins with the clustering of calorimeter towers that have significant energy depositions in the event. A "seed" tower with $E_{T}>6 \mathrm{GeV}$ is required for tau reconstruction. Clustering starts with the highest $E_{T}$ tower in the event as the seed. If any adjacent tower has $E_{T}>1.0 \mathrm{GeV}$, it is added to the cluster, and all towers adjacent to it are also considered for clustering. The tau clustering algorithm does not consider clusters that have more than 6 towers with

\footnotetext{
${ }^{4}$ Although $\Delta R \equiv \sqrt{\Delta \eta^{2}+\Delta \phi^{2}}$ is not a 3-D angle, in the most central region, where the $\eta-\theta$ correspondence is nearly 1 -to- $1, \Delta R=0.175$ corresponds to a $3-\mathrm{D}$ angle of $10^{\circ}$.
} 


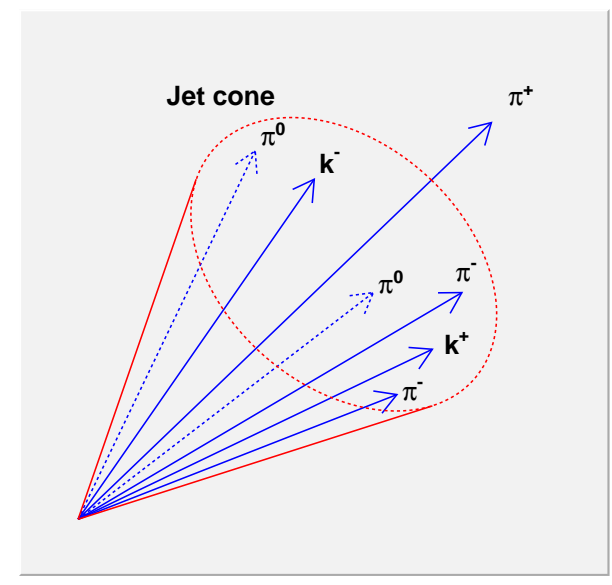

(a)

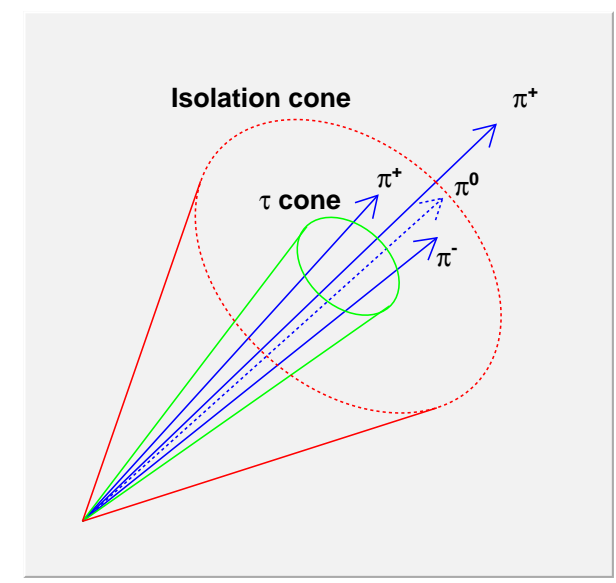

(b)

Figure 4.1: (a) A typical hadronic jet with a large particle multiplicity distributed over a relatively large solid angle. (b) A "hadronic" tau decay, with three charged pions and a neutral pion localized to a relatively small solid angle.

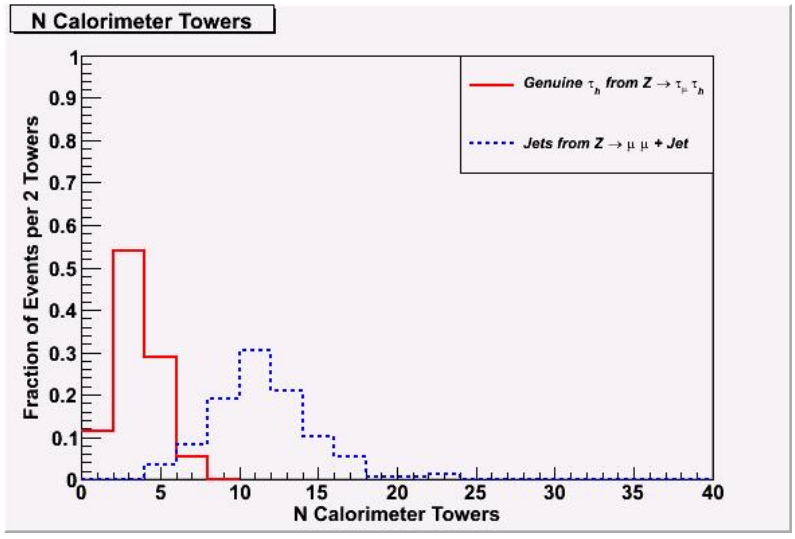

Figure 4.2: The number of towers per calorimeter cluster for genuine hadronic taus from a sample enriched with $Z \rightarrow \tau_{\mu} \tau_{h}$ (solid red) and jets taken from $Z \rightarrow \mu \mu+$ jet events in data (dashed blue).

\section{$E_{T}>1 \mathrm{GeV}$.}

After a cluster has been found, all tracks with $p_{T}>5 \mathrm{GeV} / c$ in the event that have reconstructed segments in at least two axial and two stereo COT superlayers ${ }^{5}$ are queried for a "seed track". The seed track is the highest $p_{T}$ track in the event

\footnotetext{
${ }^{5}$ A COT superlayer consists of 12 sense wires and 13 field shaping wires. A track segment within a superlayer must have at least five associated hits on sense wires.
} 


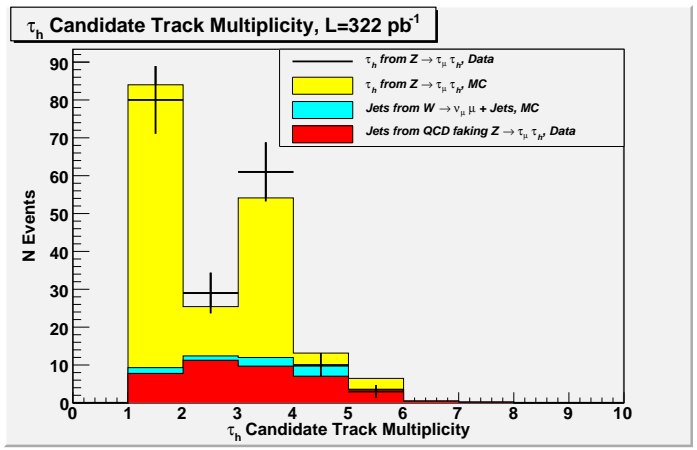

Figure 4.3: The track multiplicity in a $30^{\circ}$ cone about the seed track for tau candidates from $Z \rightarrow \tau_{\mu} \tau_{h}$ in data. Backgrounds are shown from $\mathrm{W}+\mathrm{Jets}$ (from MC) and QCD (from data).

that is extrapolated to pass through the towers in the calorimeter cluster. All other tracks with $p_{T}>1 \mathrm{GeV} / c$ that originate from the same interaction vertex as the seed track, and that have segments in at least two axial and two stereo superlayers, and, are within a 3-D angle $\alpha_{t r k}$ of the seed track, are associated with the tau candidate. For Run I analyses, the angle $\alpha_{t r k}$ was determined to be $10^{\circ}$. In Run II, tau analyses are using a "shrinking cone" to define the localization of the tau. The rationale is that, as taus become more boosted, their decay daughters should become more collinear in the lab frame. This is demonstrated in Figure 4.4, which shows the largest angle between the seed track and any other track associated with a tau object vs the tau energy for samples of simulated taus as well as for a relatively ( $\sim 90 \%)$ pure sample of taus from data and a sample of jets from data. The cone-size adjusts according to the following formula:

$$
\alpha_{\text {trk }}=\min \left[0.175, \max \left(5 \mathrm{GeV} / E^{\text {calo }}, 0.05\right)\right]
$$

where $E^{\text {calo }}$ is the energy in $\mathrm{GeV}$ of the calorimeter cluster associated with the tau candidate, and all other units are in radians. $E^{\text {calo }}$ should be proportional to the tau's boost $\left(E=\gamma m c^{2}\right)$. The minimal cone size corresponds to $2.9^{\circ}$, and applies to taus with energies above $100 \mathrm{GeV}$. 


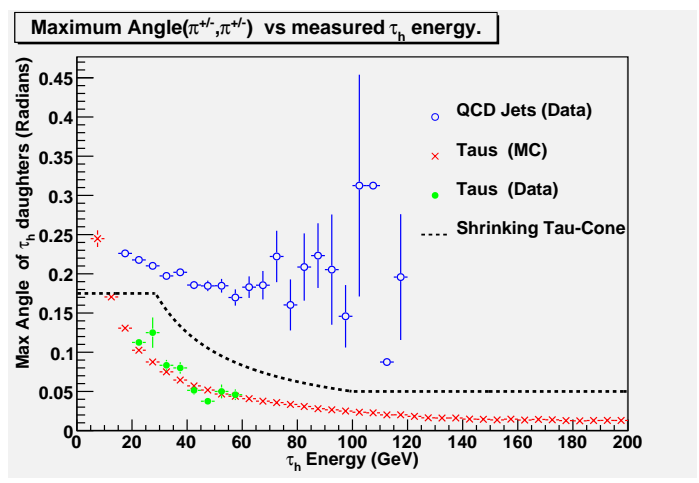

Figure 4.4: The average maximum angle between the highest $p_{T}$ track and another track associated with the reconstructed tau object, versus the reconstructed energy of the tau object. The green solid points represent taus in a $Z \rightarrow \tau_{h} \tau_{l}$ sample in data, the blue open circles represent jets from $\mathrm{QCD}$, while the red $\times \mathrm{s}$ are for a Monte Carlo sample of taus.

Finally, neutral pion candidates are reconstructed in the CSM for all towers that are in the tau cluster. The reconstruction of $\pi^{0} \mathrm{~s}$ starts with the construction of 5strip and 5-wire clusters in the $\mathrm{CSM}^{6}$. The wire and strip clusters within a CEM tower are matched to each other and form 2D clusters in the CSM. If there are more than two candidates for a match, the strip-wire pair whose energies are most compatible are clustered together. Clusters that are within $3 \mathrm{~cm}$ of the trajectories of tracks with $p_{T}>1 \mathrm{GeV} / c$ are rejected as $\pi^{0}$ candidates because neutral pions should not have any associated tracks. If there is just one cluster in a calorimeter tower, then the entire electromagnetic energy of the tower is assigned to the $\pi^{0}$ candidate, except for a small amount that is subtracted to account for the energy deposition of all tracks that traverse the tower:

$$
E^{\pi^{0}}=E_{\text {tower }}^{E M}-\sum_{\text {trk }}\left(0.3 \mathrm{GeV}+0.21 c \times p_{\text {trk }}\right)
$$

where $p_{t r k}$ is the magnitude of the track momentum in units of $\mathrm{GeV} / c$. The sec-

\footnotetext{
${ }^{6}$ The following discussion also applies to the reconstruction of single photons in the CSM. The size of a 5 -strip of 5 -wire cluster in the CSM is roughly $10 \mathrm{~cm}$. The two photons from $\pi^{0}$-decay usually overlap and form one cluster. It is possible to distinguish single photons from neutral pions at low energies, but it is of no interest to this analysis. In any case, the overwhelming majority of localized CSM clusters within tau candidates are in fact due to neutral pions.
} 
ond term in the right-hand side of (4.2) is obtained from studying the calorimeter response to isolated charged pions. If there are more than one $\pi^{0} \mathrm{~s}$ reconstructed within a tower, the tower's EM energy minus the amount calculated by (4.2) is assigned to the $\pi^{0} \mathrm{~S}$ in proportion to their relative cluster energies in the CSM. In defining the $\pi^{0} \mathrm{~s}^{\prime}$ momenta, it is assumed that the $\pi^{0}$ originated from the same primary vertex as the rest of the particles associated with the tau-candidate. All reconstructed neutral pions that are within $10^{\circ}$ of the seed track are associated with the tau object. We do not use the shrinking cone for $\pi^{0} \mathrm{~s}$. The reason is not that the spacial resolution for neutral pions reconstructed in the CSM is poor - clustering enables position resolution of $\sim 1 \mathrm{~cm}$, which, at the CSM radius of $1.4 \mathrm{~m}$, translates to an angular resolution of $\sim 0.01$ radians. The larger cone size is due to a feature in the $\pi^{0}$ reconstruction algorithm. The $\pi^{0}$ clustering algorithm is hard-coded to find 5-strip clusters. If a physical cluster is larger than 10 strips, then two adjacent logical clusters are found with their centers roughly 5 -strips $(\sim 7 \mathrm{~cm})$ apart. At the $\mathrm{CSM}$ radius, this $7 \mathrm{~cm}$ distance corresponds to an angle of 0.05 radians. A software filter was written to correct this feature by merging these "split-off" clusters. At the time of this analysis the filter had not been sufficiently validated and thus tau analyses solved the problem by opting for a 0.175 fixed cone. Figure 4.5 depicts $\pi^{0}$ reconstruction in the CSM.

The matched calorimeter cluster, tracks, and $\pi^{0} \mathrm{~s}$ comprise a reconstructed tau candidate, or a ${C d f T a u^{7}}^{7}$ Several variables can be formed with the information described above and used to select and characterize tau candidates. These variables are described in the next section, but first we discuss the energy measurement for hadronic taus.

It is possible to construct a 4 -vector by adding the 4 -vectors of the tracks associated with the tau candidate. It is assumed that the tracks correspond to charged

\footnotetext{
${ }^{7} \mathrm{CdfT} T a u$ is the name of the $\mathrm{C}++$ object that corresponds to a tau candidate.
} 


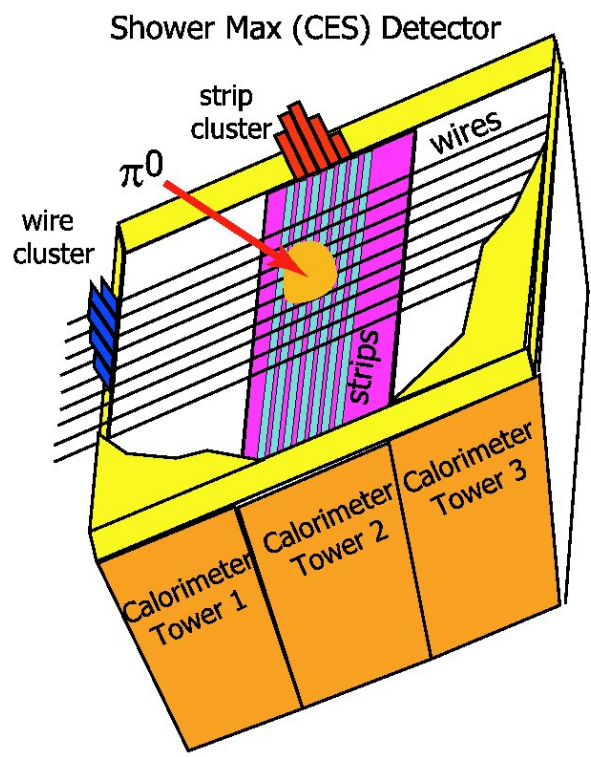

Figure 4.5: A depiction of $\pi^{0}$-reconstruction in the CSM.

pions, thus a 4-vector for an individual track has the form

$$
\left(p_{x}^{t r k}, p_{y}^{t r k}, p_{z}^{t r k}, \sqrt{p_{x}^{2}+p_{y}^{2}+p_{z}^{2}+0.140^{2}}\right),
$$

where the units are in $\mathrm{GeV}$. Likewise, the $\pi^{0} \mathrm{~s}$ reconstructed in the CSM are assigned 4 -vectors of the form $(p \sin \theta \cos \phi, p \sin \theta \sin \phi, p \cos \theta, E)$ where $\phi$ is taken from the cluster position, $\theta$ is taken from the cluster position and from the vertex $z$-position of the tau seed track, $E$ is the energy assigned to the $\pi^{0}$-candidate, $p=\sqrt{E^{2}-0.135^{2}}$ and all units are in $\mathrm{GeV}$.

The sum of the 4-vectors of all the charged and neutral pions is a good measurement of a tau candidate's "visible" energy-momentum, and is in fact the default measurement of the tau's 4-momentum:

$$
\vec{p}^{\tau} \equiv \sum_{\Delta \Theta<\alpha_{t r k}} \vec{p}^{ \pm}+\sum_{\Delta \Theta<\alpha_{\pi^{0}}} \vec{p}^{0}
$$

It is impossible to reconstruct the full momenta of actual tau leptons because the neutrinos associated with tau-decays do not interact with the detector. For this rea- 
son, (4.4) is called the "visible" energy-momentum of the tau, or in short $p_{t r k s+\pi^{0} s} \equiv$ $p_{V i s}$. This hybrid quantity uses information from the COT and CEM/CSM, and yields a better measurement of the tau's visible energy than that obtained by pure calorimetry. The improvement comes from relying on tracking rather than calorimetry for the measurement of the charged pions' energies, because hadronic calorimeter's energy resolution, particularly for small clusters, is not nearly as good as those of the tracking chamber or the EM calorimeter. The only drawback to using $p_{\text {trks }+\pi^{0} s}$ is that sometimes a $\pi^{0}$ is not reconstructed, either due to instrumental effects or because a charged pion is within $3 \mathrm{~cm}$ of the $\pi^{0}$-cluster. The latter effect becomes more significant in the high- $p_{T}$ regime, where the pions are highly localized in the lab frame. An energy-correction algorithm, described in the appendix, has been created to compensate for this effect. The energy resolution for 1-prong and 3-prong taus is plotted in Figure 4.6 for $E_{C a l}, E_{t r k s+\pi^{0} s}$, and corrected $E_{t r k s+\pi^{0} s}$ measurement as returned by the energy-correction algorithm. 


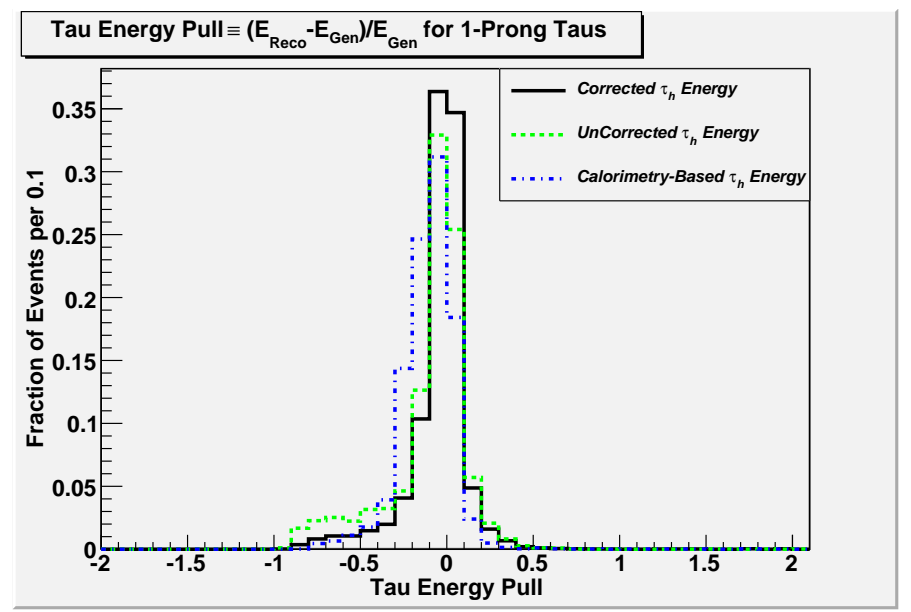

(a)

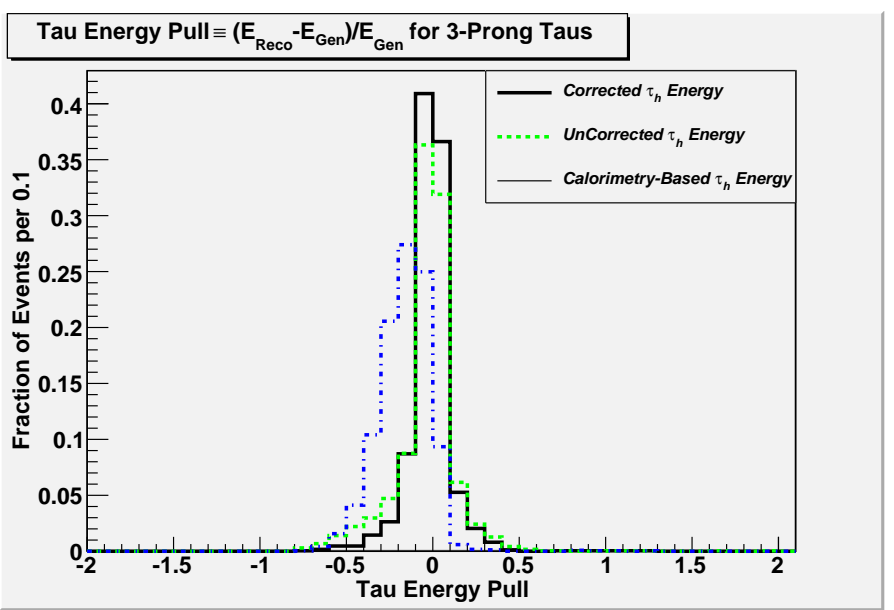

(b)

Figure 4.6: (a) The energy resolution for simulated 1-prong hadronic taus resulting from $H^{ \pm \pm}$decays as determined by calorimetry(dot-dashed) and by tracks and $\pi^{0} \mathrm{~s}$ with(solid) and without(dashed) the energy correction algorithm. Note how the correction algorithm corrects the low-energy tail. (b) The same plot for 3-prong taus.

\subsection{Lepton Identification}

The goal of lepton reconstruction is to provide a collection of all objects that are

likely to correspond to leptons. One must apply various selections to reject spuri- 
ous lepton candidates while retaining well-measured, genuine leptons. Any particles that are not the lepton of interest, but pass all identification selections are called "fake" leptons. Every search or measurement has its own particular set of background processes that mimic the signal by supplying either genuine or fake leptons, photons, and jets. The lepton identification selections can be chosen to reach an optimal combination of signal acceptance and background rejection. If the major backgrounds do not rely heavily on misidentification of leptons, or if they can be later suppressed with kinematical cuts, then it makes sense to choose lepton identification selections that maximize the acceptance for signal. On the other hand, if the backgrounds are kinematically similar to the signal, and rely heavily on lepton misidentification, then more stringent lepton ID selections must be applied.

\section{Lepton Isolation}

We briefly discuss the concept of isolation here because it is common to the identification of leptons of all flavors. Many interesting processes involve the production of one or more heavy particles, followed by the decay of each heavy particle into one or two charged leptons, and perhaps some neutral particles. Typical examples from the standard model are $Z, W$, and $Z W$ and $Z Z$ production followed by leptonic decays. Examples from models beyond the standard model include production of $\tilde{\chi}_{1}^{ \pm} \tilde{\chi}_{1}^{0}$ followed by leptonic decays, and also the signatures investigated in this work. Typically, these events have a low particle multiplicity because most of the available energy goes into the production of one or two heavy particles that decay into just one or two leptons that are usually well-separated from each other. We expect final-state leptons from such processes to be isolated, that is, there should not be particles in the event with trajectories similar to those of the leptons in the event. Isolation can be defined and measured in different subdetectors. The typical implementation involves an isolation cone of half-angle $\sqrt{(\Delta \theta)^{2}+(\Delta \phi)^{2}}$ about the direction of the given lepton, within which the multiplicity and energies of particles

other than the given particle must be small. Most quark or gluon jets are results 
of fragmentation, and involve a large number of particles that travel in the same direction as the original quark or gluon. Figure 4.7 shows the distribution for muon candidates from both electroweak and QCD events.

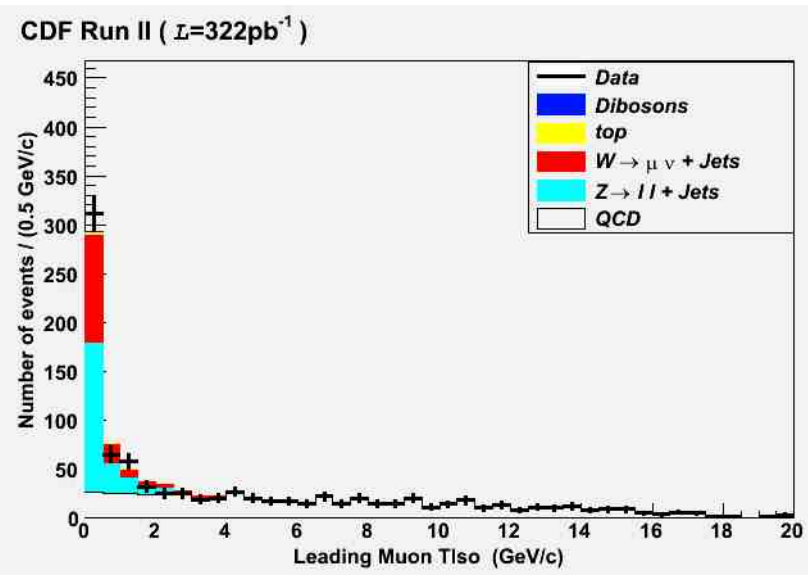

Figure 4.7: The track isolation distribution for leading muons in a $322 \mathrm{pb}^{-1}$ data sample. The TIso is defined as the sum $p_{T}$ of all tracks within cone of half-angle $\left(\sqrt{(\Delta \theta)^{2}+(\Delta \phi)^{2}}\right)$ of 0.4 radians about the muon's track. The sample consists of events that remain after selection of one muon and one tau. All selection cuts are applied except for muon TIso. The electroweak processes are mostly in the range TIso $<2 \mathrm{GeV} / c$, while most of the QCD-based events (plotted in gray) have TIso $>2 \mathrm{GeV} / c$.

\subsubsection{Electron Identification}

Backgrounds to electrons can be $\pi^{ \pm}$that undergo charge exchange (e.g., $\pi^{+}+N \rightarrow$ $\pi^{0}+P$ ) in the EM calorimeter, or overlapping $\pi^{ \pm} \pi^{0}$ combinations with the $\pi^{ \pm}$ providing the track and the $\pi^{0}$ providing the EM cluster. Also, electrons that result from $B$-decays or from photon conversions may be backgrounds to "prompt" electrons that result directly from decays of signal particles (e.g. $\left.H^{++} \rightarrow e^{+} e^{+}\right)$. In principle, the same stipulations used to reject fakes emanating from jets also work on semileptonic B-decays and conversions of photons that result from $\pi^{0}$ decays within jets. The $H^{++} H^{--} \rightarrow e^{+} \tau^{+} e^{-} \tau^{-}$signature has at least two high $E_{T}$ electrons. We require that at least one electron to pass the following selections, which are summarized in Table 4.3. 


\section{- Central Detector Region}

The EM cluster must be fully contained in the CEM. All towers in the EM cluster must belong to the CEM. This requirement is equivalent to imposing $\left|\eta_{D e t}\right|<1.0$, where $\eta_{\text {Det }}$ is the $\eta$ of the centroid of the EM shower associated with the electron.

\section{- Transverse Energy $\mathbf{E}_{T}$}

The transverse energy of the electron candidate, given by $E_{T}=E_{C E M} \times$ $\sin \theta_{t r k}$, with $E_{C E M}$ as the energy of the EM cluster and $\theta_{t r k}$ taken from the electron's track, must be greater than $20 \mathrm{GeV}$. This requirement is efficient for our signal, which has two prompt, high $p_{T}$ electrons from $H^{ \pm \pm}$-decays, but helps to suppress fakes from jets.

\section{- Transverse Momentum $\mathbf{p}_{T}$}

The seed track's $p_{T}$ must be greater than $10 \mathrm{GeV} / c$.

\section{- CSM Fiduciality}

The electron candidate must pass through the instrumented volume of the CSM. The seed track is extrapolated to the CSM radius of $184 \mathrm{~cm}$. The zcoordinate of the point of extrapolation should be in the range $9<\left|z_{R_{C S M}}^{t r k}\right|<$ $230 \mathrm{~cm}$, and the local $x$-coordinate $(r \Delta \phi(\operatorname{trk}, C S M))$ should be $\left|x_{r_{C S M}}^{t r k}\right|<21.5$ $\mathrm{cm}$.

\section{- COT Fiduciality}

The electron candidate must pass through all four axial superlayers of the COT. The electron's track is extrapolated to the COT exit-radius of $137 \mathrm{~cm}$. The $z$-coordinate of this point must be $\left|z_{R=137 \mathrm{~cm}}^{t r k}\right|<150 \mathrm{~cm}$.

\section{- Track Quality Requirements}

The seed track must be well reconstructed in the COT - it must have hits in at least 5 of 12 sense wires in at least 3 axial superlayers and at least 2 stereo superlayers. 


\section{- Track Impact Parameter}

The seed track's impact parameter $d_{0}$ is the shortest distance between the fitted track helix and the beamline ${ }^{8}$. The "raw" $d_{0}$ is defined as $\sqrt{x^{2}+y^{2}}$ in CDF coordinates, and assumes a perfectly aligned beam. The location of the beam, however, is not actually at $x=0=y$. The $x$ and $y$ coordinates of the interaction vertices for each run are kept in a database, and the "raw" $d_{0}$ is compensated for the $x$-y offset of the beam. The corrected $d_{0}{ }^{9}$ must be less than $1 \mathrm{~cm}$. This not only rejects poorly reconstructed tracks, but also rejects particles (i.e. kaons) that may have decayed in mid-flight.

\section{- Vertex Position}

The distribution of primary vertices about the nominal interaction point $(z=0)$ has a gaussian form with $\sigma=26 \mathrm{~cm}$. The area $|z|<60 \mathrm{~cm}$ is called the "luminous" region, and about $98 \%$ of collisions take place there. We require that the $z_{0}$ of the electron candidate - the $z$-coordinate measured at the same 3-D spacepoint as $d_{0}$ - be within $60 \mathrm{~cm}$ of $z=0$.

\section{- Hadronic-to-EM Energy Ratio}

It is highly unlikely for an electron to penetrate past the EM calorimeter. On the other hand, the EM calorimeter only comprises about 1 nuclear interaction length, compared to 4.5 for the hadronic calorimeter. Hadrons are likely to deposit more energy in the hadronic calorimeter than in the EM calorimeter. The ratio of a particle's energy deposition in the Hadronic calorimeter to its EM calorimeter deposition is called the Hadronic to EM ratio (Had/Em). For electrons, we require:

$$
H a d / E m \leq 0.05+.00045\left(\mathrm{GeV}^{-1}\right) \times E m,
$$

\footnotetext{
${ }^{8}$ Technically, if $d_{0}$ is to be one of the 5 parameters that describe the helical trajectory of a charged particle in a magnetic field, then it must also carry a factor of \pm 1 , depending on whether the track's helix includes or excludes the $z$-axis. We are only interested in the magnitude of the impact parameter, so $d_{0}$ is to be understood as $\left|d_{0}\right|$.

${ }^{9}$ Henceforth $d_{0}$ shall stand for "corrected $d_{0}$ unless otherwise noted.
} 
where Had and Em are the cluster's EM and Hadronic energies measured in $\mathrm{GeV}$. The second term in the right hand side of (4.5) allows for a small amount of leakage into the Hadronic calorimeter for very energetic electrons and stabilizes the cut's efficiency and efficacy with respect to the electron's energy.

\section{- Lshr (Lateral Sharing)}

The lateral shower profile of electrons and photons in the CEM has been documented during test beam studies. The lateral shower sharing variable, lshr [47], compares the observed sharing of energy between towers in the CEM with the amount of sharing that is expected from test beam data. The variable $l s h r$ is defined as:

$$
l s h r=0.14 \frac{\sum_{i}\left(M_{i}-P_{i}\right)}{\sqrt{\left(0.14 \sqrt{E_{E M}}\right)^{2}+\sum_{i}\left(\Delta P_{i}\right)^{2}}}
$$

where the sums are over towers that have same $\phi$ index and are adjacent in $\eta$ to the seed tower. $M_{i}$ is the measured energy in adjacent tower $i$, and $P_{i}$ is the predicted energy in that tower. $E_{E M}$ is the total electromagnetic energy in the cluster, and $\Delta P_{i}$ is an estimate of the uncertainty of $P_{i}$.

Due to the small value of $X_{0}$ compared to the dimensions of the calorimeter towers, there is very little sharing unless an electron traverses near an $\eta$ boundary of a tower, or actually traverses two towers. For electrons and photons, lshr has a small value. On the other hand, most backgrounds to electrons are combinations of charged and neutral pions, with the charged pion supplying a track and the neutral pion(s) supplying a cluster(s) in the EM calorimeter. Such combinations will likely have wider shower profiles because there are at least two particles involved, and also because one of them is likely to have a hadronic shower. We require $l s h r<0.2$ for electrons.

- $\operatorname{CSM} \Delta x$ 
The track and CSM cluster should overlap within the spacial resolution of track extrapolation and CSM cluster reconstruction. As usual, $\Delta x($ trk, cluster $)$ here means $r \Delta \phi($ track, cluster $)$. This requirement is efficient for electrons, but less so for combinations of pions that mimic an electron in the detector, because in these backgrounds the track and CSM cluster are actually coming from different particles. We require $-3 \mathrm{~cm}<Q \times \Delta x<1.5 \mathrm{~cm}$, with $Q$ being the sign of the electron candidate's charge. The asymmetry in the cut-values and the factor of $Q$ account for the added track curvature that may result from Bremsstrahlung.

\section{- $\operatorname{CSM} \Delta z$}

This is track/CSM cluster matching in the $r-z$ direction. The seed track is extrapolated to $r_{C S M}=184 \mathrm{~cm}$, and $\Delta z($ track, cluster $)$ is formed from the $z$-positions of the CSM cluster and extrapolated track. We require $|\Delta z|<3$ $\mathrm{cm}$.

\section{- Energy to Momentum Ratio}

In the massless approximation that holds for highly boosted particles, the magnitudes of energy and momentum should be nearly equal. We require that $E_{C E M} /\left(c p_{t r k}\right)<4$. The "loose" value for this cut increases signal efficiency for electrons that undergo Bremsstrahlung, since the emitted photon and secondary electron will both contribute to $E_{C E M}$, while $c p_{t r k}$ will be reduced. To further increase acceptance, the cut is not applied to those electrons with $p_{T}>50 \mathrm{GeV} / c$.

\section{- Track Isolation (TIso)}

The scalar sum of the $p_{T} \mathrm{~s}$ of all tracks within a cone of 0.4 radians $\left(23^{\circ}\right)$ about the electron candidate's seed track must be less than $2 \mathrm{GeV} / c$.

\section{- Photon Conversion Removal}

We do not explicitly remove conversions as part of electron selection because 
conversion-based backgrounds are negligible to our signal. We do, however, discuss the conversion-tagging algorithm, because conversion-tagging is used to estimate the $\gamma+$ jets background to our signal. It is possible to reconstruct and "tag" electron-positron pairs that come from photon conversions in the tracking volume. To be tagged, the pair are required to be consistent with the decay of a virtually massless particle. The conversion tag has two requirements. Firstly, the conversion-candidates tracks are required to have very similar $\theta$ coordinates: $|\Delta \cot \theta|<0.03$. Secondly, the two track helices are projected onto the $x-y$ plane, and the minimal distance between the two arcs at the point where their tangents are parallel (Figure 4.8), called $S_{x y}$, is required to be less than $0.1 \mathrm{~cm}$.

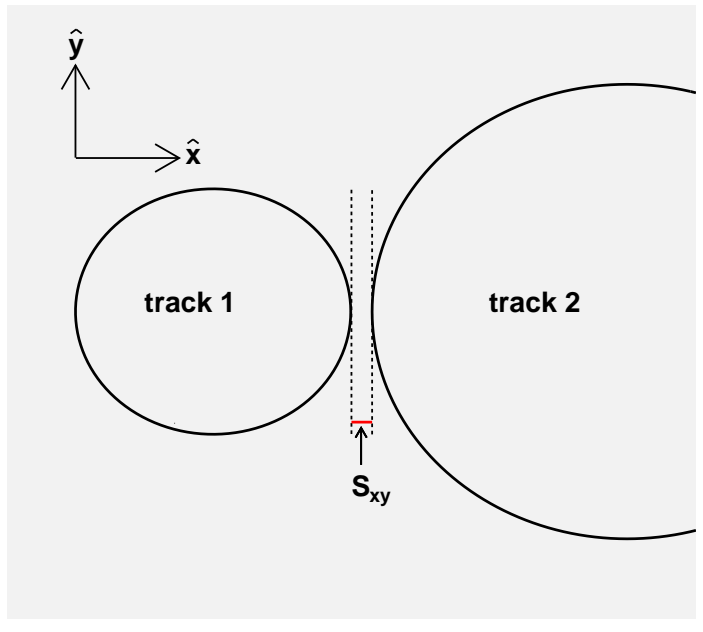

Figure 4.8: A depiction of $S_{x y}$ : the two circles represent the projection of the two track helices onto the $x-y$ plane.

\subsubsection{Muon Identification}

Charged pions that are not fully absorbed in the calorimeter, called "punch through" pions, can become backgrounds to muons if they leave stubs in the muon drift chambers. Most other sources of backgrounds to muons are genuine but misbegotten muons. For example, semileptonic decays of $B$-jets can also result in real muons, but 


\begin{tabular}{|c|}
\hline \hline Electron Cuts \\
$\left|\eta_{\text {Det }}\right|<1.0$ \\
$E_{T} \geq 20 \mathrm{GeV}$ \\
$p_{T} \geq 10 \mathrm{GeV} / c$ \\
Track $z_{0} \leq 60 \mathrm{~cm}$ \\
$\left|z_{r=137 \mathrm{~cm}}^{\text {trk }}\right|<150 \mathrm{~cm}$ \\
$9 \mathrm{~cm}<\left|z_{C E S}\right|<230 \mathrm{~cm}$ \\
$\left|x_{C E S}\right|<21 \mathrm{~cm}$ \\
\hline Had $/ \mathrm{Em}<0.055+.00045 * E$ \\
$\mid \Delta z($ trk, $C E S) \mid<3 \mathrm{~cm}$ \\
$-3<Q \times \mid \Delta x($ trk, CES $) \mid<1.5 \mathrm{~cm}$ \\
Lshr $\leq 0.2$ \\
Track Isolation $(0.4-\mathrm{cone}) \leq 2.0 \mathrm{GeV}$ \\
$\mathrm{E} / \mathrm{P} \leq 4$ if $p_{T}<50 \mathrm{GeV} / \mathrm{c}$ \\
Track $\mathrm{d}_{0} \leq 1.0 \mathrm{~cm}$ \\
Track Quality: $3 / 2 / 5(\mathrm{NAxSeg} / \mathrm{NStSeg} / \mathrm{NHits})$ \\
\hline
\end{tabular}

Table 4.3: Electron ID cuts: The first seven "acceptance" requirements are mainly geometrical and kinematical in nature, while the final eight "ID" cuts check that the particle is consistent with an electron.

those muons are still regarded as backgrounds because they are the decay daughters of $B$ mesons. The $H^{++} H^{--} \rightarrow \mu^{+} \tau^{+} \mu^{-} \tau^{-}$signature has at least two high $p_{T}$ muons. We require that at least one muon to pass the following selections, which are also summarized in Table 4.4.

\section{- Muon Detector Fiduciality}

The muon candidate's track must be extrapolated to pass through the instrumented parts of either the CMX, or both the CMP and CMU.

\section{- COT Fiduciality}

The muon candidate must pass through all four axial superlayers of the COT.

The seed track is extrapolated to the COT exit-radius of $137 \mathrm{~cm}$. The Zcoordinate of this point must be $\left|Z_{R=137 \mathrm{~cm}}^{\text {trk }}\right|<150 \mathrm{~cm}$.

\section{- Stubs}

The muon candidate must either have matching stubs both in the CMU and the CMP (such muons are called "CMUP" muons), or in the CMX. The com- 
bined coverage of these detectors, shown in Figure 4.9 is $|\eta|<1.0$, with some small gaps in the $\eta-\phi$ regions.

\section{- Transverse Momentum $\mathbf{p}_{T}$}

The transverse momentum of the muon's track must be greater than $20 \mathrm{GeV} / c$. This requirement is efficient for our signal, which has two prompt, high $p_{T}$ muons from $H^{ \pm \pm}$-decays, but helps to suppress fakes from jets.

\section{- Track Quality Requirements}

The muon's seed track must be well reconstructed in the COT. The track must have hits in at least 5 of 12 sense wires in at least 3 axial superlayers and at least 2 stereo superlayers.

\section{- Impact Parameter}

We opt for more stringent $d_{0}$ cuts for muons to suppress potential backgrounds from cosmic rays. The seed track's $d_{0}$ must be less than $2 \mathrm{~mm}$. If the track has at least three axial hits in the silicon detector the impact parameter resolution markedly improves, and we require that $d_{0}<0.2 \mathrm{~mm}$ for such cases ${ }^{10}$.

\section{- Vertex Position}

The muon must come from the luminous region. We require that the seed track's $z_{0}$ be within $60 \mathrm{~cm}$ of $z=0$.

\section{- Hadronic Energy}

Muons should be minimally ionizing particles. We require that the total energy in all hadronic towers traversed by the seed track be consistent with minimally ionizing particles:

$$
E_{H A D}<6.0+\operatorname{Max}\left(0,0.028 c \times\left(p_{t r k}-100\right) \mathrm{GeV}\right.
$$

The second term on the right-hand side of (4.7) compensates for Bremsstrahlung.

\footnotetext{
${ }^{10}$ About $90 \%$ of our dataset was taken with the silicon detectors operational.
} 
For muons, radiative effects account for just $1 \%$ of energy-loss at energies of $\sim 60 \mathrm{GeV}$, but grow to $50 \%$ at $\sim 300 \mathrm{GeV}$.

\section{- EM Energy}

The minimally ionizing requirement also holds for the EM calorimeter:

$$
E_{E M}<2.0+c \operatorname{Max}\left(0,0.0115 c \times\left(p_{t r k}-100\right) \mathrm{GeV}\right.
$$

The second term on the right-hand side of (4.8) compensates for Bremsstrahlung at high $p$.

\section{- Track Isolation (TIso)}

The scalar sum of the $p_{T} \mathrm{~s}$ of all tracks within a cone of 0.4 radians $\left(23^{\circ}\right)$ about the muon's seed track must be less than $2 \mathrm{GeV} / c$.

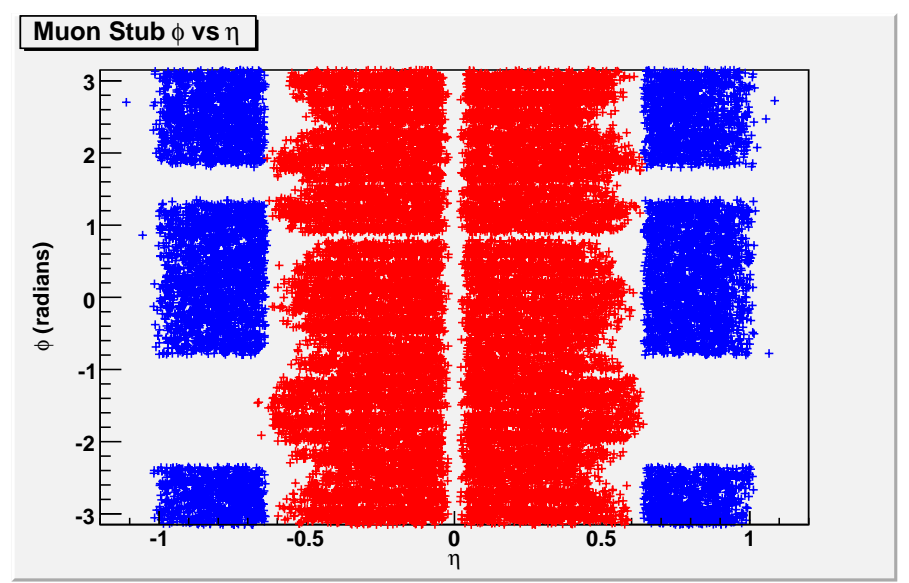

Figure 4.9: The $\phi$ vs $\eta$ coverage of the CMX and CMU/CMP detectors as shown by the presence of muon stubs. The points in the central region correspond to the coincidence of matching CMU and CMP stubs.

\subsubsection{Hadronic Tau Identification}

The identification requirements for hadronic taus are mostly kinematical in nature. Most backgrounds to hadronic taus are jets that result from hadronization of quarks 


\begin{tabular}{|c|}
\hline \hline Muon Cuts \\
\hline \hline Fiducial in CMUP or CMX \\
Stubs in CMUP or CMX \\
$p_{T} \geq 20 \mathrm{GeV}$ \\
$\left|z_{r=137 \mathrm{~cm} \mid}^{\text {trk }}\right| 150 \mathrm{~cm}$ \\
Track $z_{0} \leq 60 \mathrm{~cm}$ \\
\hline HadE $<6.0+\operatorname{Max}(0, .028 *(P-100))$ \\
EmE $<2.0+\operatorname{Max}(0, .0115 *(P-100))$ \\
$\mid \Delta x(t r k$, stub $) \mid<10 \mathrm{~cm}$ \\
Track Isolation $(0.4-\mathrm{cone}) \leq 2.0 \mathrm{GeV}$ \\
Track $d_{0} \leq 0.2 \mathrm{~cm}(\mathrm{No} \mathrm{Si})$ \\
Track $d_{0} \leq 0.02 \mathrm{~cm}(\mathrm{Si})$ \\
Track Quality: $3 / 2 / 5(\mathrm{NAxSeg} / \mathrm{NStSeg} / \mathrm{NHits})$ \\
\hline
\end{tabular}

Table 4.4: Muon ID cuts: The first five "acceptance" requirements are mainly geometrical and kinematical in nature, while the final six "ID" cuts check that the particle is consistent with a prompt muon.

or gluons. The following selections are used to suppress hadronic backgrounds, and also to ensure that a tau candidate is well-reconstructed.

\section{- CSM Fiduciality}

The tau candidate must pass through the instrumented volume of the CSM. The seed track is extrapolated to the CSM radius of $184 \mathrm{~cm}$. The $z$-coordinate of the point of extrapolation should be in the range $9<\left|z_{R_{C S M}}^{t r k}\right|<230 \mathrm{~cm}$, and the local $x$-coordinate $(r \Delta \phi(t r k, C S M))$ should be $\left|x_{r_{C S M}}^{t r k}\right|<21.5 \mathrm{~cm}$.

\section{- COT Fiduciality}

The tau's seed track is extrapolated to the COT exit-radius of $137 \mathrm{~cm}$. The $z$-coordinate of this point must be $\left|z_{r=137 \mathrm{~cm}}^{\text {trk }}\right|<150 \mathrm{~cm}$.

\section{- Track Quality Requirements}

The seed track must have hits on at least 5 of 12 sense wires in at least 3 axial superlayers and at least 2 stereo superlayers. If the tau has more than one track associated with it, the secondary "shoulder" tracks must have at least 5 hits in one axial and one stereo superlayers.

\section{- Track Impact Parameter}


The impact parameter must be less than $2 \mathrm{~mm}$ for the seed track and less than $1 \mathrm{~cm}$ for shoulder tracks.

\section{- Vertex Position}

The tau must come from the luminous region. We require that the seed track have $\left|z_{0}\right|<60 \mathrm{~cm}$.

\section{- Visible Mass}

The visible mass, sometimes called $M^{v i s}$ or $M^{t r k+\pi^{0}}$, is the invariant mass of the 4 -vector defined in equation (4.4). We require $M^{t r k+\pi^{0}}<2.5 \mathrm{GeV} / c^{2}$. This is somewhat higher than the actual $1.777 \mathrm{GeV} / c^{2}$ mass of the tau, however, a more stringent mass limit would be inefficient. The determination of the energy-momenta of the neutral pions (esp. for "split-off" clusters (4.1.3) ) is the limiting factor for tau mass resolution.

\section{- Track Mass}

The track mass, $M^{\text {trks }}$ is the invariant mass of the 4 -vector

$$
p^{\tau-t r k s} \equiv \sum_{\Delta \Theta<\alpha_{t r k}} p^{\pi^{ \pm}}
$$

with the assumption that all tracks correspond to charged pions. We require that $M^{\text {trks }}<1.8 \mathrm{GeV} / c^{2}$.

\section{- Track Multiplicity}

Over $99.9 \%$ of tau decays involve just 1 or 3 charged particles. We require that the tau candidate has 1 or 3 tracks:

$$
\sum_{\Delta \Theta<\alpha_{t r k}} t r k=1, \text { or } 3
$$

with the angle $\alpha_{t r k}$ given by (4.1).

\section{- Charge}

The charge of the tau candidate, taken as the sum of the charges of its tracks, 
must be \pm 1 :

$$
Q_{\tau}=\sum_{\Delta \Theta<\alpha_{t r k}} Q_{t r k}= \pm 1
$$

with the angle $\alpha_{t r k}$ given by (4.1).

\section{- Track Isolation}

The scalar sum of the $p_{T} \mathrm{~s}$ of all tracks that fall inside an isolation cone of half-angle $30^{\circ}$, but outside the tau signal cone, must be less than $2 \mathrm{GeV} / c$ :

$$
I_{t r k}^{\Delta \Theta}=\sum_{\alpha_{t r k}<\Delta \Theta<30^{\circ}} p_{T}^{t r k}<2 \mathrm{GeV} / c
$$

The cone angle $\alpha_{t r k}$ is given by (4.1).

\section{- $\pi^{0}$ Isolation}

The sum $p_{T}$ of all neutral pions that fall between a signal cone of half-angle $10^{\circ}$, and an isolation cone of half-angle $30^{\circ}$, about the seed track, must be less than $0.5 \mathrm{GeV} / c$ :

$$
I_{t r k}^{\Delta \Theta}=\sum_{10^{\circ}<\Delta \Theta<30^{\circ}} p_{T}^{\pi^{0}}<0.5 \mathrm{GeV} / c
$$

\section{- Electron Rejection}

Note that the CdfTau reconstruction algorithm efficiently reconstructs primary electrons, and stores access to its calorimeter, CSM, and tracking information. As a result, a sample of CdfTaus will contain electrons and hadronic taus, as well as narrow (e.g. less than 6 towers in cluster) hadronic jets. Electrons are typically removed from consideration by requiring significant energy deposition in the hadronic calorimeter. To maximize acceptance for our analysis, we do not remove any lepton candidate that is consistent with an isolated electron. We do, however, reclassify tau candidates with Emfr $>0.9$ as "loose" electron candidates, as described in the next section. 


\begin{tabular}{|c|}
\hline \hline Tau Cuts \\
$\left|\eta_{\text {Det }}\right|<1.0$ \\
Track $z_{0} \leq 60 \mathrm{~cm}$ \\
$\left|z_{r=137 \mathrm{~cm}}^{\text {trk }}\right|<150 \mathrm{~cm}$ \\
$9 \mathrm{~cm}<\left|z_{C E S}\right|<230 \mathrm{~cm}$ \\
$\left|x_{C E S}\right|<21 \mathrm{~cm}$ \\
$E_{T}>15 \mathrm{GeV}$ \\
$p_{T}^{\text {seed }}>6 \mathrm{GeV} / \mathrm{c}$ \\
\hline Mass(Tracks) $\leq 1.8 \mathrm{GeV} / \mathrm{c}^{2}$ \\
Mass(Tracks+ $\left.\pi^{0} \mathrm{~s}\right) \leq 2.5 \mathrm{GeV} / \mathrm{c}$ \\
Track Isolation (shrinking-cone) $\leq 2.0 \mathrm{GeV}$ if Emfr $<0.9$ \\
$\pi^{0}$ Isolation $($ fixed-cone $) \leq .5 \mathrm{GeV}$ \\
SeedTrack $\mathrm{d}_{0} \leq 1.0 \mathrm{~cm}$ \\
Track Quality: $3 / 2 / 5(\mathrm{NAxSeg} / \mathrm{NStSeg} / \mathrm{NHits})$ \\
$N_{\text {Tracks }}=1$ or 3 \\
Charge $= \pm 1$ \\
\hline \hline Tau-Cone Definition: \\
Shrinking Cone (for TIso) \\
Signal Cone: $0<\theta<\alpha$ \\
Iso Cone: $\alpha<\theta<0.52$ \\
Max $\left[.005\right.$, Min $\left.\left(0.175,5.0 / E_{C l u s t e r}\right)\right]$ \\
Fixed-Cone (for $\pi^{0}$-iso) \\
$0.175<\theta<0.52$ \\
\hline \hline
\end{tabular}

Table 4.5: Tau idetification selections. The first seven are geometrical and kinematical in nature, while the last eight impose consistency with genuine tau leptons.

\subsubsection{Additional Lepton Types}

To increase signal acceptance, we can relax some of the stringent lepton-ID requirements described in the previous section. Below we describe additional lepton categories that are used in the $e \tau$ and $\mu \tau$ searches.

\section{Loose Electrons or "Electron-Like" CdfTaus}

There are no substantial backgrounds to our search that mimic our signal by supplying spurious electrons. We can increase signal acceptance with little or no increase in background levels by relaxing some of the electron selections. To this end, tau candidates (CdfTaus) that are likely to be electrons are treated as "loose" electrons. They must satisfy selections identical to those in Table 4.5 with the following mod- 
ifications. A tau candidate that has Emfr $>0.9$ is reclassified as a "loose" electron if it passes the above selections with the following three modifications:

- The standard 0.4-cone TIso is used instead of tau-style double-cone track isolation.

- The "track $+\pi$ " mass cut is not used.

- The $\pi^{0}$-isolation region is relaxed from $10^{\circ}<\Theta_{I S O}<30^{\circ}$ to $13^{\circ}<\Theta_{I S O}<30^{\circ}$.

In practice, both the tight hadronic tau, and the loose electron, correspond to a mixture of hadronic taus, electrons, and misidentified hadronic jets. The loose electron, however, due to its high Emfr, has very little hadronic backgrounds. In this work, we use tight hadronic taus or loose electrons interchangably, and refer to them as "tau-like" or "electron-like" CdfTaus respectively.

\section{Loose Tau Candidates}

A Loose Tau Candidate, or LTC, satisfies the same selections as tight taus (Table 4.5) with the following modifications:

- The $E_{T}$ requirement is lowered to $10 \mathrm{GeV}$.

- The $\eta$ requirement is relaxed to $|\eta|<1.3$.

- For LTCs in the pseudorapidity range $1.0<|\eta|<1.3$, the calorimeter energy is used, as $\pi^{0}$-reconstruction is only available in the region $|\eta|<1.0$.

As with the tight CdfTaus, the LTC can be electron-like or tau-like, according to its Emfr (greater than or less than 0.9). The LTC selections are summarized in Table 4.6.

\section{Isolated Track System}

An isolated track system (ITS) is identical to an LTC except it does not use calorimetry at all. An isolated track system results from applying just the track-based parts 


\begin{tabular}{c}
\hline \hline LTC Cuts \\
$\left|\eta_{\text {Det }}\right|<1.3$ \\
Track $z_{0} \leq 60 \mathrm{~cm}$ \\
$E_{T}>10 \mathrm{GeV}$ \\
$p_{T}^{\text {seed }}>6 \mathrm{GeV} / c$ \\
\hline Mass $($ Tracks $) \leq 1.8 \mathrm{GeV} / c^{2}$ \\
Mass(Tracks $+\pi^{0}$ s $) \leq 2.5 \mathrm{GeV} / c$ if Emfr $<0.9$ \\
Track Isolation (shrinking-cone) $\leq 2.0 \mathrm{GeV}$ if Emfr $<0.9$ \\
TIso $0.4 \leq 2.0 \mathrm{GeV}$ if Emfr $>0.9$ \\
$\pi^{0}$ Isolation (fixed-cone) $\leq .5 \mathrm{GeV}$ \\
SeedTrack $d_{0} \leq 1.0 \mathrm{~cm}$ \\
Track Quality: $2 / 2 / 5(\mathrm{NAxSeg} / \mathrm{NStSeg} / \mathrm{NHits})$ \\
$N_{\text {Tracks }}=1$ or 3 \\
Charge $= \pm 1$ \\
\hline \hline Tau-Cone Definition: \\
Shrinking Cone $($ for TIso) \\
Signal Cone: $0<\theta<\alpha$ \\
Iso Cone: $\alpha<\theta<0.52$ \\
Max $\left[.005\right.$, Min $\left.\left(0.175,5.0 / E_{C l u s t e r}\right)\right]$ \\
Fixed-Cone $\left(\right.$ for $\pi^{0}$-iso) \\
$0.175<\theta<0.52$ if Emfr $<0.9$ \\
$0.225<\theta<0.52$ if Emfr $>0.9$ \\
\hline
\end{tabular}

Table 4.6: LTC Selection.

\begin{tabular}{|c|}
\hline \hline ITS Cuts \\
\hline$\left|\eta_{\text {Det }}\right|<1.3$ \\
Track $\mathrm{Z}_{0} \leq 60 \mathrm{~cm}$ \\
$E_{T}>10 \mathrm{GeV}$ \\
$p_{T}^{\text {seed }}>6 \mathrm{GeV} / c$ \\
\hline Mass(Tracks) $\leq 1.8 \mathrm{GeV} / c^{2}$ \\
Track Isolation $(0.175 \mathrm{cone}) \leq 2.0 \mathrm{GeV}$ \\
$\pi^{0}$ Isolation $(0.225$ cone $) \leq .5 \mathrm{GeV}$ \\
SeedTrack $d_{0} \leq 1.0 \mathrm{~cm}$ \\
Track Quality: $2 / 2 / 5(\mathrm{NAxSeg} / \mathrm{NStSeg} / \mathrm{NHits})$ \\
$N_{\text {Tracks }}=1$ or 3 \\
Charge $= \pm 1$ \\
\hline
\end{tabular}

Table 4.7: ITS Cuts

of tau reconstruction and identification. The ITS can correspond to charged leptons of all three flavors. The cuts for the ITS are described in Table 4.7. 


\subsection{Missing Transverse Energy and Related Corrections}

At hadron colliders, the center-of-mass (CM) frames of the inelastic interactions generally have substantial velocities in the Z-direction with respect to the lab frame. This is because incoming quarks, antiquarks, or gluons generally carry unequal fractions of the proton's or antiproton's momenta. The net momentum of the incoming particles in the direction transverse to the beam, however, is practically zero. Any imbalance in the net transverse momentum of the final-state particles is due to imperfect particle detection. For example, neutrinos do not interact with the detector, leading to potentially large apparent energy-momentum imbalances such as those in the leptonic decays of $W$ bosons. Uninstrumented regions of the CDF can can also cause apparent energy-momentum imbalances. For example, CDF lacks calorimeter coverage in the pseudorapidity region $|\eta|>3.6$, so extremely forward/backward particles are not detected. Also, the energies and momenta of particles are measured with finite resolution, leading to imbalances in the net final-state momentum. For example, two back-to-back jets with $E_{T} \mathrm{~s}$ of $100 \mathrm{GeV}$ may be measured at $96 \mathrm{GeV}$ and $103 \mathrm{GeV}$ by the calorimeters, leading to a $7 \mathrm{GeV}$ imbalance. An imbalance in the net transverse momentum is called the "missing transverse energy" $\mathbb{F}_{T} \equiv\left|\vec{E}_{T}\right|$ and is defined by

$$
\vec{E}_{T}=-\sum_{\text {tower }} E_{T i}^{\left|\eta_{i}\right|<3.6} \hat{n}_{i}
$$

where $\hat{n}_{i}$ is a unit vector perpendicular to the beam axis and pointing to the $\mathrm{i}^{\text {th }}$ calorimeter tower. Equation 4.14 needs to be modified for cases where a particle's corrected energy differs from the corresponding energy cluster in the calorimeters. For example, muons deposit a small fraction of their energies in the calorimeters, but their momenta are usually well-measured by the tracking chambers. The $\mathscr{H}_{T}$ as given by (4.14) must be corrected as such:

$$
\begin{aligned}
& E_{x} \rightarrow E_{x}-\left(P_{x}^{\mu}-E_{x}^{\mu}\right), \\
& E_{y} \rightarrow E_{y}-\left(P_{y}^{\mu}-E_{y}^{\mu}\right),
\end{aligned}
$$


where $E_{x}$ and $E_{y}$ are the $x$ and $y$ components of the $\mathbb{F}_{T}, E_{x}^{\mu}$ and $E_{y}^{\mu}$ are the $x$ and $y$ components of the calorimeter energy cluster associated with the muon, and $P_{x}^{\mu}$ and $P_{y}^{\mu}$ are the $x$ and $y$ components of the muon's momentum as measured by the tracking chamber. Energy corrections applied to electrons and to hadronic taus are also propagated into the $\mathscr{F}_{T}$. In this case the correction takes the analogous form,

$$
\begin{aligned}
& E_{x} \rightarrow E_{x}-\left(E_{x}^{C o r r}-E_{x}^{C a l}\right), \\
& E_{y} \rightarrow E_{y}-\left(E_{y}^{C o r r}-E_{y}^{C a l}\right),
\end{aligned}
$$

where $E^{C o r r}$ stands for the corrected energy measurement and $E^{C a l}$ stands for the calorimeter energy deposition associated with the particle. These corrections are validated by comparing corrected and uncorrected $\mathbb{E}_{T}$ distributions for events in data that should not have any corrected $\mathbb{F}_{T}$, such as $Z \rightarrow e e$ and $Z \rightarrow \mu \mu$, and also for events in $\mathrm{MC}$ where the actual $\mathbb{F}_{T}$ due to neutrinos is known. Finally, the MC predictions for corrected $\mathscr{F}_{T}$ are compared to data in various control samples, as explained in the next section. In Figure 4.10 we compare the $x$-component of the corrected and uncorrected $\mathbb{E}_{T}$ with the $x$-component of the neutrino momenta for events generated by the PYTHIA MC program.

\subsection{Datasets}

\subsubsection{Triggers}

Data for this analysis are collected by the "Lepton+Track" triggers. These triggers are designed to be sensitive to multilepton events that may include hadronically decaying taus. As described in section 2.4.7, CDF employs a three-stage triggering system. A specific combination of L1,L2, and L3 triggers is called a trigger path. The ELECTRON8_TRACK5_ISO trigger path was used to collect data for the $e \tau$ search. This trigger requires one track with $p_{T}>5 \mathrm{GeV} / c$ and tau-style isolation in the pseudorapidity region $|\eta|<1$ in addition to an electron with $E_{T}>8 \mathrm{GeV}$, 


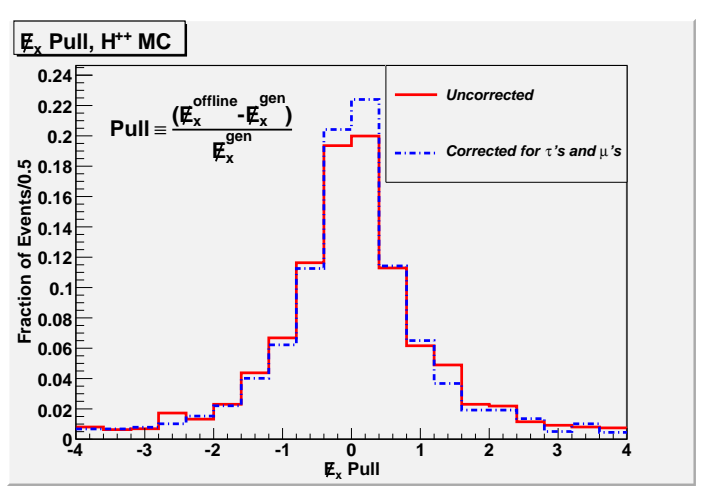

(a)

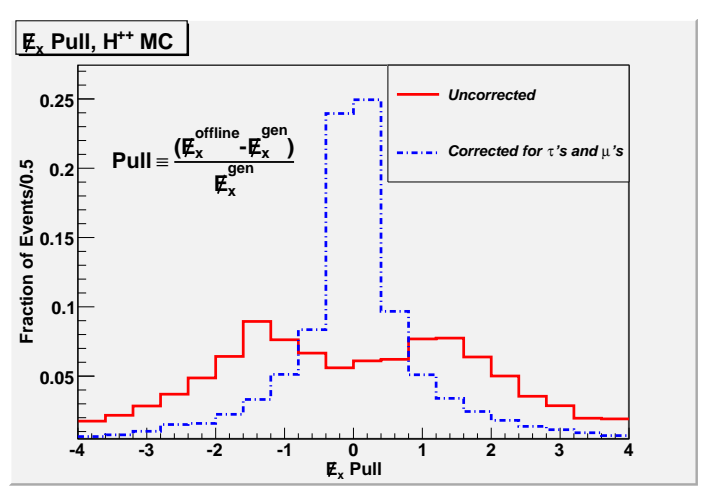

(b)

Figure 4.10: (a) Corrected (dashed blue) and uncorrected (solid red) $E_{x}$ resolution for a $110 \mathrm{GeV} / c^{2}$ signal sample with exclusive $e \tau$ decays. (b) Corrected (dashed blue) and uncorrected (solid red) $E_{x}$ resolution for a $110 \mathrm{GeV} / c^{2}$ signal sample with exclusive $\mu \tau$ decays. The dramatic correction to the $\mathbb{F}_{T}$ is due to the high $p_{T}$ muons.

and $p_{T}^{t r k}>8 \mathrm{GeV} / c$ in the region $|\eta|<1.0$. Detailed requirements of this trigger path are listed in Table 4.8. Two trigger paths are used for the $\mu \tau$ search: the CMUP8_TRACK5_ISO path requires one track with $p_{T}>5 \mathrm{GeV} / c$ and tau-style isolation in the pseudorapidity region $|\eta|<1$ in addition to a muon with $p_{T}^{\text {trk }}>$ $8 \mathrm{GeV} / c$ in the region $|\eta|<0.6$. The CMX8_TRACK5_ISO paths requires one track with $p_{T}>5 \mathrm{GeV} / c$ and tau-style isolation in the pseudorapidity region $|\eta|<1$ in addition to a muon with $p_{T}^{\text {trk }}>8 \mathrm{GeV} / c$ in the region $0.6<|\eta|<1.0$. The 
two muon+track triggers cover a similar $\eta-\phi$ region as the electron+track trigger. Detailed requirements of these trigger paths are listed in Table 4.9.

\section{Trigger Efficiency}

The information available to the trigger logic is stored in the event record. The hardware triggers (and to a lesser extent, the software L3 trigger) make extremely fast decisions, but at the cost of reconstruction efficiency and energy resolution. For example, an electron that has $E_{T}=8.5 \mathrm{GeV}, p_{t}^{\text {trk }}=8 \mathrm{GeV} / c$ offline ${ }^{11}$ might have $E_{T}=8.2 \mathrm{GeV}, p_{t}^{t r k}=8.1 \mathrm{GeV} / c$ at Level 3 , and $E_{T}=7.5 \mathrm{GeV}, p_{t}^{t r k}=8.5 \mathrm{GeV} / c$ at Level 1. Such an electron would fail a trigger requirement $E_{T}>8 \mathrm{GeV}$ at Level 1, even though its offline $E_{T}$ is above threshold. This demonstrates that the efficiency of the trigger relative to the offline is not $100 \%$, and must be measured and properly accounted for to determine the degree of an analysis' acceptance for a given signal.

The trigger efficiency measurement procedure is to find the trigger-level counterparts to offline tracks, calorimter clusters, hits in the muon chambers, etc. and determine what fraction of them satisfy the trigger requirements. The trigger efficiencies are usually parameterized as functions of properties of the offline objects. For example, Figure 4.11 shows the Level 1 electron trigger efficiency vs the offline electron $E_{T}$. The curve very closely resembles a step function $\theta(x-8 \mathrm{GeV})$, which is the ideal trigger design specification. There are three distinct regions to this curve: the "rejection region", where the efficiency is essentially $0 \%$, the "plateu region", where the efficiency is very close to $100 \%$, and the "turn-on" region, which is the transition between the two previous regions. Steep turn-on curves correspond to good resolutions relative to offline by the trigger components, while high plateu levels reflect high reconstruction rates at the trigger-level. Once the efficiencies have been determined for individual trigger-components, hardware-level trigger decisions that involve combinations of multiple objects ${ }^{12}$ can be simulated by software that

\footnotetext{
${ }^{11}$ Offline refers to data that are collected by triggers and stored for inspection at the physicists' leisure, while online refers to data as they are being read out and inspected by the trigger hardware.

${ }^{12}$ For example, a muon at Level 3 consists of hits in the muon system scintillators along with hits in the muon drift chambers and a track reconstructed in the COT that extrapolates to pass within
} 
simply checks for the same logical combinations as required by the hardware triggers.

In measuring trigger efficiencies, it is important to avoid any bias that may arise from correlations between the trigger in question and the triggers used to collect the data sample with which the measurement will take place. For example, if we measure the efficiency for a trigger that has requirements A and B with data collected from triggers that required $\mathrm{B}$ and $\mathrm{C}$, then we are effectively measuring the efficiency of $\mathrm{A}$ because requirement B has already been satisfied. This will bias us towards higher trigger efficiencies because in general, the efficiency for A alone will be higher than the combined efficiency for A and B.

Data collected by jet triggers is used to measure the trigger efficiency of the "track" component of the lepton+track triggers[48, 49]. First, tau candidates are found offline and are required to pass most analysis-level cuts. The seed track $p_{T}$, tau-style track isolation and track multiplicity cuts are not applied because these quantities are later used to parameterize the trigger efficiency. The qualified offline tau candidates' seed tracks are matched to tracks made by the L1 tracking algorithm, the eXtremely Fast Tracker (XFT). The XFT efficiency is parameterized as a function of the tau's seed track $p_{T}$ and $\eta$, as well as by the number of tracks within $10^{\circ}$ of the seed track. The latter parameter accounts for possible variations in the XFT's track reconstruction efficiency on the density of hits in the COT (it's a weak dependence: 0.993 for a 1-prong tau, 0.988 for 2- and 3-prongs). The Lepton+Track triggers also have a tau-style track isolation requirement at L3. The isolation efficiency is parameterized as a function of the offline tau track isolation, the number of tracks within a $10^{\circ}$ cone about the seed track, and the maximal angle $\alpha_{t r k}$ between the seed track and another track associated with the tau ${ }^{13}$

The electron trigger efficiencies[50, 51] are determined from a sample of electrons $30 \mathrm{~cm}$ of the hits in the muon chambers.

${ }^{13}$ This parameter accounts for the difference in angular resolution between the offline and L3 algorithms. For example, a track with $p_{T}=2 \mathrm{GeV} / c$ that is determined to be at a $9.9^{\circ}$ angle to the seed track will be counted as a signal track offline. However, the same track may be determined to be at an $10.1^{\circ}$ angle to the seed track by the L3 algorithm, and thus be counted towards track isolation at the trigger level, causing the event to fail the trigger. 
from photon-conversions in muon- and jet-triggered data. The jet and muon triggers are considered to be uncorrelated with electron triggers. The lack of correlation between the electron and jet-triggers is not obvious, because both triggers require energy depositions in the calorimeter. The trigger used was a highly prescaled $8 \mathrm{GeV}$ calorimeter trigger. The $\mathrm{L} 1$ electron trigger requires $E_{T}>8 \mathrm{GeV}$ in a single EM tower of Emfr $>0.875$. Most events collected by the jet trigger have $8 \mathrm{GeV}$ distributed typically among a dozen towers with unrestricted EMFR. This sample, then, is not enriched with events that have $8 \mathrm{GeV}$ in a single EM tower, and can be used without fear of trigger-bias. On the other hand, a $50 \mathrm{GeV}$ jet trigger would cause bias, as the likelihood of a single tower in a $50 \mathrm{GeV}$ cluster having EM energy above $8 \mathrm{GeV}$ is non-negligible. Electrons from photon-conversions were used because electrons from $Z \rightarrow e e$ are typically too high in $p_{T}$ to probe the trigger's "turn-on" curve (Figure 4.11). An electron's trigger efficiency is parameterized by its calorimeter $E_{T}$ and track $p_{T}$. The plateu efficiencies for electrons are 0.98,1.00, and 0.99 for L1, L2, and L3 respectively.

In the $\mu \tau$ analysis, we require tight muons to have $p_{T}>20 \mathrm{GeV} / c$. We are safely in the plateu region of the trigger, where the inefficiency is due only to the reconstruction efficiencies of the XFT and muon trigger system scintillators at L1, and the muon drift chambers at L3. The parametrerization of the efficiencies is trivial: it amounts to a simple scale factor to be applied on muons in MC. The scale factor is 0.941 for muons that passed the CMUP trigger, and 0.987 for muons that passed the CMX trigger. These scale factors are found from $Z \rightarrow \mu \mu$ events in data[52]. For example, $Z \rightarrow \mu \mu$ events are found with one tight muon passing the CMX trigger, and the other muon traversing the fiducial region of the CMU and CMP detectors. The CMUP efficiency is then determined from the muon that passes through these detectors. The CMX efficiency is found using $Z \rightarrow \mu \mu$ collected by the CMUP trigger. 


\section{Trigger Simulation for MC Samples}

Once the trigger efficiencies have been measured and parameterized as functions of leptons' offline properties, it is a simple matter to apply these efficiencies to simulated leptons in MC samples. Suppose we have found that the L1 trigger efficiency for an electron depends on its $E_{T}$ as measured by the EM calorimeter, and its track $p_{T}$ from the COT. If we know that, for example, that for an identified electron of a given $E_{T}=E_{T}^{\prime}$ and $p_{T}=p_{T}^{\prime}$, the trigger efficiency at L1 is parameterized to be $\epsilon^{L 1}\left(E_{T}^{\prime}, p_{T}^{\prime}\right)=0.95$. We use a random number generator with a uniform distribution between zero and one to obtain a random number. The given electron will pass the L1 trigger requirement if the random number has a value below 0.95 , and will fail otherwise. We simulate L1, L2, and L3 trigger efficiencies in this manner for all identified leptons. An MC event will pass the simulated trigger if all trigger requirements are satisfied in the above manner.

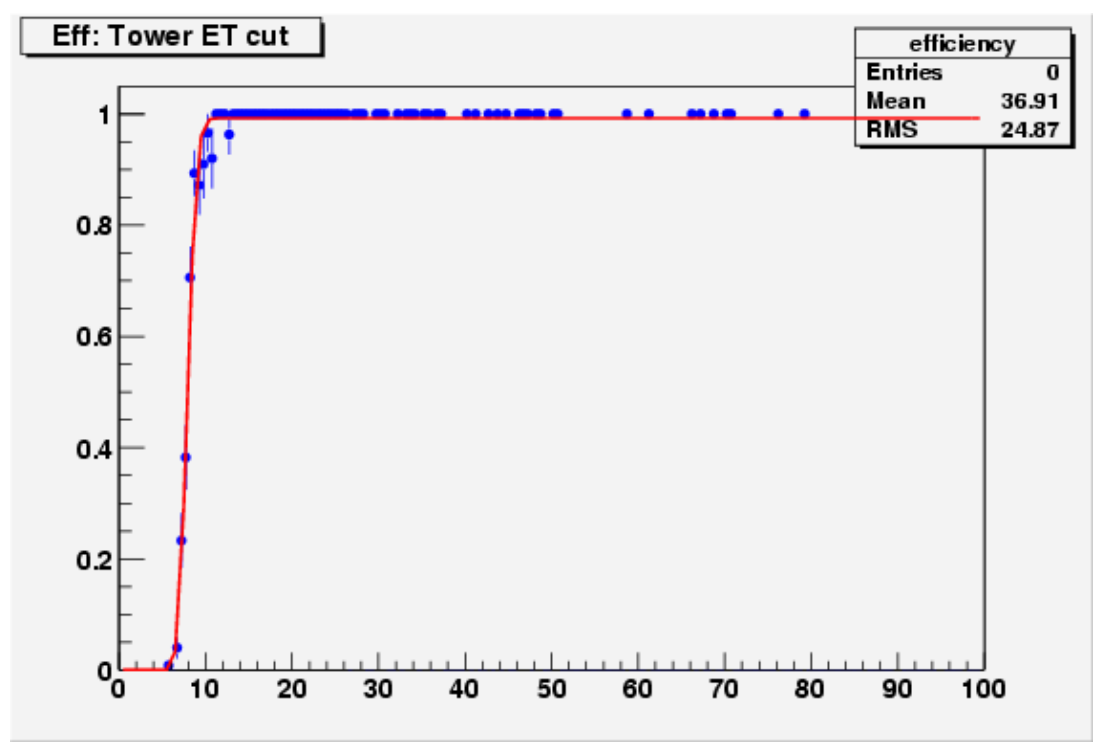

Figure 4.11: Efficiency of the Level 1 electron trigger vs offline electron $E_{T}$.

\subsubsection{Good Run Lists and Datasets}

A period of continuous data acquisition is called a "run". At CDF, runs can last from less than one hour to more than a day (the record run at CDF recorded 6.3 


\begin{tabular}{|c|c|}
\hline Trigger & Requirement \\
\hline \multicolumn{2}{|l|}{ Level 1} \\
\hline L1_CEM8_PT8 & $\begin{array}{l}\text { Seed Tower } E_{T}>8 \mathrm{GeV} \text { in CEM } \\
E_{\text {had }} / E_{\text {em }}<0.125 \text { if } E_{T}<16 \mathrm{GeV} \\
3 \text { or } 4 \text { layer XFT track w } / p_{T}>8 \mathrm{GeV} / c \\
\text { pointing to seed. }\end{array}$ \\
\hline \multicolumn{2}{|l|}{ Level 2} \\
\hline L2_CEM8_PT8 & $\begin{array}{l}\text { Cluster } E_{T}>8 \mathrm{GeV} \text { in CEM. } \\
\text { shoulder } E_{T}>7.5 \mathrm{GeV} \text {. } \\
E_{\text {had }} / E_{\text {em }}<0.125 \\
4 \text { layer XFT track } \mathrm{w} / p_{T}>8 \mathrm{GeV} / c \\
\text { pointing to seed. }\end{array}$ \\
\hline L2_CEM8_PT8_CES3 & $\begin{array}{l}\text { Same as L2_CEM8_PT8 } \\
\text { CES E > 3.0 GeV }\end{array}$ \\
\hline $\begin{array}{l}\text { L2_CEM8_PT8_CES3_ } \\
\text { TRK5_DPHI10_v-x }\end{array}$ & $\begin{array}{l}\text { Same as L2_CEM8_PT8_CES3 } \\
\text { Second } 4 \text { layer XFT track with } p_{T}>5.0 \mathrm{GeV} / c \\
\text { Angle between two tracks }>10^{\circ}\end{array}$ \\
\hline \multicolumn{2}{|l|}{ Level 3} \\
\hline \multicolumn{2}{|l|}{ L3_ELE8_TRK5_ISO } \\
\hline Event-Level & $\begin{array}{l}E_{T}>8 \mathrm{GeV}\left(z_{0}=0\right) \\
p_{T}>8 \mathrm{GeV} / c \\
|\Delta z|<8 \mathrm{~cm} \\
\chi_{\text {strip }}^{2}<20 \\
p_{T}>5.0 \mathrm{GeV} / c \\
|\eta|<1.5 \\
\text { No tracks w } / p_{T}>1.5 \mathrm{GeV} / c, \text { and }\left|\Delta z_{0}\right|<15 \mathrm{~cm} \\
\text { in } 0.175<\Delta R<0.524 \text { of "Iso Track" } \\
\left|z_{0}(e)-z_{0}(t r k)\right|<15 \mathrm{~cm} \\
\Delta R(e, \operatorname{trk})>0.175\end{array}$ \\
\hline
\end{tabular}

Table 4.8: Requirements of the electron+track trigger used to collect data for this analysis.

$\mathrm{pb}^{-1}$ of data.). The detector's performance is constantly monitored during datataking, and detailed descriptions are stored in a database. Although most of the time the detector is fully functional during data taking, there are rare occasions that require the temporary removal of a subdetector from the detector configuration. Not all components of the CDF are used in every analysis. For example, $B$ analyses rely heavily on silicon tracker, but may not need the SMX or the muon chambers at all, while a $H \rightarrow \tau \tau$ search would need the SMX and the muon chambers, but not 


\begin{tabular}{|l|l|}
\hline Trigger & Requirement \\
\hline \hline Level 1 & \\
\hline L1_CMUP8(CMX8)_PT8 & Stub w $/ P_{T}>6 \mathrm{GeV}$ in $\mathrm{CMUP}(\mathrm{CMX})$ \\
& 3 layer XFT track w $/ p_{T}>4.09 \mathrm{GeV} / c$ \\
& pointing to stub. \\
& (4-Layer XFT for the CMX-case) \\
\hline \hline Level 2 & \\
\hline L2_CMUP8_PT8: & 4 layer XFT track w $/ p_{T}>8 \mathrm{GeV} / c$ \\
& pointing to stub \\
L2_CMX8_PT8: & Auto Accept \\
\hline TRK5_DPHI10_V-X & Second 4 layer XFT track with $p_{T}>5.0 \mathrm{GeV} / c$ \\
& Angle between two tracks $>10^{\circ}$ \\
\hline \hline Level 3 & \\
\hline L3_CMUP(CMX)8_TRK5_ISO & \\
\hline Muon & \\
& $p_{T}>8 \mathrm{GeV} / c$ \\
& $|\Delta x|<20 / 15 \mathrm{~cm}(C M P / C M U)$ \\
& $|\Delta x|<30 \mathrm{~cm}(C M X)$ \\
& $p_{T}>5.0 \mathrm{GeV} / c$ \\
& $|\eta|<1.5$ \\
& No tracks w $/ p_{T}>1.5 \mathrm{GeV} / c$, and $\left|\Delta z_{0}\right|<15 \mathrm{~cm}$ \\
& in $0.175<\Delta R<0.524$ of "Iso Track" \\
& $\left|z_{0}(\mu)-z_{0}(t r k)\right|<15 \mathrm{~cm}$ \\
& $\Delta R(\mu, \operatorname{trk})>0.175$ \\
\hline \hline
\end{tabular}

Table 4.9: Requirements of the muon+track triggers used to collect data for this analysis.

the silicon detectors. It is important to exclude from consideration data that were recorded with detector configurations that are incompatible with one's analysis. One can define "good run lists" that can be used to exclude data collected during periods when necessary detector components were not operational. For the $e \tau$ analysis, we require the calorimeters, COT, and SMX to be operational. This results in $349 \pm$ $21 \mathrm{pb}^{-1}$ of data. For the $\mu \tau$ analysis, we also require that the CMX, CMU, and CMP muon chambers and associated trigger hardware were operational during data taking. The additional requirements result in a slightly smaller $\left(322 \pm 19 \mathrm{pb}^{-1}\right)$ dataset. The integrated luminosity is determined by the CLC (2.4.6). The error on the CLC acceptance is $4.2 \%[53]$, while the error on the total $p \bar{p}$ inelastic cross 


\begin{tabular}{|c|c|c|c|c|c|c|c|}
\hline & & \multicolumn{2}{|c|}{$H^{++} H^{--} \rightarrow e^{+} \tau^{+} e^{-} \tau^{-}$} & \multicolumn{3}{|c|}{$H^{++} H^{--} \rightarrow \mu^{+} \tau^{+} \mu^{-} \tau^{-}$} \\
\hline & & Flavor & $E_{T}$ & $|\eta|$ & Flavor & $E_{T}$ & $|\eta|$ \\
\hline \multirow{3}{*}{ 3-lepton } & $1^{\text {st }}$ lepton & $\mathrm{e}$ & 20 & $<1.0$ & $\mu$ & 20 & $<1.0$ \\
& $2^{\text {nd }}$ lepton & CdfTau $\left(e\right.$ or $\left.\tau_{h}\right)$ & 15 & $<1.0$ & CdfTau $\left(e\right.$ or $\left.\tau_{h}\right)$ & 15 & $<1.0$ \\
& $3^{\text {rd }}$ lepton & CdfTau $\left(e\right.$ or $\left.\tau_{h}\right)$ & 10 & $<1.3$ & ITS $\left(e, \mu\right.$, or $\left.\tau_{h}\right)$ & 10 & $<1.3$ \\
\hline \hline 4-lepton & $4^{\text {th }}$ lepton & ITS $\left(e, \mu\right.$ or $\left.\tau_{h}\right)$ & 10 & $<1.3$ & ITS $\left(e, \mu\right.$, or $\left.\tau_{h}\right)$ & 10 & $<1.3$ \\
\hline
\end{tabular}

Table 4.10: Lepton Requirements for $H^{++} H^{--} \rightarrow l^{+} \tau^{+} l^{-} \tau^{-}$signature.

section is $4 \%$ [54]. These errors are combined in quadrature and the resulting total uncertainty on the luminosity is $5.9 \%$.

\subsubsection{Three and Four Lepton Samples}

This analysis divides the events into two distinct categories: events that have at most three isolated leptons (3-lepton) and events that have four or more leptons (4-lepton). The lepton requirements for these two event-classes are listed in Table 4.10. There is a significant difference in the requirements of the third object: for the $\mu \tau$ search, the third object can an isolated track (ITS) that may correspond to a lepton of any flavor, while for the $e \tau$ search the third object must be a CdfTau (corresponding to an $e$ or $\tau_{h}$ ). The exclusive $e \tau$ decay modes heavily favor finalstates with electrons and hadronic taus, and the additional acceptance due to the inclusion of muons is not worth the associated increase in backgrounds.

\subsection{SM Backgrounds after Lepton ID}

We present a quick overview here to motivate our selection criteria for the 3-lepton and 4-lepton regions.

\section{- $\mathrm{W}+\mathrm{jets}$}

The cross section for inclusive $W \rightarrow l \nu_{l}$ production and decay is nearly $3 \mathrm{nb}$. The $W$ must be accompanied by at least two high $p_{T}$ jets that are misidentified as leptons (usually as hadronic taus or isolated track systems) to pass our lepton identification. This results in a reduction of at least $10^{5}$, and makes 
$W+$ jets a negligible background despite its large cross section. We use a PYTHIA MC sample to estimate the $\mathrm{W}+$ jets background, and account for any differences in the jet production rates, jet kinematics, and the jet $\rightarrow \tau_{h}$ misidentification rates in data and MC by assigning a scale factor to the MC.

\section{- Drell-Yan+jets}

The cross section for $Z^{*} / \gamma^{*} \rightarrow l l^{14}$, for $l$ being either $e$ or $\mu$, and for $m(l, l)>$ $30 \mathrm{GeV} / c^{2}$ is $\sim 330 \mathrm{pb}$. This background needs the associated production of at least one high- $p_{T}$ jet and its misidentification as a lepton (usually a CdfTau or ITS) to pass our lepton ID requirement. This results in a reduction of nearly $10^{3}$, making the "effective" $Z+\left(j e t \rightarrow \tau_{h}\right)$ cross section $\mathcal{O}(400 f b)$ - several times larger than our signal cross section. Drell-Yan+jets is our most significant background, but it is reducible to a large extent because it is possible to identify the $Z$ by finding two oppositely charged same-flavor leptons that make an invariant mass consistent with a $Z$. Furthermore, the total energy involved in the typical $Z+$ jets event is much less than that of an $\mathrm{H}^{++} \mathrm{H}^{--}$event, making further reductions possible.

\section{- Drell-Yan+direct photon}

The $Z^{*}+\gamma$ process can pass lepton identification if the photon is well-separated from the other two leptons in the event, and if it converts to an electronpositron pair that passes electron identification. There are two ways of getting a $Z+\gamma$ final state at CDF. The first involves a $Z$ in the s-channel decaying into leptons, followed by the radiation of a photon by the $Z$ s decay daughters. This process can be thought of as $q \bar{q} \rightarrow Z \rightarrow l^{+} l^{-} \gamma$. The second way of having $Z$ +photon production is direct $Z \gamma$ production in the t-channel. The first process is simulated by PYTHIA, while the second one is not. We used a MADGRAPH MC sample to investigate the contribution of the direct t-channel $Z \gamma$ production and found it to be negligible. Furthermore, $Z \rightarrow e e+\gamma$ events

\footnotetext{
${ }^{14}$ Henceforth it shall be implied that we include off-shell $Z$ s and photons when we use $Z$ and $\gamma$ unless a $Z$-mass resonance or final-state photon is implied by context.
} 


\begin{tabular}{|c|c|c|}
\hline Flavor Combination & $e \tau$ Sensitivity & $\mu \tau$ Sensitivity \\
\hline$e e+\left(j e t \rightarrow \tau_{h}\right)$ & 1 & 0 \\
\hline$\mu \mu+\left(j e t \rightarrow \tau_{h}\right)$ & 0 & 1 \\
\hline$e \mu+\left(j e t \rightarrow \tau_{h}\right)$ & 0 & 1 \\
\hline$\mu e+\left(j e t \rightarrow \tau_{h}\right)$ & 0 & 1 \\
\hline
\end{tabular}

Table 4.11: The flavor-dependent sensitivities of the two analyses to dilepton finalstates of $t \bar{t}$ decays.

are very easily removed with a selection described in the next section.

\section{- Top}

The $t \bar{t}$ cross section is $\sim 7 \mathrm{pb}$, and the final state almost always involves two $W \mathrm{~s}$ and two b-jets. This can result in a rich spectrum of signatures: Ignoring ISR/FSR, the final state can have either 6 jets, 1 lepton +4 jets, or 2 leptons +2 jets. The overall energy scale for $t \bar{t}$ events is very high, and many of the final-state particles will have very high transverse momenta. To pass lepton identification, the $t \bar{t}$ event would need either one $W$ to decay into an $e$ or $\mu$, and two of the jets to be misidentified as leptons, or both $W$ s to decay leptonically along with a jet misidentified as a lepton. The second scenario occurs more frequently due to the small lepton misidentification rates. We note that the $t \bar{t}$ contamination in the $\mu \tau$ analysis is roughly thrice that of the $e \tau$ analysis. This is due to the fact that the $\mu \tau$ search is sensitive to more lepton-flavor combinations of the $W$ decays, as shown in Table 4.11. The combination of $W \rightarrow l \nu_{l}$ branching ratios and jet $\rightarrow \tau_{h}$ misidentification rates result in a suppression of $\mathcal{O}\left(10^{-3}\right)$, making $t \bar{t}$ a negligible background in the $e \tau$ search, and a small background in the $\mu \tau$ search.

\section{- WW}

This process is totally negligible. $W W$ has a similar cross section to $t \bar{t}$, but lacks the two high $p_{T}$ b-jets.

\section{- WZ}

This process has a relatively small production cross section, but is significant 
because it can result in genuine trilepton final states. Due to the small jet $\rightarrow$ lepton misidentification rates, only the all-leptonic decays modes are significant sources of backgrounds. The $W Z$ production cross section is about $4 \mathrm{pb}$, and the full leptonic branching ratio is about $3 \%$, but, only certain flavor combinations are accepted by the $e \tau$ and $\mu \tau$ channels. After lepton identification, we expect about $0.8 \mathrm{WZ}$ events to remain in our data sample. The process is reducible by event-level kinematical selections.

\section{- $\mathrm{ZZ}$}

This process has a very small cross section, but can yield four leptons, and is kinematically most similar to our signal. Due to the small jet $\rightarrow$ lepton misidentification rate, only the all-leptonic decay modes are significant. The $Z Z$ production cross section is $\sim 2 \mathrm{pb}$, but the branching ratio to 4-lepton final states is less than $1 \%$. In our data sample, we expect about $0.3 Z Z$ events after lepton identification. Kinematical cuts at the event-level can reduce this background by a factor of three. We note that this process is the only significant background in the 4-lepton region.

\section{- QCD}

The production cross section multi $(\geq 3)$ jet events is many orders of magnitude larger than our signal's. However, three misidentification rates are needed: $10^{-4+-2+-2}=10^{-8}$. Furthermore, only a small fraction of the misidentified leptons form jets will have $p_{T}>20$. These factors make QCD a negligible background.

\section{- Cosmic Rays}

Cosmic ray muons can be a source of background if they pass sufficiently close to the luminous region in space and time ( i.e $\left|Z_{0}\right|<60 \mathrm{~cm}, d_{0}<1 \mathrm{~cm}$, $\Delta t($ cosmic, event $)<\sim 100 \mathrm{~ns})$. The significance of cosmic ray backgrounds depends on the analysis signature, and also on the average instantaneous luminosity during data-taking. Due to CDF's effective rejection of cosmic rays, as 


\begin{tabular}{|c|c|c|c|c|c|c|}
\hline \hline \multicolumn{7}{|c|}{$\left.H^{++} H^{--} \rightarrow e^{+} \tau^{+} e^{-} \tau^{-} \mathbf{( 1 1 0 ~ G e V} / c^{2}\right)$} \\
\hline \hline Signal & $Z Z$ & $Z W$ & $Z \rightarrow e e$ & $Z \rightarrow \tau \tau$ & $t \bar{t}$ & $W \rightarrow e \nu_{e}$ \\
\hline $4.68 \pm 0.06$ & $0.29 \pm 0.03$ & $0.52 \pm 0.07$ & $33.3 \pm 1.2$ & $1.25 \pm 0.26$ & $0.09 \pm 0.03$ & $1.17 \pm 0.44$ \\
\hline \hline \multicolumn{7}{|c|}{$\left.H^{++} H^{--} \rightarrow \mu^{+} \tau^{+} \mu^{-} \tau^{-} \mathbf{( 1 1 0 ~ G e V} / c^{2}\right)$} \\
\hline Signal & $Z Z$ & $Z W$ & $Z \rightarrow \mu \mu$ & $Z \rightarrow \tau \tau$ & $t \bar{t}$ & $W \rightarrow \mu \nu_{\mu}$ \\
\hline $4.71 \pm 0.06$ & $0.36 \pm 0.03$ & $0.72 \pm 0.08$ & $25 \pm 1.4$ & $2.8 \pm 0.4$ & $0.29 \pm 0.03$ & $1.02 \pm 0.28$ \\
\hline
\end{tabular}

Table 4.12: Expected signal and backgrounds with at least three identified leptons the $e \tau$ and $\mu \tau$ searches.

described in section 2.4.3 and also to the multilepton nature of our signature, cosmic rays are an insignificant background for our search.

We next discuss event-level cuts that further reduce the above backgrounds. For reference, the signal and background levels for the two searches after lepton identification and before separation into 3-lepton and 4-lepton samples, are summarized in Table 4.12.

\subsection{Event-Level Selections}

The SM background levels for the $e \tau$ or $\mu \tau$ searches after trigger and lepton selections are about 35 events. The expected signal is near the level of statistical fluctuations associated with the backgrounds. It is necessary to make further selections to increase the significance of the signal in the final data set. The goal is to reject as many backgrounds as possible at the smallest cost in terms of signal acceptance. This process usually involves the exploitation of kinematical peculiarities of the signal and various background processes. The quantity $S / \sqrt{S+B}$, where $S$ stands for signal and $B$ for backgrounds serves as a rough guide for the optimization of selections. We choose, out of several candidates, those event-level requirements that maximize $S / \sqrt{S+B}$. 


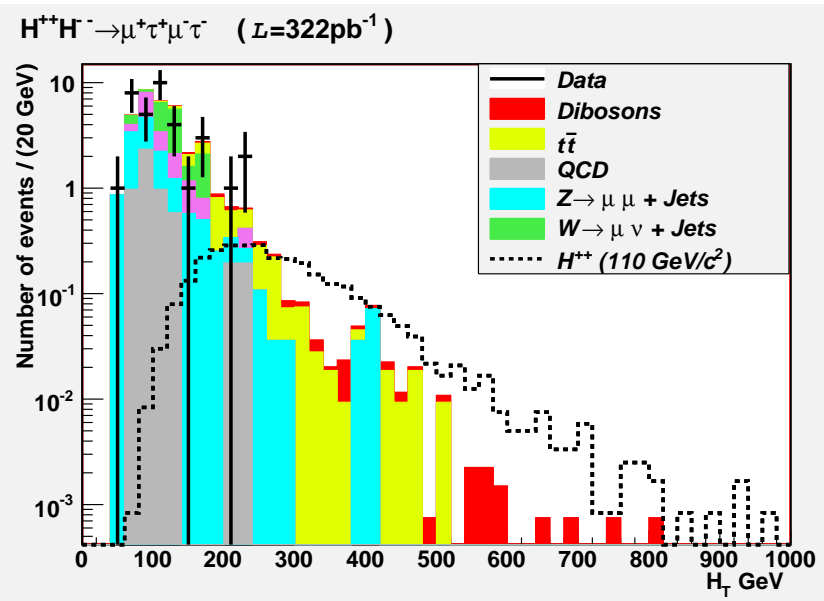

Figure 4.12: The $H_{T}$ distribution for signal and backgrounds after lepton identification in the $\mu \tau$ search. The data are plotted with statistical errors.

\subsubsection{Event Selections for Three-Lepton Events}

- $\mathbf{H}_{\mathrm{T}}$

At the Tevatron, the $H^{ \pm \pm}$are pair produced at high $p_{T}\left(H^{ \pm \pm}\right)$, and the total energy in the production $\mathrm{CM}$ frame is very large compared to that of the typical $Z+$ jet, $W+$ jet, and diboson events. We can take advantage of this by constructing the total transverse energy, called $H_{T}$, as the scalar sum of the $\mathscr{H}_{T}$ and the lepton $E_{T} \mathrm{~s}^{\prime}$ :

$$
H_{T} \equiv \mathscr{H}_{T}+\Sigma E_{T}^{\text {leptons }} .
$$

The $H_{T}$ distributions for signal and backgrounds for all events that pass lepton identification are shown for the $\mu \tau$ and $e \tau$ searches in Figures 4.12 and 4.13 respectively.

\section{- Drell-Yan Removal}

To suppress Drell-Yan, $Z Z$, and $Z W$ backgrounds, events that have two oppositely charged leptons in certain mass ranges are either removed from consideration, or are required to pass additional requirements. Low mass Drell-Yan 


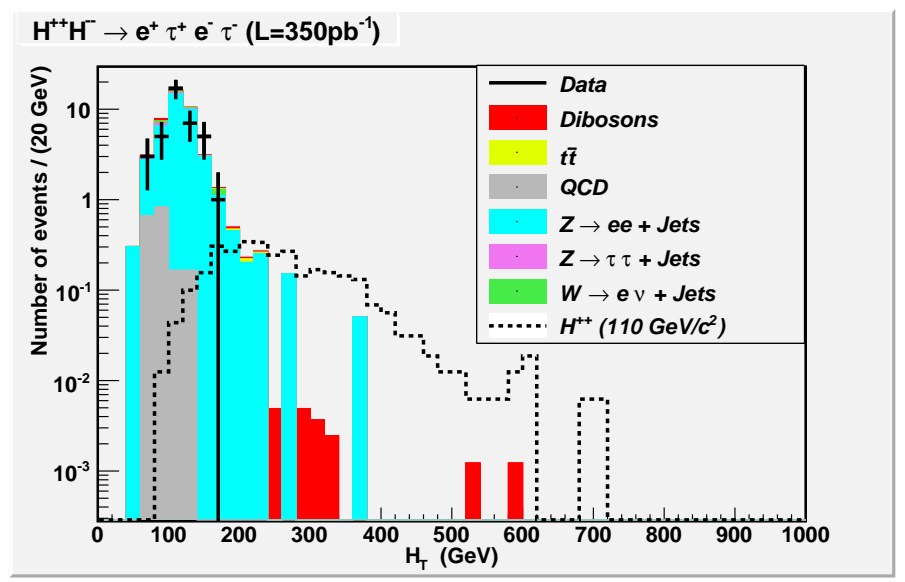

Figure 4.13: The $H_{T}$ distribution for signal and backgrounds after lepton identification in the $e \tau$ search. The data are plotted with statistical errors.

backgrounds are removed by stipulating that the minimum invariant mass for a pair of oppositely charged leptons must be $30 \mathrm{GeV} / c^{2}$. To remove $Z$-based backgrounds, events with two oppositely charged electrons (we consider all pairs that include the leading electron and any other particle with Emfr > 0.9) that make an invariant mass in the range $71 \mathrm{GeV} / c^{2}<\mathrm{M}\left(e^{+} e^{-}\right)<111 \mathrm{GeV} / c^{2}$ are required to have $H_{T}>300 \mathrm{GeV}$. The $H_{T}$ requirement retains some signal that accidentally falls in the mass range, but effectively removes $Z \rightarrow e e$, and to a lesser extent, $Z W$ and $Z Z$ backgrounds. For the case of $Z \rightarrow \mu \mu$, events with oppositely charged muons (we consider all paris that include the lead muon) that make an invariant mass in the range $66 \mathrm{GeV} / c^{2}<\mathrm{M}\left(\mu^{+} \mu^{-}\right)<$ $116 \mathrm{GeV} / c^{2}$ are required to have $H_{T}>350 \mathrm{GeV}$. The wider mass range and higher $H_{T}$ requirement reflect the fact that the $H_{T}$ distribution is more sensitive to a muon's $p_{T}$ than it is to an electron's $p_{T}$, because the $\mathbb{F}_{T}$ corrections for muons are relatively large. For example, consider a $Z \rightarrow \mu \mu$ event with the leading muon having $p_{T}=60 \mathrm{GeV} / c$, and $E_{T}=3 \mathrm{GeV}$ in the calorimeter. If the muon's track $p_{T}$ is mismeasured as $80 \mathrm{GeV} / c$, then the corresponding correction to the $\mathbb{F}_{T}$ is likely to result in a corrected $\mathbb{F}_{T}$ that is $\sim 20 \mathrm{GeV}$ too large. The $H_{T}$, then, is overestimated by $40 \mathrm{GeV}$, making the event more 


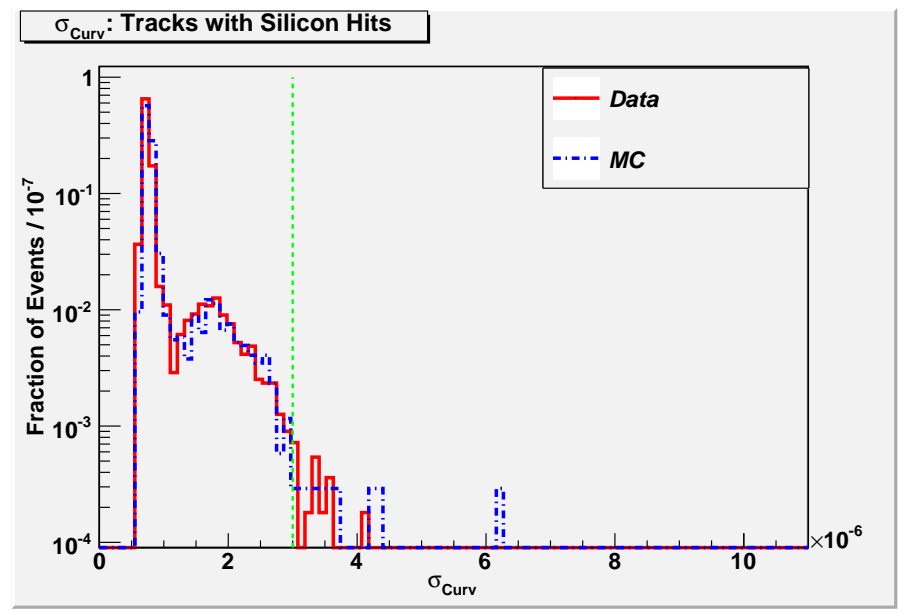

Figure 4.14: The error on the track curvature for tracks with silicon hits. All tracks that fall to the right of the cut-line are deemed poorly measured, and rejected. Data ( $\mathrm{Z} \rightarrow$ ee events with mass reconstructed by EM calorimeter) are plotted in solid red, and MC events are plotted with the dashed blue line.

likely to pass this selection. If the mismeasurement is small enough that the invariant mass of the two muons remains in the range $66-116 \mathrm{GeV} / c^{2}$, then the $350 \mathrm{GeV} H_{T}$ requirement effectively removes the $Z \rightarrow \mu \mu$ background. If, on the other hand, the muon's track is grossly mismeasured, then the event is likely to pass both the $H_{T}$ and $Z$-removal cuts. Fortunately, we can reduce the probability of overestimating a $Z \rightarrow \mu \mu$ muon's $p_{T}$ by $20 \mathrm{GeV} / c$ to less than 1 per mil, as described below.

\section{- $\sigma_{C u r v}$ for Leading Track}

In the $\mu \tau$ search, we guard against falsely large $H_{T}$ resultant from mismeasured muon $p_{T} \mathrm{~s}$ by placing additional requirements on the highest $p_{T}$ track in the event. The estimated uncertainty on a track's curvature is obtained from the covariance matrix of the tracking algorithm's fit to the track helix. We require the error to be less than $7 \times 10^{-6}$, and less than $3 \times 10^{-6}$ if the track has at least three hits in axial layers of the silicon detector. The curvature error, $\sigma_{\text {curv }}$, is plotted for tracks that have at least three axial silicon hits in Figure 4.14. For tracks that do not have silicon hits, $\sigma_{\text {curv }}$ is plotted in Figure 4.15. 


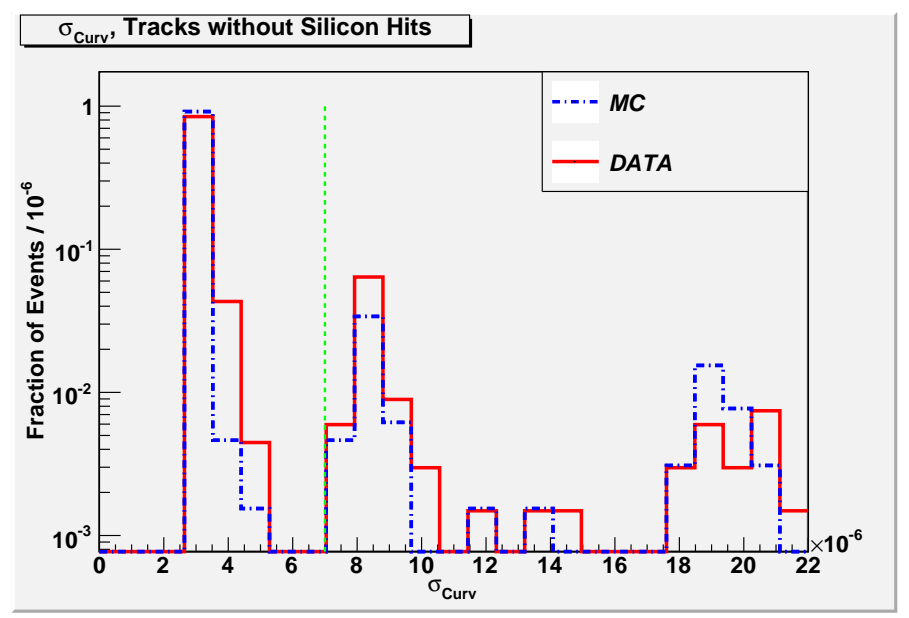

Figure 4.15: The error on the track curvature for tracks without silicon hits. All tracks that fall to the right of the cut-line are deemed poorly measured, and rejected. Data ( $\mathrm{Z} \rightarrow$ ee events with mass reconstructed by EM calorimeter) are plotted in solid red, and MC events are plotted with the dashed blue line.

\section{- $Z \rightarrow e e+\gamma$ Removal}

As mentioned, the $Z \rightarrow e e+\gamma$ process is accounted for by the inclusive $Z \rightarrow$ ee PYTHIA MC estimation. We have devised a special selection to remove $Z \rightarrow e e+\gamma$ events, not because they constitute a significant background, but simply because we can remove them in a manner that is $100 \%$ efficient for our signal. We require events that are consistent with having three electrons to also have $20 \mathrm{GeV}$ of $\mathbb{E}_{T}$. The signal efficiency is very high because decays of the high- $p_{T}$ taus result in $\mathbb{F}_{T}$. On the other hand, most $Z \rightarrow e e+\gamma$ events will fail this requirement because there are no neutrinos in the event, and only a small amount of $\mathbb{F}_{T}$ will usually result from detector effects.

\section{- Like-Sign Mass}

We require the invariant mass of any two like-sign leptons be in the range $30 \mathrm{GeV} / c^{2}<M_{L S}<125 \mathrm{GeV} / c^{2}$. Figure 4.16 shows this mass distribution for signal $\left(110 \mathrm{GeV} / \mathrm{c}^{2}\right)$ and backgrounds that remain after lepton identification in the $\mu \tau$ search. Given our analysis' acceptance and the signal cross section, even in the absence of all backgrounds we would expect mass limits of $~$ 


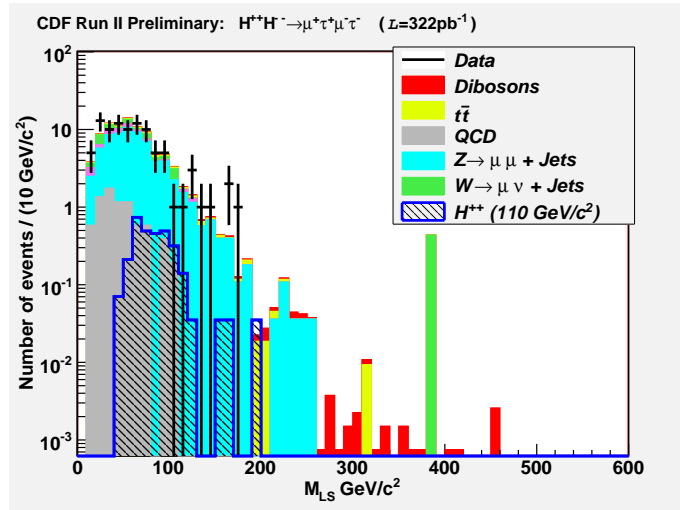

Figure 4.16: The invariant mass of like-sign leptons for signal and backgrounds. The mass window $30 \mathrm{GeV} / c^{2}<M_{L S}<125 \mathrm{GeV} / c^{2}$ is very efficient for $100 \mathrm{GeV} / c^{2}<$ $M_{H^{ \pm \pm}}<120 \mathrm{GeV} / c^{2}$

$115 \mathrm{GeV} / c^{215}$. We chose the upper bound of $125 \mathrm{GeV} / c^{2}$ to retain nearly $100 \%$ efficiency for signal, yet reduce backgrounds that might otherwise pass the $H_{T}$ requirement.

\section{- Cosmic Removal}

Cosmic ray muons are removed by requiring a pair of muons be consistent with particles that originate from primary interaction as explained in section 2.4.3. The TOF is the most powerful tool for cosmic ray removal. The timedifference between the two "legs" is plotted for $Z \rightarrow e e$ and tagged cosmic ray events in Figure 4.17.

\subsubsection{Event-Level Selections for Four-Lepton Events}

\section{- Cosmic Removal}

Cosmic ray muons are removed with the same cosmic ray tagger used for the three-lepton events.

\footnotetext{
${ }^{15}$ Technically, we should drop this requirement, recalculate our limits with the assumption of zero observed events in the signal region, and use the mass reach as a guide for the upper limit on the mass range. In the mass range of interest, however, this cut is $\sim 99 \%$ efficient for signal and thus does not affect our mass reach by more than a few $\mathrm{MeV} / \mathrm{c}^{2}$.
} 


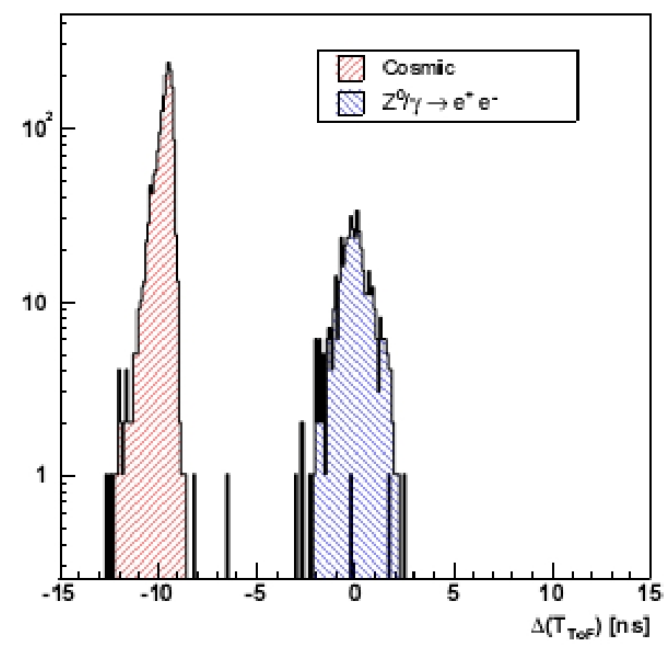

Figure 4.17: The time difference as measured by the TOF for two muon-candidates tagged as cosmic rays, and for two electrons from $Z \rightarrow e e$ events. The TOF enables unambiguous tagging of cosmic ray events.

\section{- $\mathrm{H}_{T}$}

For the $e \tau$ search, the $H_{T}$ must be greater than $100 \mathrm{GeV}$, and for the $\mu \tau$ search the $H_{T}$ must be greater than $120 \mathrm{GeV}$. The stricter requirement for the $\mu \tau$ search protects against spurious $H_{T}$ that can result from the relatively large $\mathbb{H}_{T}$ corrections associated with muons.

\section{- Drell-Yan Removal}

For the $e \tau$ search, if the highest invariant mass constructed from two oppositely charged electrons is less than $120 \mathrm{GeV} / c^{2}$, we require the $\mathbb{E}_{T}$ to be greater than $20 \mathrm{GeV}$. Also, as with the 3-lepton events, if the event is consistent with having four electrons, we require at least $20 \mathrm{GeVof} \mathbb{F}_{T}$. The EM fraction of the lowest-Emfr lepton is plotted for signal and $Z Z$ events in Figure 4.18. For the $\mu \tau$ search, we require $H_{T}>150 \mathrm{GeV}$ if the invariant mass of two oppositely charged muons (or a muon and a track) falls in the range $66 \mathrm{GeV} / c^{2}<M_{O S}<$ $116 \mathrm{GeV} / c^{2}$. 


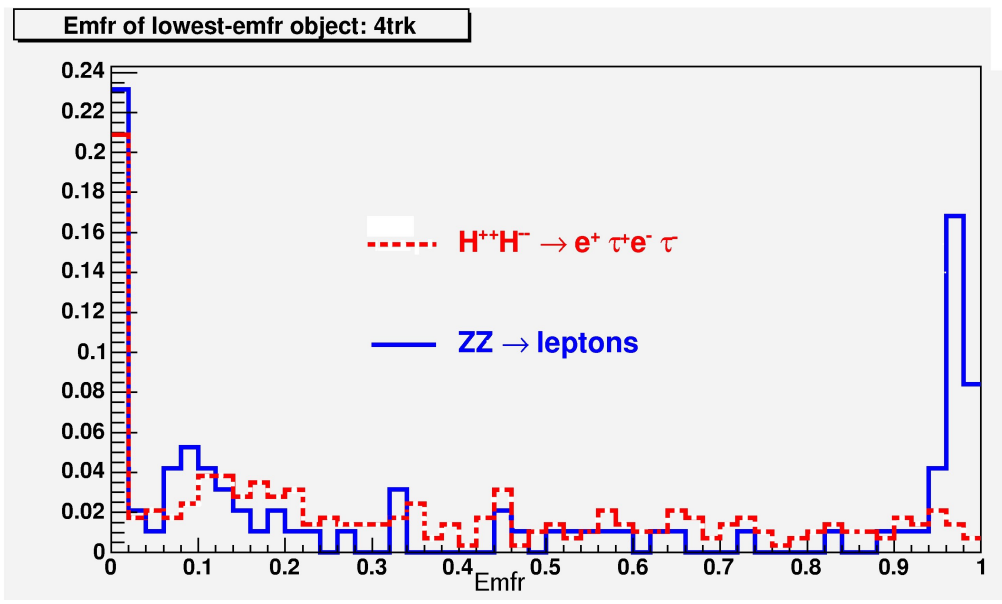

Figure 4.18: The EM fraction of the calorimeter energy cluster of the lowest Emfr lepton in the 4-lepton region of the $e \tau$ search. The signal is plotted in dashed red and the $Z Z$ background is plotted in solid blue. Both samples were created with the PYTHIA generator and run through the same detector simulation. Both histograms are normalized to unit area. 


\section{Chapter 5}

\section{Background Determination}

In this section we discuss the estimation of Standard Model backgrounds to our signal. We use data to estimate QCD, photon+jets, and cosmic ray backgrounds. Other backgrounds are estimated using Monte Carlo simulations. Without looking in the signal region, we study our background predictions in several control samples to validate, and, if necessary, assign systematic uncertainties and/or modify, our background predictions.

\subsection{Monte Carlo Samples}

The Monte Carlo samples used to determine signal and background acceptancees are summarized in Table 5.1. Processes with large cross sections, such as QCD, $\gamma+$ jets, $W+$ jets, and $Z+$ jets, are determined from and/or extensively checked in data. The processes with smaller cross sections, such as dibosons and top, are more difficult to check with data, but are likely to be well-simulated by the MC generators. These backgrounds mimic our signal through decay-products of the "hard subprocess" (the inelastic $2 \rightarrow 2$ interaction), which is considered to be well-simulated compared to the ISR/FSR implementation of associated jet production. 


\begin{tabular}{|l|l|l|l|l|}
\hline MC Process & Generator & NeV & $\boldsymbol{\sigma} \times \boldsymbol{B R}(\boldsymbol{p b})$ & $\mathcal{L}\left(\boldsymbol{f b}^{\mathbf{- 1}}\right)$ \\
\hline \hline$Z / \gamma^{*} \rightarrow e e$ & PYTHIA & $2,255,968$ & 326 & 6.92 \\
$Z / \gamma^{*} \rightarrow \tau \tau$ & PYTHIA & $1,062,031$ & 477 & 2.23 \\
$Z \gamma^{*} \rightarrow e e \gamma$ & ALPGEN & 211,682 & 12 & 17.6 \\
$Z / \gamma^{*} \rightarrow \mu \mu$ & PYTHIA & $3,252,332$ & 490 & 6.6 \\
$W \rightarrow e \nu_{e}$ & PYTHIA & $2,303,706$ & 2777 & 0.83 \\
$W \rightarrow \mu \nu \mu$ & PYTHIA & $2,624.054$ & 2777 & 0.9 \\
$t \bar{t}$ & PYTHIA & 231,158 & 6.7 & 34.5 \\
$Z Z^{*} \rightarrow$ leptons & PYTHIA & 10,000 & 0.0803 & 124.5 \\
$W Z^{*} \rightarrow l e p t o n s$ & MADGRAPH & 120,000 & 0.278 & 421.7 \\
Signal $(e \tau), 90 \mathrm{GeV} / c^{2}$ & PYTHIA & 10,000 & 0.199 & 50.3 \\
Signal $(e \tau), 100 \mathrm{GeV} / c^{2}$ & PYTHIA & 30,000 & 0.126 & 237 \\
Signal $(e \tau), 110 \mathrm{GeV} / c^{2}$ & PYTHIA & 46,000 & 0.0894 & 514.5 \\
Signal $(e \tau), 120 \mathrm{GeV} / c^{2}$ & PYTHIA & 30,000 & 0.0628 & 477.8 \\
Signal $(e \tau), 130 \mathrm{GeV} / c^{2}$ & PYTHIA & 10,000 & 0.0425 & 110 \\
Signal $(\mu \tau), 90 \mathrm{GeV} / c^{2}$ & PYTHIA & 10,000 & 0.199 & 50.3 \\
Signal $(\mu \tau), 100 \mathrm{GeV} / c^{2}$ & PYTHIA & 30,000 & 0.126 & 237 \\
Signal $(\mu \tau), 110 \mathrm{GeV} / c^{2}$ & PYTHIA & 46,000 & 0.0894 & 514.5 \\
Signal $(\mu \tau), 120 \mathrm{GeV} / c^{2}$ & PYTHIA & 30,000 & 0.0628 & 477.8 \\
Signal $(\mu \tau), 130 \mathrm{GeV} / c^{2}$ & PYTHIA & 10,000 & 0.0425 & 110 \\
\hline
\end{tabular}

Table 5.1: MC samples used to study signal and background processes.

\subsection{Blind Analysis}

To minimize the risk of experimental bias, we avoid any data samples that have bearing on the presence of signal until all selections have been finalized. As we look at data to validate our background estimations, we must restrict ourselves to "control regions", where we can work without sensitivity to our signal. To remain "blind" to the presence of signal, we inspect data samples only if the predicted amounts of signal are insignificant compared to the predicted background levels. In some cases, it suffices to increase backgrounds by relaxing some selections such as the lepton track isolation or track multiplicity requirements. In other cases, we must suppress the potential signal by reversing one or more analysis selections. For example, we can look at events that pass all selections except that they have $H_{T}<150 \mathrm{GeV}$ instead of $H_{T}>190 \mathrm{GeV}$. Or, if we want to look at the high- $H_{T}$ region, we can reverse the track isolation $($ TIso $>2 \mathrm{GeV} / c$ ) for one of the leptons. By reversing a 


\begin{tabular}{|c|c|c|c|}
\hline \hline \multicolumn{4}{|c|}{$\boldsymbol{e} \boldsymbol{\tau}$ Background Predictions } \\
\hline \hline Process & Method & NEv in 3-Lepton & NEv in 4-Lepton \\
\hline$Z+$ jets & PYTHIA MC & $0.16 \pm 0.15$ & $0_{-0.0}^{+0.05}$ \\
\hline Dibosons & PYTHIA / MADGRAPH MC & $0.06 \pm 0.01$ & $0.04 \pm 0.01$ \\
\hline$t \bar{t}$ & PYTHIA MC & $0.01 \pm 0.01$ & $0_{-0.0}^{+0.01}$ \\
\hline$W \rightarrow e \nu_{e}+$ jets & PYTHIA MC & $0.0_{-0.0}^{+0.18}$ & $0.0_{-0.0}^{+0.01}$ \\
\hline $\mathrm{QCD} / \gamma+$ jets & Data & $0.001 \pm 0.001$ & $0 !$ \\
\hline \hline Total & & $\mathbf{0 . 2 3}_{-\mathbf{0 . 1 6}}^{+0.26}$ & $\mathbf{0 . 0 4}+\mathbf{0 . 0 4}$ \\
\hline
\end{tabular}

Table 5.2: Background estimations for the e $\tau$ search. Both statistical and systematic errors are included in quadrature.

selection, the resulting sample excludes the signal region by definition, but we still make our signal and background estimations first to ensure that the resulting data sample is predicted to be sufficiently devoid of signal.

\subsection{Backgrounds and Control Regions for the $e \tau$ Search}

Our background estimations for the $e \tau$ search are summarized in Table 5.2. The methods used to obtain and validate these predictions are described below.

\subsubsection{QCD and Photon+jets Backgrounds}

The QCD and Photon+jets backgrounds are completely negligible. Their determination is important only for consistency in various control regions rather than in the signal region. These backgrounds are determined using two different methods, and their results are compared in relevant control regions. Both methods exploit the disparity between the electron track-isolation distributions of primary leptons and fake or misbegotten leptons that are members of hadronic jets.

\section{QCD Background Determination}

Backgrounds that supply electron candidates within jets are estimated using track isolation extrapolation. As we have seen in Figure 4.7, the track isolation distribution for fake leptons from QCD can be well-approximated by a first-degree polynomial. One simply fits the distribution in a region that is uncontaminated 
by lepton-bearing electroweak processes (i.e. $4 \mathrm{GeV} / c<$ TIso $<12 \mathrm{GeV} / c$ ) and extrapolates the line to the "signal" region TIso $<2 \mathrm{GeV} /$ c. This accounts for $\pi^{0}$ conversions, semileptonic decays, and charged pions that fake electrons through either charge exchange or through an overlap with a $\pi^{0}$ that provides a good EM cluster.

\section{Gamma+jet Background Determination}

In general, photon+jets backgrounds cannot be accurately determined by simply extrapolating along TIso. Asymmetric photon conversions (e.g. the softer track is soft: $p_{T}<1 \mathrm{GeV} / c$ ) are the dominant reason for conversion tagging inefficiency. As a result, untagged conversion electrons are not evenly distributed along TIso, but favor the signal $($ TIso $<2 \mathrm{GeV} / c)$ region. However, if one does not remove conversions, then the tagged conversions in the extrapolation region $(4 \mathrm{GeV} / c<$ TIso $<12 \mathrm{GeV} /$ c) may account for the untagged conversions in the signal region, but the accuracy will generally depend on the choice of signal and extrapolation regions.

We determine the total number of conversion-electrons in a given data sample by dividing the number of tagged conversions by the conversion tagging efficiency ${ }^{1}$. The conversions in the signal region that are due to QCD (i.e. photons from $\pi^{0} \mathrm{~s}$ ) are determined by extrapolating along the TIso of the converting photon. All QCDbased conversions are subtracted from the total number of conversions, leaving us with the $\gamma+$ jets component. We then create a TIso distribution for the conversionelectrons by using data to provide the component of TIso that is due to the underlying event, and $\mathrm{MC}$ to provide the component that is due to the conversion process alone. The number of events with TIso $<2 \mathrm{GeV} / c$ amounts to the predicted $\gamma+$ jets background.

The above method has the advantage that MC is used only to simulate the component of TIso that comes from the photon-conversion process, namely, the

\footnotetext{
${ }^{1}$ We summarize one method that is used to obtain conversion-tagging efficiencies from data[55] in the appendix.
} 


\begin{tabular}{|c|c|c|}
\hline Background & Properties & Formula \\
\hline$\gamma+$ jets & Conv, TIso $_{\gamma}<2$ & 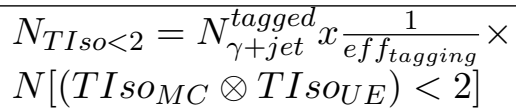 \\
\hline$\pi^{ \pm}$based QCD & TIsole $>2$ & $N_{\text {TIso }<2}=\frac{1}{4} \times N_{4<\text { TIso }<12}$ \\
\hline$\pi^{0}$ based QCD & Conv, TIso $_{\gamma}>2$ & $N_{\text {TIso }<2}=\frac{1}{4} \times N_{4<\text { TIso }<12}$ \\
\hline
\end{tabular}

Table 5.3: Outline of algorithms used to estimate QCD $/ \gamma+$ jets backgrounds. Note that $T I$ so $_{U E}$ is obtained from $Z \rightarrow e e$ events, and the $\otimes$ represents convolution.

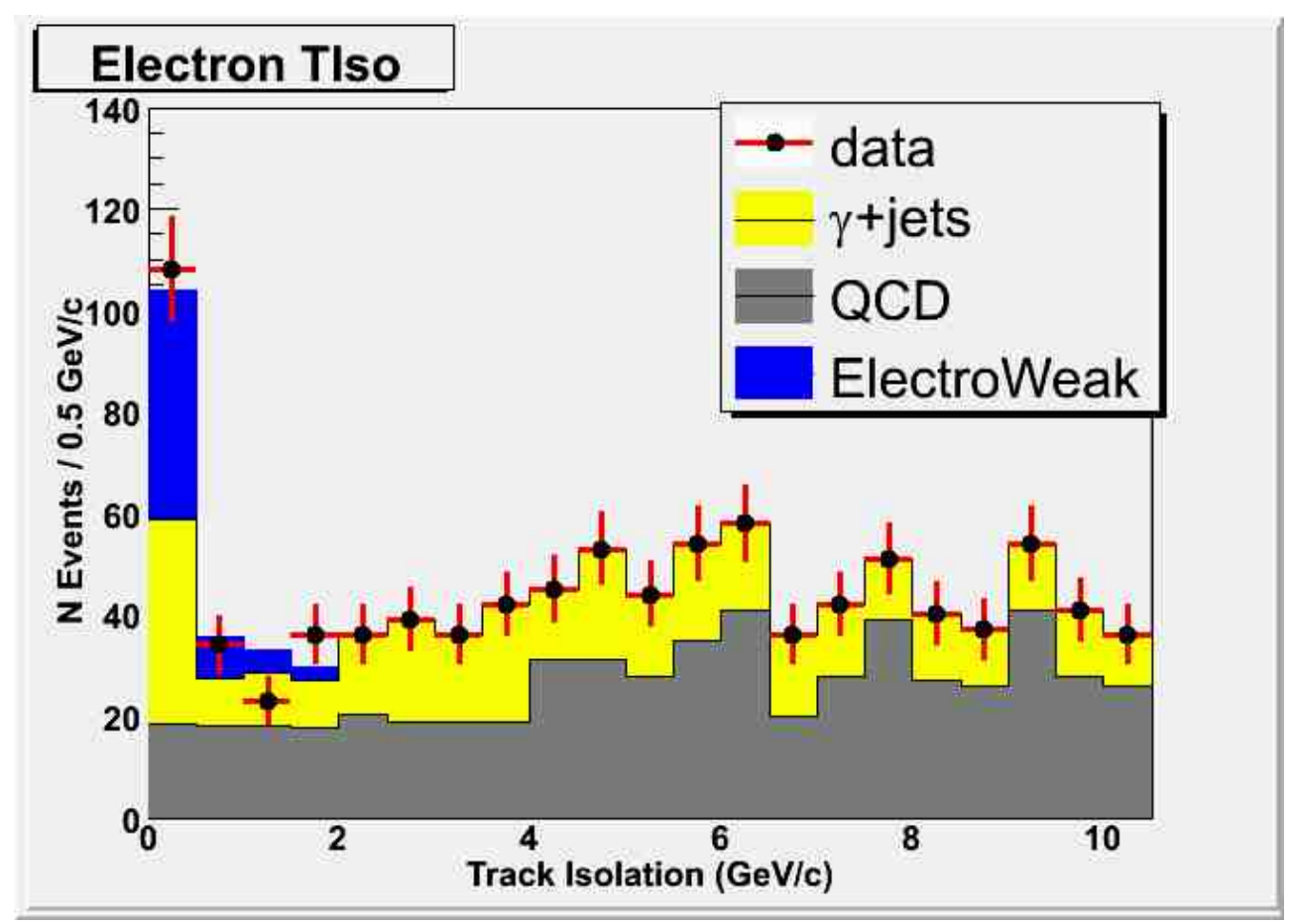

Figure 5.1: Electron TIso distribution for a $\mathrm{QCD} / \gamma+$ jets-enriched region. The numbers of events in the first four bins are predicted by our QCD (gray) and $\gamma+$ jets (yellow) estimation methods, and Monte Carlo for electroweak and top backgrounds (blue). The $\gamma+$ jets background peaks in the first bin, corresponding to the near total inefficiency of track reconstruction for tracks of $p_{T}<500 \mathrm{MeV} / c$.

conversion partner's $p_{T}$, while the component of TIso that is due to the underlying event is taken directly from data. The method is summarized in Table 5.3. Figure 5.1 shows the electron TIso distribution for a $\mathrm{QCD} / \gamma+$ jets-enriched sample, the large $\gamma+$ jets component in the first bin corresponds to the nearly total inefficiency of track reconstruction for particles with $p_{T}<500 \mathrm{MeV} / c$. 


\section{Validation of QCD and Photon+jets Predictions}

To validate our estimation method, we check our QCD and $\gamma+$ jets predictions in various control regions - as long as there is a sizable $\mathrm{QCD} / \gamma+$ jets presence, we can check for consistency by counting the number of events with TIso ${ }^{\text {ele }}<2 \mathrm{GeV} / c$. We also create a dedicated control sample that is enriched with QCD and $\gamma+$ jets by starting with "loose lepton selections" (Table 5.4) and removing Drell-Yan events by requiring that there be only one tight electron per event. We also remove $W\left(\rightarrow e \nu_{e}+j e t s\right)$ events by requiring that $\mathbb{F}_{T}<30 \mathrm{GeV}$. The kinematical distributions for $\gamma+$ jets are obtained from the tagged photon conversions with TIso ${ }^{\gamma}<2 \mathrm{GeV} / c$, and scaled down to the amount expected in the $T I s o^{e}<2 \mathrm{GeV} / c$ region. Likewise, for QCD, a given distribution is obtained from both tagged photon (from $\pi^{0} \mathrm{~s}$ ) conversions with TIso $^{\gamma}>2 \mathrm{GeV} / c$ and also the non-conversion sample $\left(\right.$ TIso $\left.{ }^{e}>2 \mathrm{GeV} / c\right)$, and scaled down to the amount expected in the TIso ${ }^{e}<2 \mathrm{GeV} / c$ region. The kinematical distributions in all control regions for the QCD and $\gamma+$ jets backgrounds are obtained in this fashion. We plot various kinematical distributions and the QCD/Photon+jets-enriched region along with our predictions in Figure 5.2.

\section{Increasing Statistical Precision}

The QCD, $\gamma+$ jets, and $W+$ jets backgrounds are so heavily suppressed that no events are predicted to pass all analysis cuts. Typically, the associated statistical error would be the "weight" of one event. From Table 5.1 we can see that the $W \rightarrow e \nu$ sample, for example, corresponds only to $0.83 \mathrm{fb}$, or about 2.5 times our data sample. The weight of an event in MC sample then is about 0.4, and sets the level of statistical uncertainty associated with the determination of this background from MC. To obtain larger samples we relax the track isolation requirement on the tau and LTC to $10 \mathrm{GeV} / c$, and also relax the track multiplicity requirements to accept 2-prong and 4-prong taus and LTCs as well as the usual 1- and 3-prongs. We must be sure that the kinematical quantities of interest (i.e. $H_{T}$ ) are not correlated with the lepton-identification cuts that are relaxed. The $H_{T}$ distribution is independent of 

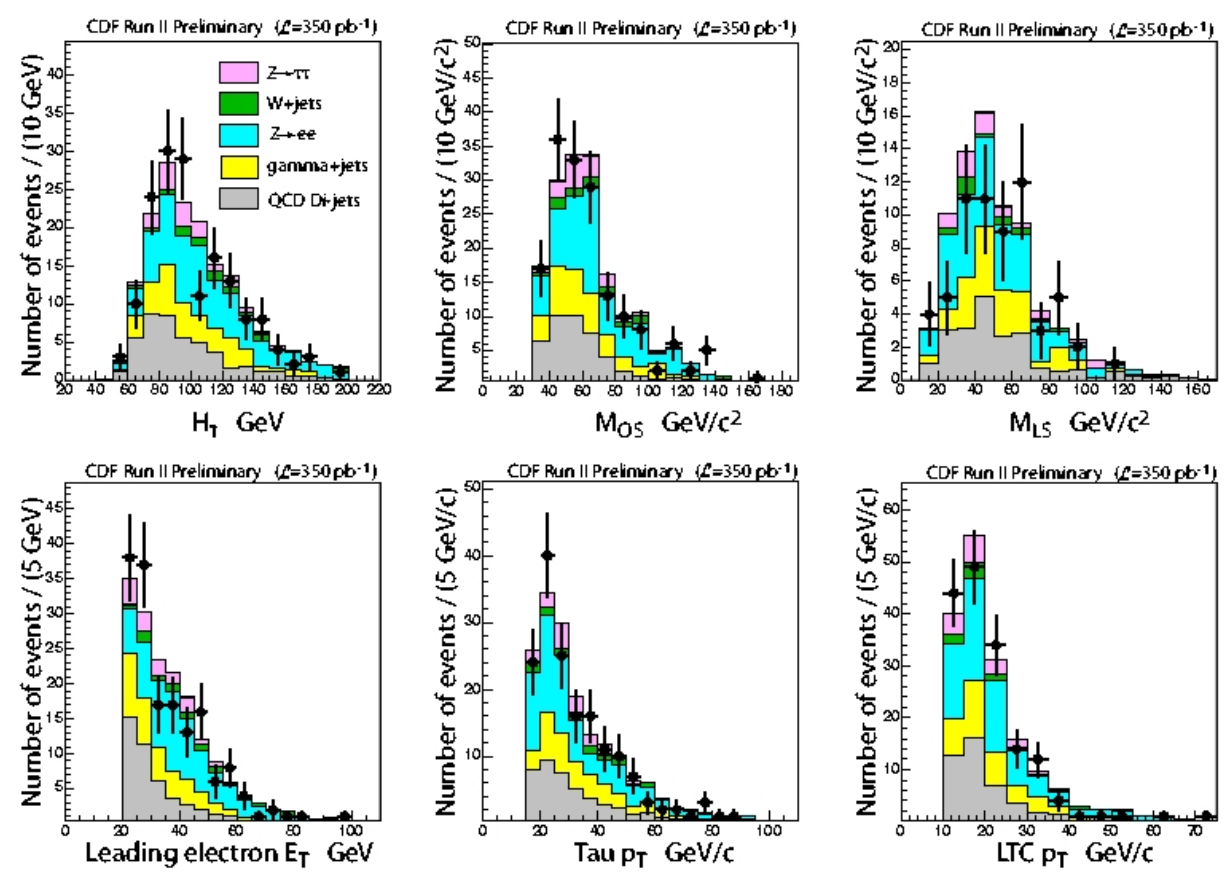

Figure 5.2: Predictions and observations for various kinematical distributions in a $\gamma+$ jets-enriched region.

the tau and LTC's track multiplicities, as shown in Figure 5.3, but depends slightly on the track isolations of the tau and LTC, as shown in Figure 5.4. This makes sense, since the total number of charged particles associated with a jet should be positively correlated with the jet's energy. If we do not correct for this relationship, an overestimation of backgrounds will result. We apply a simple correction: we fit the $H_{T}$ v TIso curve (Fig. 5.4) with a first degree polynomial, and adjust the high-TIso events by subtracting from the $H_{T}$ an amount according to the sum of the track isolations of the tau and LTC, and the slope of our linear fit. The resulting "scaled" $H_{T}$ distribution is plotted along with the "raw" $H_{T}$ for QCD and $\gamma+$ jets backgrounds in Figure 5.5. We use the difference in the fractions of the two distributions that have $H_{T}>190$ as the systematic uncertainty associated with this procedure. Of 510 events in a combined $\mathrm{QCD} / \gamma+$ jets sample, seven events pass in the raw $H_{T}$ distribution compared to four in the scaled $H_{T}$. We scale the four 


\begin{tabular}{|l|l|l|}
\hline Cuts & Loose Selections & Tight Selections \\
\hline \hline Tight ele, tau, LTC & yes & yes \\
TIso $_{\text {ele }}$ & $<2 \mathrm{GeV} / c^{2}$ & $<2 \mathrm{GeV} / c^{2}$ \\
TIso $_{\tau}$ & $<10 \mathrm{GeV} / c$ & $<2 \mathrm{GeV} / c$ \\
$N_{\tau}^{\text {Tracks }}=$ & $\leq 4$ & 1 or 3 \\
TIso LTC $_{\text {LTC }}$ & $<10 \mathrm{GeV} / c$ & $<2 \mathrm{GeV} / c$ \\
$N_{\text {LTC }}^{\text {racks }}=$ & $\leq 4$ & 1 or 3 \\
\hline
\end{tabular}

Table 5.4: Loose and tight lepton selections.

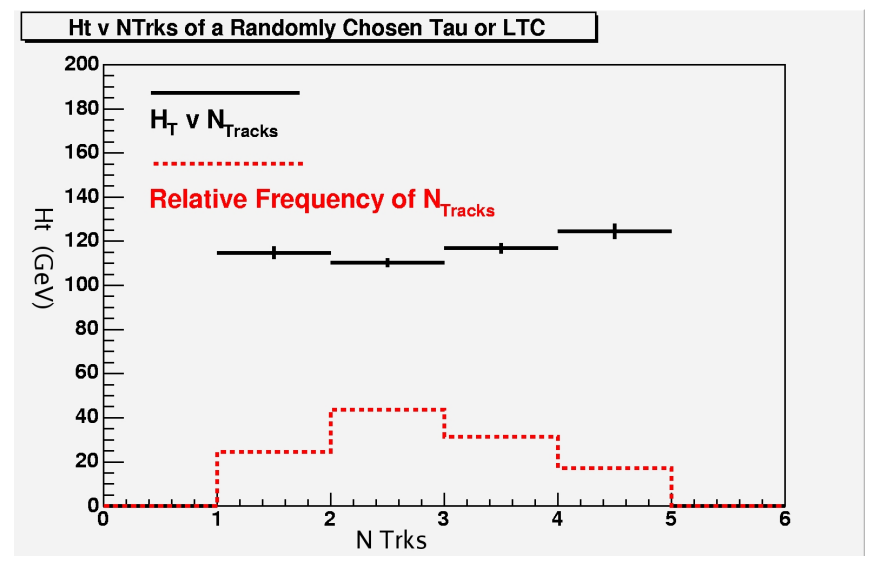

Figure 5.3: The $H_{T}$ distribution(solid black) for QCD events vs the number of tracks in the tau signal-cone for a randomly chosen tau or LTC in the event. The track multiplicity is also plotted (dashed red). The bins with the lowest and highest $H_{T}$ values are differ only by about $10 \mathrm{GeV}$.

events by the ratio $\boldsymbol{f}_{\text {Selection }} \equiv \frac{\boldsymbol{N}_{\text {Tight }}}{\boldsymbol{N}_{\text {Loose }}}$, and obtain $0.001 \pm 0.001$ (stat) \pm 0.0008 (syst) events. This procedure has essentially traded a $75 \%$ relative systematic error for a much larger statistical error that would result from using tight selections. An identical procedure is used for the $W+$ jets MC prediction.

\subsubsection{W+jets Background and Control Region}

We use a PYTHIA MC sample to estimate the $W+$ jets background and check the MC predictions in a control region enriched with $W \rightarrow e \nu_{e}+2\left(j e t \rightarrow \tau_{h}\right)$ events. We create this control region by starting with "loose lepton selections" as given by Table 5.4, and removing Drell-Yan events by requiring that there is only one tight 


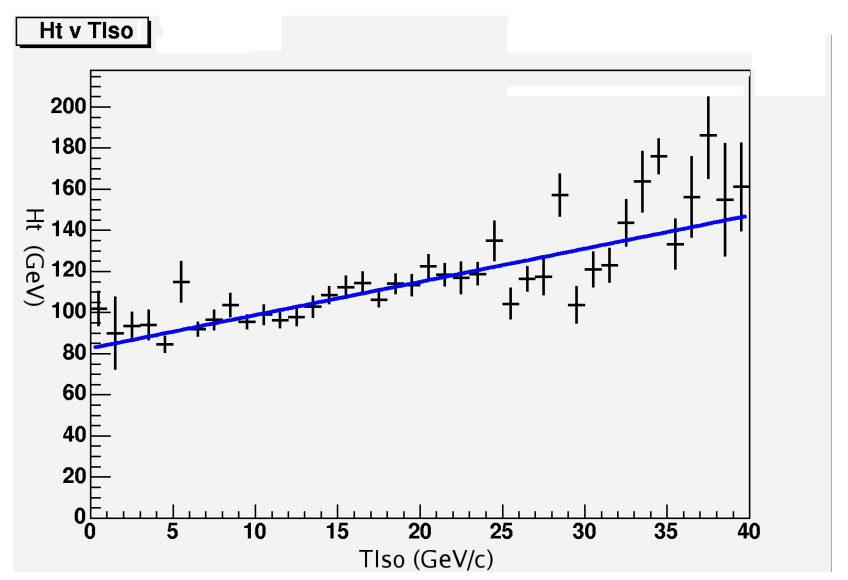

Figure 5.4: The $H_{T}$ distribution for QCD events collected with loose selections, vs the sum of the track isolations of the tau and LTC.

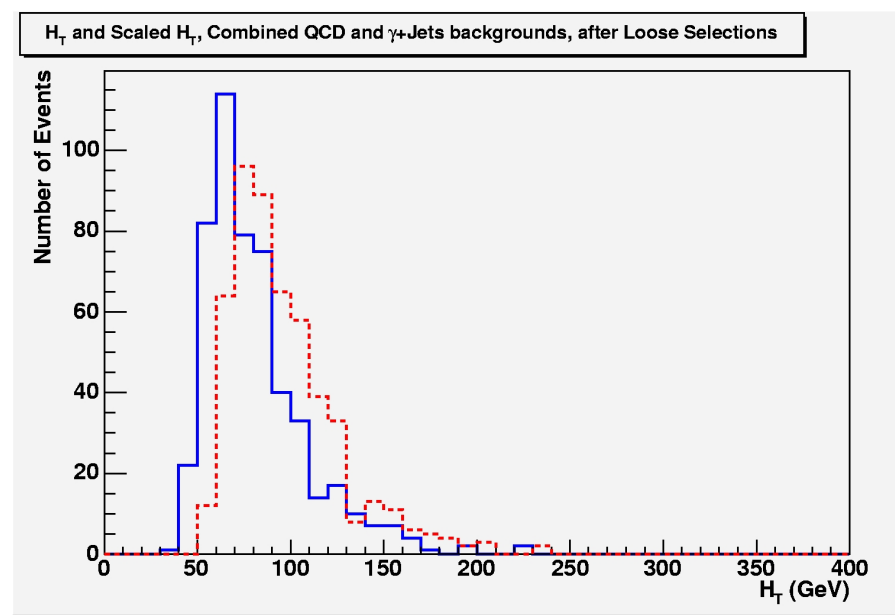

Figure 5.5: The $H_{T}$ distribution for QCD events collected with loose selections is plotted in dashed red, and the corrected $H_{T}$ is overlaid in solid blue.

isolated electron per event. To suppress QCD and $\gamma+$ jets backgrounds, we also require that events have at least $30 \mathrm{GeV}$ of $\mathbb{H}_{T}$. In this control region we see that the Monte Carlo underestimates this background. This is not surprising, as the PYTHIA implementation of jet production through ISR is not as reliable as matrixelement calculators such as ALPGEN. In particular, the $N_{\text {jets }} \geq 2$ cross section is underestimated. Because the $W+$ jets is an insignificant background ${ }^{2}$ and the

\footnotetext{
${ }^{2}$ Even when we loosen the track isolation and multiplicity requirements to get more statistics, zero events pass our analysis selections.
} 

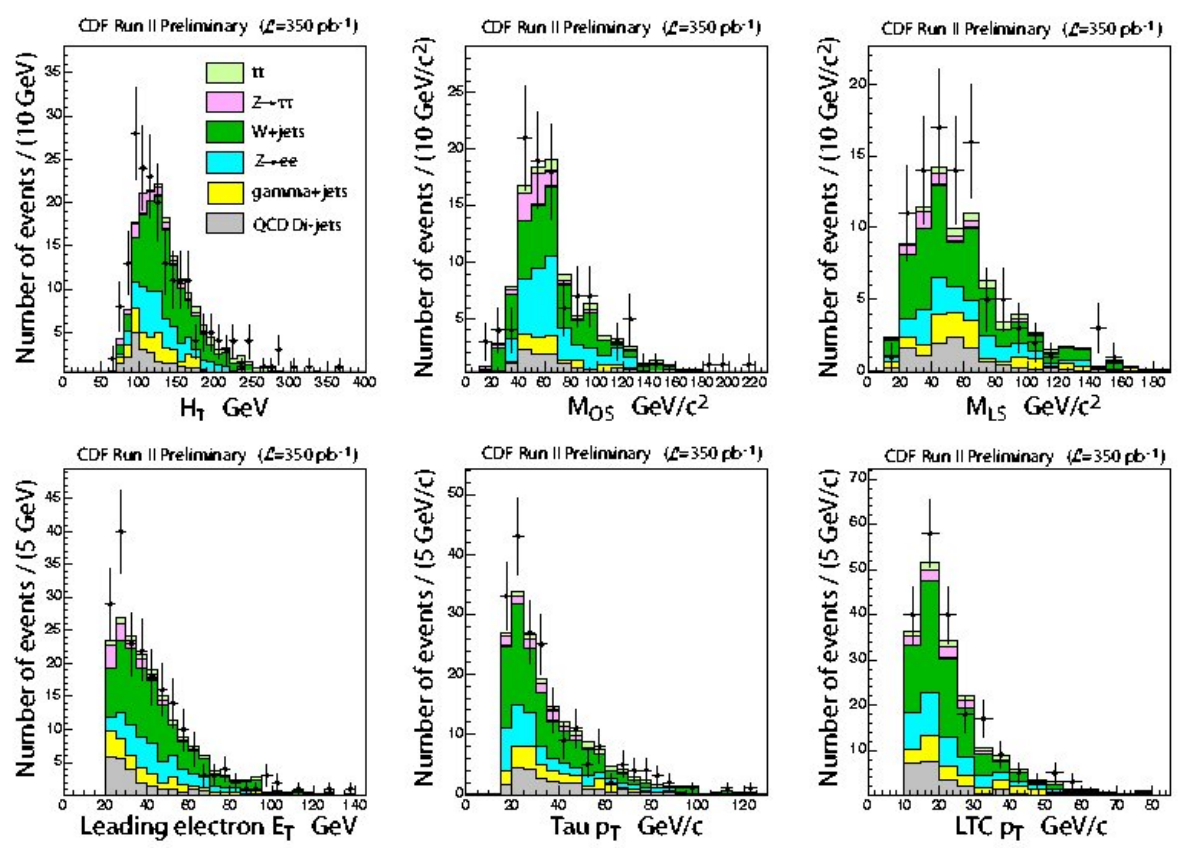

Figure 5.6: Predictions and observations for various kinematical distributions in a $W+$ jets-enriched region.

underestimation is not gross, we compensate by re-weighting the MC events. We compare the electron $+\mathscr{H}_{T}$ mass distribution between data and $\mathrm{MC}$, and calculate the $\chi^{2}$ per number of bins as we vary the overall $W+$ jets normalization. The best fit results with a scale factor of 1.28 on the $W+$ jets $\mathrm{MC}$. We use this scale factor, and take the full $28 \%$ as the systematic uncertainty associated with our determination of the $W+$ jets background. After rescaling, we check various kinematical distributions in the $\mathrm{W}+$ jets-enriched region (Figure 5.6), and see that the scaled MC predictions are reliable for the high $H_{T}$ region.

\subsection{3 $\quad Z \rightarrow e e$ Background and Control Region}

The $Z \rightarrow$ ee background enters through the associated production of a jet and its misidentification as a hadronic tau or an LTC. Unlike $W+$ jets, $Z \rightarrow e e$ is a significant background, and is reduced primarily by selections on $H_{T}$ and $M\left(e^{+} e^{-}\right)$. It is important for the (PYTHiA) Monte Carlo to be in good agreement with data 


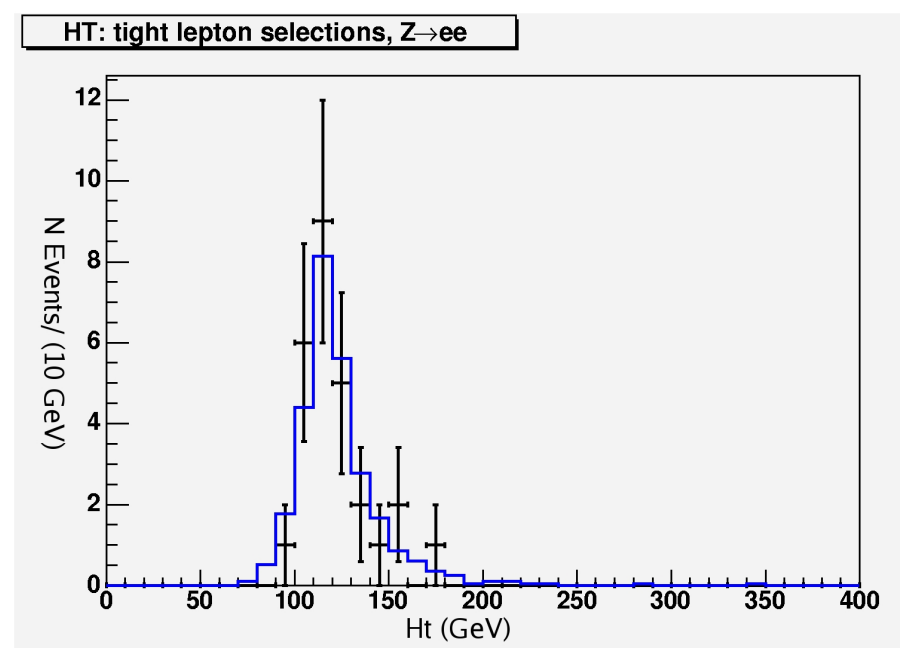

Figure 5.7: $H_{T}$ for $Z \rightarrow e e$ events in data (black markers, with statistical errors) and MC (solid blue line). These events pass all lepton identification selections, but fail Z-removal.

in the kinematics and multiplicity of jets, as well as the jet $\rightarrow \tau_{h}$ misidentification rate $^{3}$. We make two control regions to study the $Z \rightarrow e e$ background. In the first control region, we take all events that pass lepton identification, but fail the $Z$ Veto. We check the number of events and the $H_{T}$ distribution, shown in Figure 5.7, and find good agreement between MC predictions and data. In the second control sample, we drop the TIso and track multiplicity requirements on the tau and LTC to increase statistics. We check various kinematical distributions as shown by Figure 5.8. Again, we see that the agreement between data and MC is much better than in the $W+$ jets case. The ISR implementation in PYTHIA does a good job of predicting the $Z+(1)$ jet cross section and kinematics. This is not surprising, given that the dominant production mode of $Z+(1)$ jet is s-channel $Z$-production accompanied by ISR, and the ISR and FSR settings used to create our MC sample have been tuned to best match $Z$-kinematics as measured in $Z \rightarrow \mu \mu$ events at $\operatorname{CDF}[56]$.

\footnotetext{
${ }^{3}$ Discrepancies are acceptable if they are well-understood and quantified.
} 

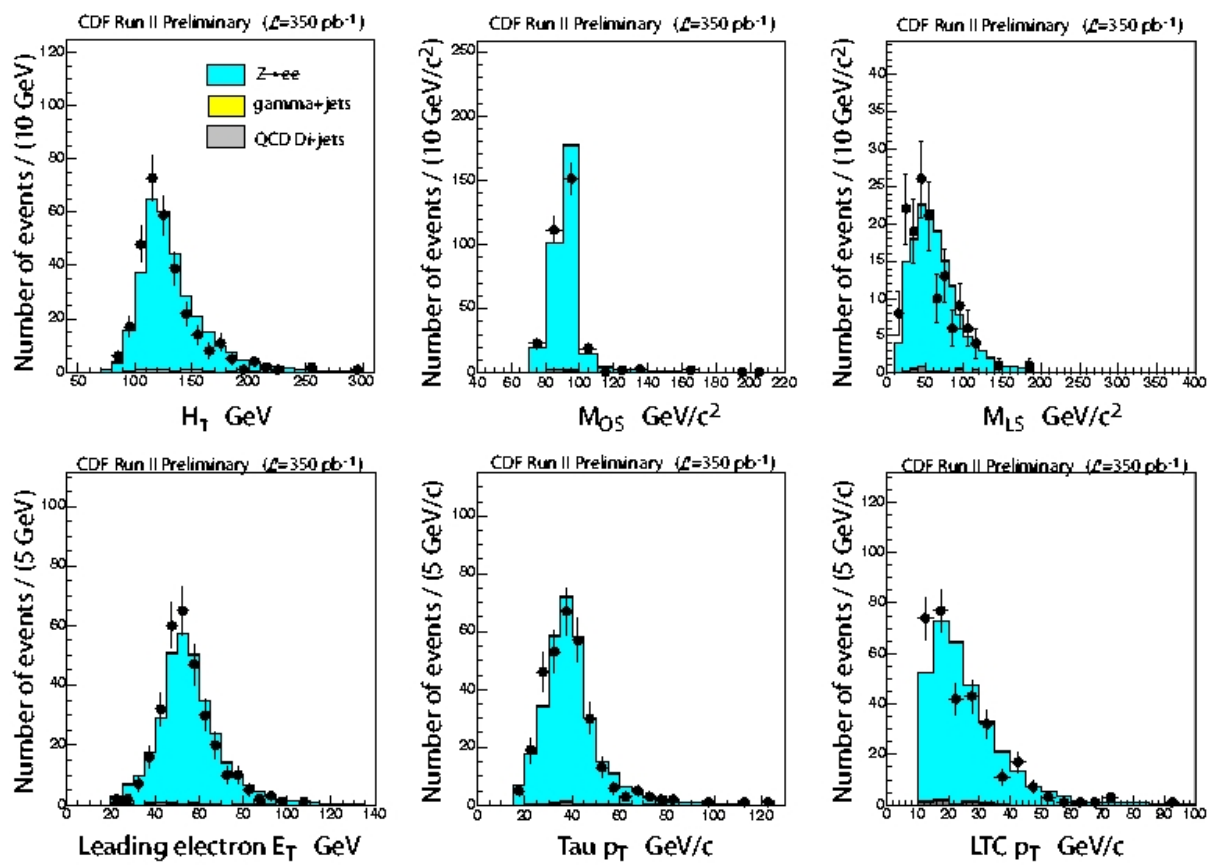

Figure 5.8: Predictions and observations for various kinematical distributions in a $Z \rightarrow e e$-enriched region.

\subsubsection{Diboson and $t \bar{t}$ Backgrounds}

Unlike QCD, $W$, and $Z$ processes, these backgrounds have such small cross sections that it is impossible to create dedicated control regions to validate MC predictions. We note that the diboson events do not rely on the associated production and subsequent misidentification of extra jets to reach our signal region. This gives us confidence that the MC predictions should be reliable. The $t \bar{t}$ mainly enters the signal region by way of the $W$ s decaying leptonically and one of the two $b$ jets being misidentified as a tau or LTC. Since $b$ s are generated as part of the "hard subprocess", their kinematics should be well-simulated compared to those of high- $p_{T}$ jets resulting from ISR. 

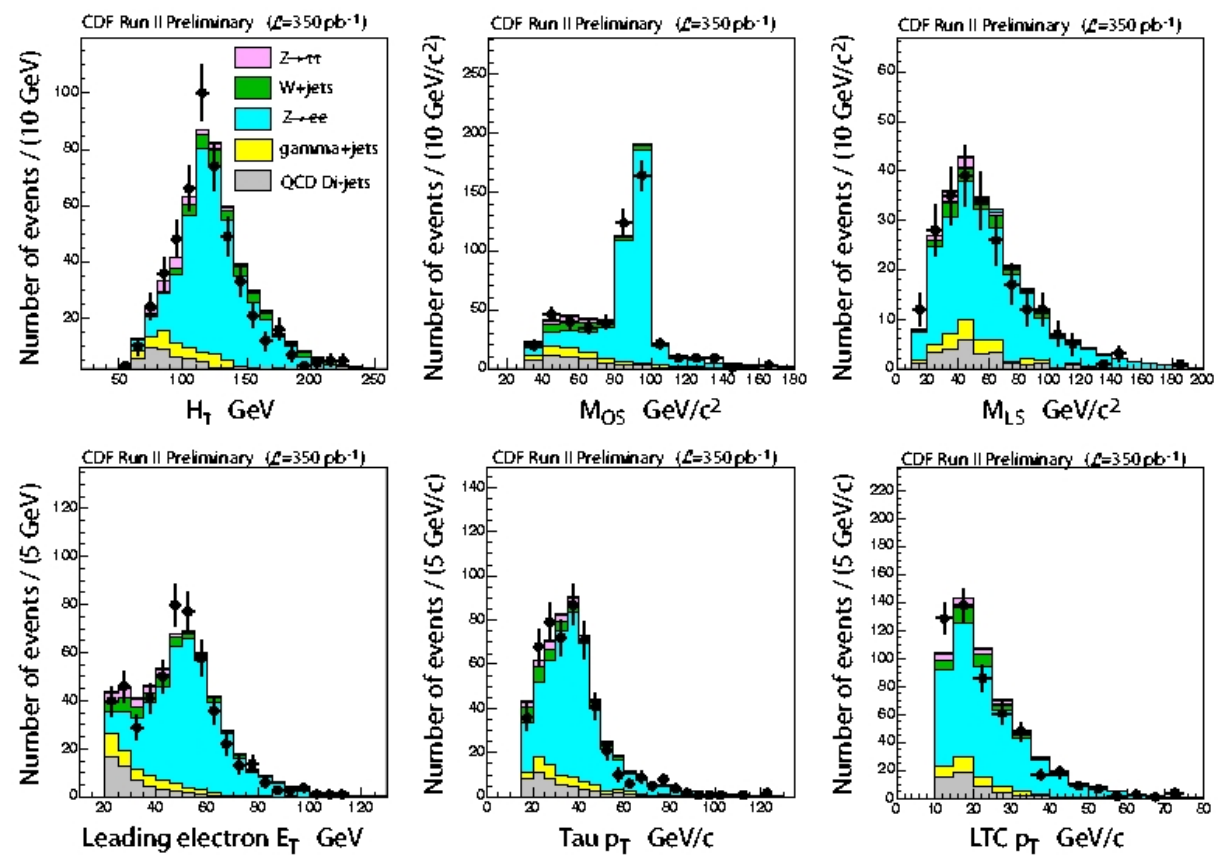

Figure 5.9: Predictions and observations for various kinematical distributions after passing loose lepton ID. (Isolated ele, unisolated Tau, LTC.) Note, since we have unblinded the analysis, we no longer restrict the $H_{T}$ plot to the region below 190 $\mathrm{GeV}$.

\subsubsection{Additional Control Regions}

\section{Loose Selections}

We use this large data sample to check for gross overall agreement. This control region results from relaxing the track isolation and track multiplicity cuts on the tau and LTC ("loose selections"). We look only up to $H_{T}<190 \mathrm{GeV}$ to stay blind to the signal, but hope to catch any aberrations in the individual components of $H_{T}$. We check the $p_{T}$ distributions of the three leptons, as well as $H_{T}$. We also check the highest invariant mass of oppositely charged leptons, and the invariant mass made by leptons of the same charge. These distributions are plotted in Figure 5.9. 

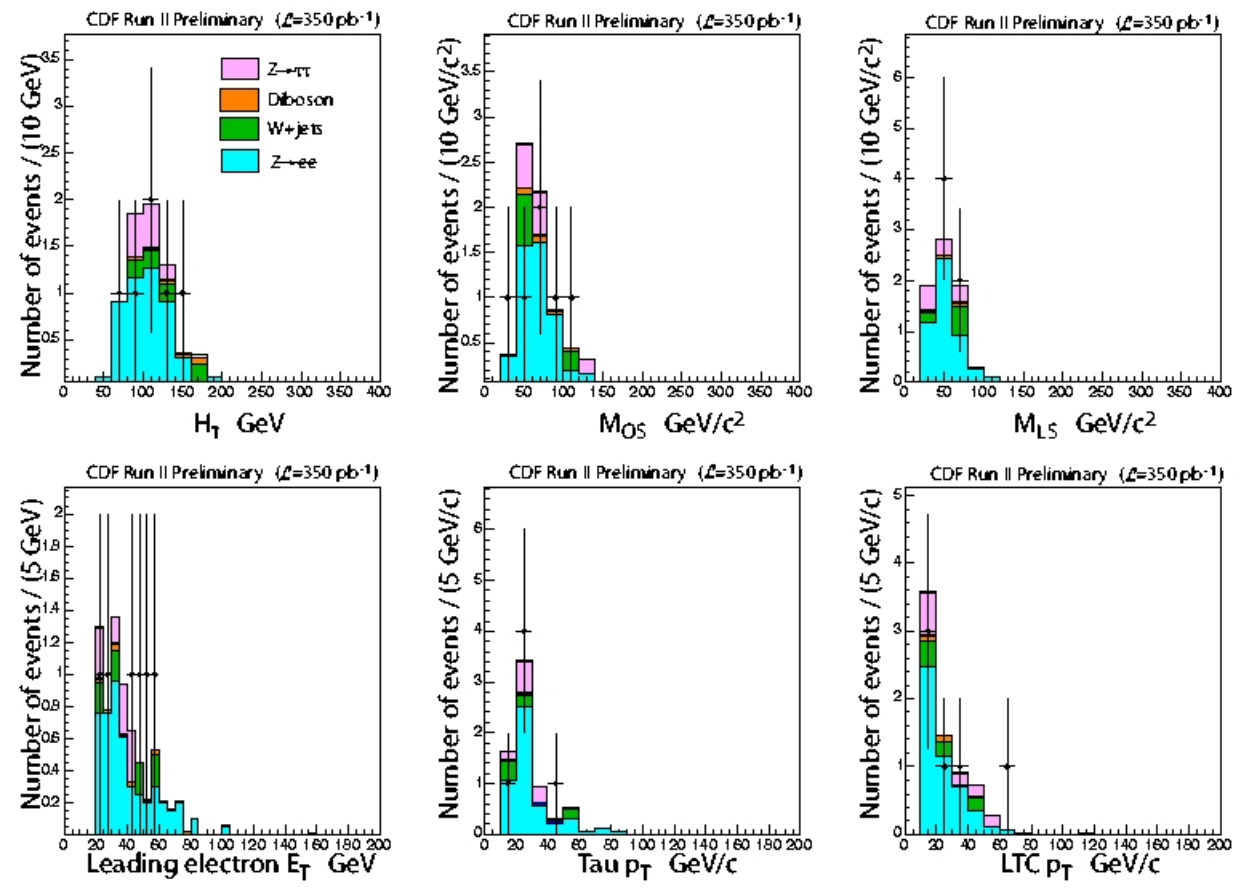

Figure 5.10: Predictions and observations for various kinematical quantities for events passing all cuts except $H_{T}$.

\subsubsection{Low $H_{T}$}

We make all lepton identification and event-level selections, except we look only in the region $H_{T}<150 \mathrm{GeV}$. We compare the same distributions as before, shown in Figure 5.10. Although there are only a few events in this control region, it is more likely that any unexpected or underestimated background will be evident here than in the signal region. This is true even for Diboson and $t \bar{t}$ backgrounds.

\subsubsection{Control Regions for the 4-Track Region}

MC studies show that the $Z Z$ process is the only appreciable background in the 4-track signal region, although before event-level cuts, we do expect 0.1 events from $Z+2$ jets. It is difficult to make control regions for the 4-track region for these reasons: 
- The number of events expected in this region is very small.

- Any event in this region will be more consistent with signal than backgrounds, unless there is an obvious $Z$ or $Z Z$ resonance.

- Even if we were to reverse the TIso on a lepton, the number of events expected from signal are still greater than the number of events from SM backgrounds.

The observation of any event with four isolated leptons would be interesting, and any such event should be investigated in detail. Our strategy is to prepare for such investigations in the event of an observation. To this end, our analysis code can print out detailed information regarding every lepton-candidate and jet in the event, and six different measurements of the $\mathbb{F}_{T}$ that result from various levels of corrections. Furthermore, all relevant kinematical quantities, such as the invariant masses made by momenta of the particles and the $\mathbb{F}_{T} \mathrm{~s}$ are printed out. Interesting events can also be filtered out and studied in detail with the aid of a visual event display. As a side-note, we do not observe any 4-lepton events in either the $e \tau$ or $\mu \tau$ searches, but we observe one event in the $\mu \tau$ search that passes all 3 -track selections except for the like-sign mass cut. We use some of the above tools to characterize this event in the appendix.

\subsection{Backgrounds and Control Regions for the $\mu \tau$ Search}

\section{Estimation of $\mu \tau$ Backgrounds}

Our background estimations for the $\mu \tau$ search are summarized in Table 5.5. The background types and levels are analogous to the er search, with the exception that the $\gamma+$ jets background is replaced with an equally negligible cosmic ray muon background.

Cosmic ray muons can enter as backgrounds if they can overlap with an event that provides a third isolated lepton. CDF's powerful cosmic rejection reduces the cosmic contamination to $\sim 1 \%$ of the inclusive $Z \rightarrow \mu \mu$ background level[23]. Our 
cosmic backgrounds are even lower as the cosmic ray and jet would need to overlap in the $z$-coordinate. One can conservatively take the cosmic background level as $1 \%$ of the $Z(\rightarrow \mu \mu+$ jets $)$ level, or 0.0005 events $^{4}$.

The QCD background is estimated by the same track isolation extrapolation

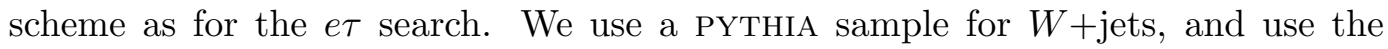
same scale factor and systematical uncertainty as used for the $e \tau$ search, because the jet multiplicities and kinematics are independent of the leptonic decay mode $(e$ or $\mu$ ) of the $W$ s. Also, the same method as described in section 5.3.1 is used to increase the statistical precision of these background predictions. We use PYTHIA samples to determine the $Z+$ jets backgrounds. The jet $p_{T}$ and event $H_{T}$ validations of the $e \tau$ search are applicable because the same generation/simulation scheme has been used. The only relevant new aspects are specific to muons, and are checked in dedicated control regions as described below. The same Top and Diboson MC samples are used here as in the $e \tau$ search.

The $\mu \tau$ search has a slightly different set of control regions than the $e \tau$ search. The QCD and $W+$ jets backgrounds are expected to be as insignificant as they were in the $e \tau$ search, and therefore we do not make dedicated control regions for these backgrounds, but check them in more inclusive control regions. On the other hand, we wish to understand and minimize the risk of spuriously high $H_{T}$ which may result from gross mismeasurement of muons $p_{T}$ s. These kinds of events are extremely rare, therefore we must make control samples with a very large acceptance. The large acceptance also reduces associated statistical errors and enables us to test acceptance and trigger simulation scale factors for muons by comparing the CMUP and CMX acceptances in data and MC.

\subsubsection{Dilepton Control Region}

This control region consists of one tight isolated muon and one tight isolated tau. The only event-level cuts are cosmic removal and the curvature error cut on the

\footnotetext{
${ }^{4}$ Instead, we throw caution to the (cosmic) wind and treat this background as non-existent.
} 


\begin{tabular}{|c|c|c|c|}
\hline \hline \multicolumn{4}{|c|}{$\boldsymbol{\mu \tau}$ Background Predictions } \\
\hline \hline Process & Method & NEv in 3-Lepton & NEv in 4-Lepton \\
\hline$Z+$ jets & PYTHIA MC & $0.10_{-0.1}^{+0.18}$ & $0.05_{-0.0}^{+0.05}$ \\
\hline Dibosons & PYTHIA/MADGRAPH MC & $0.11^{+0.01}$ & $0.10 \pm 0.01$ \\
\hline$t \bar{t}$ & PYTHIA MC & $0.06 \pm 0.02$ & $0_{-0.0}^{+0.01}$ \\
\hline$W \rightarrow e \nu_{e}+$ jets & PYTHIA MC & $0.0_{-0.0}^{+0.19}$ & $0.0_{-0.0}^{+0.01}$ \\
\hline $\mathrm{QCD} /$ Cosmics & Data & $0.001 \pm 0.001$ & $0 !$ \\
\hline \hline Total & & $\mathbf{0 . 2 7}_{-\mathbf{0 . 1 1}}^{+0.26}$ & $\mathbf{0 . 1 6} \pm \mathbf{0 . 0 5}$ \\
\hline
\end{tabular}

Table 5.5: Background estimations for the $\mu \tau$ search. Both statistical and systematic errors are included in quadrature.

leading muon's track. This control region contains the most events, and various comparisons are useful for checking the MC normalizations for $Z$ and $W$ events, trigger simulation, and various scale factors used for lepton reconstruction and ID $^{5}$. The plots in Figure 5.11 demonstrate several checks.

- The relatively large QCD content in this region enables validation of our QCD estimation.

- The $W+1$ jet background is well predicted as shown by the $M\left(\mu, \mathbb{F}_{T}\right)$ distribution.

- The muon ID and trigger scale factors look to be correct, as shown by the CMUP/CMX ratio of the leading muons.

- The $p_{T}$ spectrum of the leading muon does not show any excess of events in the very high $p_{T}$ region, leading us to believe that the risk of severely mismeasured muon $p_{T}$ is less than $\mathcal{O}(1 / 500)$.

The last two points were the main checks that we wanted to make with this control region.

\footnotetext{
${ }^{5}$ The only scale factors that appreciably differ from unity are the muon stub-finding scale factors, which we implement in a fashion identical to muon trigger-efficiency scale factors.
} 

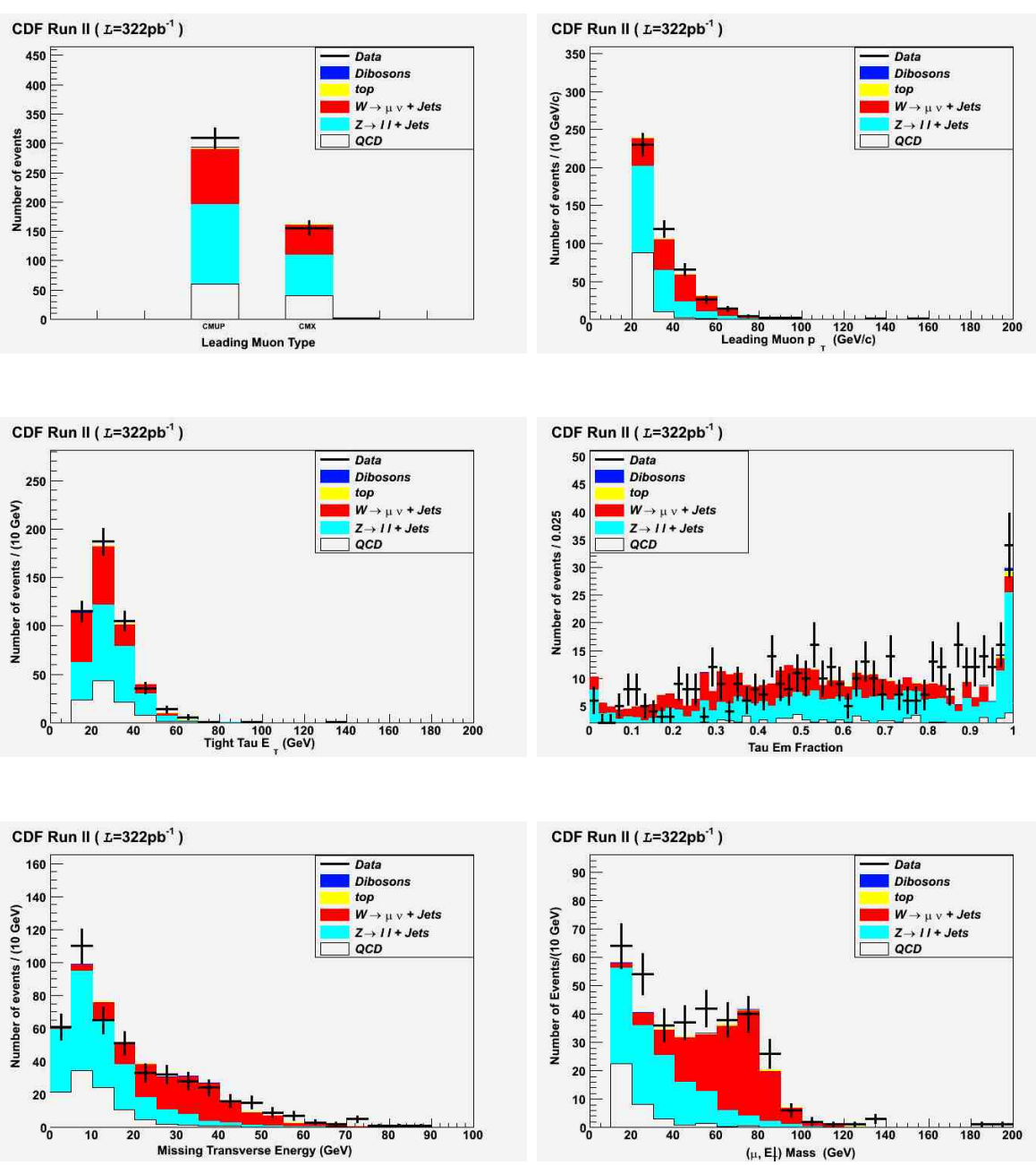

Figure 5.11: Plots for the Dilepton Control region. The first column of plots from top to bottom are: the type of the leading muon (CMUP/CMX), the $E_{T}$ of the tau, and the $\mathbb{F}_{T}$. The second column has from top to bottom: the muon $p_{T}$, the EM fraction of the tau, and the invariant mass made by the muon and $\mathbb{F}_{T}$.

\subsubsection{Loose Selections}

This control region is similar to the corresponding one in the $e \tau$ search. We require three tight leptons, but relax the track isolation and track multiplicity requirements on the tau and ITS. To suppress the signal in this region, we look only at $H_{T}<$ $190 \mathrm{GeV}$. In Figure 5.12 we check the leading muon type (CMX and CMUP) and also the $p_{T} \mathrm{~s}$ of the three leptons. In Figure 5.13 we check the various incarnations of 

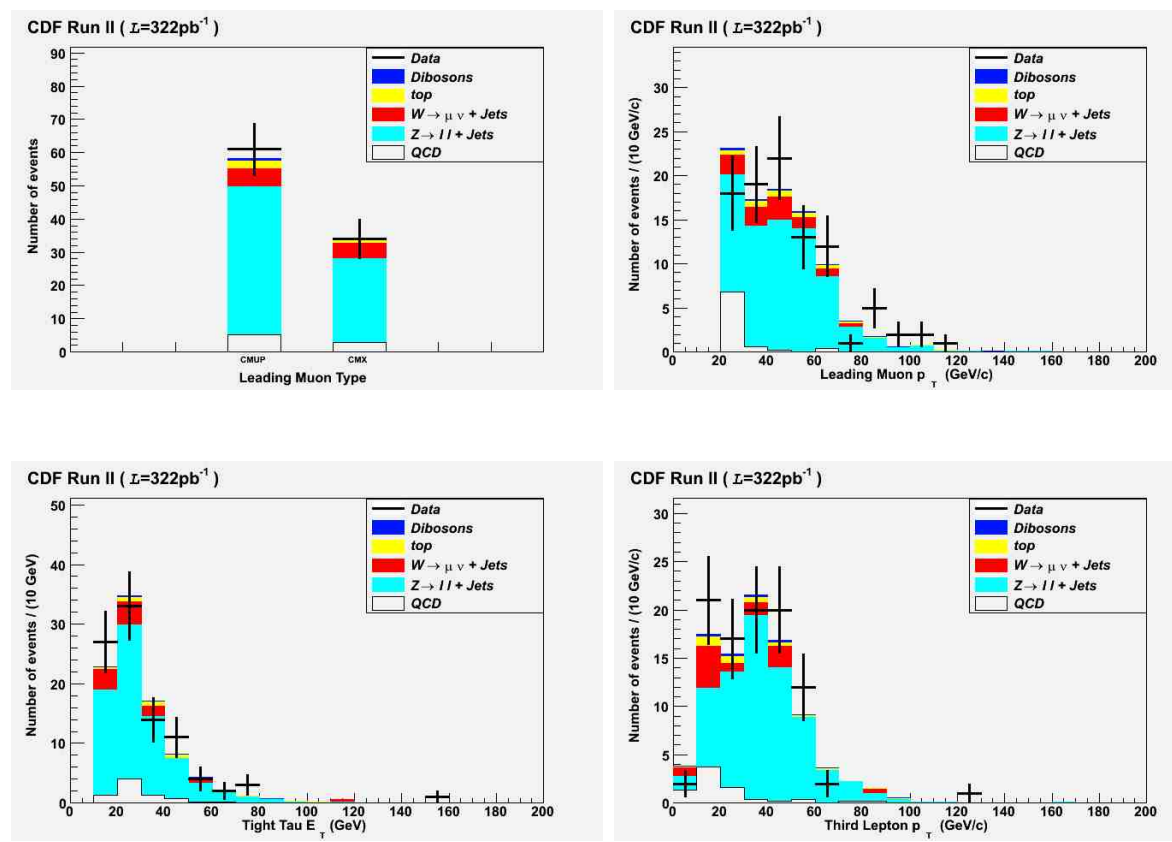

Figure 5.12: Plots from the control region with isolation applied to the leading muon only. The top-left plot shows the type of the leading muon (CMX or CMUP). The top-right plot shows the leading muon $p_{T}$. The bottom-left plot is the $E_{T}$ of the tau, while the bottom-right plot shows the $p_{T}$ of the third lepton.

the ITS, and find good agreement between data and MC predictions. We also check the corrected $\mathbb{F}_{T}$, the highest invariant mass made by the leading muon and any other oppositely charged particle in the event, and the $H_{T}$. We find good overall agreement between our predictions and data.

\subsubsection{Low $H_{T}$}

We look at events that pass all analysis selections except for $H_{T}$ and $Z$-Veto. As with the $e \tau$ analysis, there are not many events in this region, however, as most SM backgrounds prefer this region to the high $H_{T}$ region, any significant deviation from predictions regarding misidentification rates or MC normalizations are more likely to manifest here than in the signal region. The individual components of $H_{T}$ are plotted in Figure 5.14 for this control region. 

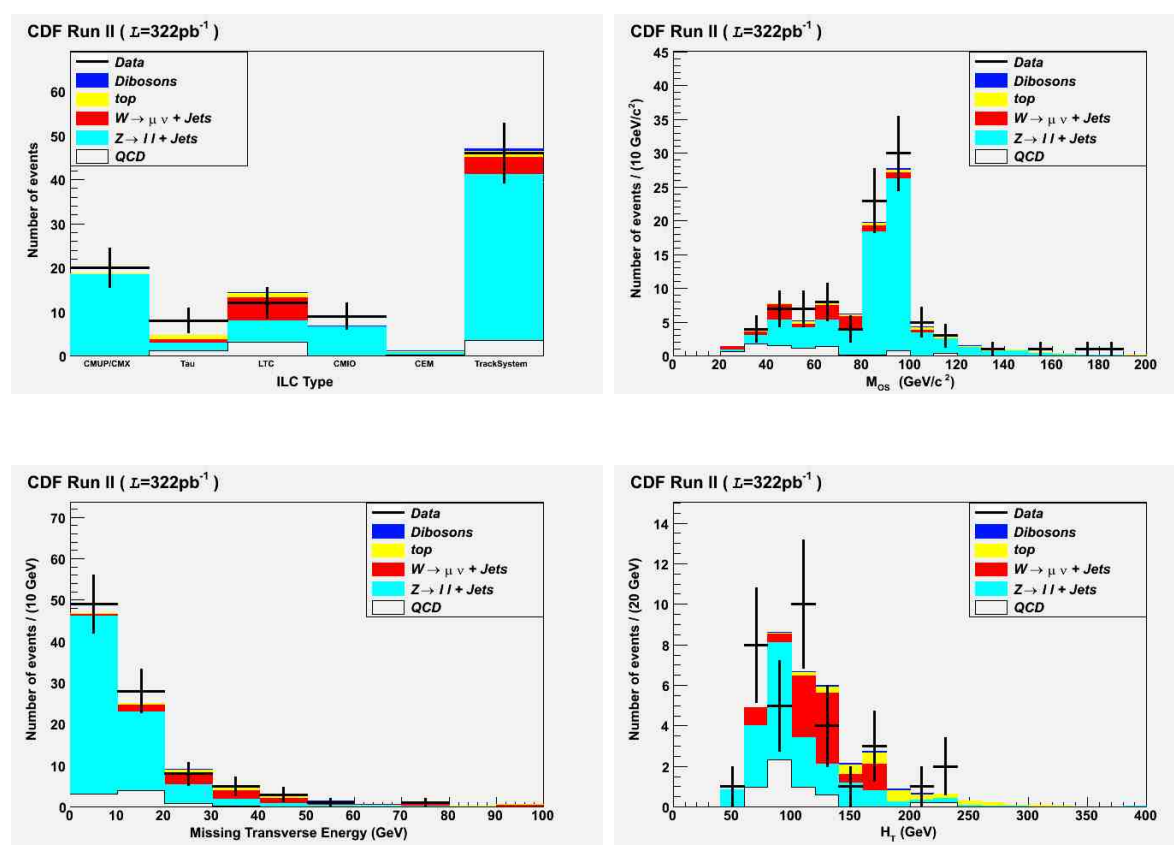

Figure 5.13: Plots from the control region with isolation applied to the leading muon only. The top-left plot shows the reconstructed objects corresponding to the third lepton - they all pass as ITSs, but about $50 \%$ of them also pass some other more stringent requirement as shown. The top-right plot shows the highest invariant mass made by opposite-sign leptons in the event. The bottom-left plot is the $\mathbb{F}_{T}$, while the bottom-right is the $H_{T}$. Note, since we have unblinded the analysis, we no longer restrict the $H_{T}$ plot to the region below $190 \mathrm{GeV}$.

\subsubsection{Reverse Tau TIso (high $H_{T}$ )}

It is important to check backgrounds at high $H_{T}$. Since all relevant SM backgrounds seem to be accounted for in the first three control regions, it is assumed that ${ }^{6}$ any excess backgrounds in the high $H_{T}$ region would most likely result from rare instrumental effects (e.g. the mismeasurement of a muon resulting in an erroneous adjustment of the $\mathbb{F}_{T}$, possibly leading to artificially high $H_{T}$, or rare cases where the EM or Hadronic calorimeter severely overestimates a particle's energy). We reverse the track isolation on the hadronic tau, thereby suppressing signal but increasing background acceptance, and, in Figure 5.15 look in the high $H_{T}$ region. It is expected that such aforementioned instrumental effects are independent of the

\footnotetext{
${ }^{6}$ Apart from new physics.
} 

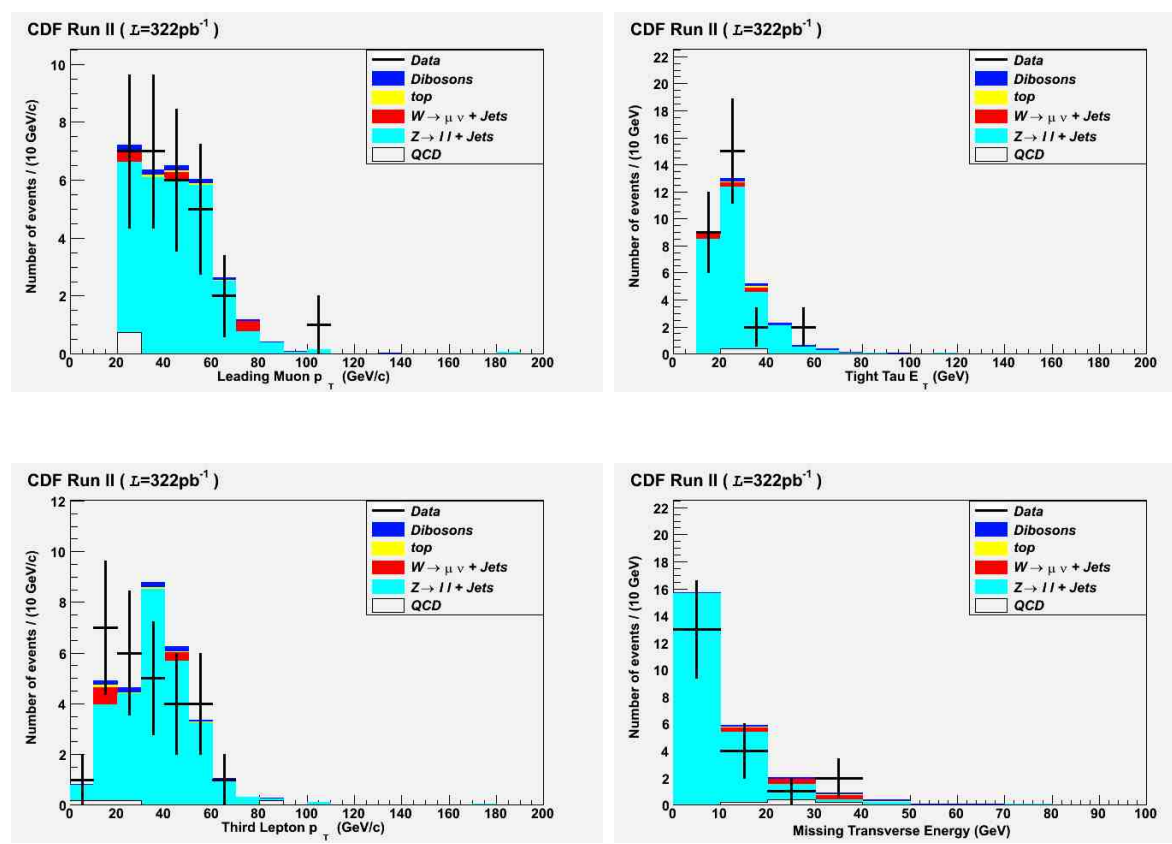

Figure 5.14: Plots from the low $H_{T}$ control region. From top-left to bottom-right we have leading muon $p_{T}$, tau $p_{T}$, ITS $p_{T}$, and $\mathscr{f}_{T}$.

$\tau_{h}$ 's track isolation, therefore we have a higher chance of observing such phenomena here than in the signal region, due to this control region's larger sample size.

\subsubsection{Mismeasured Track $p_{T}$ Study with $Z \rightarrow e e$ Data}

To check the efficacy of the $\sigma_{C u r v}$ cut on the highest- $p_{T}$ track in the event, we apply the cut to a $Z \rightarrow e e$ sample from data. We select events passing the CEM+track trigger that have two oppositely charged, well-identified electrons that make in invariant mass in the range $88 \mathrm{GeV} / c^{2}<M\left(e^{+}, e^{-}\right)<94 \mathrm{GeV} / c^{2}$. We then take the electron with the highest $p_{T}$ track and measure $c \cdot p_{T}-E_{T}$, where $c$ is the speed of light (we use units $c=1$ ). This quantity may be negative if the electron undergoes severe Bremsstrahlung, so we remove such events by requiring that $c \cdot p_{T}-E_{T}>-2 \mathrm{GeV}$. This requirement ensures that the track and calorimetry measurements are in agreement within $\sim 1 \sigma$ of the combined resolution of the tracking and calorimetry, but only for the cases where the track $p_{T}$ s lower than the $E_{T}$ in the EM calorimeter. 


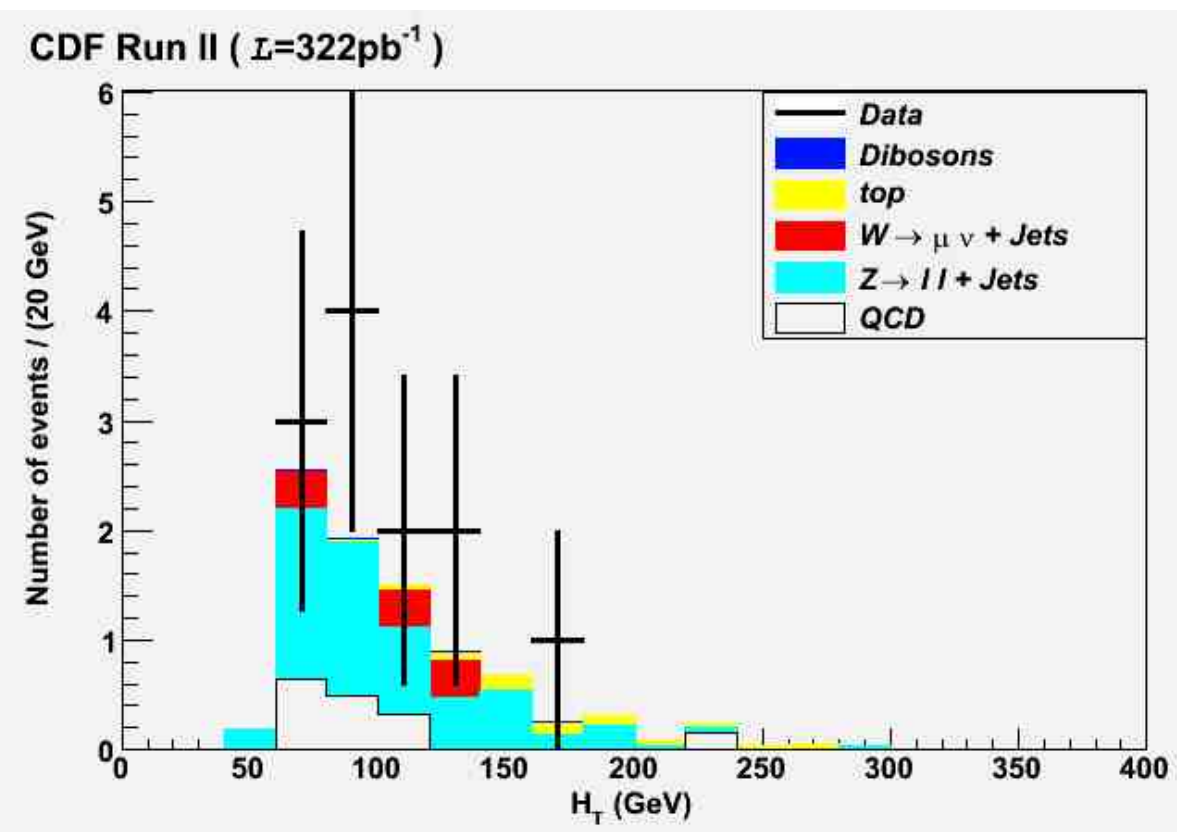

Figure 5.15: The $H_{T}$ distribution for events that pass all analysis selections except for tau TIso (and $H_{T}$ ).

We let the distribution be unrestricted on the other end of the spectrum to quantify the frequency with which the track $p_{T}$ is much more than the EM $E_{T}$. Figure 5.16 shows this distribution before and after applying the cut on the track curvature resolution. We see that the cut effectively removes events with mismeasured track $p_{T} \mathrm{~s}$, and also that the rate of severely mismeasured tracks that remain is under $1 / 1000$, which is consistent with our findings in the dilepton control region.

\subsection{Systematic Errors on Background Acceptance}

The backgrounds that are determined by MC have uncertainties associated with the knowledge of the true production cross section process. These errors, sometimes called "Next to Leading Order" (NLO) errors, are $\sim 8 \%$ relative to the predicted $\mathrm{MC}$ cross section for the top and diboson processes, and somewhat smaller for the inclusive $Z$ and $W$ processes. We require the associated jets with the $Z \mathrm{~s}$ or $W$ s, and this may increase the systematic uncertainty. For example, we take the systematic 


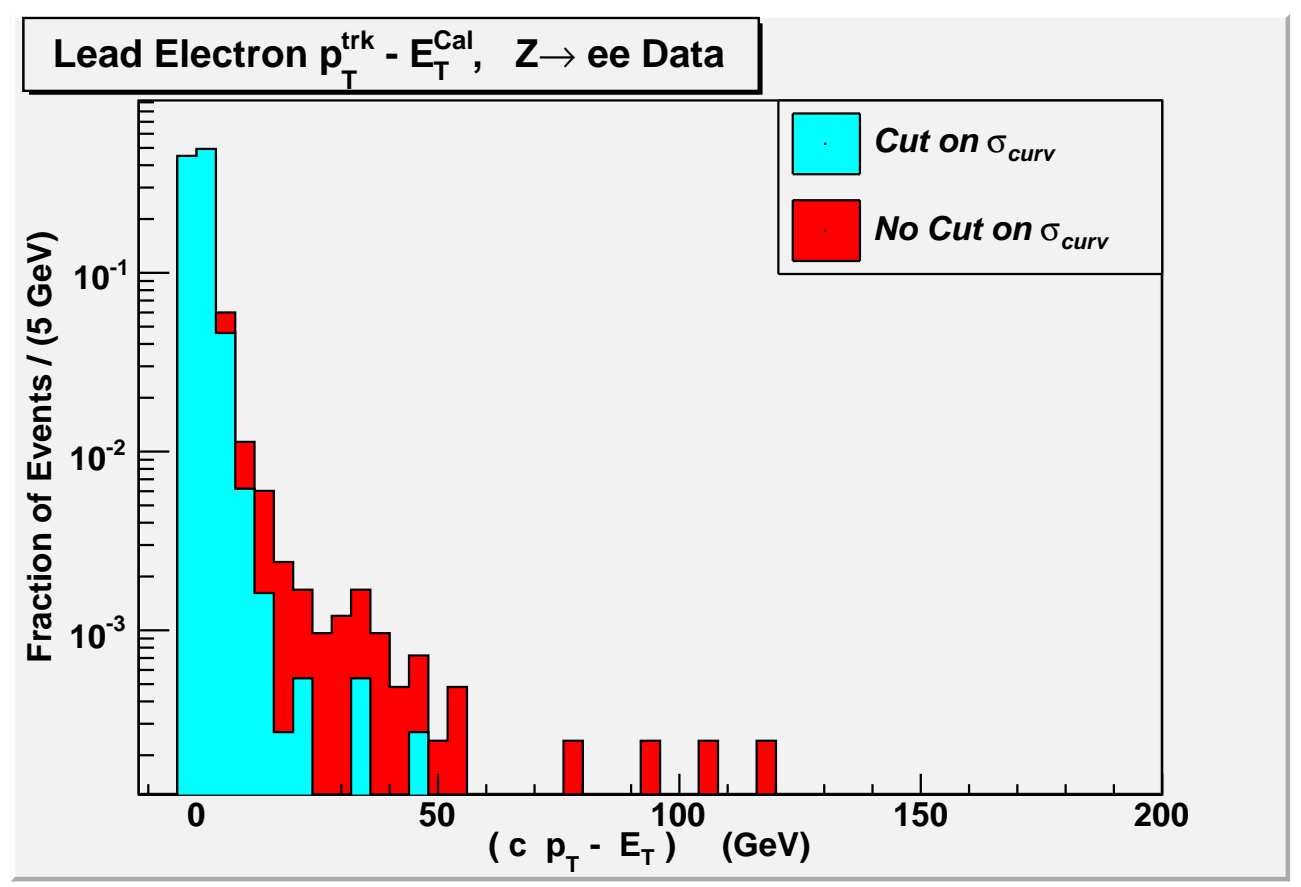

Figure 5.16: This plot shows the $c \cdot p_{T}-E_{T}$ for the higher- $p_{T}$ electron in a $Z \rightarrow$ ee sample collected from Data. Bremsstrahlung events are removed by requiring the $p_{T}-E_{T}>-2$. The red distribution is before the cut on $\sigma_{\text {curv }}$ while the cyan distribution remains after the $\sigma_{\text {curv }}$ cut. The rate of severe mismeasuremet $\left(c \cdot p_{T}-E_{T}>20 \mathrm{GeV}\right)$ is reduced from 0.004 to 0.0005 . (Note that the statistics are double what is shown here because only the higher- $p_{T}$ electron makes it to this plot.)

uncertainty of the $W+\geq 2$ jets cross section to be $28 \%$. We see good agreement in the $Z+(1)$ jet predictions, so we assign an $\sim 5 \%$ uncertainty on overall $Z+(1)$ jet cross section based on the statistical uncertainty on the number of observed events in the $Z+(1)$ jet-enriched control region. We assign a 0.1 event systematic uncertainty to account for potentially imperfect MC simulation of the efficacy of the $\sigma_{C u r v}$ cut. This is very conservative, given that we've measured the absolute rate for such backgrounds in data to be less than 1 per mil, and, given that only $~ 30$ events pass our lepton selections, this rate corresponds to 0.03 potential events. Other sources of systematic uncertainty, such as determination of luminosity, are discussed in the next section. 


\section{Chapter 6}

\section{Signal Acceptance}

\subsection{Expected Signal}

The predicted and observed numbers of events for signal and backgrounds at various stages of the $e \tau$ and $\mu \tau$ analyses are summarized in Tables 6.1 and 6.2 respectively. Figure 6.1 shows the combined acceptance of the three- and four-lepton channels vs. $H^{++}$mass. The $e \tau$ and $\mu \tau$ searches have similar acceptances, $(11.5 \%$ vs $11.9 \%$ for $\left.\mathrm{M}\left(H^{ \pm \pm}\right)=110 \mathrm{GeV} / c^{2}\right)$. The statistical errors on the acceptances are small due to sufficiently large MC samples. The systematical uncertainties on acceptance are discussed in the following section.

\begin{tabular}{|c|c|c|c|c|c|c|c|}
\hline \multicolumn{8}{|c|}{ et Signal/Background Acceptance } \\
\hline & $110 \mathrm{GeV}$ & $\overline{\text { Bkgnds }}$ & Data & $\overline{\text { Dibosons }}$ & $\overline{Z+\text { jets }}$ & top & $\overline{W+\text { jets }}$ \\
\hline $\mathrm{NEv}$ & 31.3 & $>1.3 \mathrm{e} .6$ & $\mathrm{~N} / \mathrm{A}$ & 126 & $340 \mathrm{k}$ & $2.3 \mathrm{k}$ & $970 \mathrm{k}$ \\
\hline Lepton ID & $4.55 \pm 0.09$ & $36.6 \pm 1.2$ & 34 & $0.81 \pm 0.03$ & $34.55 \pm 1.2$ & $0.09 \pm 0.03$ & $1.17 \pm 0.28$ \\
\hline \multicolumn{8}{|c|}{ 3-Lepton Events } \\
\hline 3-Lepton & $2.94 \pm 0.09$ & $36.43 \pm 1.2$ & 34 & $0.73 \pm 0.03$ & $34.45 \pm 1.2$ & $0.08 \pm 0.02$ & $1.17 \pm 0.28$ \\
\hline$M_{L S} / M_{O S}$ & $2.89 \pm 0.09$ & $32.1 \pm 1.1$ & 29 & $0.67 \pm 0.03$ & $30.4+.-1.1$ & $0.06 \pm 0.02$ & $0.97 \pm 0.26$ \\
\hline Z-Veto & $2.40 \pm 0.08$ & $7.01^{ \pm} 0.5$ & 8 & $0.29 \pm .02$ & $5.90 \pm 0.4$ & $0.04 \pm 0.01$ & $0.78 \pm 0.23$ \\
\hline$H_{T}>190$ & $1.97 \pm 0.07$ & $0.23_{-0.16}^{+0.26}$ & 0 & $0.06 \pm .01$ & $0.16_{-0.16}^{+0.16}$ & $0.01 \pm 0.01$ & $0.0_{-0.0}^{+0.2}$ \\
\hline \multicolumn{8}{|c|}{ 4-Lepton Events } \\
\hline 4-Lepton & $1.61 \pm .06$ & $0.18 \pm 0.05$ & 0 & $.08 \pm 0.01$ & $0.1 \pm 0.05$ & $0_{-0.0}^{+0.01}$ & $0_{0.0}^{+0.01}$ \\
\hline Z-Veto & $1.61 \pm .06$ & $0.05_{-0.05}^{+0.07}$ & 0 & $.05 \pm .01$ & $0_{-0.0}^{+0.05}$ & $0_{-0.0}^{+0.01}$ & $0_{0.0}^{+0.01}$ \\
\hline Emfr Cut & $1.6 \pm .06$ & $0.04_{-0.04}^{+0.07}$ & 0 & $.04 \pm .01$ & $0_{-0.0}^{+0.05}$ & $0_{-0.0}^{+0.01}$ & $0_{0.0}^{+0.01}$ \\
\hline
\end{tabular}

Table 6.1: Signal and background expectations, and observed events in data, for the et search. 


\begin{tabular}{|c|c|c|c|c|c|c|c|}
\hline \hline \multicolumn{10}{|c}{$\boldsymbol{\tau}$ Signal/Background Acceptance } \\
\hline \hline & $110 \mathrm{GeV}$ & Bkgnds & Data & Dibosons & $Z+$ jets & top & $W+$ jets \\
\hline NEv & 28.8 & $>1.2 \mathrm{e} .6$ & N/A & 115.6 & $320 \mathrm{k}$ & $2.2 \mathrm{k}$ & $892 \mathrm{k}$ \\
\hline Lepton ID & 4.71 & $30.2 \pm 1.2$ & 28 & $1.08 \pm 0.03$ & $27.8 \pm 1.1$ & $0.29 \pm 0.05$ & $1.02 \pm 0.33$ \\
\hline \hline \multicolumn{8}{|c|}{ 3-Lepton Events } \\
\hline 3-Lepton & $3.06 \pm 0.05$ & $30.0 \pm 1.2$ & 28 & $1.01 \pm 0.03$ & $27.7 \pm 1.1$ & $0.29 \pm 0.05$ & $1.02 \pm 0.33$ \\
\hline Not Cosmic & $3.05 \pm 0.04$ & $29.9 \pm 1.2$ & 28 & $1.01 \pm 0.03$ & $27.6 \pm 1.1$ & $0.29 \pm 0.05$ & $1.02 \pm 0.33$ \\
\hline$M_{L S} / M O S$ & $2.99 \pm 0.04$ & $25.3 \pm 1.2$ & 20 & $0.78 \pm 0.02$ & $23.2 \pm 1.1$ & $0.28 \pm 0.05$ & $1.02 \pm 0.33$ \\
\hline Z-Veto & $2.37 \pm 0.04$ & $6.56 \pm 0.7$ & 7 & $0.39 \pm 0.02$ & $4.9 \pm 0.61$ & $0.25 \pm 0.05$ & $1.02 \pm 0.33$ \\
\hline$H_{T}>190$ & $1.80 \pm 0.04$ & $0.27 \pm 0.25$ & 0 & $0.11 \pm 0.01$ & $0.10_{-0.1}^{+0.16}$ & $0.06 \pm 0.02$ & $0.0_{-0.0}^{+0.19}$ \\
\hline \hline \multicolumn{8}{|c|}{ 4-Lepton Events } \\
\hline 4 -Lepton & $1.64 \pm 0.03$ & $0.27 \pm 0.05$ & 0 & $0.11 \pm 0.01$ & $0.15 \pm 0.05$ & $0.01 \pm 0.01$ & $0_{-0.0}^{+0.01}$ \\
\hline $\begin{array}{c}H_{T}>100 \\
(>150 \text { if } Z)\end{array}$ & $1.63 \pm 0.03$ & $0.16 \pm 0.05$ & 0 & $0.10 \pm .01$ & $0.05 \pm 0.05$ & $0.01 \pm .01$ & $0_{-0}^{+0.01}$ \\
\hline
\end{tabular}

Table 6.2: Signal and background expectations, and observed events in data, for the $\mu \tau$ search.

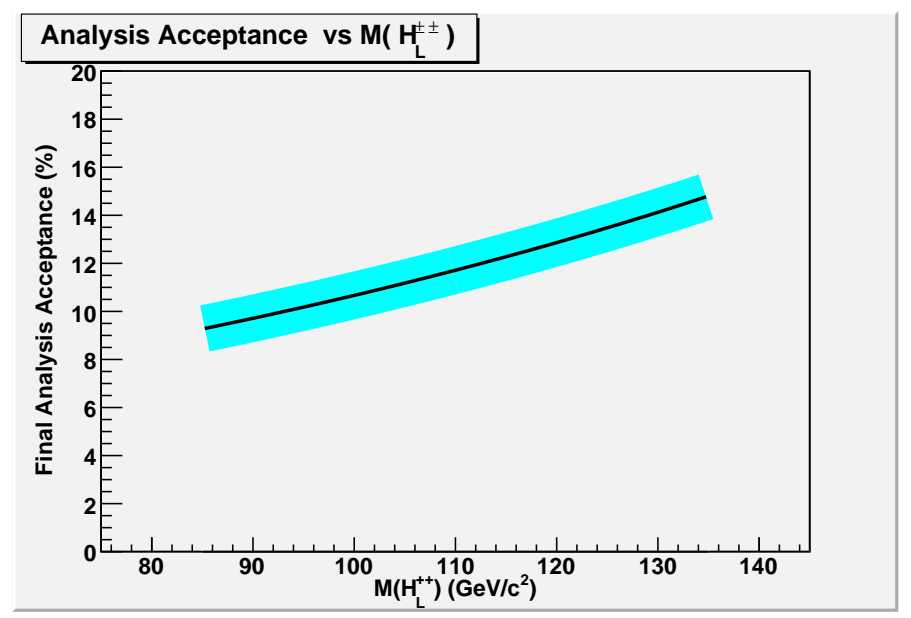

Figure 6.1: The combined acceptance, in percentage, for the three-lepton and fourlepton signatures, versus the mass of the $H^{ \pm \pm}$. The cyan band represents the combined statistical and systematical uncertainties associated with the signal acceptance.

\subsection{Systematic Uncertainties on Signal Acceptance}

The weighted systematic uncertainties used for the limit setting procedure are summarized in Table 6.3. We further discuss these uncertainties below. 


\subsubsection{Lepton Identification}

Lepton identification efficiencies are obtained from data samples whose purity is high and/or well-known. If the efficiencies in data and MC differ for a given selection, then a scale factor must be applied to the MC selection. This process is identical to the trigger simulation procedure described in 4.4.1.

Systematic uncertainties on the reconstruction and identification of electrons are taken from comparisons $\Upsilon \rightarrow e e$ and $Z \rightarrow e e$ events in MC and data[57, 58]. Systematic uncertainties on Tau identification are obtained from comparisons of $W\left(\rightarrow \tau \nu_{\tau}+0\right.$ jet $)$ events in MC and data[57, 59]. Systematic uncertainties on the reconstruction and identification of muons are taken from comparisons between $\mathrm{MC}$ and $Z \rightarrow \mu \mu$ data[52]. We note that these uncertainties are relatively small $(\sim 0.5 \%)$ because our lepton selections are not very stringent - in other words, it is easy for data and MC to agree with the relatively coarse resolution required by our lepton identification selections. For example, we do not apply the electron-rejection cut to our tau candidates. This cut relies on accurate simulation of both the hadronic and EM calorimeter responses to pions, and carries a $1.9 \%$ relative uncertainty. Systematic uncertainties on electron and muon identification are also small because we have no need to cut tightly on $E / P$ for electrons, and $\Delta x(s t u b, t r k)$ on muons. The systematic uncertainties on lepton identification are summarized in Table 6.3.

\subsubsection{Track Isolation}

We assign a $1.5 \%$ uncertainty on our knowledge of the track-isolation efficiency for any given lepton[59]. Because the underlying event (e.g. particles resulting from quark/gluon jets from ISR, or from secondary $p \bar{p}$ interactions) is the primary cause of track isolation inefficiency, the uncertainties on the leptons are highly correlated. We assume these effects are maximally correlated, and add the errors directly. An overall $4.5 \%$ uncertainty in the three-lepton channel, and a $6 \%$ uncertainty in the 4 lepton channel, result. These effects are identical for the $e \tau$ and $\mu \tau$ searches, and are 


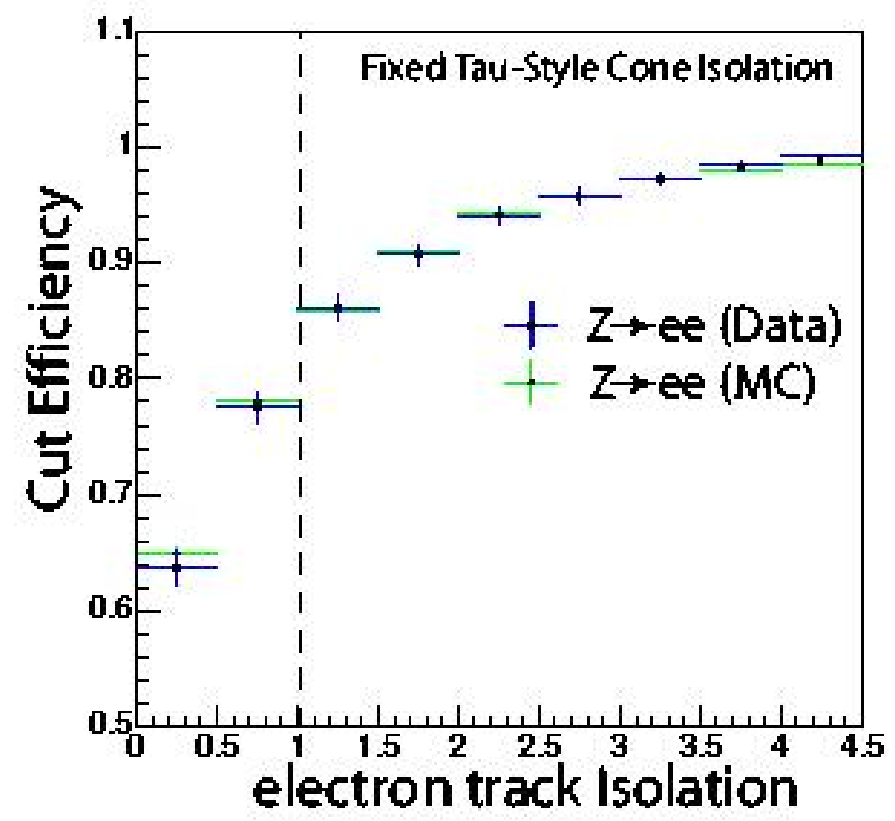

Figure 6.2: Tau-style track isolation cut efficiencies for $Z \rightarrow e e$ events in data and MC.

the dominant sources of systematic uncertainties associated with signal acceptance.

Figure 6.2 shows the Tau-Style TIso efficiency for electrons from $Z \rightarrow e e$ events $^{1}$. The two efficiencies seem to be in very good agreement, but the statistical error associated with the efficiency in data leads to the $1.5 \%$ systematic uncertainty.

\subsection{3 $\pi^{0}$ Isolation}

We assign a $0.5 \%$ uncertainty on our knowledge of the $\pi^{0}$-isolation efficiency for CdfTaus and ITSs[59]. Because the underlying event (e.g. particles resulting from quark/gluon jets from ISR, or from secondary $p \bar{p}$ interactions) is the primary cause of $\pi^{0}$-isolation inefficiency, the uncertainties on the leptons are correlated. Thus, we have an overall $1.0 \%$ uncertainty due to this effect in the three-lepton channel, and a $1.5 \%$ uncertainty in the 4-lepton channel. These effects are identical for the $e \tau$

\footnotetext{
${ }^{1}$ The main reason that leptons fail TIso is that a particle from the underlying event overlaps the given lepton. Such effects are identical for $Z \rightarrow e e, Z \rightarrow \mu \mu$, and $Z \rightarrow \tau \tau$ events, therefore the TIso efficiency as measured by $Z \rightarrow e e$ can apply to $Z \rightarrow \tau \tau$ events. The only tau-specific effect is that of a charged pion from tau-decay lying outside the tau signal cone $\alpha_{t r k}$ and causing the tau to fail TIso. This effect is small, and well-simulated by MC.
} 
and $\mu \tau$ searches.

\subsubsection{Calorimeter Energy Scale}

Our analysis is relatively insensitive to the EM or Hadronic calorimeter scale. We varied the scale of all measurements made by the hadronic calorimeter up and down by $6 \%$ and used the following convention to assign the systematic uncertainty associated with this effect:

- If both variations of the process in question (e.g. raising or lowering Had scale by $6 \%$ ) tend to increase(decrease) an analysis' acceptance, then the largest increase(decrease) in acceptance is taken as the systematic uncertainty on acceptance.

- If one variation of the process in question tends to increase acceptance, while another variation tends to decrease acceptance, the difference in the two varied acceptances is take as the systematic uncertainty on acceptance.

This change is $\sim 1 \%$ for the 3-lepton search, and $\sim 0 \%$ for the 4 -lepton search. The hadronic scale affects the tau track isolation because the cluster energy is used to define the tau's shrinking cone used for track isolation calculation. The hadronic scale can also affect muon ID efficiency. Apparently, both effects are very small. The same is true for the EM scale. We vary the EM scale up and down by $1 \%$. A maximum $1 \%$ change occurs in the three-lepton region, while the 4-track region seems to be unaffected.

\subsubsection{Parton Distribution Functions}

Uncertainties due to imperfect knowledge of the parton distribution functions (PDFs) were determined using the method [60] of weighting events according to their 'projections' on PDF parameters (eigenvectors), and noting the change in the overall weight as the eigenvectors are varied. The change in the Monte Carlo sample's weight (the sum of the weights of the individual events) that occurs with a standard 
variation of a given PDF eigenvector is the systematic uncertainty of the production cross section with respect to that eigenvector. The change in the weight of the subsample that is accepted by the analysis is the uncertainty in acceptance with respect to the varied PDF eigenvector. The uncertainty on acceptance influences the upper limit on the $\mathrm{H}^{++}$cross section, while uncertainty in cross section only affects the mass limit. In our case, the systematic uncertainty for acceptance due to PDFs is quite small (0.25\%). An explanation for this is that the kinematics of the final-state particles are dominated by the large masses of the $H^{ \pm \pm}$, and are thus insensitive to slight variations in the $H^{ \pm \pm} \mathrm{s} p_{T}$ and $\eta$.

\subsubsection{Initial State Radiation}

We first note that final-state radiation of quarks/gluons/photons by the $H^{ \pm \pm}$is not possible as the latter couple exclusively to leptons. Uncertainties due to variances in the amount of initial-state radiation are calculated by using the 'more/less' method [56]. The default ISR settings in PYTHIA are varied, and the corresponding changes in acceptances are taken as the systematic uncertainties due to ISR. After combining results from the three- and four-lepton searches, our analysis is relatively insensitive to such effects, due to compensatory changes in acceptance between the 3-lepton and 4-lepton channels. For example, the sample with increased ISR settings has a $2.6 \%$ decrease in 4-lepton acceptance, but a 3.8\% increase in 3-lepton acceptance, or a net $0.7 \%$ increase in acceptance. This effect can in part be explained by the reclassification of previously 4-lepton events as 3-lepton events. One of the four leptons may no longer have TIso $<2 \mathrm{GeV} / c$ due to a nearby quark or gluon jet that results from the increased ISR activity. However, the event will most likely pass all selections as a 3-lepton event. This process alone cannot contribute for the full $3.8 \%$ increase in the acceptance of the 3-lepton channel. The rest of the increase is presumably due to the increased energy-scale of the event (due to the presence of extra ISR activity), and, to a smaller extent, to an increase in the number of lepton candidates (perhaps 1\% of ISR jets may pass as hadronic taus). The overall 
effect on acceptance is below $1.5 \%$. We believe that the effect is even smaller, but were statistically limited by the sizes of our MC samples ${ }^{2}$. We do not attempt to reduce this uncertainty, however, as the uncertainty is small compared to that of track isolations $(5 \%)$.

\subsubsection{Trigger Efficiency}

A systematic uncertainty of $1.0 \%$ per leg is assigned due to trigger efficiency[52, 59].

\subsection{Systematic Uncertainties on Signal Production}

\subsubsection{Uncertainty on Theoretical Cross Section.}

A $7.5 \%$ uncertainty is used on the NLO cross section[61].

\subsubsection{Luminosity}

A $5.9 \%$ uncertainty is assigned to the luminosity, which has roughly equal components from the uncertainties on the CLC acceptance[53] and the total $p \bar{p}$ inelastic cross section[54].

\subsubsection{Parton Distribution Functions}

The systematic uncertainty associated with the production cross section is $5 \%$ for a Higgs mass of $110 \mathrm{GeV} / c^{2}$. This uncertainty is derived with the PDF variation method described in the previous section.

\footnotetext{
${ }^{2}$ All three samples (more/less/normal ISR), have equal acceptances within 1\%, but the statistical error associated with the samples is $1.5 \%$.
} 


\begin{tabular}{|c|c|c|c|}
\hline Effect & 3-Lepton & 4-Lepton & Combined \\
\hline \multicolumn{4}{|c|}{ Factors Associated with Acceptance } \\
\hline $\begin{array}{c}\text { PDF variation: Largest Increase } \\
\text { (Acceptance Only) }\end{array}$ & $+0.25 \%$ & $+0.25 \%$ & $+0.25 \%$ \\
\hline $\begin{array}{c}\text { PDF variation: Largest Decrease } \\
\text { (Acceptance Only) }\end{array}$ & $-0.25 \%$ & $-0.25 \%$ & $-0.25 \%$ \\
\hline $\begin{array}{l}\text { PDF variation: Net } \\
\text { (Acceptance Only) }\end{array}$ & - & - & $0.5 \%$ \\
\hline Trigger Efficiency & $1.4 \%$ & $1.4 \%$ & $1.4 \%$ \\
\hline ISR variation: More & $+3.8 \%$ & $-2.6 \%$ & $0.7 \%$ \\
\hline ISR variation: Less & $-2.4 \%$ & $+1.0 \%$ & $-0.6 \%$ \\
\hline ISR variation: Net & - & - & $1.3 \%$ \\
\hline Hadronic Scale: Up 6\% & $-0.25 \%$ & $-0.25 \%$ & $-0.25 \%$ \\
\hline Hadronic Scale: Down 6\% & $+1.0 \%$ & $+1.0 \%$ & $+1.0 \%$ \\
\hline Hadronic Scale: Net\% & - & - & $1.25 \%$ \\
\hline EM Scale: Up $1 \%$ & $+0.5 \%$ & $0.0 \%$ & $+0.2 \%$ \\
\hline EM Scale: Down 1\% & $-0.5 \%$ & $0.0 \%$ & $-0.2 \%$ \\
\hline EM Scale: Net & - & - & $0.4 \%$ \\
\hline Ele ID (Not TIso) & $0.5 \%$ & $0.5 \%$ & $0.5 \%$ \\
\hline Muon ID (Not TIso) & $.5 \%$ & $.5 \%$ & $.5 \%$ \\
\hline Tau ID (Not TIso) & $.8 \%$ & $.8 \%$ & $.8 \%$ \\
\hline 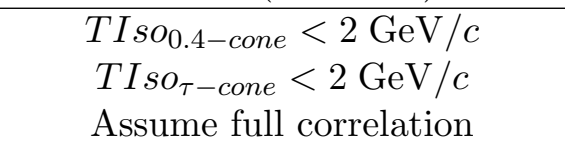 & $4.5 \%$ & $6.0 \%$ & $5.2 \%$ \\
\hline$\pi^{0} I s o<0.5 \mathrm{GeV}$ & $1.5 \%$ & $2.0 \%$ & $1.8 \%$ \\
\hline Sum & $8.17 \%$ & $7.61 \%$ & $6.03 \%$ \\
\hline \multicolumn{4}{|c|}{ Factors not associated with Acceptance } \\
\hline $\begin{array}{l}\text { PDF uncertainties } \\
\text { (Cross Section Only) }\end{array}$ & $5.0 \%$ & $5.0 \%$ & $5.0 \%$ \\
\hline Luminosity & $5.9 \%$ & $5.9 \%$ & $5.9 \%$ \\
\hline $\mathrm{NLO} \sigma$ & $7.5 \%$ & $7.5 \%$ & $7.5 \%$ \\
\hline Sum & $13.5 \%$ & 13.1 & $12.05 \%$ \\
\hline
\end{tabular}

Table 6.3: Systematics used for limit setting. The first and second columns show the weighted impact of a given uncertainty (i.e. $1.5 \%$ for track isolation applied 3 of 4 times per event). The last column shows the effect on the uncertainty if we take into account correlations between the 3 - and 4-lepton regions. 


\section{Chapter 7}

\section{Limit Setting Procedure}

The limit setting procedure[59] is finalized and validated before unblinding the analysis. As discussed earlier the background rates for the three-lepton and four-lepton regions are low. However, the probability to observe a single event is not negligible and given that the sensitivity improves if we treat the two regions separately, we choose to do so.

We build the likelihood function as follows: we start with full rates of signal and background processes, $\nu_{i}$ in each of the two regions $(i=1,2$ corresponding to the three- and four-lepton channels respectively) and use Poisson statistics to calculate the probability of the true rates being within $d \nu_{i}$ of $\nu_{i}$ given the number of observed events $N_{i}$ :

$$
d \mathcal{P}=\prod_{i=1,2} P\left(\nu_{i}, N_{i}\right) d \nu_{i}
$$

where $P\left(\nu_{i}, N_{i}\right)$ is a Poisson distribution for expected rate $\nu_{i}$ and the number of observed events $N_{i}$.

Each rate is a sum of rates for signal and backgrounds (subscripts $s$ and $b$, respectively):

$$
\nu_{1}=\nu_{1}^{b}+\nu_{1}^{s}
$$




$$
\nu_{2}=\nu_{2}^{b}+\nu_{2}^{s}
$$

We then perform a transformation of variables $\left(\nu_{1}, \nu_{2}\right) \rightarrow\left(\nu_{1}^{s}, \nu_{2}^{s}\right)$. We include existing prior knowledge about the backgrounds assuming that those are nuisance parameters and will be integrated out later:

$$
\frac{d \mathcal{P}}{d \nu_{1}^{s} d \nu_{2}^{s}}=\prod_{i=1,2} \int_{\nu_{i}^{b}=0}^{\infty} d \nu_{i}^{b} \exp \left[-\frac{\left(\nu_{i}^{b}-\nu_{i 0}^{b}\right)^{2}}{2 \sigma_{i}^{b^{2}}}\right] P\left(\nu_{i}^{s}, N_{i}\right)
$$

Finally, we replace the signal rates in the three- and four-lepton regions with physically meaningful cross-sections using $\nu_{i}^{s}=\sigma_{i}\left(p \bar{p} \rightarrow H^{++} H^{--}\right) \times L \times \alpha_{i}$, where $\alpha_{i}$ is the full selection efficiency of signal events for region $i$ and $L$ is the integrated luminosity. We include prior knowledge about the acceptance and its uncertainty, account that $\sigma=\sigma_{1}=\sigma_{2}$ (it is the same process) and re-write the probability function in its final form:

$$
\frac{d \mathcal{P}}{d \sigma}=\int \prod_{i=1,2} d \alpha_{i} \times \exp \left[-\frac{\alpha_{i}-\alpha_{i}^{0}}{2 \sigma_{\alpha i}^{2}}\right] \times L^{2} \times \times \alpha_{i} \times \frac{d \mathcal{P}}{d \nu_{1}^{s} d \nu_{2}^{s}} \delta\left(\sigma_{1}-\sigma_{2}\right)
$$

and define Likelihood as:

$$
\mathcal{L}(\sigma)=\frac{d \mathcal{P}}{d \sigma}
$$

We integrate over all integration variables in the physical region (all processes must have non-negative rates) to obtain the one-dimensional likelihood function as a function of the cross-section. We use $95 \%$ C.L. highest posterior density intervals to determine the maximum allowed cross-section value that we report as the final limit.

The procedure above does not include systematic uncertainties. To account for systematic effects $100 \%$ correlated between channels, we adjust $\alpha_{i}^{0}$ in the likelihood 


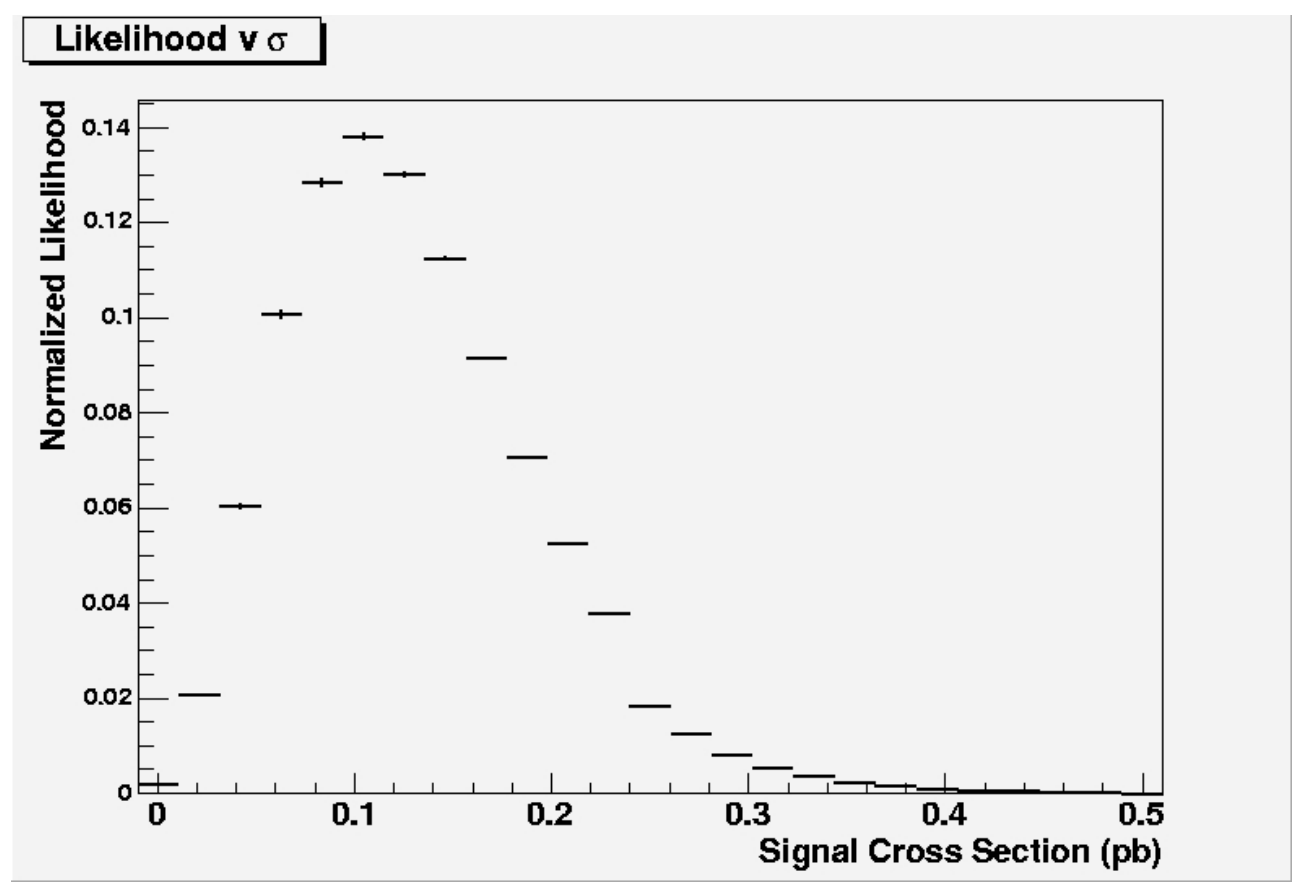

Figure 7.1: The likelihood outcome for a pseudoexperiment with a $100 \mathrm{fb} \mathrm{H}^{++}$signal.

to account for possible systematic shifts:

$$
\begin{aligned}
& \alpha_{1}^{0}=\alpha_{1}^{0} \times\left(1+\sum x_{k} \times \sigma_{k}^{1}\right) \\
& \alpha_{2}^{0}=\alpha_{2}^{0} \times\left(1+\sum x_{k} \times \sigma_{k}^{2}\right)
\end{aligned}
$$

where $k$ runs over all systematics types, $\sigma_{k}$ is the size of the systematic uncertainty of type $k$, and $x_{k}$ are corresponding eigenvectors. We then re-write Eq. 7.5 as

$$
\mathcal{L}(\sigma)=\int f(\vec{x}) d \vec{x} \mathcal{L}(\sigma, \vec{x})
$$

where $f(\vec{x})$ is the probability density function for $\vec{x}$ and is a product of individual PDFs (simple gaussian functions in our case). This procedure provides the desired values of correlations between the two channels. Figure 7.1 demonstrates the likelihood function for a pseudoexperiment with a signal of $100 \mathrm{fb}$. 


\subsection{Expected and Observed Cross section Limits}

Given the amount of expected backgrounds, there is a $75 \%$ probability of observing no events in either of the $e \tau$ signal regions, and a $63 \%$ probability of observing no events in either of the $\mu \tau$ regions. The expected cross section limit for both analyses is $\sim 95 \mathrm{fb}$ at $95 \%$ C.L., which translates to a Mass of $109 \mathrm{GeV} / c^{2}$ for the $H_{L}^{++}$. As final checks before looking in the signal region, we looked at the events that would have passed the $H_{T}$ cut had they not failed the $30<M(L S)<125$ cut. There were no events observed with like-sign mass higher than $125 \mathrm{GeV} / c^{2}$. Interestingly, there was one event in the $\mu \tau$ analysis that failed because the mass of the like-sign pair was too low. This event is discussed further in the appendix. There were no events observed in any of the signal regions of the two analyses. The limits obtained at $95 \%$ C.L. are $78 \mathrm{fb}$ for the $\mu \tau$ search and $74 \mathrm{fb}$ for the $e \tau$ search. These cross section limits translate to masses of $112 \mathrm{GeV} / c^{2}$ and $114 \mathrm{GeV} / c^{2}$ respectively for the $H_{L}^{++1}$. The mass limit for the $H_{R}^{++}$is $\sim 89 \mathrm{GeV} / c^{2}$, and does not surpass the current world limit, while that of the $H_{L}^{++}$does. This cross section limits, and the theoretical cross sections for the processes are plotted in Figure 7.2.

\subsection{Summary}

We have presented a search for the pair production of doubly charged Higgs' and subsequent lepton-flavor violating decays into muons and taus or into electrons and taus. There were no events observed in our $350 p b^{-1}$ and $322 p b^{-1}$ data samples, consistent with Standard Model expectations. We have set the world's highest mass-limits for the pair production of $H_{L}^{++} H_{L}^{--}$followed by exclusive decays into electron-tau or muon-tau pairs.

\footnotetext{
${ }^{1}$ We account for theoretical uncertainties on the signal cross section by including these in quadrature with the uncertainty on luminosity. Similar uncertainties on backgrounds are accounted for separately.
} 


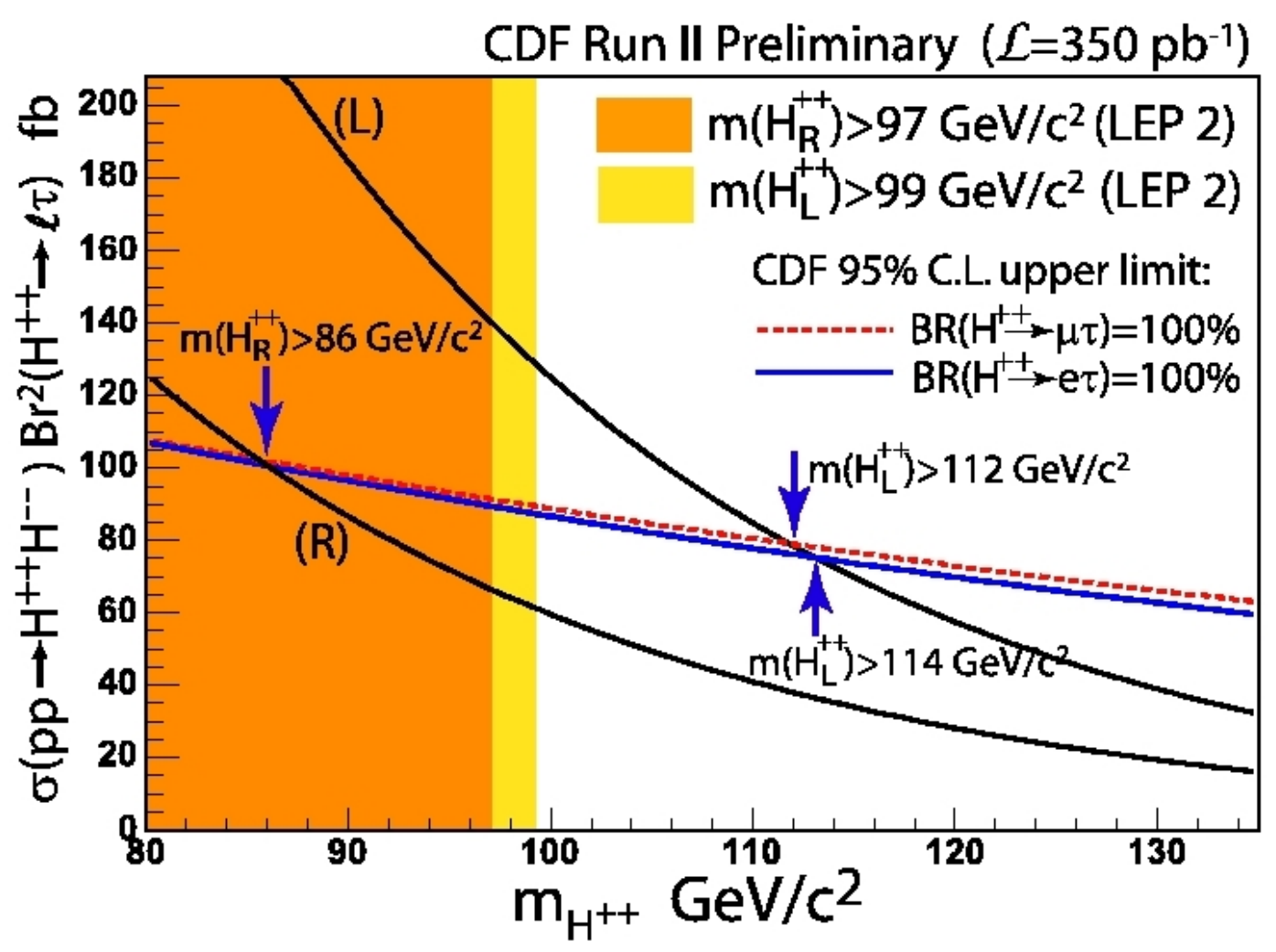

Figure 7.2: The cross section limits for $H^{++} H^{--} \rightarrow l^{+} \tau^{+} l^{-} \tau^{-}$at CDF. The CDF limit of $112 \mathrm{GeV} / c^{2}$ for exclusive $\mu \tau$ decays is shown by the dashed red line. The CDF limit of $114 \mathrm{GeV} / c^{2}$ for exclusive $e \tau$ decays is shown in the solid blue line. The LEP2 limits on the left and right-handed $\mathrm{H}^{++}$are shown by the shaded regions. 


\section{Appendix A}

\section{The Goldstone Theorem}

Here we present a short proof of the Goldstone Theorem[8].

Suppose we have a Lagrangian of $\mathrm{N}$ real scalar fields $\phi_{i}$ that are components of an N-dimensional vector $\Phi$,

$$
\mathcal{L}=\frac{1}{2} \partial_{\mu} \Phi \partial^{\mu} \Phi-V(\Phi)
$$

Let the Lagrangian be invariant under transformations of a continuous group $G$, and have $\Phi$ transform like

$$
\Phi \rightarrow \Phi^{\prime}=e^{-i \alpha^{a} T^{a}} \Phi .
$$

The transformations do not affect the potential:

$$
\delta V(\Phi)=\frac{\partial V(\Phi)}{\partial \phi_{i}} \delta \phi_{i}=-i \frac{\partial V(\Phi)}{\partial \phi_{i}} \alpha^{a}\left(T^{a}\right)_{i j} \phi_{j}=0
$$

This yields $\mathrm{N}$ equations

$$
\frac{\partial V(\Phi)}{\partial \phi_{i}}\left(T^{a}\right)_{i j} \phi_{j}=0
$$

One can take the derivative of the above equations:

$$
\frac{\partial^{2} V(\Phi)}{\partial \phi_{k} \partial \phi_{i}}\left(T^{a}\right)_{i j} \phi_{j}+\frac{\partial V(\Phi)}{\partial \phi_{i}}\left(T^{a}\right)_{i k}=0
$$


The second term is zero at the minimum of the potential, while the first term is contains the mass matrix:

$$
\left.\frac{\partial^{2} V(\Phi)}{\partial \phi_{k} \partial \phi_{i}}\right|_{\Phi=\Phi_{0}} \equiv M_{k i}^{2}
$$

So we have $\mathrm{N}$ equations of the form

$$
M_{k i}^{2}\left(T^{a}\right)_{i j} \phi_{j}^{0}=0
$$

If, after choosing a vacuum, there remains an n-dimensional sub-group of $\mathrm{G}$ that remains a symmetry of the vacuum, then for each generator of $g$ we have ${ }^{1}$

$$
\left(T^{a}\right)_{i j} \phi_{j}^{0}=0 \text { for } a=1, \ldots, n
$$

However, for the remaining N-n broken generators, we have

$$
\left(T^{a}\right)_{i j} \phi_{j}^{0} \neq 0 \text { for } a=n+1, \ldots, N
$$

Thus there must be N-n zero eigenvalues for the mass-matrix, according to (A.7). These are the massless Nambu-Goldstone bosons. Note that, since each NambuGoldstone boson corresponds to a gauge symmetry of the Lagrangian, it is possible to "gauge away" the bosons by a transformation to the "unitary gauge"

$$
\mathcal{L} \rightarrow e^{-\sum_{a=1}^{N-n}\left(T^{a}\right) \phi_{a}^{0}} \mathcal{L}
$$

\footnotetext{
${ }^{1}$ That is, $\delta \phi=0$ under transformations $T^{a}$ of the subgroup.
} 


\section{Appendix B}

\section{Massive Neutrinos}

The weak interaction treats left- and right-handed fermions differently, and the $\mathrm{SM}$ is structured accordingly. The Dirac spinors are decomposed into left- and

right-handed components with the projection operators $\hat{L} / \hat{R}=\frac{1}{2}\left(1 \pm \gamma_{5}\right)$. The lefthanded components transform as $S U(2)$ doublets, while the $S U(2)$ transformations act trivially in the right-handed components. This is the description of fermionic fields presented in the first few chapters of the usual textbooks. A second interpretation, however, is apparent during model building. Here we begin with massless fermions that are inherently left- or right-handed. The Yukawa coupling with the Higgs field results in a Dirac mass:

$$
\mathcal{L}_{\text {Yukawa }}^{l}=-G_{l} \frac{v+H}{\sqrt{2}}\left[\bar{l}_{R}\left(\begin{array}{ll}
0 & 1
\end{array}\right)\left(\begin{array}{c}
\nu_{L} \\
l_{L}
\end{array}\right)+\left(\bar{\nu}_{L} \bar{l}_{L}\right)\left(\begin{array}{l}
0 \\
1
\end{array}\right) l_{R}\right]
$$

We see that the Dirac mass is $-\frac{G_{l} v}{\sqrt{2}}$, and that the mass terms necessarily couple the left- and right-handed fields. My personal intuition sides with the decomposition of the massive Dirac field into left- and right-handed components, but the two interpretations are equivalent if one accepts the Higgs mechanism. In the SM, neutrinos are all left-handed and they cannot have a Dirac mass term like above. Perhaps the simplest way for the SM to accommodate neutrinos is to add a right-handed neu- 
trino field. There will be more parameters needed to describe the neutrino masses ${ }^{1}$, and no explanation of why the neutrino masses are so small - we will have fermion masses ranging from around 0.1 to $10^{11} \mathrm{eV}^{2}$.

There is another way to have massive neutrinos. The neutrino can be a Majorana particle $^{3}$. A Higgs triplet is necessary to give masses to Majorana neutrinos[62], and it is possible to construct a mass for the left-handed neutrino with or without the existence of right-handed neutrinos. If the neutrino is massive, then one may "boost" to a frame where a left-handed neutrino becomes right-handed. For Majorana neutrinos, one observer may boost along past a left-handed neutrino, turn around, and see a right-handed antineutrino. This implies lepton-number violation $\Delta l= \pm 2$, and leads to new physics. One such manifestation would be in neutrinoless double-beta decays: a neutron emits an electron and Majorana antineutrino which is absorbed by a nearby neutron (as a neutrino), inducing a decay to a proton and electron. It is possible to have double beta decay in the SM, but the final state contains two electrons and two neutrinos. The energy spectrum of the electrons will be very peaked only in the case of neutrinoless double beta decay. There are a handful of experiments that are currently searching for evidence of such decays. Observation of this process would favor models with Majorana neutrinos and Higgs triplets.

If there are right-handed neutrinos and Higgs triplets in addition to the SM, then it is possible to have Dirac and Majorana mass terms for the neutrinos. This results in the Seesaw mechanism which contains a "natural" link between parity violation and light neutrino masses.

\footnotetext{
${ }^{1}$ And even more to parameterize the mixing between the generations, which becomes possible (as in the quark sector) when the neutrino mass is not degenerate.

${ }^{2}$ Well, the fermion masses already span 5 orders of magnitude, but an extra 7 orders? There goes the neighborhood.

${ }^{3} \mathrm{~A}$ Majorana particle is its own antiparticle: $\nu_{M}=\frac{1}{2}\left(\psi+\psi^{c}\right)$, where $\psi^{c} \equiv \mathcal{C} \psi \mathcal{C}^{-} 1=i \gamma^{2} \psi^{*}$, with $\mathcal{C}$ being the charge conjugation operator.
} 


\section{Appendix C}

\section{Seesaw Mechanism}

If there are right-handed neutrinos, then one can make a Dirac mass for the neutrino:

$$
-m_{D}\left(\overline{\nu_{L}} \nu_{R}+\overline{\nu_{R}} \nu_{L}\right)
$$

It is possible to have massive Majorana neutrinos, without the requirement of right-handed neutrinos. The mass term would look like:

$$
-\frac{1}{2} m_{M}\left[\overline{\nu_{L}}\left(\nu_{L}\right)^{c}+\left(\overline{\nu_{L}}\right)^{c}\left(\nu_{L}\right)\right]
$$

where the superscript ${ }^{c}$ represents charge conjugation, $\mathcal{C} \nu \mathcal{C}^{-1}$, with $\mathcal{C} \equiv i \gamma^{1} \gamma^{2}$ as the charge conjugation operator . If there are right-handed neutrinos, then they would also have a mass with terms like (C.2). If both left and right-handed neutrinos exist, then, in principle, there could be mass terms of both the Dirac and Majorana types. We would have a mass matrix of the form:

$$
-\frac{1}{2}\left(\begin{array}{ll}
\overline{\nu_{L}^{c}} & \overline{\nu_{R}}
\end{array}\right)\left(\begin{array}{cc}
m_{L} & m_{D} \\
m_{D} & m_{R}
\end{array}\right)\left(\begin{array}{c}
\nu_{L} \\
\nu_{R}^{c}
\end{array}\right)+\text { h.c. },
$$

where $m_{D}$ is the Dirac mass, $m_{L}$ and $m_{R}$ are the left- and right-handed Majorana 
masses. The matrix in (C.3) has eigenvalues

$$
m_{ \pm}=\frac{1}{2}\left(m_{L}+m_{R} \pm \sqrt{\left(m_{L}-m_{R}\right)^{2}+4 m_{D}^{2}}\right)
$$

Where $m_{+}$and $m_{-}$are the masses of the heavier (mostly right-handed) and lighter (mostly left-handed) neutrinos. Now, we assume that $m_{R}$ is of the same scale as the VEV of the right-handed Higgs triplet in the left-right symmetric model, which is of the same scale as the right-handed $W, W_{R}$. We let the Majorana mass term for the left-handed neutrinos vanish, and we assume the Dirac masses for the neutrinos are identical to those of the corresponding charged leptons. This results in the masses:

$$
m_{+} \simeq m_{R} ; m_{-} \simeq \frac{m_{D}^{2}}{m_{R}} \simeq \frac{m_{D}^{2}}{m_{W}}
$$

So, the mass of the heavier, mostly right-handed neutrino is very large, while the mass of the mostly left-handed neutrino is very small. This is the seesaw mechanism - the small mass of the ordinary neutrino is related to the suppression of the righthanded weak current. If we use the WMAP[63] limits on neutrino masses $(\mathcal{O}(0.1)$ $\mathrm{eV} / \mathrm{c}^{2}$ ), then for the case of the tau lepton, (C.4) results in GUT-scale masses for the right-handed neutrinos. 


\section{Appendix D}

\section{Tau Energy Correction}

The default energy measurement for a hadronic tau candidate is given by the vector sum of the momenta of the charged and neutral decay daughters (pions)

$$
p^{\tau} \equiv \sum_{\Delta \Theta<\alpha_{t r k}} p^{\pi^{ \pm}}+\sum_{\Delta \Theta<\alpha_{\pi^{0}}} p^{0}
$$

where the energies of the neutral pions are given by:

$$
E^{\pi^{0}}=E_{\text {tower }}^{E M}-\sum_{\text {trk }}\left(0.3 \mathrm{GeV}+0.21 c \times p_{\text {trk }}\right)
$$

, and where there is an implicit assumption that the particles have masses of $140 \mathrm{MeV} / c^{2}$, as described in section 4.1.3. We call the 4 -vector in (D.1) the visible momentum, $p_{v i s}$, whose time-like component is $E_{v i s}$. The determination of the tau's visible momentum in (D.1) relies on efficient and accurate reconstruction of

neutral pions. Sometimes $\pi^{0} \mathrm{~s}$ are not well-reconstructed or not reconstructed at all. For example, a charged pion may pass within $3 \mathrm{~cm}$ of the neutral pion's cluster in the CSM and cause the $\pi^{0}$-reconstruction algorithm to be remove the $\pi^{0}$ from consideration. This process occurs more frequently in the high $p_{T}$ regime where the taus are highly boosted and collimated in the lab frame. Malfunctioning or broken wires/strips in the CSM are another cause of $\pi^{0}$-reconstruction inefficiency. 
Both scenarios can lead to mismeasurements of taus' energies, yet in both cases, the energy of the $\pi^{0}$ is usually deposited in the CEM even if a CSM cluster is not reconstructed or assigned to a $\pi^{0}$. In these cases, it may be possible to recover the energy of the "lost" $\pi^{0}$.

It is also possible to overestimate the tau's energy with (D.1). For example, if a charged pion leaves substantial energy in the CEM, perhaps due to charge exchange $\left(\pi^{+}+N \rightarrow \pi^{0}+P, \pi^{-}+P \rightarrow \pi^{0}+N\right.$, with the $\pi^{0}$ causing an electromagnetic shower in the CEM), then the formula (D.2) may overestimate the $\pi^{0}$ energy because it does not subtract the full EM energy deposition of the charged pion(s). The extreme example is in the (rare) case of an electron being misidentified as a hadronic tau. It may be possible to obtain an energy value of double the electron's energy by summing the energies of the electron's track and CEM/CSM cluster, which will be reconstructed as a $\pi^{0}$ as long as the track does not pass within $3 \mathrm{~cm}$ of the CSM cluster.

The misreconstruction of neutral pions is rare enough that the $E_{v i s}$ energy measurement is clearly superior to a pure calorimetry based energy measurement for taus. However, it is possible to improve the energy resolution by applying an algorithm that tries to identify cases where the $\pi^{0}$-energy is underestimated or overestimated. The algorithm starts by checking if the hadronic tau candidate is electronlike. If the fraction of the tau-cluster's total calorimeter energy that is just in the EM calorimeter (this is called the EM fraction, or Emfr) is high (above 90\%), then it is likely that a $\pi^{0}$ alone is not responsible for all the EM energy and that the $\pi^{0}$-energy will be overestimated. In these cases, the algorithm opts to choose the tau's calorimeter energy $\left(E_{C a l}\right)$ as the best energy measurement. In short, if the tau behaves like an electron, then energy-measurement that is appropriate for electrons is used. If the tau is not electron-like, then the algorithm creates a new energy measurement for the tau similar to $E_{v i s}$ :

$$
E_{\text {trks }+C E M}^{\tau} \equiv E_{M I P}^{\tau}=\sum_{\Delta \Theta<\alpha_{t r k}}\left(c \times p_{\text {trk }}^{\pi^{ \pm}}-0.3 \mathrm{GeV}\right)+\sum_{\text {Towers in Cluster }} E_{\text {Tower }}^{C E M}
$$


where $(-0.3 \mathrm{GeV})$ term accounts for the EM deposition of MIP-like charged pions, and is identical to the first term in (D.2). The quantity $E_{t r k s+C E M}$ given by equation (D.3), then has an inherent assumption that the charged pions are minimally ionizing in the CEM, therefore we can call it $E_{m i p}$. Two scenarios are likely if $E_{m i p}$ is substantially greater than $E_{v i s}$ :

- A: $\quad$ A $\pi^{0}$ has is misreconstructed or unreconstructed, and thus $E_{\text {vis }}$ underestimates that tau's energy.

- B: The charged pions are not MIPs in the CEM, and (D.3) overestimates the tau's energy.

In the first case, $E_{m i p}$ is assigned to the tau only if the algorithm can decide that the charged pions are indeed MIP-like in the EM calorimeter. The checks used here are:

1. $E_{m i p}$ and $E_{c a l}$ are more consistent with each other than are $E_{v i s}$ and $E_{c a l}$

2. The track-based and (hadronic-only)calorimeter-based energy measurement of the charged pions are roughly consistent: $\left(\left|E_{\text {trks }}^{\pi^{ \pm}}-E_{H A D}^{\pi^{ \pm}}\right| \leq 3 \sigma_{H A D}\right)$

In the second condition above, $\sigma_{H A D}$ is the $0.5 \times \sqrt{E}$ resolution of the hadronic calorimeter. If either of the checks proves false, then the likelihood of scenario $\mathbf{B}$ increases. In this case, we must avoid $E_{m i p}$, and choose between $E_{v i s}$ and $E_{c a l}$. If $E_{c a l}>E_{v i s}$, then it is still likely that a $\pi^{0}$ was misreconstructed. The algorithm first checks against gross overestimations by the Hadronic Calorimeter $\left(E_{H A D}-3 \sigma_{H A D}>\right.$ $\left.p_{t r k s}\right)$ then assigns $E_{c a l}$ as the tau's energy. Otherwise, the nominal $E_{t r k s+\pi^{0} s}$ is used. This algorithm is outlined in Figure D.1. In practice, the algorithm returns $E_{v i s}$ nearly $75 \%$ of the time, $E_{m i p} 15 \%$ of the time, and $E_{c a l} 10 \%$ of the time for hadronic taus. 


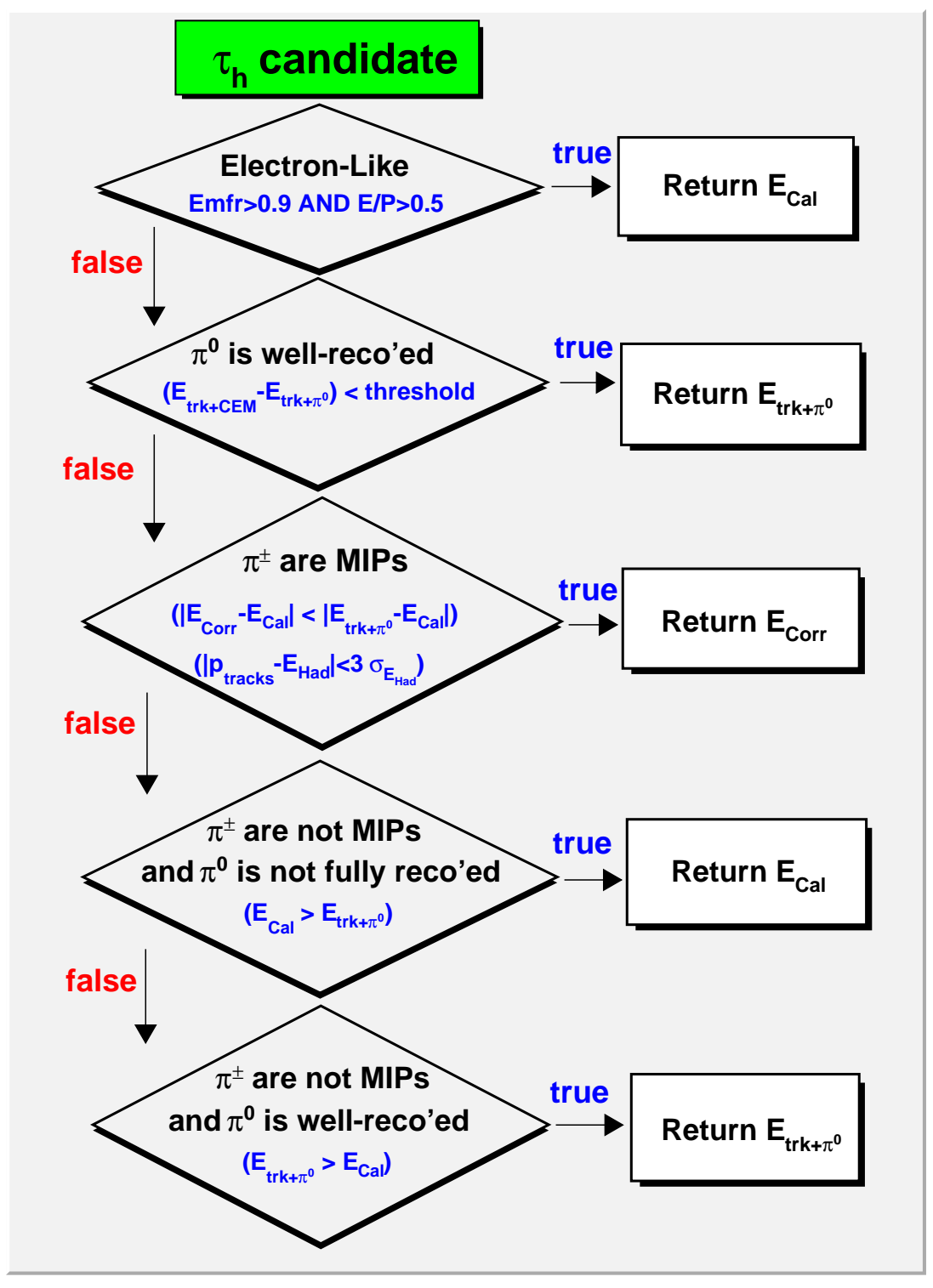

Figure D.1: A schematic representation of the tau energy-correction algorithm. 


\section{Appendix E}

\section{Conversion Tagging Efficiency}

It is possible to measure the conversion-tagging efficiency directly from data. We describe a method that relies solely on the silicon and drift trackers[55]. We do not reproduce the formal calculation described in [55] but rather give a conceptual description.

If a conversion is tagged by a conversion-tagging algorithm, then the radius of conversion is known. One can measure, for example, the hit efficiency for the first layer of silicon for all tracks that are tagged as conversions occurring at radii larger than the radius of the first layer. This efficiency should be zero except for accidental hit-matches, because photons do not leave tracks - for simplicity, let's take it to be identically zero. One can also measure the hit efficiency for a sample of tracks that are non-conversions, for example, tracks from identified muons, or from electrons from $Z \rightarrow e e$ events. For tracks that are extrapolated to pass through the fiducial region of the first silicon layer, this efficiency is pretty high - for simplicity, let's take it to be unity.

Suppose we have 1000 identified electrons that are extrapolated to pass through the fiducial region of the first layer of silicon, and we have tagged 80 of them as conversion-electrons. We would expect the first layer silicon hit efficiency of the 
entire sample, then, to be

$$
\epsilon_{s i}=\frac{1}{1000} \times[80 \times 0+920 \times 1]=0.92
$$

where $\epsilon_{s i}$ is the Layer- 1 silicon hit efficiency for the entire sample. If, for example, the conversion-tagging efficiency as actually 80\%, and there are actually 100 conversions in the sample, then we would measure a silicon hit efficiency of 0.9 for the 1000 electron sample.

$$
\epsilon_{s i}=\frac{1}{1000} \times[100 \times 0+900 \times 1]=0.90
$$

The deviation of the sample's hit efficiency from the efficiency measured by $Z \rightarrow \mu \mu$ or $Z \rightarrow e e$ events is related to the conversion content of the sample. The calculation used in [55] is slightly more complex because the silicon-hit efficiencies are not exactly zero and unity for conversion and prompt electrons respectively, and because information from all silicon layers, not just Layer 1, are used. 


\section{Appendix F}

\section{Interesting Event in Low $M_{L S}$}

\section{Region}

We observe one event with $H_{T}>190 \mathrm{GeV}$ in the 3-lepton $\mu \tau$ search. It fails only the $30<M_{L S}<125$ selection, with the invariant mass of the like-sign pair being 22 $\mathrm{GeV} / c^{2}$. There is little signal or background expected with such kinematics - the background levels for this region are $0.05+0.077 /-0.05$ events. This corresponds to a $5.6 \%$ probability of the backgrounds yielding 1 or more events in this region. However, if we were to drop the like-sign mass requirement, the expectation of observing one or more event with $H_{T}>190 \mathrm{GeV}$ rises to $30 \%$. We note that this event, due to the low invariant mass of the like-sign particles, is inconsistent with doubly charged higgs ${ }^{1}$. The event has a leading $100 \mathrm{GeV}$ muon, a $60 \mathrm{GeV}$ tau, and a $10 \mathrm{GeV}$ tau. The mass made by the leading muon and leading tau is $171 \mathrm{GeV} / c^{2}$. The mass made by the leading muon and second tau is $85 \mathrm{GeV} / c^{2}$. The mass made by the muon and $\mathbb{F}_{T}$ is $110 \mathrm{GeV} / c^{2}$. Table F.1 further describes the event.

The muon's high $p_{T}$ makes the event inconsistent with the $Z \rightarrow \tau_{\mu} \tau_{h}+$ jets process. Under this hypothesis (with the jet being the $60 \mathrm{GeV}$ tau, and the actual $\tau_{h}$ being the $10 \mathrm{GeV}$ tau), the mass of the $\mathrm{Z}$ would have to be at least $180 \mathrm{GeV}$.

\footnotetext{
${ }^{1}$ Unless, of course, one considers supersymmetric variants which involve cascade decays into taus, staus, and invisible LSP's.
} 


\begin{tabular}{|c|c|c|c|c|c|c|c|c|c|c|}
\hline \hline Object & $E_{T}$ & $p_{T}$ & $\eta$ & $\phi$ & $\mathrm{Q}$ & $\frac{\sigma_{\text {curv }}}{\text { curv }}$ & Si Hits & $N_{\text {Trks }}$ & Stubs & $N_{\pi^{0}}$ \\
\hline$\mu$ & 8 & 100 & .87 & 0.82 & -1 & 0.04 & $3_{A x} / 2_{S t}$ & 1 & CMX & 0 \\
\hline$\tau_{h}$ & 60 & 29 & -.13 & -2.67 & -1 & - & $6_{A x} / 5_{S t}$ & 1 & CMU & 2 \\
\hline$\tau_{h}$ & 10.6 & 6.2 & -.66 & -1.96 & -1 & - & $6_{A x} / 4_{S t}$ & 1 & - & 1 \\
\hline $\mathbb{E}_{T}$ & 34 & - & 0 & -1.63 & - & - & - & - & - & - \\
\hline
\end{tabular}

Table F.1: Characteristics of event 8996187, run 179055. The only reconstructed jets in the event are the two tau candidates. All three leptons come from the same vertex. There is an insignificant amount of energy associated with a second vertex.

The muon would carry roughly $80 \%$ of the tau's visible energy, and on the other side, the tau neutrino would carry over $80 \%$ of that tau's energy. A $Z \rightarrow \mu \mu+$ jet(s) event, with the softer muon being very soft, and possibly being reconstructed as one of the few tracks that have $p_{T}>500 \mathrm{MeV} / c$, would still be inconsistent with the substantial $\mathbb{F}_{T}$ present in the event. Our Monte Carlo samples predict $0.0_{-0.0}^{+0.06}$ $Z+$ jets events for the $Z \rightarrow \mu \mu$ and $Z \rightarrow \tau_{\mu} \tau_{h}$ channels combined. Dibosons predict $0.02 \pm 0.01$ events. Less significant backgrounds include $t \bar{t}$, cosmics, and QCD.

Of all SM backgrounds, $W+$ jets is the most significant in this region, with a prediction of $0.03+/-0.03$ events $^{2}$. The reconstructed mass of the muon and $\mathbb{F}_{T}$ is $110 \mathrm{GeV} / c^{2}$. It is possible that the energies of one or both tau objects are underestimated, causing extra $\mathbb{F}_{T}$, and a larger angle between the muon and the $\mathbb{F}_{T}$. This could explain the large $110 \mathrm{GeV} / c^{2}$ invariant mass made by the muon and $\not \mathbb{H}_{T}$.

An $r$ - $\phi$ display of the event is shown in Figure F.1. All tracks in the COT with $p_{T} \mathrm{~s}$ above $500 \mathrm{MeV} / c$ are shown. Figure F.2 displays all active calorimeter towers in the event with $|\eta|<3.0$ and $E_{T}>500 \mathrm{MeV}$.

\footnotetext{
${ }^{2}$ To achieve a low statistical error, we have combined the statistics of the $W\left(\rightarrow e \nu_{e}\right)$ and $W(\rightarrow$ $\left.\mu \nu_{\mu}\right)$ samples (treating the $e$ as a $\mu$ ) with a larger 6-million event $W\left(\rightarrow e \nu_{e}\right)$ sample after the completion of this analysis.
} 


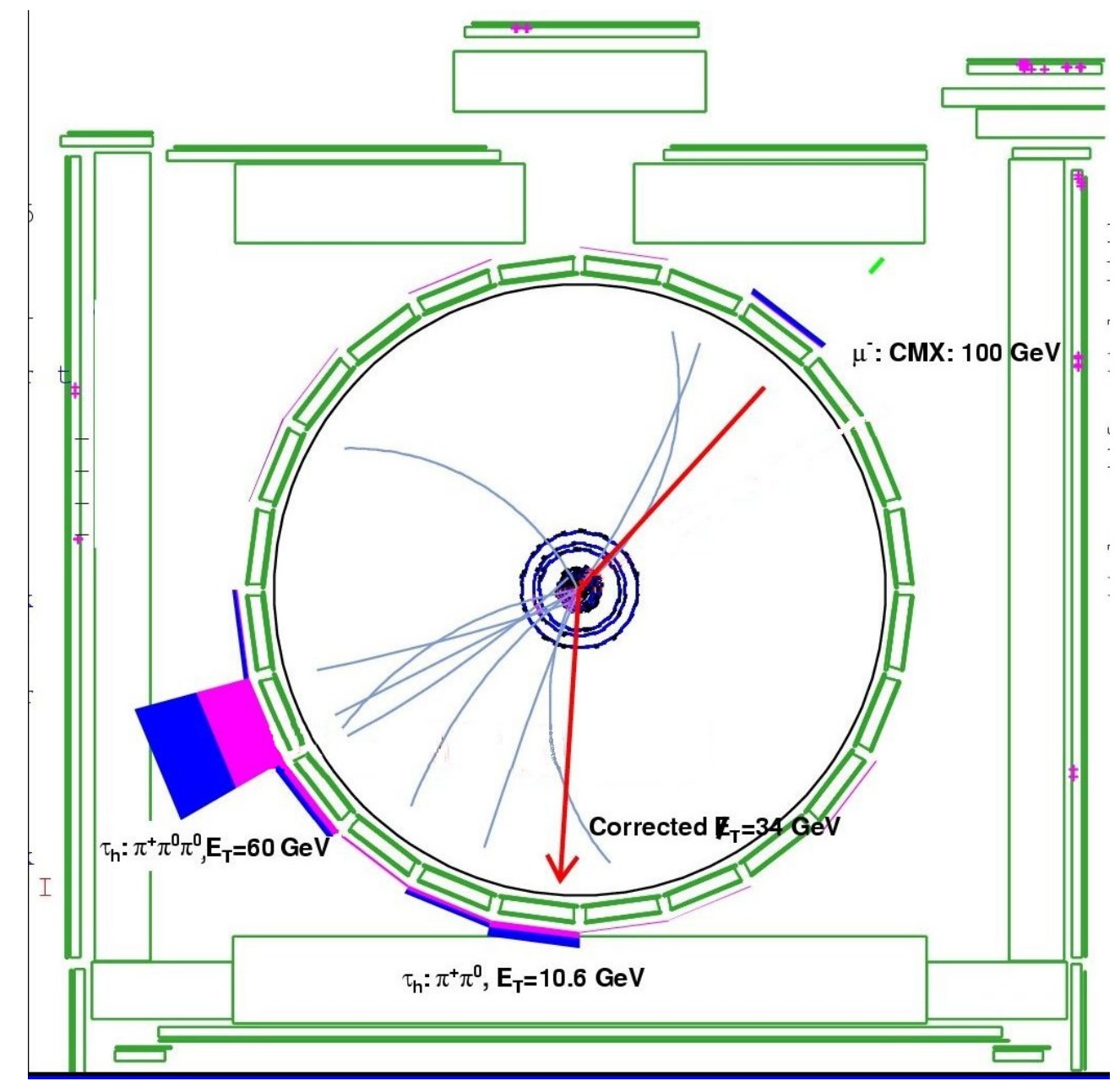

Figure F.1: The COT event display of the interesting event. All tracks with $p_{T}>$ $500 \mathrm{MeV} / c$ are shown. 


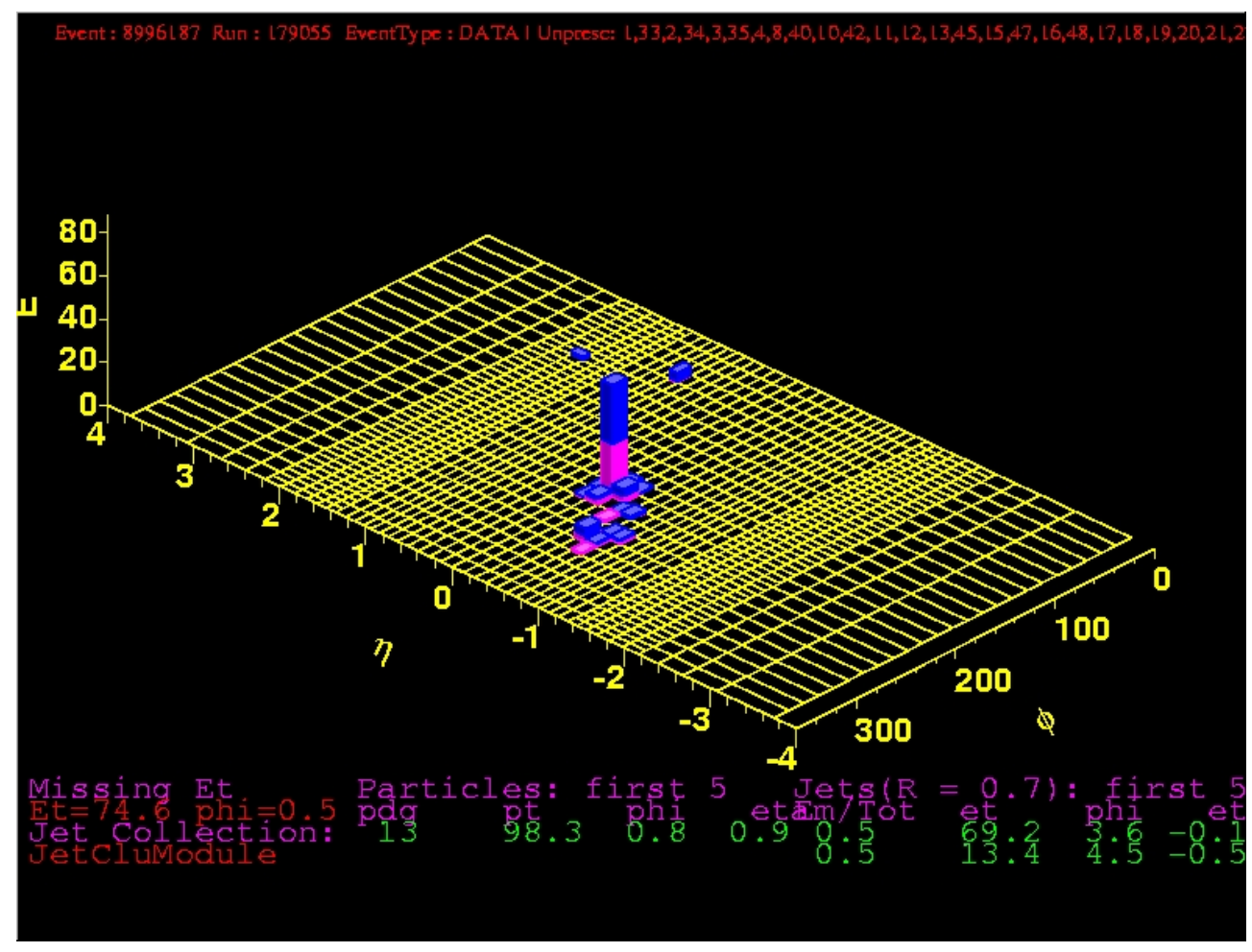

Figure F.2: The Calorimeter event display of the interesting event. All towers with $E_{T}>500 \mathrm{MeV}$ and $|\eta|<3.0$ are shown. 


\section{Bibliography}

[1] D. Griffiths, Introduction to Electrodynamics (Prentice Hall, Upper Saddle River, New Jersey, 1989).

[2] A translated version of Noether's Paper can be found at http://arxiv.org/pdf/physics/0503066.

[3] J. D. Jackson, Classical Electrodynamics (Wiley, New York, 1998).

[4] D. Griffiths, Introduction to Elementary Particles (Wiley, New York, 1987).

[5] M. E. Peskin and D. V. Schroeder, An Introduction to Quantum Field Theory (Perseus, New York, 1995).

[6] Q. Ho-Kim and X.-Y. Pham, Elementary Particles and Their Ineractions, Concepts and Phenomena (Springer, Berlin, 1998).

[7] M. Kaku, Quantum Field Theory, A Modern Introduction (Oxford University Press, New York, 1993).

[8] S. Novaes, Standard Model: An Introduction, (2000), particle and Fields, Proceedings of the X J. A. Swieca Summer School.

[9] J. C. Street and E. C. Stevenson, Phys. Rev. 52, 1003 (1937).

[10] T. D. Lee and C. N. Yang, Phys. Rev. 104, 254 (1956).

[11] C. Wu et al., Phys. Rev. 105, 1413 (1957). 
[12] M. Goldhaber et al., Phys. Rev. 1015 (1958).

[13] J. Schwinger, Ann. Phys 2, 407 (1957).

[14] T. Lee and C. Yang, Ann. Phys 2, 407 (1957).

[15] J. L. Lopes, Nucl. Phys 8, 234 (1958).

[16] S. L. Glashow, Nucl. Phys. 22, 579 (1961).

[17] G. t' Hooft and M. Veltman, Nucl. Phys. B44, 189 (1972).

[18] S. Eidelman et al., Phys. Lett B592, 1 (2004).

[19] R. S. Van Dyck, P. B. Schwinberg, and H. G. Dehmelt, Phys. Rev. Lett. 59, 26 (1987).

[20] A readable and illustrated description of the Fermilab Accelerator chain is available online at http://www-bd.fnal.gov/public/chain.html.

[21] The most recent version of the luminosity plot can be found at http://wwwbdnew.fnal.gov/operations/lum/lum.htm.

[22] D. Acosta, Physical Review Letters 95, 071801 (2005).

[23] A. Taffard et al., Run II Cosmic Ray Tagger, CDF Note 6100, (2003), http://www-cdf.fnal.gov/cdfnotes/cdf6100_CosTag_2.pdf.

[24] C. Currat et al., Single Pion Response in the CDF Calorimeters and Tuning GFLASH, CDF Note 5886, (2002), http://wwwcdf.fnal.gov/cdfnotes/cdf5886_GFLASH.ps.gz.

[25] A. Lobban and R. Wigmans, Intercalibration of the em and had sections of the Plug Upgrade Calorimeter: Part II, CDF Note 5586, (2001), http://wwwcdf.fnal.gov/cdfnotes/cdf5586_plug_intercal_2.ps.gz.

[26] A. Affolder et al., Fermilab-Pub 96/390-E, 1 (1996). 
[27] S. M. P. Skands and T. Sjostrand, Pythia 6.4 Physics and Manual, (2006), http://www.citebase.org/abstract?id=oai:arXiv.org:hep-ph/0603175.

[28] GEANT Detector Description and Simulation Tool, CERN Program Library Long Writeup W5013, (1994).

[29] A. G. Akeroyd and M. Aoki, Physical Review D 72, 035011 (2005).

[30] M. Muhlleitner and M. Spira, Physical Review D 68, 117701 (2003).

[31] B. Dutta and R. N. Mohapatra, Phys. Rev. D 59, 015018 (1998).

[32] The $H^{ \pm \pm} \rightarrow H^{ \pm} W^{ \pm}$and $H^{ \pm \pm} \rightarrow H^{ \pm} H^{ \pm}$decay modes do not become significant until $\mathrm{m}\left(H^{ \pm \pm}\right) \approx 160 \mathrm{GeV} / c^{2}$, as the $H^{ \pm}$mass below $80 \mathrm{GeV}$ is excluded at the $95 \%$ C .L by the DELPHI Collaboration, J. Abdallah and others., Eur. Phys. J. c34, 399 (2004); L3 Collaboration, P. Achard and others., Phys. Lett. B575, 208 (2003); ALEPH Collaboration, A. Heister and others., Phys. Rev Lett. B543, 1 (2002).

[33] E. Ma, M. Raidal, and U. Sarkar, Physical Review Letters 85, 3769 (2000).

[34] M. Kadastik, Doubly Charged Higgs Boson Decay to Muons at the LHC, (2005), www.nicpb.ee/ mario/msc.pdf.

[35] R. N. Mohapatra and G. Senjanovic, Phys. Rev. Lett. 44, 912 (1980).

[36] R. N. Mohapatra, Phys. Rev. D46, 2990 (1992).

[37] G. Abbiendi et al., Phys. Lett. B526, 221 (2002).

[38] J. Abdallah et al., Eur. Phys. J. C34, 399 (2004).

[39] P. Achard et al., Phys. Lett. B576, 18 (2003).

[40] A. Heister et al., Phys. Lett. B543, 1 (2002).

[41] V. M. Abazov et al., Phys. Rev. Lett. 93, 141801 (2004). 
[42] D. Acosta et al., Phys. Rev. Lett. 93, 221802 (2004).

[43] R. G. Wagner, Electron Identification for Run II: Algorithms, CDF Note 5456, (2003), http://www-cdf.fnal.gov/cdfnotes/cdf5456_electron_id_alg_v2_1.ps.gz.

[44] J. Marriner, Muon Upgrade Tube Drift Model, CDF Note 4816, (1992), http://www-cdf.fnal.gov/cdfnotes/cdf4816_Muon_Drift_Model.ps.gz.

[45] J. Bellinger et al., A Guide for Muon Reconstruction for Run 2, CDF Note 5870, (2002), http://www-cdf.fnal.gov/cdfnotes/cdf5870_muonreco.ps.gz.

[46] D. Acosta et al., Physical Review D 71, 112002 (2005).

[47] R. G. Wagner, Understanding and Using Lshr, CDF Note 5456, (2003), http://www-cdf.fnal.gov/cdfnotes/cdf6249_lshr_doc.ps.gz.

[48] S. Baroiant et al., Measurement of the Isolated Track Leg of the Electron+Track Trigger, CDF Note 6510, (2003), http://wwwcdf.fnal.gov/cdfnotes/cdf6510_tau_xft_efficiency.pdf.

[49] S. Baroiant et al., Lepton+Track Triggers: Measurement of the Level 3 Trigger Efficiency for Taus, CDF Note 6553, (2003), http://wwwcdf.fnal.gov/cdfnotes/cdf6553_lep+trk_L3_Tau_Effic.pdf.

[50] S. Baroiant et al., Measurement of Electron Trigger Efficienies for Level1 and Level2 8GeV Triggers, CDF Note 6257, (2003), http://wwwcdf.fnal.gov/cdfnotes/cdf6257_1112_eff_ele.ps.

[51] S. Baroiant et al., Measurement of the $8 \mathrm{GeV}$ Inclusive Electron Level 3 Trigger Efficiency Using Conversions (the CMUP case), CDF Note 6324, (2003), http://www-cdf.fnal.gov/cdfnotes/cdf6324_ele8GeV_L3efficiency.ps.

[52] V. Martin, High-Pt Muon ID Cuts and Efficiencies for use with 5.3.1 Data and 5.3.3 MC, (2005), http://wwwcdf.fnal.gov/cdfnotes/cdf7367_EffForTopExoticsGrl7.ps. 
[53] D. Acosta et al., A First Look at the CLC Luminosity Measurements, CDF Note 6052, (2003), http://wwwcdf.fnal.gov/cdfnotes/cdf6052_lum_run2_bless_v1.ps.gz.

[54] S. Klimenko, J. Konigsberg, and T. M. Liss, , fERMILAB-FN-0741.

[55] E. Halkiadakis et al., A Conversion Removal Algorithm for the 2003 Winter Conferences, CDF Note 6234, (2003), http://wwwcdf.fnal.gov/cdfnotes/cdf6250_conversion_opt.ps.gz.

[56] Y.-K. Kim and U.-K. Yang, Initial state gluon radiation studies on DrellYan data for top-pair production in hadron collider, CDF Note 6804, (2004), http://www-cdf.fnal.gov/cdfnotes/cdf6804_ISR_DY.ps.

[57] S. Baroiant et al., Measurement of the Tau ID Efficiencies for the First $72 \mathrm{pb}^{-1}$ of CDF Run II Data, CDF Note 7013, (2004), http://wwwcdf.fnal.gov/cdfnotes/cdf7013_tau_id_72.pdf.

[58] S. Baroiant et al., Electron ID Efficienies for Intermediate pT Using Upsilon to ee Events, CDF Note 7713, (2005), http://wwwcdf.fnal.gov/cdfnotes/cdf7713_Ele_ID_eff_at_mid_pT.ps.

[59] S. Baroiant et al., Measurement of the Z Production Crosssection in the Tau Channel, CDF Note 7589, (2005), http://wwwcdf.fnal.gov/cdfnotes/cdf7589_ztt_350.pdf.

[60] O. Gonzales and C. Rott, Uncertainties due to the PDFs for the gluino-sbottom search, CDF Note 7051, (2004), http://wwwcdf.fnal.gov/cdfnotes/cdf7051_pdfuncert_gluino.ps.

[61] M. Muhlleitner and M. Spira, Phys. Rev. D68, 117701 (2003).

[62] Y. Grossman, TASI 2002 lectures on neutrinos, (2003).

[63] A. Goobar, S. Hannestad, E. Mortsell, and H. Tu, JCAP 0606, 019 (2006). 